\title{
The Mis18 Complex: Integrating Temporal and Spatial Signals for Centromere Epigenetic Inheritance
}

\author{
Madison Elaine Stellfox \\ Pittsburgh, Pennsylvania
}

B.S. Biochemistry, Grove City College, 2008

A Dissertation presented to the Graduate Faculty

of the University of Virginia in Candidacy for the Degree of

Doctor of Philosophy

Department of Biochemistry and Molecular Genetics

University of Virginia

May, 2014 
(C) 2014 Madison Elaine Stellfox 


\section{Thanks and Dedications}

I would like to thank everyone who has helped this dissertation and degree become a reality, and I would like to dedicate my work to my family and friends in return for their constant love and support.

To my advisor, Dr. Daniel Foltz: thank you for all of the encouragement and direction you have given me throughout the last five years. I have received the best education possible because of you. To my committee members, Dr. P. Todd Stukenberg, Dr. Daniel Burke and Dr. M. Mitchell Smith: I thank you sincerely for the many hours of your expert and tireless mentorship. And to my labmates: without you my years in the lab would not have been nearly as successful or as fun.

To my incredible parents, Jonathan and Beverly Stellfox, and my awesome brother, Benjamin: you have been there for me from the beginning. From my first microscope to the final draft of this dissertation, you have been encouraging me every step of the way. To my extended family, in particular Donna and Sara Hunter; Alison, Andrea and Sadie Stellfox; my church family at Emsworth U.P. Church; the Wojtkowskis; and my two sisters (in the truest meaning of the word) Annale O'Donnell and Lindsay Stepanek: Life has been infinitely more joyous with all of you in it. And to my Charlottesville family, Mark, Joe and Judy Axelrod; Jaime Miller and Meghan Dailey: graduate school would have been utterly impossible without you. You have truly made Charlottesville my home away from home. And to my Lord and Savior: I have been through some very tough times and some very wonderful moments during these past six years, but you are always there to guide me, thank you. 


\section{Table of Contents}

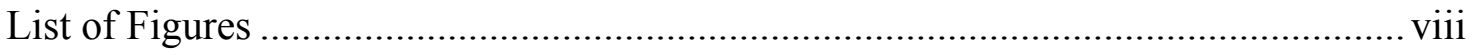

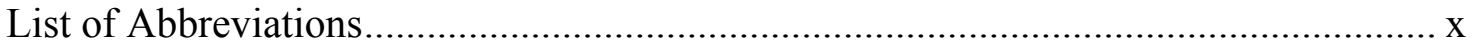

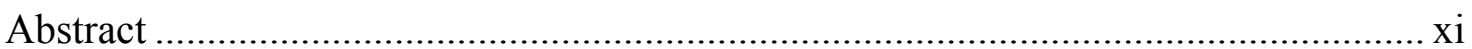

Chapter 1 - Centromere Structure, Function and Maintenance ..................................1

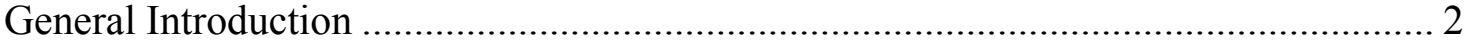

The Epigenetic Specification of the Centromere ................................................. 4

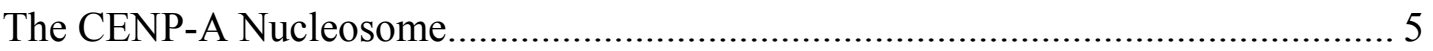

Centro-chromatin: Epigenetic context of CENP-A ............................................... 11

Influences of heterochromatin in centromere specification ................................. 16

The CCAN: Coupling Chromosomes to the Kinetochore............................................ 18

Maintaining Centromere Identity through DNA Replication ................................... 21

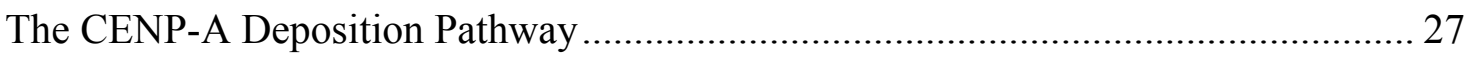

Initiation of CENP-A deposition: Key players ................................................... 27

Initiation of CENP-A deposition: Temporal regulation ...................................... 29

Initiating CENP-A deposition: Spatial regulation ............................................ 33

Priming the centromere for CENP-A deposition............................................ 35

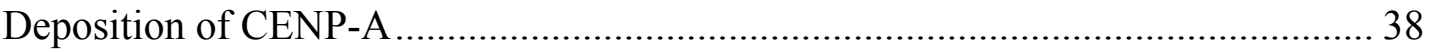

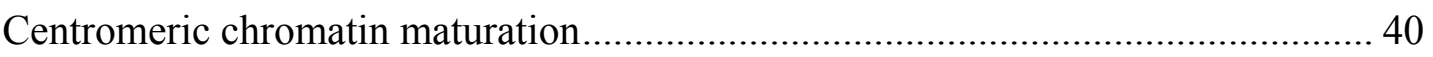

Non-coding RNAs, transcription and the centromere ........................................ 41

Ensuring CENP-A Centromere Specificity .......................................................... 44

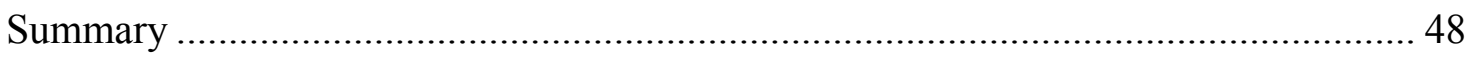

\section{Chapter 2 - The Mis18 Complex is Required for Cell Cycle Regulated CENP-A}

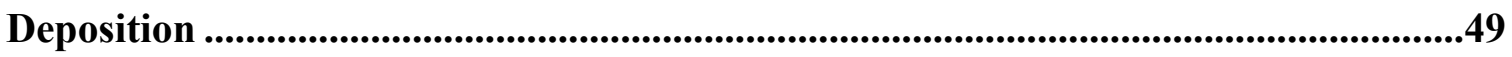

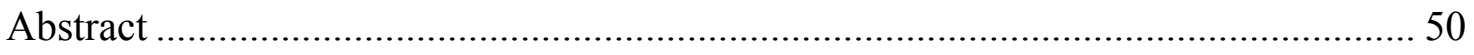

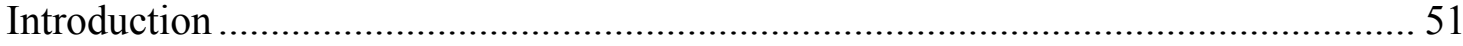

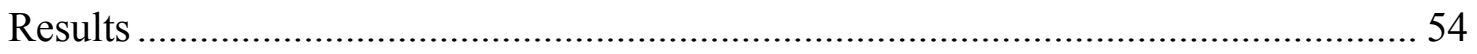

Recruitment of HJURP to centromeres requires the Mis18 Complex..................... 54

The Mis 18 complex regulates the cell cycle timing of CENP-A deposition.............. 58 
Mis18 $\alpha$ and Mis18BP1 bind centromeric chromatin with differing dynamics ......... 66

Mis18 $\alpha$ and Mis18 $\beta$ interact through conserved C-terminal coiled-coils ................. 71

Mis18 $\alpha$ and Mis18 $\beta$ recruit HJURP to centromeres for CENP-A deposition............ 76

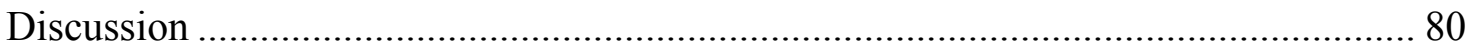

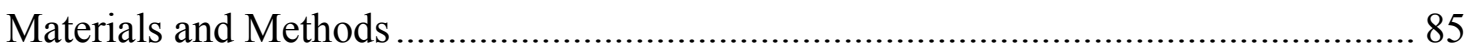

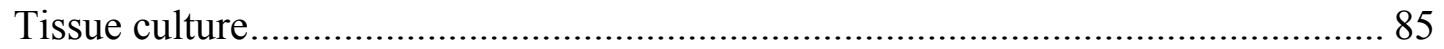

General immunofluorescence and image acquisition methods ............................. 85

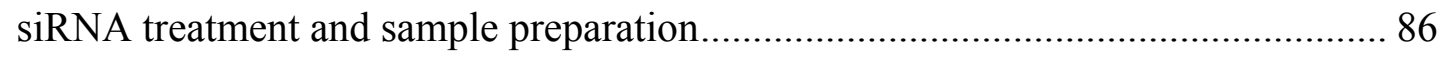

Fluorescent microscopy analysis of Mis18 and HJURP depletions ......................... 87

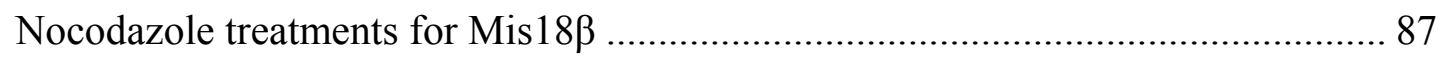

Mis18BP1 phospho-mutant transfection and western blot................................... 88

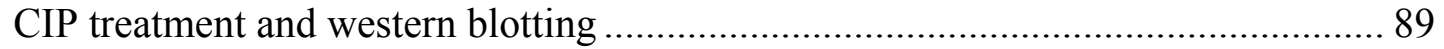

GFP-Mis18BP1 localization and CENP-A-SNAP tag experiments........................ 90

Fluorescence recovery after photobleaching (FRAP) ....................................... 90

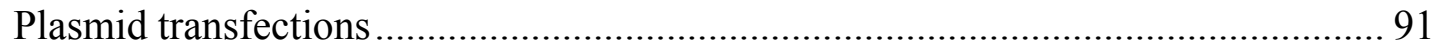

\section{Chapter 3 - Distinct Roles of Mis18 $\alpha$ and Mis18 $\beta$ in Cell Cycle Regulation of}

Centromere Specification ........................................................................................93

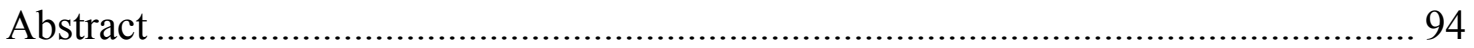

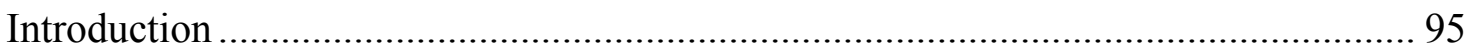

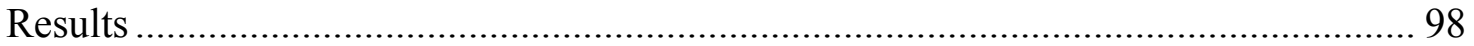

The N-terminus of Mis18BP1 is sufficient for centromeric localization and CENP-A

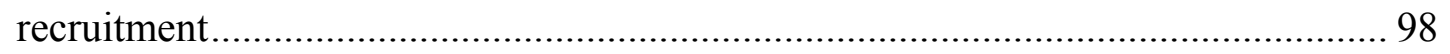

The extreme N-terminus of Mis18BP1 interacts with Mis18 $\alpha$........................... 103

Mis18BP1 exists in a separate complex from Mis18 $\alpha$ and Mis18 $\beta$ prior to G1 .... 107

Mis18 $\alpha$ interacts directly with Mis18BP1 through the Yippee/Mis18 domain....... 111

Mis18 $\beta$ binds CENP-C in a cell cycle dependent manner .................................... 115

The CENP-C-Mis18 $\beta$ interaction is regulated by kinase activity ......................... 120

Full recruitment of the Mis 18 complex by CENP-C requires multiple interactions 125

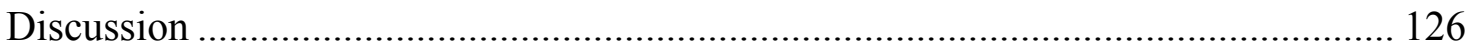

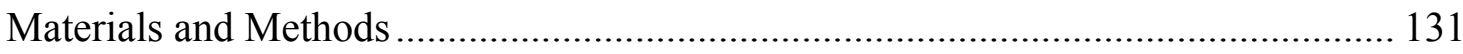


Indirect immunofluorescence and image acquisition 131

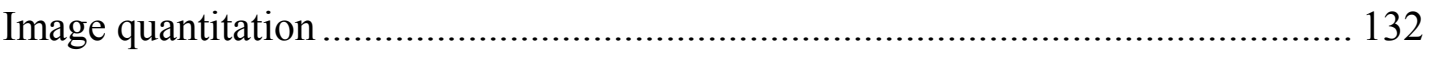

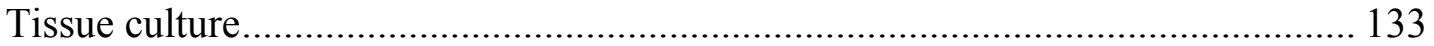

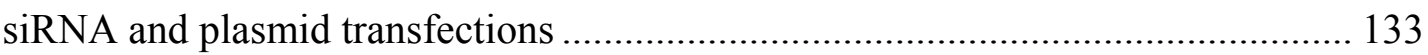

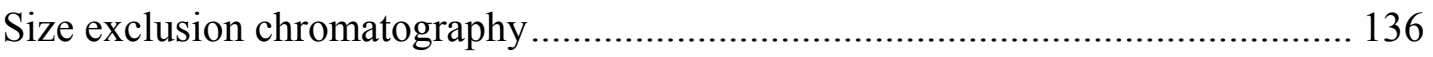

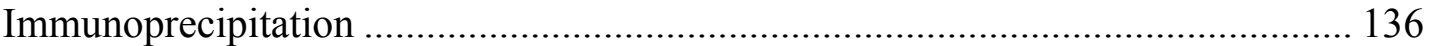

Recombinant proteins and in vitro pull-downs.............................................. 137

Nocodazole release and inhibitor treatments ................................................... 139

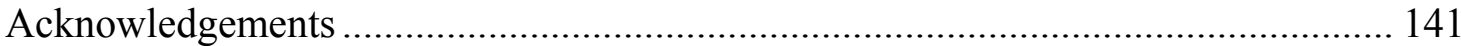

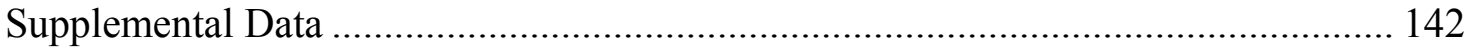

Chapter 4 - Preliminary Data and Future Directions .............................................147

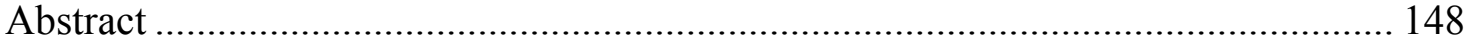

Mis18BP1-HP1 $\alpha$ interaction at pericentric heterochromatin ................................. 149

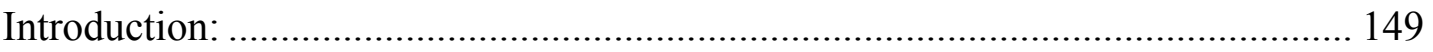

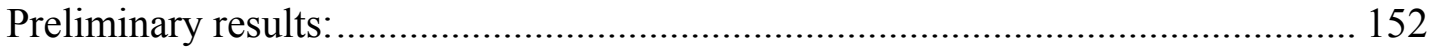

Mis18BP1 accumulates at pericentric heterochromatin in late G1 ................. 152

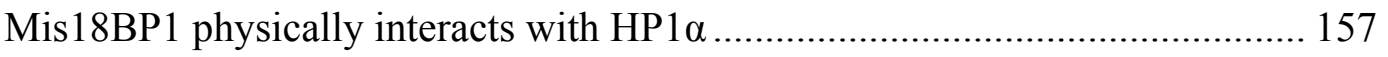

HP1 $\alpha$ is not required for centromeric recruitment of the Mis18 complex......... 162

Discussion and future directions: .................................................................... 165

Phospho-regulation of the Mis18BP1-HP1a interaction.................................. 165

Functions of the Mis18 complex outside of the CENP-A deposition pathway .. 168 Additional interactions between Mis18 and the CCAN........................................ 170

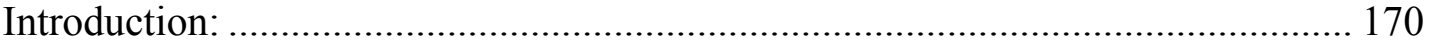

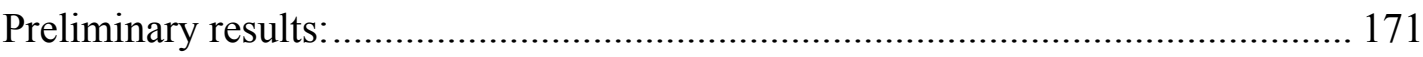

CENP-I and CENP-H recruitment effected by Mis18 $\alpha$-Mis18 $\beta$..................... 171

CENP-I and CENP-K depletions reduce Mis18 $\alpha$ localization in interphase...... 176

CENP-C is not required to maintain Mis $18 \alpha$ at the centromere ........................ 179

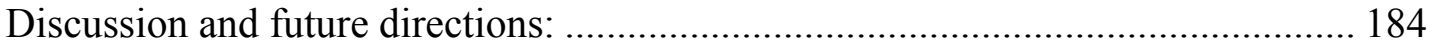

Possible histone binding capabilities of Mis18BP1 .............................................. 187

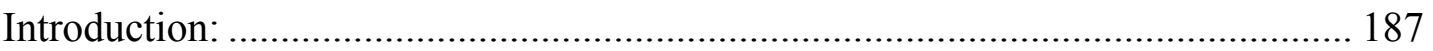




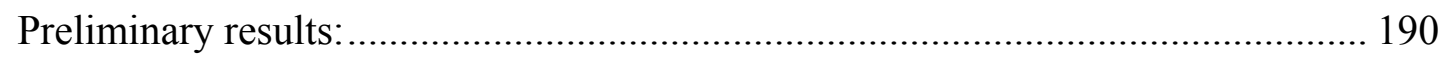

Mis18BP1 SANT domain is not sufficient for in vitro histone tail binding ....... 190

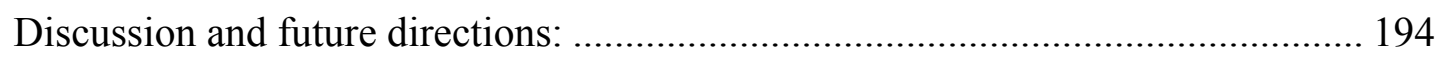

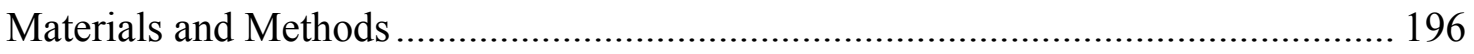

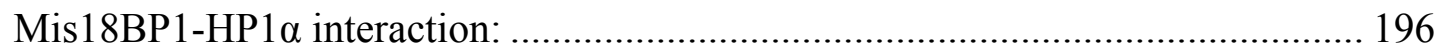

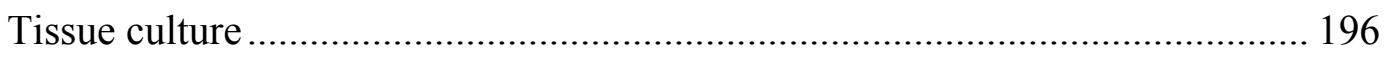

Immunofluorescence preparation and staining protocol.................................... 196

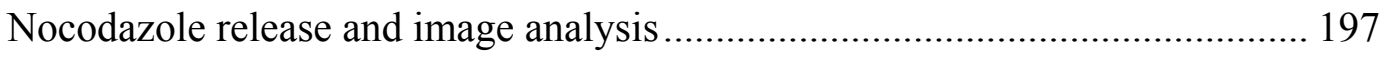

GFP-Mis 18BP1 fragment expression and localization analysis ......................... 198

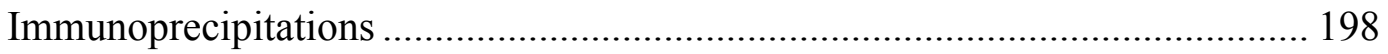

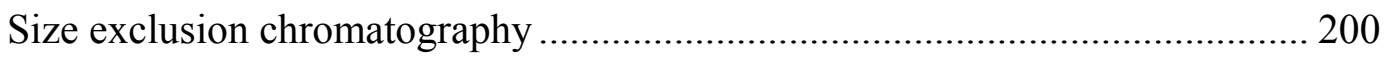

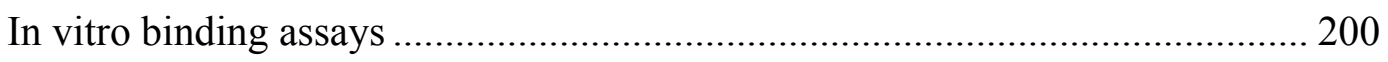

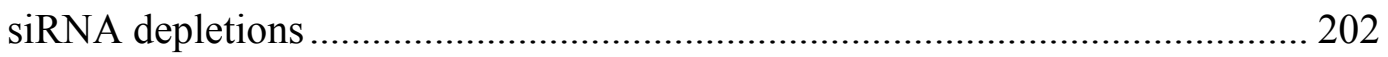

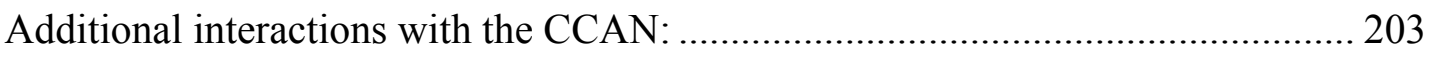

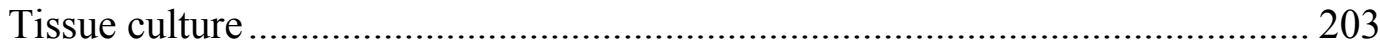

siRNA depletion and western blot ............................................................... 203

Immunofluorescence preparation and staining protocol.................................... 204

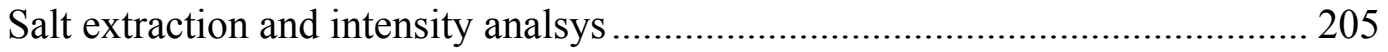

Possible histone binding capabilities of Mis18BP1: .............................................. 206

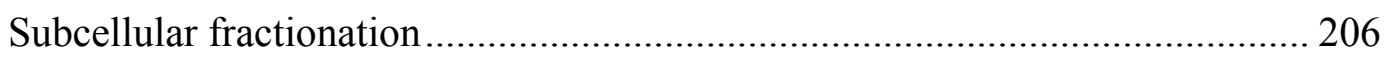

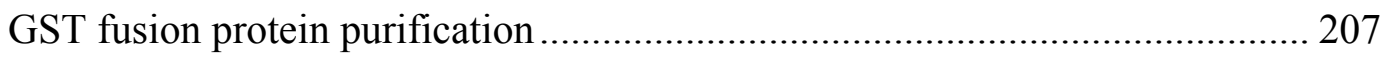

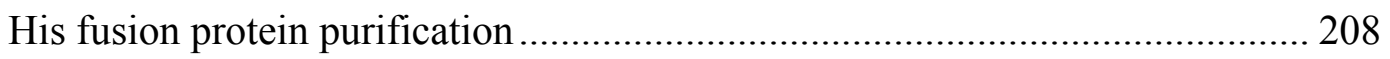

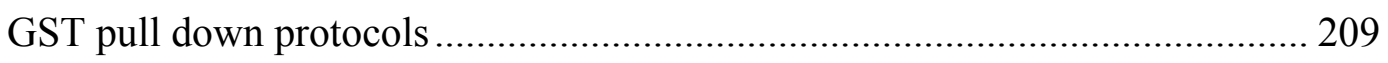

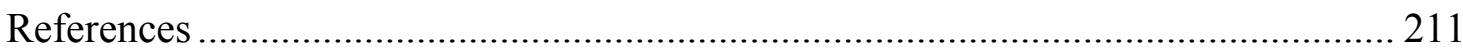

Appendix:.............................................................................................................229

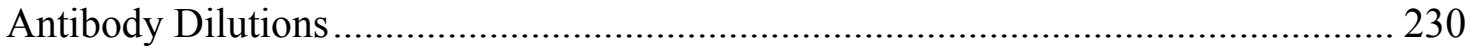

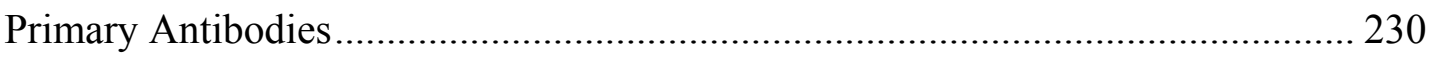

Secondary Antibodies.................................................................................... 230

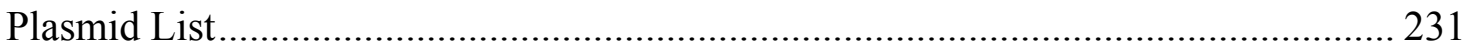




\section{List of Figures}

Figure 1-1. The CENP-A nucleosome: binding sites and structure..............................

Figure 1-2. The 3D organization of the human centromere .........................................14

Figure 1-3. Replication of centromeric chromatin and the $\mathrm{S}$ phase dynamics of the

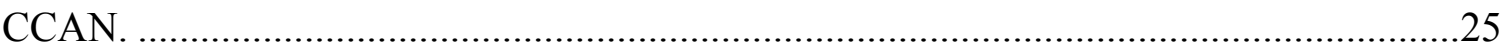

Figure 1-4. Overview of the cell cycle control mechanism of CENP-A deposition...........31

Figure 1-5. Removal of non-centromeric CENP-A through the proteasome pathway......46

Figure 2-1. Recruitment of HJURP to centromeres requires the Mis18 complex............56

Figure 2-2. The Mis 18 complex regulates the cell cycle timing of CENP-A deposition

Figure 2-3. Mis18 $\alpha$ and Mis18BP1 bind centromeric chromatin with differing dynamics.

Figure 2-4. Mis18 $\alpha$ and Mis18 $\beta$ interact through conserved C-terminal coiled-coils.......74

Figure 2-5. Mis18 $\alpha$ and Mis18 $\beta$ recruit HJURP to centromeres for CENP-A

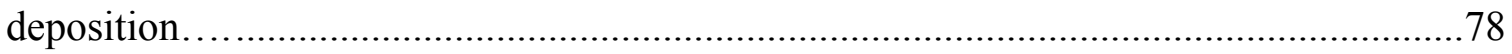

Figure 2-6. The deposition of newly synthesized CENP-A occurs during G1................83

Figure 3-1. Mis18BP1 N-terminus is required and sufficient for localization and function.

Figure 3-2. Mis18BP1 interacts with Mis18 $\alpha$ through its extreme N-terminus.

Figure 3-3. Mis18BP1 exists in a separate complex from Mis18 $\alpha$ and Mis18 $\beta$ prior to G1.

Figure 3-4. Mis18 $\alpha$ interacts with Mis18BP1 through conserved cysteine residues.......113

Figure 3-5. Mis18 $\beta$ binds CENP-C in a cell cycle dependent manner to generate full

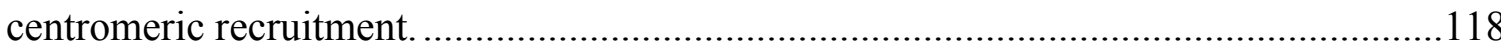

Figure 3-6. The CENP-C-Mis18 $\beta$ interaction is regulated by kinase activity.

Figure 3-7. The roles of the Mis18 $\alpha$ and Mis $18 \beta$ paralogs in centromere specification.

Supplemental Figure S3-1. Endogenous Mis18BP1 is not required for Mis18BP1 $1^{2-721}$ to localize to centromeres.

Supplemental Figure S3-2. Decrease in CENP-A levels validates Mis 18 complex depletion. 
Supplemental Figure S3-3. Full recruitment of the Mis 18 complex by CENP-C requires

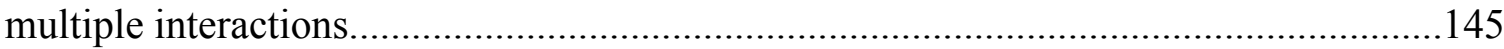

Figure 4-1. Mis18BP1 accumulates at pericentric heterochromatin in late G1..............155

Figure 4-2. Mis18BP1 physically interacts with HP1 1 . .................................................. 160

Figure 4-3. HP1 $\alpha$ is not required for centromeric recruitment of the Mis 18 complex....163

Figure 4-4. CENP-I and CENP-H recruitment effected by Mis18 $\alpha$-Mis $18 \beta$..................174

Figure 4-5. CENP-I and CENP-K depletions reduce Mis18 localization in

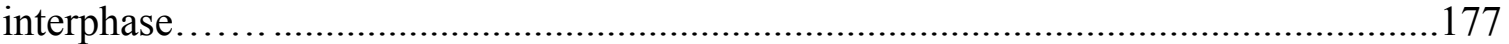

Figure 4-6. CENP-C is not required to maintain Mis18 $\alpha$ at the centromere. ..................182

Figure 4-7. Mis18BP1 contains a highly conserved SANT domain................................189

Figure 4-8. Mis18BP1 SANT domain is not sufficient for in vitro histone tail binding 


\section{List of Abbreviations}

\begin{tabular}{|c|c|}
\hline Abbreviation & Description \\
\hline a.a. & Amino acid \\
\hline Bub & Budding uninhibited by benzimidazoles \\
\hline CATD & CENP-A targeting domain \\
\hline CCAN & Constitutive centromere associated network \\
\hline Cdk & Cyclin dependent kinase \\
\hline CENP-A through -X & Centromere Protein -A through -X \\
\hline CID & Drosophila CENP-A homolog \\
\hline Cnp1 & S. pombe CENP-A homolog \\
\hline Cse4 & S. cerevisiae CENP-A homolog \\
\hline DAPI & 4,6-diamidino-2-phenylindole \\
\hline DMSO & Dimethyl sulfoxide \\
\hline DNA & Deoxyribonucleic acid \\
\hline GBP & GFP binding protein \\
\hline GFP & Green fluorescent protein \\
\hline HAC & Human artificial chromosome \\
\hline HJURP & Holliday junction recognition protein \\
\hline IF & Immunofluorescence \\
\hline IP & Immunoprecipitation \\
\hline KD & Kilodalton \\
\hline KNL & Kinetochore Null \\
\hline KNM & $\begin{array}{l}\text { Kinetochore complex: KNL-1, Mis } 12 \text { complex and the } \\
\text { Ndc } 80 \text { complex }\end{array}$ \\
\hline LacI & Lac repressor \\
\hline LacO & Lac operator \\
\hline MBP & Maltose binding protein \\
\hline Mis12, 16 and 18 & Missegregation of chromosomes phenotype mutants \\
\hline Mis18BP1/M18BP1 & Mis18 Binding Protein 1 \\
\hline Myr & Myristolated \\
\hline RbAp46/48 & Retinoblastoma associated protein $46 / 48$ \\
\hline RNA & \begin{tabular}{|l|} 
Ribonucleic acid \\
\end{tabular} \\
\hline SANT & Swi3-Ada2-NCor-TFIIIB domain \\
\hline SANTA & SANT-associated domain \\
\hline Scm & Suppressor of chromosome missegregation \\
\hline SDS-PAGE & Sodium dodecyl sulfate polyacrylamide gel electrophoresis \\
\hline ShRNA & Short hairpin ribonucleic acid \\
\hline SiRNA & Small interfering ribonucleic acid \\
\hline ZM447439 & Aurora B kinase inhibitor \\
\hline
\end{tabular}




\section{Abstract}

With every cell cycle, a cell must faithfully replicate its genetic information and distribute the duplicated genome equally between each daughter cell or risk genomic instability and cell death. To facilitate this essential process, centromeres are chromosomal domains that couple the pulling forces of the mitotic spindle to each sister chromatid. Centromere identity is dictated by the presence of nucleosomes containing the histone H3 variant, centromere protein A (CENP-A). Following S phase, newly synthesized nucleosomes must be deposited into chromatin in order to compensate for the dilution that occurs during chromatin replication. However, human cells do not deposit newly synthesized CENP-A nucleosomes until immediately after mitotic exit, in G1. While centromere architecture varies across species, the deposition of CENP-A is controlled by a group of conserved proteins, which includes the CENP-A assembly factor, Holliday junction recognition protein (HJURP) and the Mis 18 complex. The human Mis 18 complex consists of Mis18 $\alpha$, Mis $18 \beta$ and Mis18 binding protein 1 (Mis18BP1). In this dissertation, I will describe several aspects of this highly conserved complex and its role in the temporal and spatial regulation of CENP-A deposition. Chapter one will contain a general introduction to centromere architecture and function. In chapter two, I will describe how the Mis18 complex couples cell cycle progression to HJURP-mediated CENP-A deposition. In chapter three, I will detail the specific interactions that dictate Mis 18 complex formation and centromere recognition. Finally, chapter four will highlight new preliminary data that will drive future experimental explorations of additional mechanisms guiding Mis18 complex recruitment and function. 


\section{Chapter 1 - Centromere Structure, Function and Maintenance}

This chapter is based on the previously published manuscript:

Stellfox, ME, Bailey, AO, and Foltz, DR. Putting CENP-A in its place. Cellular and molecular life sciences: CMLS (2012). 


\section{General Introduction}

Accurate chromosome segregation is controlled by the centromere. The centromere is the chromosomal domain that directs kinetochore assembly thereby coupling microtubule pulling forces to each chromosome. In most eukaryotes, centromeres exist as a single locus on each chromosome, and a chromosome lacking a centromere will fail to segregate properly. Such segregation errors lead to aneuploidy, which in turn causes cellular stress and greater genomic instability [1]. On the other hand, a chromosome with too many centromeres leads to chromosome breakage, when an individual chromatid is attached to both spindle poles during mitosis and is torn apart. Such breakages can lead to breakage-fusion-bridge (BFB) cycles described originally by Barbara McClintock in 1941 [2]. BFB cycles may play a critical role in the creation of complex karyotypes often observed in cancers [3]. Therefore, a cell in these organisms must dedicate and maintain a single contiguous locus as the centromere among the millions of possible base pairs present on each chromosome. This process requires the involvement of a large, multi-protein centromere complex, which is directed by the cell cycle controlled assembly of centromere-specific nucleosomes, as well as chromatin remodeling and modifying activities, and the destabilization of centromere-specific nucleosomes at non-centromeric loci.

The evolutionarily conserved mark of centromere location is the presence of a unique nucleosome in which canonical histone $\mathrm{H} 3$ is replaced by CENP-A (Cse4 in budding yeast, Cnp1 in fission yeast, and CID in fruit flies). CENP-A is absolutely essential for viability in all organisms tested $[4,5]$. While the presence of a centromere- 
specific nucleosome is conserved throughout eukaryotic evolution, several features of the centromere including its organization, underlying DNA sequence, and mechanism of assembly are quite divergent. The budding yeast, $S$. cerevisiae, and a number of its relatives, determine the location of their centromeres through a specific DNA sequence to generate a so called "point centromere" consisting of 125 base pairs that position the centromere specific nucleosome [6, 7]. Fission yeast, S. pombe, and higher eukaryotes have more expansive genomic regions assigned as centromeres and employ epigenetic mechanisms to specify centromeric location at a single site on each chromosome. In contrast to both of these cases, the nematode, C. elegans, assembles centromeres along the entire length of its chromosomes instead of at a single site [8]. Despite these differences, all known centromeres depend on the presence of the centromere-specific nucleosome that contains the $\mathrm{H} 3$ variant, CENP-A. Only recently have we begun to appreciate the fact that despite vast differences in centromere architecture throughout evolution, many of the proteins and mechanisms involved in CENP-A deposition are highly conserved [9]. 


\section{The Epigenetic Specification of the Centromere}

The majority of higher eukaryotes with regional centromeres have unique DNA sequences that underlie endogenous centromeric chromatin. These loci tend to contain highly repetitive sequences. In humans and other mammals, the centromeric DNA is made up of tandemly arrayed alpha-satellite repeats [10]. However, the heritability of experimentally induced and naturally occurring neocentromeres and pseudodicentric chromosomes provides compelling evidence that the site of centromere formation and maintenance is epigenetically determined.

There are numerous examples in humans where an initially non-centromeric locus, outside of the endogenous alpha-satellite region, becomes an active centromere. These regions are called "neocentromeres" and they arise stochastically, at very low frequency, and without rearrangements of the underlying DNA [11]. Psuedodicentric chromosomes can occur through DNA translocations or inverted duplications and result in a single chromosome with two alpha-satellite containing regions. In psuedodicentrics, only one of the two alpha-satellite regions remains active, recruiting CENP-A nucleosomes, and the other loses its centromeric function.

Neocentromeres have been observed on almost every human chromosome but appear to cluster around certain regions within a given chromosome [11]. The nonrandom distribution of neocentromeres across the human genome suggests that not all sites have an equal potential to support centromere activity. This may be due either to unknown DNA sequences that are refractory to centromere formation or to chromatin states that may be more or less favorable for stable centromere formation. 
Neocentromeres and active centromeres of pseudodicentric chromosomes recruit CENP-A and all other centromere proteins that have been tested, except the DNA sequence-specific binding protein, CENP-B, which only localizes to DNA that contains CENP-B boxes [12-15]. The epigenetic inheritance of regional centromeres has been experimentally demonstrated in fission yeast and flies through the experimental generation of stable neocentromeres on chromosomal fragments that lack an original centromeric locus $[16,17]$. These data suggest that DNA sequence is neither necessary nor sufficient for centromere specification, and that the proteins associated with this region are the key determinants of centromere identity. Such plasticity in centromere location means that not only can CENP-A nucleosomes be deposited in a variety of chromosomal domains, but the machinery that deposits CENP-A nucleosomes into chromatin must also be able to function at these various sites.

\section{The CENP-A Nucleosome}

The CENP-A nucleosome is sufficient to specify the site of centromere formation and distinguish it as the location for kinetochore assembly during mitosis $[18,19]$. The centromere is occupied throughout the cell cycle by a large multi-subunit complex of proteins termed the CCAN (Constitutive Centromere Associated Network) comprised of 16 centromere proteins (CENPs C, H, I, K through U(50), W and X) [20-22]. It is the CCAN which mediates the assembly of the kinetochore in mitosis. The CCAN assembles only at centromeres and therefore distinguishes CENP-A nucleosomes from the H3containing nucleosomes found in general chromatin. 
The overall structure of the CENP-A nucleosome as well as particular sequences within have been proposed to be defining features that mediate the specificity of CCAN recruitment to CENP-A chromatin. Several provocative forms of the CENP-A nucleosome, other than the canonical octamer, have been proposed in an attempt to describe the uniqueness of the CENP-A nucleosome. The different proposed forms include a heterotetrameric form containing a single copy of each histone (CENP-A, histone $\mathrm{H} 4, \mathrm{H} 2 \mathrm{~A}$ and $\mathrm{H} 2 \mathrm{~B}$ ) in flies and humans, as well as a hexameric form in yeast that excludes $\mathrm{H} 2 \mathrm{~A}$ and $\mathrm{H} 2 \mathrm{~B}$ but contains the chaperone Scm3 [23-25].

Recently, the crystal structure of the human CENP-A nucleosome was solved, revealing an octameric nucleosome that wraps DNA in a left-handed manner similar to the H3-containing nucleosome [26]. Additional evidence for an octameric nucleosome structure comes from in-depth mutational studies of CENP-A. Mutations that disrupt the CENP-A-CENP-A interface in humans and flies preclude the stable incorporation of CENP-A into chromatin (Figure 1-1) [27, 28]. While these data do not rule out the possibility that CENP-A nucleosomes exist in multiple forms, it appears that the formation of an octameric structure is possible and important for initial stable CENP-A incorporation.

The overall protein structure of the CENP-A nucleosome is very similar to the histone H3-containing nucleosome. However, there are several features of the CENP-A nucleosome that distinguish it from canonical H3, which are highlighted in the schematic of human CENP-A in Figure 1-1A. The CENP-A targeting domain (CATD) consists of the unique residues within the loop 1 and alpha-2 helix of the CENP-A histone fold. The 
CATD is sufficient for centromere localization and confers a unique rigidity to the CENP-A nucleosome that may be a defining characteristic $[29,30]$.

Structural studies have identified two other regions of the human CENP-A nucleosome that stand out relative to canonical nucleosomes. The most $\mathrm{N}$-terminal helix of CENP-A, the alpha-N helix, contains three fewer residues and is therefore approximately one helical turn shorter than the comparable helix in histone H3 [26]. This region of the nucleosome is interesting as it is also the DNA entry/exit site. Consistent with a shorter alpha-N helix is the observation by several groups that CENP-A nucleosomes protect a smaller fragment of DNA in nuclease assays due to a partial unwrapping of the DNA at the entry/exit sites [26, 31, 32]. Correlative data from hydrogen-deuterium exchange mass spectrometry experiments, measuring protein dynamics, show that CENP-A exchanges protons 10 -fold faster than $\mathrm{H} 3$ at the $\alpha-\mathrm{N}$ helix even in extended nucleosome arrays [31].

The second important region of distinction is loop 1 of the human CENP-A histone fold, which was observed to form a surface-accessible bulge using Arg80/Gly81 in the CENP-A heterotetramer and in the CENP-A nucleosome [26, 33]. The extra two amino acids that form the bulge are a conserved feature of CENP-A homologs across all species (although $S$. pombe contains an even larger expansion); however, conservation of the positively charged arginine is restricted to mammals and birds. This bulge is accessible on the surface of the CENP-A nucleosome and could therefore serve as a recognition motif (Figure 1-1B). The surface bulge is not essential for centromeric targeting, but it is required for stable incorporation of the CENP-A nucleosome. 
Coexpression of wild type and CENP-A ${ }^{\Delta \mathrm{R} 80 \mathrm{G} 81}$ confirmed that the mutant protein initially localized to centromeres, but the number of cells with the mutant protein localized to centromeres decreased over several days [26].

Until recently phosphorylation of serine 7 was the only known posttranslational modification of CENP-A. CENP-A is phosphorylated at serine 7 by the Aurora A and Aurora B kinases beginning in prophase and throughout mitosis [34-36]. Phosphorylation at serine 7 was found to be essential for proper mitosis and cytokinesis [34-37]. Recently, CENP-A has been identified as a target of N-terminal RCC1 methyltransferase, NRMT [38-40]. The initiating methionine is cleaved from CENP-A, and the subsequent glycine residue is N-terminally trimethylated [40]. In addition, CENP-A also appears to be phosphorylated on serine 16 and serine 18 (S17 and S19 in Figure 1-1, which includes the initiating methionine) [40]. Similar to phosphorylation of serine 7, serine 16 and 18 phosphorylations appear to be required for proper mitosis and may play a role in ordered structure of the centromere [40]. While the specifics underlying the writing and downstream functions of these new posttranslational modifications have yet to be detailed, CENP-A specific posttranslational modifications indicate new possible mechanisms to organize centromeric structure and mediate centromere function. 
Figure 1-1. The CENP-A nucleosome: binding sites and structure.

(A) Primary sequences of human CENP-A and H3.1 are compared at single amino acid resolution. Dashes have been added at the relative position for H3.1. Known posttranslational modifications are mapped onto the sequence of both histones. Amino acid numbering begins with the N-terminal methionine as amino acid number 1 , which is usually cleaved from the mature CENP-A protein. Binding sites of HJURP, CENP-N and CENP-C are highlighted on the CENP-A sequence, as well as the CENP-A-CENP-A dimerization domain (labeled nucleosome self-association). (B) Space-filling models of the CENP-A nucleosome as well as a CENP-A-CENP-A dimer from different perspectives. Highlighted amino acids are shaded to match the colors of the binding sites mapped in A. Two additional, non-conserved residues (R80, G81) in CENP-A constitute a bulge relative to $\mathrm{H} 3$. 
A
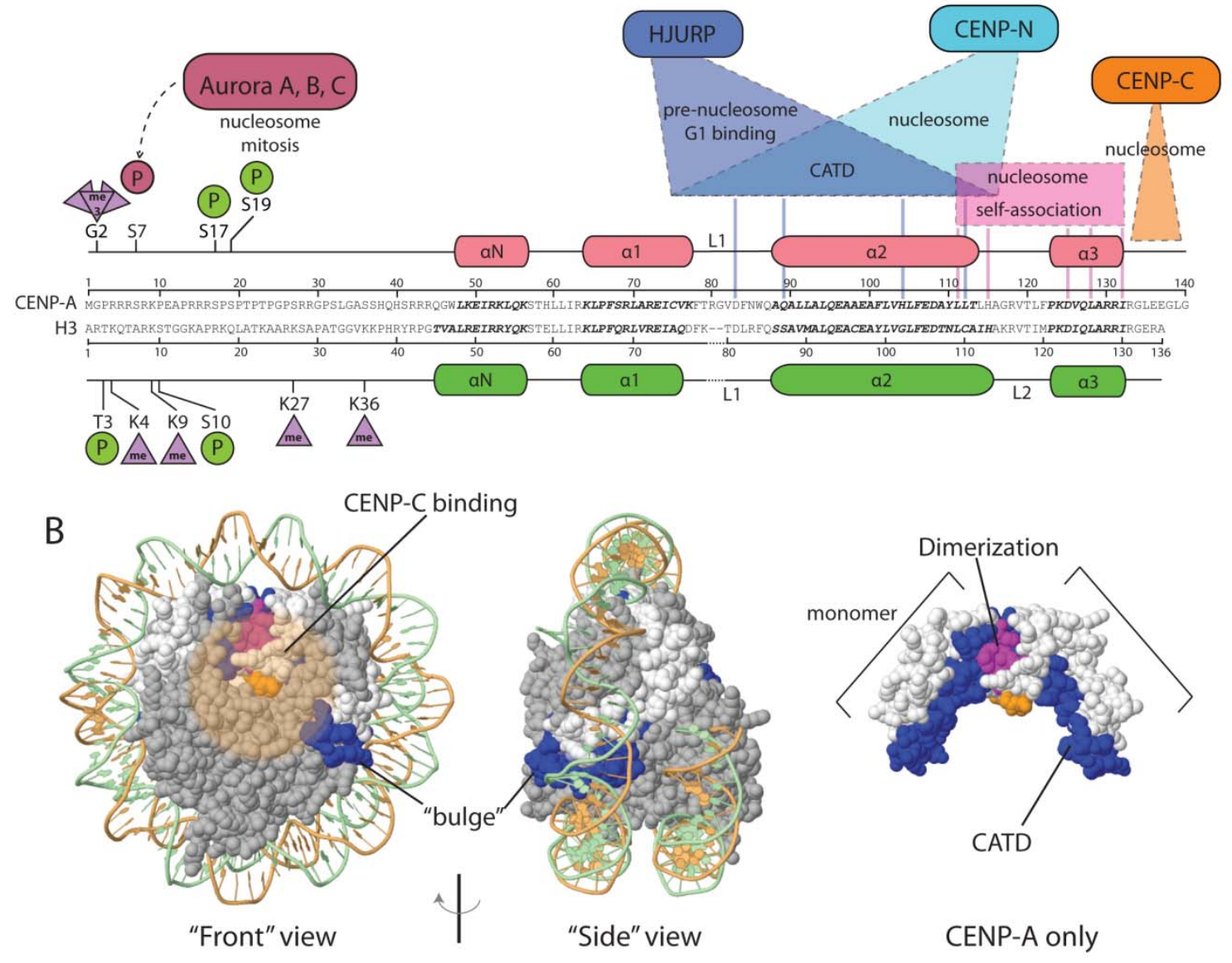

Figure 1-1

Figure designed by A.O. Bailey 


\section{Centro-chromatin: Epigenetic context of CENP-A}

A consensus of immunofluorescence microscopy data on stretched interphase chromatin shows that centromeres contain alternating stretches of $\mathrm{H} 3$ and CENP-Acontaining nucleosomes, which is a quality conserved from flies to humans [41-43]. The amino-terminal tails of the interspersed histone H3 stretches are enriched for dimethylation on Lys4, Lys9 and Lys36 (H3K4me2, H3K9me2 and H3K36me2) (Figure $1-2)[43,44]$. The pattern of centromeric histone post-translational modifications is different from that of general chromatin as well as that of pericentric heterochromatin and does not adhere to the characteristic "activating" or "silencing" patterns. The perturbation of histone marks within the centromeres of human artificial chromosomes (HAC) results in a loss of HAC stability, loss of centromere-specific proteins and an inhibition of the CENP-A deposition pathway [44-47]. This suggests that the unique combination of histone modifications present in centromeric chromatin may be important for centromere function and propagation.

Another centromeric, chromatin-associated complex has also been proposed that includes members of the CCAN, the CENP-T/W/S and X complex [48]. Each of these proteins contains a histone fold domain. Histone folds are not only found in histones, but are also found in several transcription factor complexes [49]. The members of the CENP$\mathrm{T} / \mathrm{W} / \mathrm{S} / \mathrm{X}$ complex use their histone folds to form a heterotetramer, which has structural similarities to transcription factor complexes as well as the histone $\mathrm{H} 3-\mathrm{H} 4$ heterotetramer. Mutations in any of the tetramerization domains in this complex results in failed mitoses in vivo, suggesting that this complex is absolutely required for kinetochore formation in 
chickens and humans [48]. The CENP-T/W/S/X complex binds and protects 100 base pairs of DNA from nuclease digestion in vitro. While these data suggest that CENP$\mathrm{T} / \mathrm{W} / \mathrm{S} / \mathrm{X}$ may form a nucleosome-like structure at centromeres; CENP-T/W/S/X may simply bind to centromeric DNA, albeit in a discrete complex.

The stability of the CENP-T/W/S/X complex is very different from that of CENPA, which is stable throughout the cell cycle and is completely retained through S-phase. Recruitment of CENP-T and -W occurs during late S-phase or G2 [50, 51]. The CENP$\mathrm{T} / \mathrm{W}$ dimer does not remain stably-bound to centromeres, but is instead completely replenished upon each new cell cycle [51]. The CENP-T/W/S/X complex appears to be interspersed between CENP-A domains in stretched chromatin fibers, and immunoprecipitations of the complex from MNase treated extracts pull down histone $\mathrm{H} 3$ $[50,52]$. This suggests that the CENP-T/W/S/X complex may couple the kinetochore to the $\mathrm{H} 3$ containing domains of centromeric chromatin $[50,52]$.

During mitosis, the higher-order organization of the centromere is speculated to resemble a cylinder or a multi-layered boustrophedon $[41,52]$. CENP-A occupies $10 \%$ of the DNA at the primary constriction, in a condensed space at the distal, kinetochorefacing aspect of centromeres [53]. A self-organization model has been proposed to generate this three-dimensional centromeric chromatin structure. In such a model, centromeric chromatin folds into a specific three-dimensional structure that facilitates kinetochore formation above the external CENP-A clusters while the interspersed H3 regions are excluded as a looped or coiled structure to form the inner centromere below. 
Consistent with this idea, the N-terminal tails of $\mathrm{H} 3$ nucleosomes have been shown to dictate the three dimensional folding of polynucleosome arrays [54]. CENP-A and $\mathrm{H} 3 \mathrm{~N}$-terminal tails are vastly divergent. In vitro folding experiments show that arrays of CENP-A nucleosomes were found to fold into more condensed, higher-ordered structures than $\mathrm{H} 3$ nucleosome arrays [31]. The differences in histone posttranslational modifications found in the inner and outer centromere and pericentric regions may influence this property. Therefore, histone variant specific protein-protein interactions may confer a way for three-dimensional folding instructions to be laid out in the two dimensional organization of the centromere. 
Figure 1-2. The 3D organization of the human centromere.

Centromere and pericentromere chromatin organization depicted in interphase (1D model - left) and in mitosis (2D model - right). Histone H3 and CENP-A post-translational modifications are notated per cell cycle position, black for constitutive modifications and red for mitosis specific posttranslational modifications. CENP-A modifications are numbered including the initiating methionine. In the 2D model, background shading distinguishes the organization of the centromere: pericentromere (dark grey), inner centromere (light grey), and outer centromere (pink, green/red). 
Interphase

Mitosis

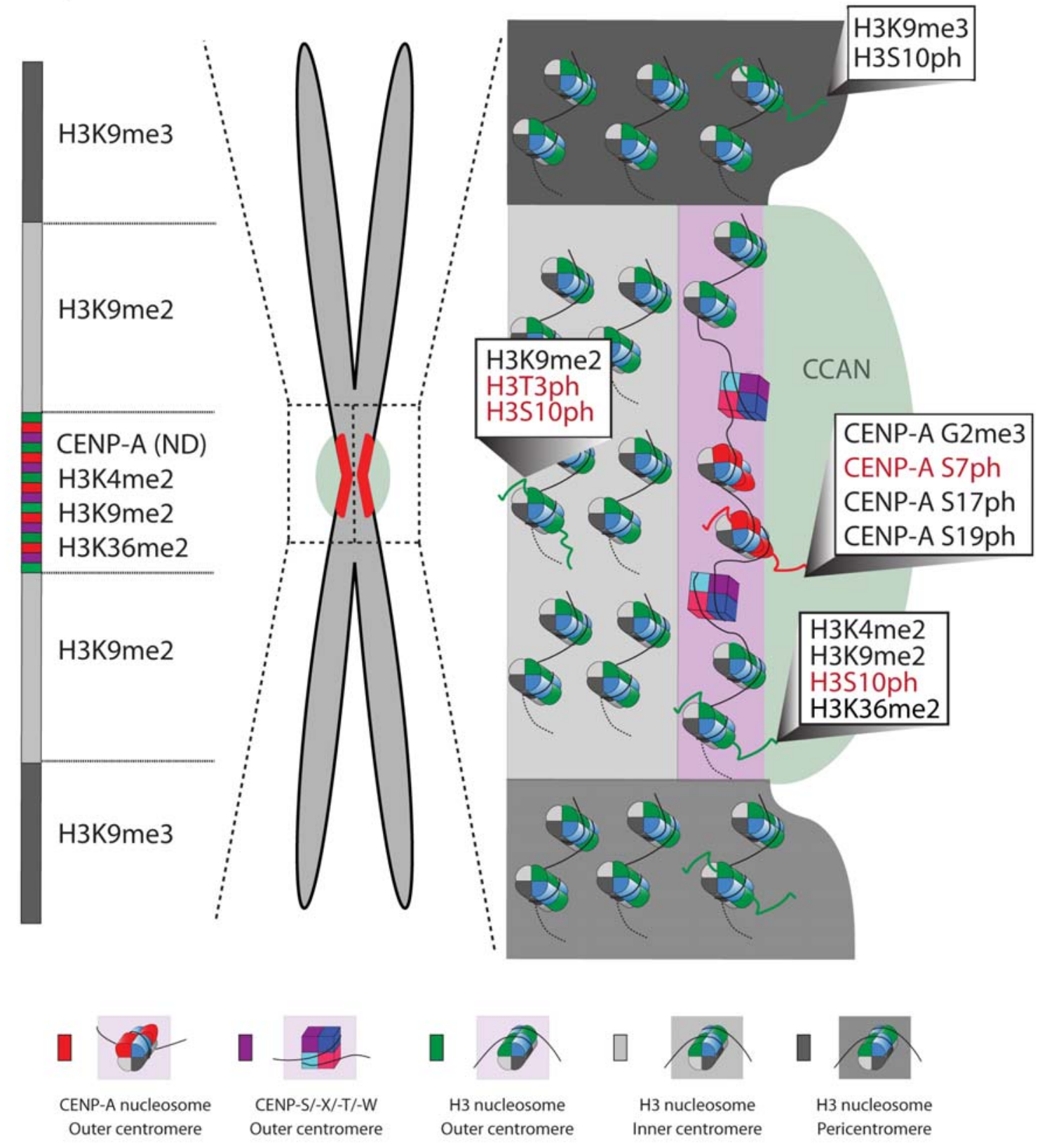

Figure 1-2

Figure designed by A.O. Bailey 


\section{Influences of heterochromatin in centromere specification}

Regional centromeres are consistently organized such that a central CENP-Acontaining region is flanked by pericentric heterochromatin (Figure 1-2). In humans, pericentric heterochromatin contains nucleosomes that are trimethylated on lysine 9 of histone $\mathrm{H} 3$ (H3K9me3), while H3K9me2 chromatin is interspersed with CENP-A inside the centromere region [43]. Pericentric heterochromatin is a repressive chromatin structure, where the $\mathrm{H} 3 \mathrm{~K} 9$ trimethylation acts as a signal for the recruitment of the chromodomain protein, HP1 [55, 56].

Heterochromatin formation in S. pombe is required for de novo centromere formation [57]. Pericentromeric regions are established through the RNAi pathway. H3K9me3 and Chp1 mediate binding of the RITS complex, which in turn recruits RNAdependent polymerase complex (RDRC) mediated transcription of double stranded RNA. Dicer then processes the double stranded RNA to generate siRNAs that help facilitate centromere silencing. In a positive-feedback loop, the methyltransferase Clr4 is locally recruited by the RNAi pathway and reinforces the trimethylation mark on H3K9 [58]. In plasmid based de novo centromere formation assays, when heterochromatin formation is inhibited on flanking regions, de novo CENP-A nucleosome deposition cannot occur. However, when the siRNA requirement is bypassed by direct targeting of the Clr4 methyltransferase, CENP-A nucleosomes are deposited demonstrating that the key requirement for de novo CENP-A deposition is the activity of Clr4 [59].

In addition, neocentromere formation in other experimental systems appears to prefer sequences that are in close proximity to heterochromatin, perhaps reflecting the 
need for pericentric heterochromatin in de novo centromere formation. When neocentromeres are induced by the removal of endogenous centromere sequences on chromosome 1 in $S$. pombe, neocentromeres most often arise near telomeric regions, where H3K9 methylation is present [16]. Likewise, in D. melanogaster, overexpressed CID/CenH3 forms islands throughout the length of the chromosomes, but it appears that ectopic centromere formation is biased to regions where heterochromatin and euchromatin are in close apposition [60]. It seems that this boundary element may make a more permissive structure for de novo CID/CenH3 deposition. It is not clear if these same modifications influence neocentromere formation in humans, as the consistent localization of $\mathrm{H} 3 \mathrm{~K} 9 \mathrm{me} 3$ regions nearby sites of neocentromere formation has so far not been observed [61]. 


\section{The CCAN: Coupling Chromosomes to the Kinetochore}

The CCAN forms a bridge between centromeric chromatin and the mitotic kinetochore. Two distinct components of the CCAN have been shown to "read" the unique structure of the CENP-A nucleosome. CENP-C and CENP-N both interact with the CENP-A nucleosome, but in different ways. CENP-C is recruited to centromeres via an interaction with the extreme carboxyl terminus of CENP-A (Figure 1-1) [62, 63]. Replacement of the extreme C-terminus of histone $\mathrm{H} 3$ with the last six amino acids of CENP-A is sufficient to recruit CENP-C in vitro and in Xenopus extracts [62, 63]. However, the primary sequence of the CENP-A carboxyl terminus is not conserved between yeast, flies, zebra fish and humans. In addition, a chimeric histone H3 containing

the CATD (H3 ${ }^{\text {CATD }}$ ), which lacks the C-terminus of CENP-A, was still sufficient to recruit CENP-C to centromeres at endogenous levels in human cells [64]. Therefore, it remains to be demonstrated whether recruitment of CENP-C through the C-terminus of CENP-A is a conserved method of CENP-A recognition, or whether multiple mechanisms link CENP-A and CENP-C.

The interaction of CENP-N with CENP-A is through the CATD of CENP-A [65]. CENP-N selectively interacts with CENP-A in its nucleosomal form, suggesting that it recognizes a structural aspect of CENP-A that is only found in the intact nucleosome [62, 63, 65]. Both CENP-C and CENP-N appear to prefer nucleosomal CENP-A in vivo as well, as neither of these two proteins is found in prenucleosomal CENP-A fractions [21, $66]$. 
CENP-C and -T also interact with proteins of the kinetochore. A major microtubule-binding complex of the kinetochore is the KMN network which consists of KNL-1, the Mis12 complex, and the Ndc80 complex, which is recruited by the Nterminus of CENP-C [67-69]. The amino terminus of CENP-T extends far beyond its Cterminal histone fold domain's incorporation within centromeric chromatin, and its Nterminus interacts with the Ndc80 complex in the outer kinetochore [70-72]. A third CCAN/KMN network interaction point involves a complex of CENP-H, -I, and -K which appears to be recruited distally to CENP-C and $-\mathrm{N}$ and participates in kinetochore formation through direct binding to KNL-1 [73]. When CENP-T and CENP-C are targeted to a non-centromeric locus using the LacO/LacI system, they are sufficient to assemble a functional kinetochore, including mitotic checkpoint signaling, which does not require CENP-A at this artificial locus [71].

Although the KMN network can directly bind microtubules through KNL-1 and through the Ndc80 complex, the CCAN may also play a direct and dynamic role in microtubule binding. During typical kinetochore oscillations, CENP-H/I show dynamic enrichment at kinetochores coupled to growing versus shrinking microtubules [74]. In addition, a complex involving CENP-O (Mcm21R), -P, -Q, and -U (CENP-50) appears to play a role in regulating the quality of microtubule attachments to kinetochores and is essential for recovery from experimentally induced spindle damage in vivo [75]. Human CENP-Q can directly bind microtubules in vitro, so it is speculated to serve as the microtubule-binding component in the CENP-O/P/Q/U sub-complex [74]. Phosphorylation of CENP-U by Aurora B is required for spindle damage recovery [75]. 
Because CENP-U is regulated by Aurora $\mathrm{B}$, the role of the CENP-O/P/Q/U sub-complex, and thereby the entire CCAN, is not simply binding kinetochore components, but rather playing an active role in generating correctly formed microtubule attachments. 


\section{Maintaining Centromere Identity through DNA Replication}

Replication of the genome necessitates that nucleosomes be distributed to each newly synthesized sister chromatid and requires the stable propagation of CENP-A and many other histone modifications. Replication of general chromatin includes the incorporation of new histone H3.1 containing nucleosomes as well as the re-incorporation of histones from pre-existing nucleosomes $[76,77]$. As the replication fork passes through chromatin, pre-existing nucleosomes are distributed among the daughter strands. Then, newly synthesized histones are deposited into both strands to make up for the dilution of histones that occurs during the replication process.

At the centromere, previously incorporated CENP-A nucleosomes are stably retained through S-phase [78]. Each CENP-A domain parses its CENP-A nucleosomes between the two daughter strands. Immunofluorescence studies of replicated chromatin fibers show that daughter strands have the same number of CENP-A blocks, but the blocks are one half of the intensity of pre-S-phase centromeres [79]. How CENP-A nucleosomes are stably transited across the replication fork and whether this mechanism is similar to that used by $\mathrm{H} 3$-containing nucleosomes remains unknown.

However, newly synthesized CENP-A is not deposited during S phase, and minimal availability of the CENP-A protein may help to preclude CENP-A deposition during replication. Overexpression of CENP-A can lead to the misincorporation of CENP-A into non-centromeric chromatin, suggesting that high levels of CENP-A can overtax the mechanisms that restrict CENP-A deposition to centromeres [80-82]. While canonical histone levels increase by early $\mathrm{S}$ phase to allow for the massive deposition of 
new nucleosomes, CENP-A mRNA levels do not rise until mid S-phase leading to the accumulation of newly synthesized CENP-A protein in G2, after replication has finished [83-85]. As such, high levels of CENP-A are not available for deposition during replication, and G2 centromeres contain only half of the amount of CENP-A molecules that were present in the parental centromere prior to replication.

Without placing new CENP-A nucleosomes during replication, cells may leave gaps in centromeric loci resulting in only partial nucleosome protection. Alternatively, H3.1/3.3-containing nucleosomes (or an alternative complex) may occupy these CENP-A deficient regions. Recent data suggests that histone H3.3 nucleosomes may serve to fill in the gaps left by CENP-A distribution at the centromere during DNA replication, and these H3.3 nucleosomes are then exchanged in G1 when newly CENP-A nucleosomes are deposited [79]. It is unclear whether the deposition of $\mathrm{H} 3.3$ at the centromere is through the replication machinery or dependent on centromeric transcription. Regardless, it suggests that active displacement of a "placeholder" through a chromatin-remodeling event may be necessary in order to make room for newly synthesized CENP-A nucleosomes in G1.

An alternative model is that old CENP-A nucleosomes are split between the two daughter chromosomes generating either a "hemisome" consisting of only one copy of each histone or a heterotypic nucleosome containing one copy of CENP-A and one copy of H3.1/3.3. There is some evidence to support the existence of a hemisome-like particle via crosslinking studies as well as atomic force microscopy, in which the height of the CENP-A nucleosome particles are one half the size of the canonical H3 nucleosomes [24, 
25]. In addition, fluorescent molecule FRET experiments in budding yeast and humans indicate that CENP-A nucleosomes may switch from hemisomes to octamers at specific times in the cell cycle $[86,87]$. In humans, the data suggests that the nucleosomes become octameric at the G1/S boundary [87]. This would provide a model for propagation of CENP-A particles during S phase without deposition of newly synthesized CENP-A. However, many other studies, including crystal structure analysis, indicate a definite presence and structural role for the traditional octamer.

Another level of complexity lies in the distribution of various CCAN components during S phase. The CCAN is a large complex of centromere proteins (CENPs) that are constitutively localized at the centromere throughout the cell cycle [21]. While these proteins are continually present at centromeres, their localization is dynamic, with varied exchange profiles $[88,89]$. CENP-N has a rapid exchange rate throughout the cell cycle, allowing it to dissociate as the replication fork passes (Figure 1-3). However, during late S phase, centromeric levels of CENP-N increase as the exchange rate drops [89]. This suggests that CENP-N is free to diffuse before replication, but afterwards loads onto centromeric chromatin in a more stable manner. This may enable CENP-N to mark centromeres that have completed replication.

The CENP-T/W/S/X complex remains stably associated with centromeric DNA throughout most of the cell cycle, but becomes dynamic in late $\mathrm{S}$ phase and completely turns over, suggesting that the CENP-T/W/S/X complex is disrupted by the replication fork and reassembles after it has passed [51]. In contrast, CENP-C is highly dynamic throughout the cell cycle but becomes stably associated with centromeres during $\mathrm{S}$ phase 
and mitosis [88]. CENP-C interacts directly with CENP-A nucleosomes, meaning that CENP-C may pass the replication fork in a similar manner. How CENP-A nucleosomes transit the replication fork during S-phase is an extremely interesting yet poorly understood aspect of centromere inheritance, and it is fascinating to consider the possible roles of the various CCAN proteins in this process. 
Figure 1-3. Replication of centromeric chromatin and the $S$ phase dynamics of the CCAN.

Centromeric chromatin is replicated in $\mathrm{S}$ phase concurrently with general chromatin. $\mathrm{S}$ phase dynamics of the most CENP-A-chromatin proximal CCAN proteins: CENPT/W/S/X complex, CENP-C and CENP-N are also shown. Red arrows symbolize dissociation from the centromere. The green arrow symbolizes possible replication fork passage. Black arrows symbolize loading of new CENPs. (A) Existing CENP-A nucleosomes are allotted to each daughter strand, but no new CENP-A nucleosomes are added during S phase. New H3.1/H3.3 nucleosomes may serve as placeholders during replication-coupled dilution of existing CENP-A nucleosomes at the centromere (yellow nucleosomes). CENP-C is stably associated with centromeres in S phase and may track with CENP-A nucleosomes across the replication fork. (B) As the replication fork passes, $\mathrm{CENP}-\mathrm{T} / \mathrm{W} / \mathrm{S} / \mathrm{X}$ complexes are turned over every cell cycle and load during late $\mathrm{S}$ phase. CENP-N localization is dynamic throughout the cell cycle, but loads to maximal levels during $\mathrm{S}$ phase. 


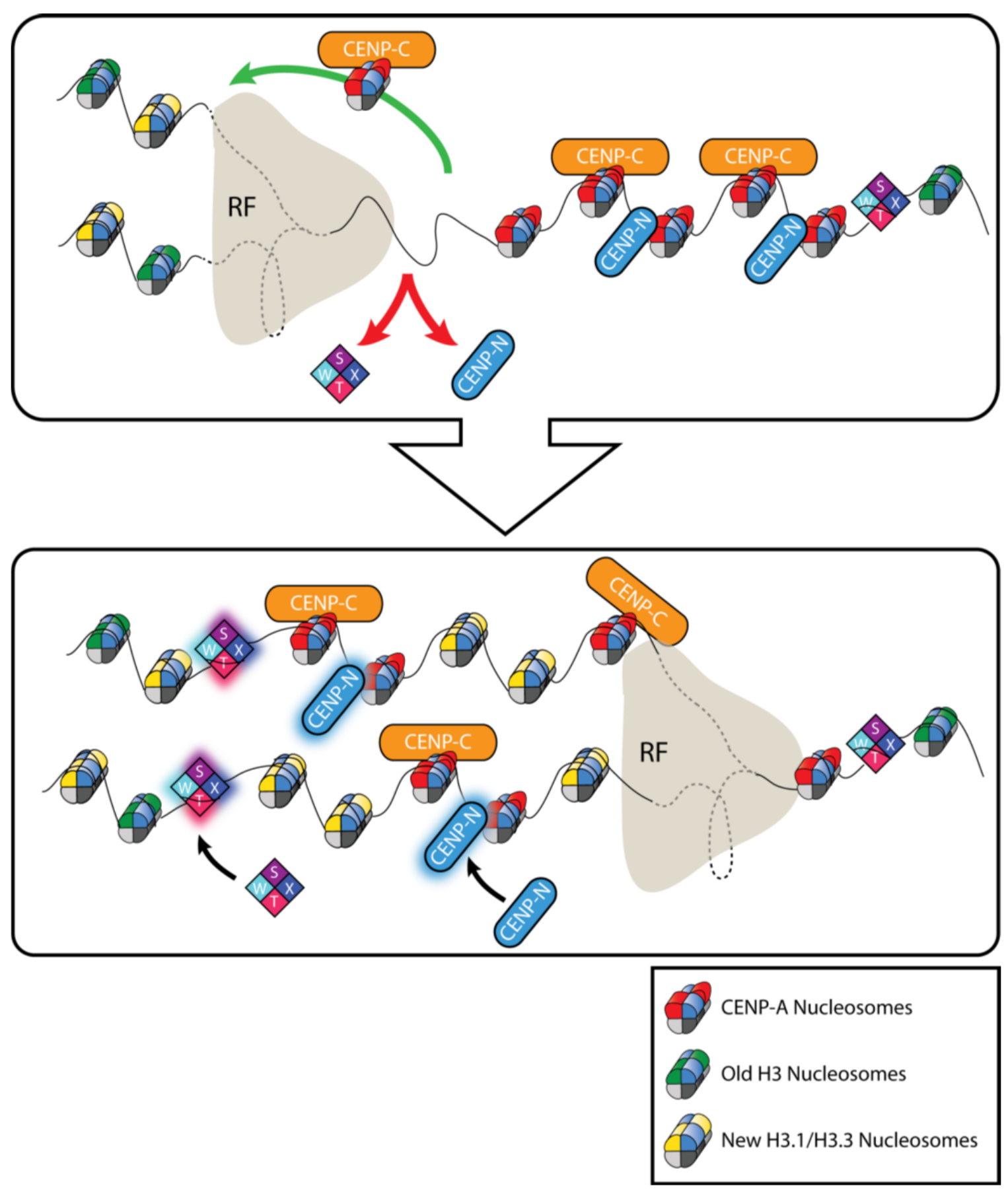

Figure 1-3 


\section{The CENP-A Deposition Pathway}

In order for centromeres to be stably inherited across many generations, new CENP-A nucleosomes must be assembled specifically at the site of the pre-existing centromere after each round of DNA replication. For human CENP-A containing nucleosomes, this process occurs during G1, after mitotic exit. The CENP-A deposition pathway can be broken down into three basic stages that involve distinct protein complexes: initiation, deposition, and maintenance. At the correct moment in the cell cycle, the location of the centromere must be sensed and the underlying chromatin modified to or maintained in a permissive state for CENP-A deposition. Once this occurs, CENP-A-specific assembly factors associate with the centromere and allow for CENP-A deposition. Finally, through chromatin remodeling and maturation processes, centromeric chromatin is fully stabilized.

\section{Initiation of CENP-A deposition: Key players}

In human cells, the deposition of CENP-A occurs during G1, after cells exit mitosis [78]. Therefore, initiation must occur prior to CENP-A loading. The earliest recognized step in the recruitment of new CENP-A nucleosomes is the association of the Mis 18 complex, which localizes to centromeres in late anaphase, directly after mitotic exit [90]. Mis 18 was first discovered in fission yeast by a temperature sensitive screen for mutants that missegregated their chromosomes [91]. Along with Mis 16 mutants (homolog of the human proteins RbAp48 and RbAp46), the Mis 18 temperature sensitive mutants resulted in the missegregation of chromosomes during mitosis due to a reduction 
of centromeric Cnp1, the CENP-A homolog in S. pombe [91]. This indicated that Mis16 and Mis18 were both required for the deposition of Cnp1-containing nucleosomes at the fission yeast centromere.

Through sequence homology searches, two human homologs of the Mis 18 protein were discovered: Mis18 $\alpha$ and Mis18 [90]. Pull downs of both Mis18 $\alpha$ and Mis18 $\beta$ from chromatin fractions confirmed a physical interaction between the two Mis18 proteins as well as with the human homologs of Mis16, RbAp48 and RbAp46 [90]. In addition, an uncharacterized protein, termed Mis18 Binding Protein 1 (Mis18BP1), was also found to interact with chromatin-associated Mis18 $\alpha$ and Mis18 $\beta$ [90]. Concurrently, an RNAi screen in C. elegans discovered a homolog of Mis18BP1 (named KNL-2) that was also required for CENP-A centromeric localization in nematodes; thus providing further evidence of the conserved nature of these proteins [92].

RNAi depletion experiments in human cells showed that Mis18 $\alpha$, Mis18 $\beta$, and Mis18BP1 were dependent upon each other for localizing to the centromere [90]. In addition, depleting all three proteins as well as RbAp48/46 confirmed that the entire complex was required for the deposition of newly synthesized CENP-A at the human centromere $[90,91]$. In summary, the human Mis 18 complex was found to consist of Mis18 $\alpha$, Mis18ß, Mis18BP1, RbAp48 and RbAp46, which were all required to deposit new CENP-A at centromeres. Although, little was initially known regarding the mechanisms by which this complex influenced downstream CENP-A deposition, several aspects will be addressed in the following chapters. 


\section{Initiation of CENP-A deposition: Temporal regulation}

In many organisms, it has been shown that the deposition of newly synthesized CENP-A nucleosomes can occur via a replication-independent process unlike the assembly of canonical H3.1 nucleosomes, which is concurrent with DNA synthesis [84]. As described above, human CENP-A is not available for deposition during DNA replication. CENP-A mRNA and protein levels are not maximal until the end of S phase, after the majority of centromeric DNA has already completed replication [84, 85]. Instead, human cells and D. melanogaster embryos load new CENP-A nucleosomes in G1 only after mitotic exit $[78,93]$. In D. melanogaster S2 cells, deposition occurs slightly earlier during mitosis [94]. In an independent genome-wide RNAi screen in $D$. melanogaster, depletion of cyclin A and Rca1, an inhibitor of the Chd1-APC complex, caused a direct loss of CID at centromeres $[95,96]$. Therefore, although the timing between systems differs slightly, data greatly suggest that CENP-A deposition is regulated by cell cycle progression.

Since human CENP-A deposition only occurs in G1, it is hypothesized that the proteins involved in CENP-A deposition must be regulated by the cell cycle. Progression through the cell cycle is orchestrated by the cyclical accumulation and destruction of the cyclin proteins and their interactions with the various cyclin dependent kinases (Cdks) $[97,98]$. Throughout G1 and S phase, cyclin A and cyclin B1 accumulate, which results in increased Cdk1 and Cdk2 activity. By the G2/M transition point, Cdk activity levels are maximal, ensuring that the vast majority of Cdk substrates are maintained in a phosphorylated state. Cdk activity levels remain elevated by the spindle assembly 
checkpoint until all sister chromatids are bioriented at the metaphase plate. After the SAC is satisfied, the cyclins are rapidly degraded by the anaphase-promoting complex and the cell enters G1, with minimal Cdk activity.

This dearth of Cdk activity at anaphase onset coincides with the deposition of newly synthesized CENP-A (Figure 1-4) [78, 93]. Many of the proteins required for the deposition of CENP-A at centromeres share the localization pattern of associating with centromeres after the exit from mitosis, when Cdk activity is low $[66,83,90,91]$. This suggests that there may be a Cdk controlled mechanism that directly prevents CENP-A deposition in S phase when Cdk activity is high, but allows for CENP-A assembly into centromeric chromatin after the exit from mitosis, when Cdk activity is low (Figure 1-4). 
Figure 1-4. Overview of the cell cycle control mechanism of CENP-A deposition.

The deposition of CENP-A is tightly coordinated with the cell cycle. Chromosome schematics show the stepwise change in CENP-A protein levels at the centromere and the apparent localization dynamics of CENP-A deposition factors. (A) Starting at the exit from mitosis, each daughter centromere possesses one-half of the full complement of CENP-A nucleosomes (light pink oval). Cyclins are rapidly degraded following mitotic exit, and Cdk activity dramatically drops (dark blue gradient). The Mis18 complex associates with centromeric chromatin directly after mitotic exit. (B) HJURP appears to be recruited soon after and deposits newly synthesized CENP-A nucleosomes (light pink to red gradient oval). (C) In mid to late G1, the RSF complex (Rsf1-Snf2h) and MgcRacGap interact transiently with centromeres to stabilize newly assembly CENP-A nucleosomes and generate mature centromeric chromatin (red oval). By the G1/S phase transition, Cdk activity levels increase again. (D) During S phase, CENP-A nucleosomes are parceled to each daughter centromere (two light pink ovals). 


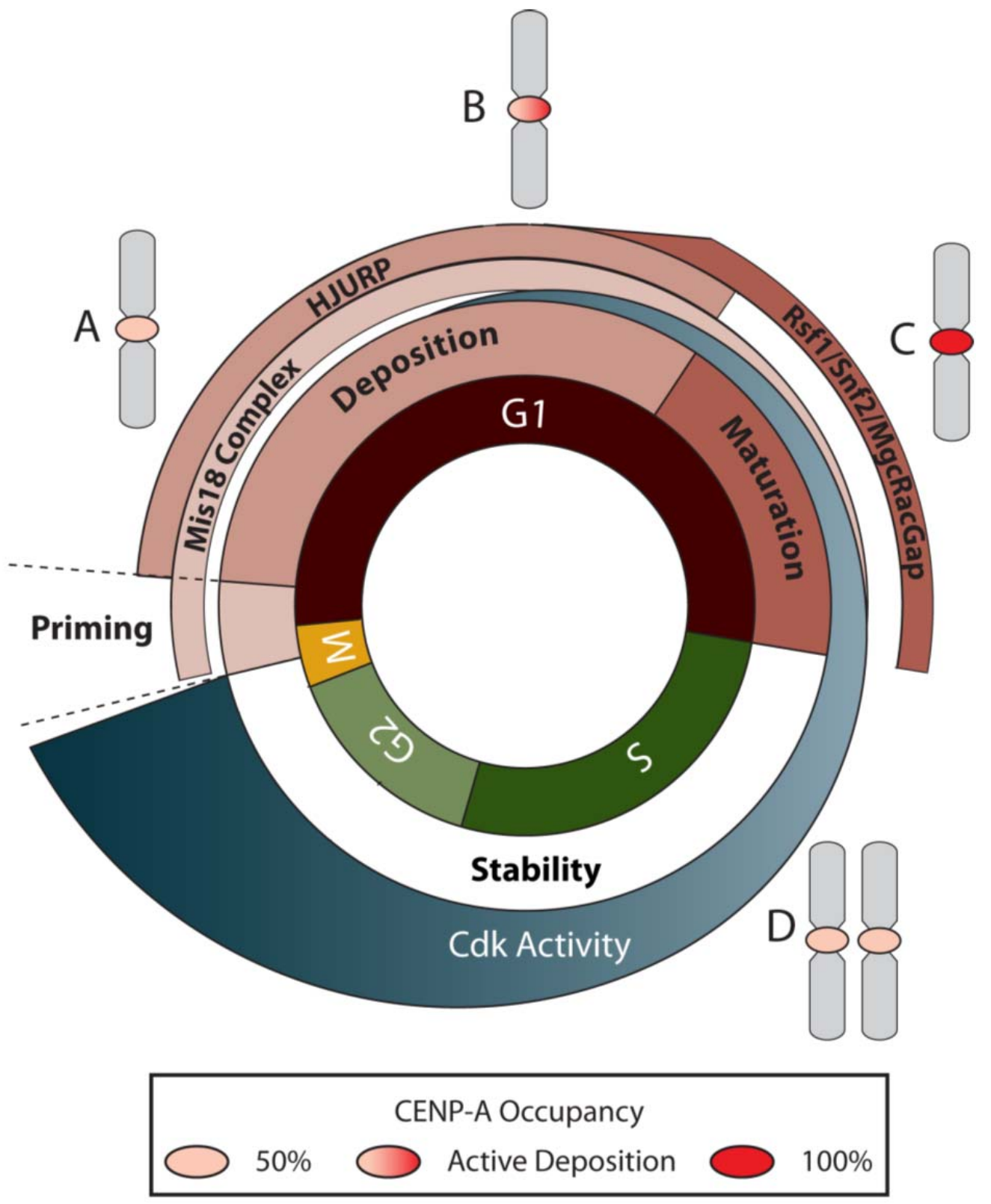

Figure 1-4 


\section{Initiating CENP-A deposition: Spatial regulation}

CENP-A deposition must be spatially regulated to occur only at the designated centromere locus. This may be achieved by coupling the CENP-A deposition machinery to constitutive centromere proteins. Recent experiments have identified a physical interaction between the CCAN protein, CENP-C, and the Mis 18 complex $[99,100]$. While centromeric localization of CENP-C was shown to be dependent upon the presence of CENP-A nucleosomes, several studies in D. melanogaster cells determined that CENP-C is required for efficient deposition of CENP-A [95, 101, 102]. In egg extracts, one isoform of Xenopus Mis18BP1 (M18BP1) was dependent upon CENP-C to localize to metaphase centromeres [100]. Recent studies in mice suggest a similar interaction between Mis18BP1 and CENP-C [99]. Of the various CCAN proteins assayed, only CENP-C co-localized with Mis18BP1, when it was targeted to a chromatin locus outside of the endogenous mouse centromere [99]. As in Xenopus, this colocalization was found to be mediated through a physical interaction with Mis18BP1 and a C-terminal portion of CENP-C $[99,100]$.

This proposes a model of reinforcement whereby new CENP-A deposition is reinforced at an existing centromere by the presence of the CCAN protein, CENP-C, which directs the localization of the CENP-A assembly factors. Several observations support this idea of a feed-forward mechanism to ensure continual enrichment of newly synthesized CENP-A at active centromeres. Overexpression of CENP-A (CID) in flies results in its mislocalization throughout chromatin; however, only a subset of regions containing the mislocalized CENP-A develop into active centromeres that recruit CCAN 
proteins and downstream kinetochore components [81, 82]. It may be that the recruitment of CCAN proteins at non-centromeric loci is minimal, possibly due to limiting protein levels. Therefore, the continual recruitment of new CENP-A to non-endogenous CENP-A foci may be limited and centromeric CENP-A is continually replenished because of the presence of bound CCAN proteins.

Another physical characteristic that may direct CENP-A deposition at centromeric sites is the overall architecture of a post mitotic centromere. Human CENP-A deposition occurs immediately following mitosis, and the hypercondensed state of chromatin in early anaphase may be the most efficient substrate for CENP-A deposition. Human condensin I and condensin II are multiunit complexes that function to condense chromosomes during mitosis [103]. Experiments in several organisms suggest that chromatin condensation may be important for the efficient deposition of CENP-A nucleosomes. At the budding yeast point centromere, condensin depletion results in decreased localization of Cse4 to centromeric chromatin [104]. Experiments using Xenopus egg extracts demonstrated that condensin II is required for efficient CENP-A deposition [105]. SMC2 depletion by siRNA in human cells leads to decreased recruitment of new CENP-A nucleosomes to centromeres [106]. Condensin association with chromosomes peaks in anaphase, placing it directly prior to the association of the Mis 18 complex with centromeres in late anaphase $[90,107]$. While these experiments suggest a connection between CENP-A deposition and condensin, the function of condensin may be simply to maintain a three-dimensional structure that is required to facilitate CENP-A deposition. 


\section{Priming the centromere for CENP-A deposition}

The exact function of the Mis 18 complex at centromeres largely remains a black box in the understanding of the centromere lifecycle. Current research in the field centers on the hypothesis that the Mis 18 complex primes centromeres for CENP-A deposition by recruiting chromatin modifying activity to the centromere in order to generate or maintain a permissive state for CENP-A deposition [90, 91].

In fission yeast, temperature sensitive mutants of Mis 16 and Mis18 show a significant increase in the acetylation of centromeric $\mathrm{H} 4$ at the inner centromere repeats (cnt1 and imr1) [91]. Although, where fission yeast Mis 18 seems to antagonize histone acetylation, the human Mis18 complex appears to promote acetylation. Cells depleted for members of the Mis 18 complex, lose the deposition of newly synthesized CENP-A at centromeres; however, treating those cells concurrently with the HDAC inhibitor, trichostatin A (TSA), rescues CENP-A deposition [90]. Global inhibition of HDAC activity with TSA would increase the acetylation status of the entire genome and theoretically the centromere as well.

Consistent with these observations, targeting the histone acetyltransferase activity of P300 or PCAF to a human artificial chromosome is sufficient to induce CENP-A deposition [47]. In addition, an increase in centromeric $\mathrm{H} 3$ acetylation can be seen in early G1, which correlates with the localization of the Mis 18 complex at centromeres. However the identity of the endogenous histone acetyltransferase responsible for this activity is not known. Since an artificial increase in centromere acetylation seems to 
bypass the requirement for the Mis18 complex in CENP-A deposition, this argues that the human Mis18 complex may function by affecting the centromeric histone acetylation.

The Mis 18 complex may also affect CENP-A deposition by altering epigenetic modifications of DNA. The DNA methyltransferases, DNMT3A and DNMT3B, interact with centromeric chromatin through interactions with Mis18 $\alpha$ and CENP-C $[108,109]$. Conditional knockout studies in mouse embryonic fibroblasts found that Mis $18 \alpha$ loss resulted in a reduction of centromeric DNA methylation [109]. Disrupting DNMT3A/B decreased the methylation of centromeric DNA and lead to a significant decrease the level of CENP-A at the centromere. However, mouse ES cells in which DNMT1, DNMT3A and DNMT3B were disrupted by gene targeting, replicate and divide normally without chromosomal instability or gross segregation errors [110]. It is not clear whether DNA methylation is actually part of the specific CENP-A recruitment pathway or whether disruption of DNA methylation alters the state of centromeric chromatin and leads to downstream problems in CENP-A deposition.

Further evidence to support the role of the Mis18 complex in affecting the histone modification state of centromeric chromatin is the fact that the proteins of the Mis18 complex have several ties to chromatin remodeling and modifying complexes. Mis18BP1 contains a SANT (Swi3-Ada2-NCoR-TFIIIB) domain as well as a SANT-Associated (SANTA) domain $[90,111]$. The SANTA domain was found in silico as a domain that characteristically is present in proteins which also contain a SANT domain [111]. In the human proteome, the SANTA domain has been identified exclusively in Mis18BP1. The function of the SANTA domain is currently unknown, although, the conserved 
hydrophobic residues are proposed to be involved in protein-protein interactions, possibly mediating contacts with its various binding partners at the centromere [111].

As its full name implies, the Swi3-Ada2-NCoR-TFIIIB (SANT) domain is found in a variety of chromatin remodeling and modifying complexes including the remodeler, SWI/SNF, and the SAGA histone acetyltransferase (HAT) complex [112]. SANT domains are made up of roughly 50 amino acids that form three alpha helices in a helixturn-helix motif, similar to the DNA binding domain in c-Myb [113]. The Myb domain in the proto-oncogene, c-Myb, has been shown to bind DNA in a sequence specific manner [114]. However, analysis of the crystal structure of the SANT domain in Xenopus ISWI shows that the amino acid residues responsible for the sequence specific DNA interactions in c-Myb are not conserved in all SANT domains [113].

Other SANT domains mediate protein-protein interactions to recruit and activate additional binding partners in order to generate a fully functional chromatin-modifying complex. Examples include HDAC3 in the SMRT and N-CoR co-repressor complexes as well as the HAT activity of the SAGA complex [115-117]. In addition, RbAp48 or RbAp46 are common to several known histone modifying and remodeling complexes $[118,119]$. The presence of RbAp48 and RbAp46 as well as the domain architecture of Mis18BP1 give credence to the hypothesis that the Mis18 complex is capable of recruiting chromatin-modifying activity to the centromere. 


\section{Deposition of CENP-A}

The ultimate goal of the centromere specification pathway is the deposition of new CENP-A nucleosomes. Nucleosome assembly is facilitated by the activity of histone chaperone proteins [76, 77]. Other histone $\mathrm{H} 3$ variants, such as $\mathrm{H} 3.1$ and $\mathrm{H} 3.3$, utilize unique chaperone proteins in order to facilitate distinct timing and location of deposition $[76,77]$. Despite vast differences in centromere organization between budding yeast and humans, these organisms all employ a related chaperone, known as HJURP (Holliday junction recognition protein) in humans and $\mathrm{Scm} 3$ in yeast, in order to achieve deposition of newly synthesized CENP-A nucleosomes.

CENP-A/H4 and HJURP form a prenucleosomal complex that localizes to centromeres in G1 during new CENP-A deposition $[66,83,105,120]$. This complex is required for new CENP-A deposition and is sufficient to determine the site of centromere formation [18]. Deletion of Scm3 in budding or fission yeast leads to chromosome loss or missegregation events due to defects in Cse4/Cnp1 recruitment [23, 121, 122]. Although Scm3 and HJURP serve similar functions the entirety of their similarity is located within a small, 50 amino acid, region of homology within their N-termini [9]. HJURP is a much larger, $83 \mathrm{KD}$ protein, in humans compared to the $26 \mathrm{KD} \mathrm{Scm} 3$ protein of S. cerevisiae. HJURP was originally identified as a protein that recognizes synthetic Holliday junctions (thus termed Holliday junction recognition protein) [123]. A requirement for complex DNA structures has not been identified in CENP-A deposition. The differences between HJURP and Scm3 may reflect differing mechanisms by which they are recruited to the centromere. Until recently, a direct fly homolog for HJURP had not been identified; 
however, the localization of the CALI protein and its requirement in CenH3/CID deposition has recently determined CAL1 to be the functional homolog of HJURP/Scm3 $[94,124]$.

The classical role of histone chaperones is to facilitate the deposition of histones into nucleosomes. The deposition of CENP-A nucleosomes is a conserved function of every HJURP/Scm 3 homolog protein. In vitro chromatin assembly assays, using recombinant proteins, show that human HJURP and budding yeast $\mathrm{Scm} 3$ are both sufficient to assemble CENP-A into nucleosomes [18, 125-128]. In each of these cases, HJURP/Scm3 assembles an octameric nucleosome that wraps DNA in a left-handed manner, similar to canonical H3 nucleosomes. In cells, the targeting of HJURP to noncentromeric loci is sufficient to drive the incorporation of CENP-A into chromatin [18]. CENP-A point mutants that affect the CENP-A dimerization interface are able to bind HJURP but cannot be stably assembled into chromatin by HJURP [28]. Heterotypic nucleosomes that contain one copy of CENP-A and histone H3 have been observed as a small fraction of human CENP-A nucleosomes [21]. Since histone H3 uses a similar dimerization interface, mutations in this region would also be expected to eliminate the formation of heterotypic octameric nucleosomes. However, to date no function has been assigned to heterotypic CENP-A-H3 nucleosomes. These data suggest Scm3/HJURP proteins assemble octameric CENP-A nucleosomes at centromeres. Nonetheless, data exists that depicts other sub-octameric forms of centromeric nucleosomes. It is possible that the structure of the CENP-A nucleosome is dynamic throughout the cell cycle, and perhaps may change through downstream remodeling events. 
Recent crystal structures provide interesting insights into the interaction between HJURP/Scm3 and a CENP-A/histone H4 heterodimer in budding yeasts and humans. The CENP-A binding domain (CBD) of HJURP includes the Scm3 homolog domain and forms a long alpha helix followed by a short beta sheet. In all structures, the long alpha helix, within the Scm3 homology domain of HJURP/Scm3, interacts with the CENP-A CATD [129-131]. The CBD of HJURP extends into the region of CENP-A selfassociation and precludes CENP-A/H4 heterotetramer formation; therefore, the HJURP/CENP-A/H4 complex forms a heterotrimer which contains a single copy of each protein $[28,129]$. The formation of the HJURP/CENP-A/H4 prenucleosomal complex excludes tetramer formation and DNA interaction suggesting a step-wise conformational change is required for incorporating CENP-A into centromeres.

Residues outside of the CATD domain of CENP-A also interact with the previously identified TLTY box recognition domain of HJURP/Scm3, although it is unlikely that these resides contribute to specificity $[28,120,129]$. These structures show that although the centromeres of yeast and man are very divergent, the specific interaction between HJURP/Scm3 and a CENP-A/H4 heterodimer is conserved.

\section{Centromeric chromatin maturation}

A growing amount of evidence supports the idea that CENP-A nucleosomes are not fully stable after their initial deposition in early G1, but require additional changes through remodeling complexes and GTP cycling to become fully mature, stable centromeric nucleosomes $[132,133]$. This maturation process occurs after the deposition 
of newly synthesized CENP-A by HJURP and does not affect CENP-A nucleosomes already present at the centromere (Figure 1-4). The RSF complex (Rsf1 and SNF2h) associates with the centromere in mid G1 and confers stability to newly deposited CENPA nucleosomes [132]. In addition, MgcRacGap and Ect2 GTP cycling activity is recruited to centromeric chromatin in late G1 and is also required to stabilize new CENPA nucleosomes [133]. These events occur asynchronously and transiently at only a subset of centromeres during late G1. While the function of this maturation process is not completely understood at this time, RSF and MgcRacGap seem to help generate centromeric chromatin that is sufficiently stable to support its roles throughout the cell cycle, such as serving as the kinetochore platform during mitosis.

\section{Non-coding RNAs, transcription and the centromere}

Mounting evidence suggests that RNA polymerase II (RNAPII) mediated transcription through CENP-A containing chromatin is a conserved feature of centromeric regions across several species. Transcripts have been identified from the central domain of $S$. pombe centromeres as well as from maize centromere sequences $[134,135]$. In human cells, centromeric alpha-satellite repeat transcripts have also been detected in several different cell lines $[136,137]$.

The process of transcription seems to be at odds with the highly stable character of CENP-A containing chromatin, as CENP-A nucleosomes do not appear to turn over except for deposition of newly synthesized CENP-A during G1. However, centromeric character is not mutually exclusive with gene expression. Immunofluorescence studies of 
stretched centromeric chromatin fibers show that centromeres contain H3K4me2, a mark of open or permissive chromatin, and do not contain several H3 modifications implicated in transcription silencing [43]. In addition, human neocentromeres can form in chromosome regions containing actively transcribed genes, and CENP-A nucleosome deposition in gene coding regions of human artificial chromosomes (HACs) does not diminish gene expression $[11,46,138]$. Recent studies demonstrate that modest amounts of transcription across the alpha-satellite centromere region of a HAC are compatible with centromere function; however, driving high levels of transcription does lead to a loss of kinetochore function and a destabilization of CENP-A chromatin, [139] suggesting that the level of transcription may be key to the stability of transcribed centromeres. Recently an accumulation of active RNAPII at human centromeres in metaphase was observed [136]. Analysis of a pseudodicentric chromosome showed that RNAPII only colocalized with the active neocentromere and was not found at the inactivated, alpha-satellite-containing region of the original centromere. Pulse labeling using FITCrUTP showed nascent $\alpha$-satellite transcripts colocalizing specifically with centromeres during mitosis, which were abolished upon $\alpha$-amanitin treatment. Disruption of these centromere transcripts in mitosis caused lagging chromosomes in the subsequent anaphase [136]. The increase in lagging chromosomes correlated with a measurable decrease in centromeric CENP-C levels [136]. CENP-C has been previously implicated as binding to centromeric RNA transcripts suggesting a connection between centromeric transcription and the CCAN [140]. 
While it is clear that centromeric transcription occurs, its function remains unclear. One hypothesis has been proposed where centromeric transcription is coupled to chromatin remodeling activity in order to facilitate the exchange of histone H3 nucleosomes for CENP-A nucleosomes [134]. SSRP1 (structure-specific recognition protein), a subunit of the FACT (facilitates chromatin transcription) complex, was also found to localize to RNAPII foci in human cells [136]. FACT is a general chromatin remodeler that has been found to associate with human CENP-A along with another chromatin remodeler, chromo-helicase DNA-binding protein 1 (Chd1) [20, 21, 141, 142]. The fission yeast homolog, Hrp1, has already been implicated in efficient CENP-A deposition at centromeres [143]. Depletion of Hrp1 caused an increase in H3 nucleosomes in the inner repeats of the fission yeast centromere [134]. Like in humans, the central domains of $S$. pombe centromeres were transcribed by RNAPII to produce small amounts of non-coding RNA (ncRNA) [134]. In both species, Chd1 associates with actively transcribed centromeres and is present at a moment when histone exchange would occur. However, this remains highly speculative and is an area in need of intense study. 


\section{Ensuring CENP-A Centromere Specificity}

The directed recruitment of CENP-A deposition machinery to centromeres is the major method to ensure stable centromere identity. However, overexpression of CENP-A in several systems leads to the misincorporation of CENP-A nucleosomes into other sites within the genome [80-82]. This means that while CENP-A is deposited specifically at centromeres; it retains the ability to localize throughout the rest of the genome. Mislocalization of CENP-A throughout general chromatin by overexpression, or to specific non-centromeric loci via targeted deposition, can cause chromosome missegregation errors $[18,19,81,82]$. Therefore, in order for a cell to ensure the formation of only one centromere per chromosome, it must also employ mechanisms that remove non-centromeric CENP-A. Experimental evidence strongly suggests that this occurs via ubiquitin-mediated proteasome degradation, a mechanism that seems to be conserved from yeast to humans (Figure 1-5).

In both budding yeast and flies, specific E3 ubiquitin ligases have been linked to CENP-A degradation. The S. cerevisiae E3 ligase, Psh1, specifically signals the degradation of Cse4 as compared to histone H3 [144, 145]. Deletion of Psh1 prevents Cse4 from being ubiquitinated and increases the association of $\mathrm{Cse} 4$ at non-centromeric loci $[144,145]$. Psh1 is a major buffer to the effects of Cse4 overexpression, which are lethal in the absence of Psh1 [144, 145]. The major E3 ubiquitin ligase responsible for CenH3/CID degradation in D. melanogaster is the SCF complex component, Ppa [146]. Ubiquitin ligase activity, associated with CENP-A stability, has also been identified in human pathogens. Infection by HSV-1 hijacks the proteasome and targets CENP-A for 
degradation through a viral RING finger domain containing protein, ICP0 [147]. ICP0 is required and sufficient to cause CENP-A degradation after infection [147]. However, a host ubiquitin ligase that is coupled to targeted CENP-A degradation remains to be discovered in humans.

Both Ppa and Psh1 recognize the CATD domain of CENP-A [145, 146]. This is the same region recognized by both the CENP-A chaperone HJURP/Scm3 and the CCAN protein CENP-N in human cells $[65,83,120]$. Consistent with these observations, centromeric pools of $\mathrm{Cse} 4$ in budding yeast are resistant to proteolytic degradation [148]. $\mathrm{Scm} 3$ binding to $\mathrm{Cse} 4$, prevents its ubiquitination by Psh1 in vitro, and turning off Scm 3 expression in vivo accelerates Cse4 degradation in budding yeast [144]. Likewise, depletion of HJURP reduces CENP-A protein levels in human cells [66, 83]. In addition, depletion of Swi/Snf activity in budding yeast causes the accumulation of Cse4 at noncentromeric loci suggesting that non-centromeric CENP-A nucleosomes are sensitive to destabilization by chromatin remodelers [149]. These data suggest that accessibility of CENP-A to degradation is limited by its interaction with either the HJURP chaperone complex or with the CCAN upon incorporation into centromeres. Misincorporated CENP-A lacks these interactions and is therefore removed during remodeling and subjected to proteasomal degradation. 
Figure 1-5. Removal of non-centromeric CENP-A through the proteasome pathway. (A) Centromeric CENP-A is protected from degradation by binding partners that compete with the binding of CENP-A specific, E3 ubiquitin ligases such as Psh1. HJURP binding to unassembled CENP-A/H4 heterodimers or specific interactions between centromeric CENP-A nucleosomes and the CCAN, inhibit the degradation of CENP-A. (B) Non-centromeric CENP-A is removed from ectopic locations in a cell cycle independent manner. This may occur as a natural consequence of histone exchange during chromatin remodeling and transcription across chromosome arms or via a targeted degradation event by a specific E3 ubiquitin ligase, such as Psh1. The CENP-A specific E3 ubiquitin ligase has yet to be found in humans; however, research indicates that CENP-A degradation can occur through the ubiquitin-mediated proteasome pathway in humans. 


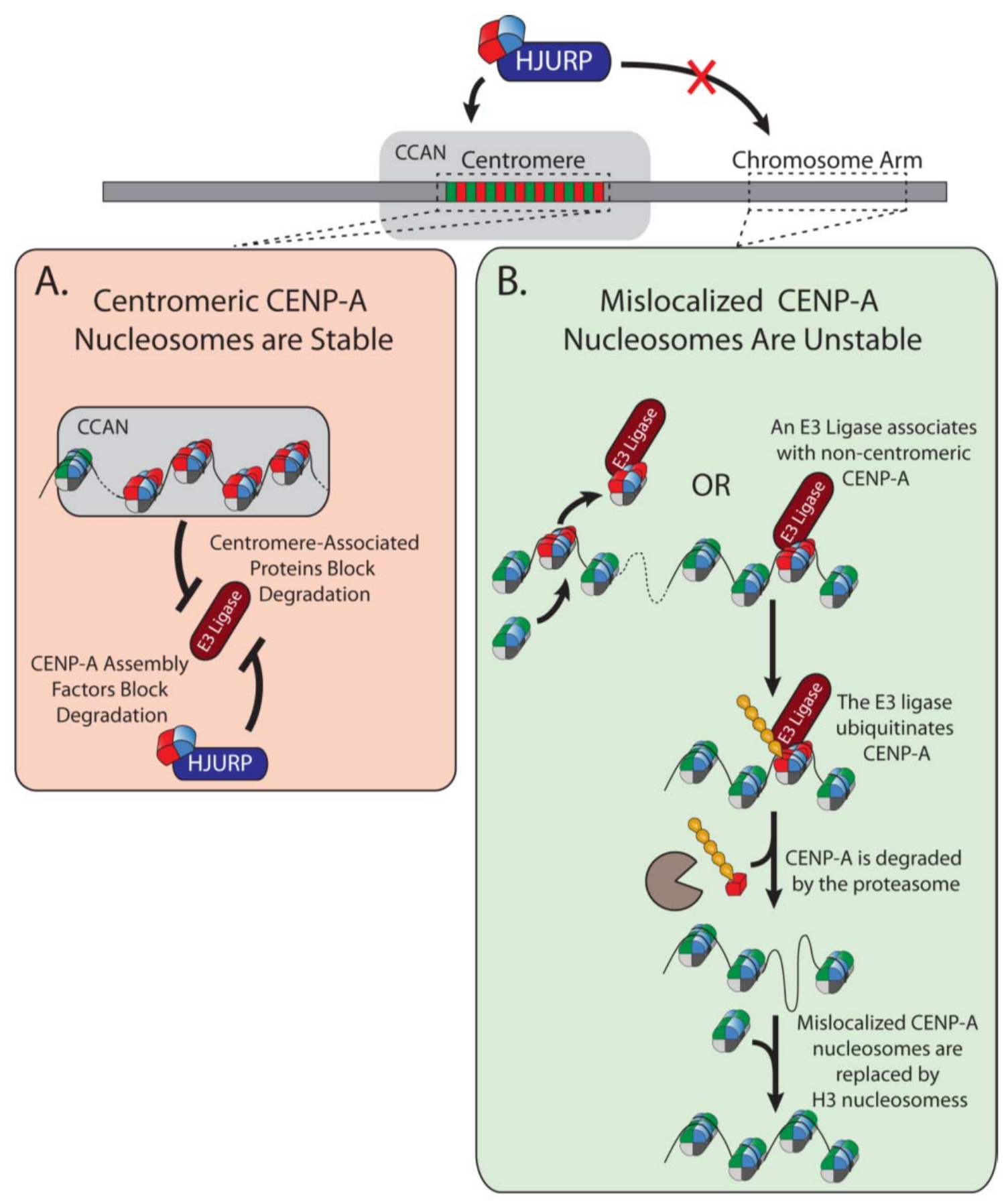

Figure 1-5 


\section{Summary}

The centromere is the chromosomal region that directs kinetochore assembly during mitosis in order to facilitate the faithful segregation of sister chromatids via the mitotic spindle. Centromere location is epigenetically specified by the presence of nucleosomes containing the histone $\mathrm{H} 3$ variant, CENP-A. Maintenance of centromeric identity across cellular generations requires the orchestration of several mechanisms to ensure proper temporal and spatial CENP-A deposition as well as removal of CENP-A from non-centromeric locations. During replication, existing CENP-A nucleosomes are divided among the daughter chromatids to propagate centromere location from one generation to the next. After mitotic exit, new CENP-A nucleosomes are deposited in the correct location every G1 to ensure centromeric CENP-A levels remain constant. Newly deposited CENP-A nucleosomes must then undergo a maturation process to generate stable incorporation into centromeric chromatin. The following chapters highlight several experimental results that elucidate many aspects of the CENP-A deposition pathway; in particular, the role that the Mis 18 complex plays in integrating signals to ensure the proper temporal and spatial regulation of CENP-A deposition at the human centromere. 


\section{Chapter 2 - The Mis18 Complex is Required for Cell Cycle Regulated CENP-A Deposition}

This chapter is based on the following three published works and one manuscript in preparation:

Barnhart, MC, Kuich, PH, Stellfox, ME, Ward, JA, Bassett, EA, Black, BE and Foltz, DR. HJURP is a CENP-A chromatin assembly factor sufficient to form a functional de novo kinetochore. The Journal of cell biology. (2011) 194:229-243.

Silva, MC, Bodor, DL, Stellfox, ME, Martins, NM, Hochegger, H, Foltz, DR and Jansen, LE. Cdk activity couples epigenetic centromere inheritance to cell cycle progression.

Developmental cell. (2012) 22:52-63.

Stellfox, ME, Bailey, AO, and Foltz, DR. Putting CENP-A in its place. Cellular and molecular life sciences: CMLS. (2012).

Nardi, IK., Stellfox, ME, Knippler, CM and Foltz, DR. Liscensing of centromeric chromatin assembly through the Mis18 $\alpha$-Mis $18 \beta$ heterotetramer. In preparation. 


\section{Abstract}

The epigenetic mark that dictates centromere identity is considered to be the presence of nucleosomes containing the histone $\mathrm{H} 3$ variant CENP-A. Human cells deposit newly synthesized CENP-A nucleosomes immediately after mitotic exit, in G1. While centromere architecture varies widely between fission yeast and vertebrates, the deposition of CENP-A is controlled by a group of conserved proteins, which includes the CENP-A chaperone and assembly factor, HJURP, and the Mis 18 complex. The human Mis18 complex consists of Mis18 $\alpha$, Mis18 $\beta$ and Mis18 binding protein 1 (Mis18BP1). Here I will show that the Mis18 complex directly recruits HJURP to centromeric chromatin to initiate CENP-A deposition. In addition, the centromeric localization of the Mis 18 complex is regulated by the direct phosphorylation of Mis $18 \mathrm{BP} 1$ by Cdk1/Cdk2. While all three Mis18 complex proteins are required for CENP-A deposition, Mis18BP1 and Mis $18 \alpha$ bind to the centromere with differing dynamics. Mis $18 \alpha$ remains stably bound to centromeric chromatin in G1 and binds Mis $18 \beta$ via a conserved C-terminal coiled-coil domain. This stable Mis18 $\alpha$-Mis $18 \beta$ complex recruits HJURP in the absence of Mis18BP1 when Mis18 $\alpha$ and Mis18 $\beta$ are artificially targeted to a non-centromeric locus. Therefore, efficient recruitment of HJURP, and downstream CENP-A deposition, requires the presence of Mis $18 \alpha$ and Mis18 $\beta$, whose localization is coupled to cell cycle position by the Cdk1/Cdk2 regulation of Mis $18 \mathrm{BP} 1$. 


\section{Introduction}

During replication, previously incorporated CENP-A containing nucleosomes are distributed to the daughter chromatids. CENP-A protein levels do not rise until near the end of S phase, and the cell proceeds through DNA synthesis, G2 and mitosis with both daughter chromatids having roughly one-half of the centromere's full capacity of CENPA [78, 83-85]. It is only after mitosis that the conserved deposition factors, the Mis 18 complex and HJURP/Scm3, associate with the human centromere and direct the incorporation of the newly synthesized CENP-A nucleosomes into centromeric chromatin $[66,78,83,90,91]$. This indicates that CENP-A deposition is tightly regulated by the cell cycle.

Histone variants have different chaperones and assembly factors to facilitate their incorporation into chromatin [150]. In human cells, HJURP functions as the chaperone and assembly factor for CENP-A containing nucleosomes $[18,66,83]$. HJURP binds to prenucleosomal CENP-A/H4 complexes and prevents the degradation of soluble CENPA. In addition, HJURP is sufficient to assemble CENP-A nucleosomes into chromatin $[18,66,83]$.

Additional factors have been discovered that are required for CENP-A deposition at centromeric chromatin. In fission yeast, a temperature sensitive screen discovered that two proteins, Mis18 and Mis16, are required for CENP-A deposition [91]. Humans and many higher eukaryotes express two Mis18 paralogs, Mis18 $\alpha$ and Mis18 $\beta$ [90]. In addition, human cells also contain a third protein that interacts with Mis18 $\alpha$ and Mis18 $\beta$ at the centromere [90]. This protein, originally discovered in C. elegans as KNL-2, is 
known as Mis18 binding protein 1 (Mis18BP1) in humans [92]. Found in a complex with Mis $18 \alpha$ and Mis $18 \beta$ on chromatin, Mis18BP1 is required for the centromeric localization of Mis18 $\alpha$ and Mis18 $\beta$ and vice versa [90].

Previous work suggests that the Mis18 complex binds to centromeres prior to HJURP localization [83]. A dual staining experiment looked at the localization of Mis18 $\alpha$ and HJURP. A population of G1 cells had only Mis18a localized to centromeres. However, all centromeres that were HJURP positive were also Mis $18 \alpha$ positive [83]. Therefore, this suggests that the Mis18 complex plays an important role in HJURP recruitment.

In the following experiments, I discovered that the Mis 18 complex is responsible for the centromeric recruitment of HJURP, thereby initiating CENP-A assembly in G1. In addition, we showed that CENP-A deposition is cell cycle regulated by $\mathrm{Cdk} 1 / \mathrm{Cdk} 2$ activity. When Cdk kinase activity was high, Mis18BP1 existed in a phosphorylated state and could not associate with centromeric chromatin. After mitotic exit, Cdk activity levels dropped, and Mis18BP1, in a relatively dephosphorylated state, was able to localize to the centromere.

Mis18BP1 freely exchanged with centromeric chromatin during G1, while Mis $18 \alpha$ bound stably to centromeres. Therefore, I assessed which components of the Mis18 complex were specifically required for HJURP recruitment. Artificially targeting Mis $18 \alpha$ to a non-centromeric locus indicated that HJURP only required the presence of Mis18 $\alpha$ and Mis18 $\beta$ once they were recruited to chromatin. Therefore, proper CENP-A deposition was coupled to cell cycle position by $\mathrm{Cdk} 1 / \mathrm{Cdk} 2$ regulation of Mis18BP1 
localization. Once Mis18BP1 was bound to centromeric chromatin, Mis18 $\alpha$ and Mis18 $\beta$ were able to localize and enable HJURP recruitment. 


\section{Results}

\section{Recruitment of HJURP to centromeres requires the Mis18 Complex}

HJURP and the Mis18 complex are both required for the recruitment of CENP-A

to the centromere $[18,66,83,90,91]$. The Mis 18 complex accumulates at centromeres beginning in late anaphase while HJURP localizes to centromeres just after that, during G1 $[83,90]$. While the loading of HJURP seems to follow the Mis18 complex, it is unknown whether HJURP or the Mis 18 complex requires the presence of the other for their centromeric recruitment. In order to shed light on this question, HeLa cells lines stably expressing GFP-tagged Mis $18 \alpha$ or HJURP were generated. GFP-Mis $18 \alpha$ and GFPHJURP localized to centromeres similarly to what would be expected of the wild type proteins. GFP signal was never found at the centromeres of mitotic cells, but was only visible in cells that appeared to be in G1. Then, siRNA was used to deplete protein levels of Mis18 $\alpha$, Mis18BP1 or HJURP in both cell lines.

After 48 hours of treatment with siRNA against Mis18 $\alpha$, GFP-Mis18 $\alpha$ protein levels were reduced to below $25 \%$ of mock treated levels (Figure 2-1A). Analysis of fixed cells using fluorescence microscopy showed that the characteristic centromeric localization of GFP-Mis $18 \alpha$ was also largely abolished after Mis $18 \alpha$ siRNA treatment (Figure 2-1, B and C). Treating cells with siRNA against Mis18BP1 did not significantly lower the protein level of the exogenously expressed GFP-Mis18 $\alpha$, but in agreement with previous data, Mis18BP1 depletion abolished GFP-Mis18 $\alpha$ localization to centromeres (Figure 2-1B) [90]. In contrast, siRNA depletion of HJURP did not significantly alter the localization pattern of GFP-Mis18 $\alpha$ to centromeres. 
In order to determine whether the Mis18 complex affects HJURP recruitment, similar siRNA treatments were performed in the HeLa GFP-HJURP cell line. HJURP siRNA reduced the endogenous and GFP-tagged HJURP to below $12.5 \%$ of normal HJURP protein levels in mock treated HeLa cells (Figure 2-1D). As expected, HJURP depletion completely abolished centromeric localization of the GFP-HJURP protein after 48 hours. While the Mis18 $\alpha$ and Mis18BP1 siRNA treatment did not decrease the protein level of endogenous or exogenous HJURP, the localization of GFP-HJURP to centromeres was decreased to $0.0 \%$ and $0.3 \%$ respectively in randomly cycling cells (Figure 2-1, E and F). These data suggest that while HJURP is not required for the localization of the Mis18 complex to centromeres, in the absence of the Mis18 complex cells fail to recruit HJURP to centromeric chromatin. 
Figure 2-1. Recruitment of HJURP to centromeres requires the Mis18 complex.

(A) Anti-GFP western blot of HeLa GFP-Mis $18 \alpha$ lysates. Each lane contains lysate from approximately $1 \times 10^{5}$ cells. The HeLa control lysates are from HeLa cells not expressing GFP-Mis18a. (B) Representative maximum projections of deconvolved z-stacks in preextracted, fixed HeLa GFP-Mis18 $\alpha$ cells. All images were procured with the same exposure time and scaled equally in the CENPT (red) and GFP-Mis18 $\alpha$ (green) channels. DAPI staining was overlaid onto the DIC image with arrows to highlight midbodies. Scale bar $=5 \mu \mathrm{m}$. (C) Average percentage of GFP-Mis $18 \alpha$ positive nuclei (see Materials and Methods). Error bars show the standard deviation between replicates. (D) AntiHJURP western blot of HeLa GFP-HJURP lysates treated similarly as in A. The standard curve was generated with plain, untreated HeLa cell lysates. (E) Similar image acquisition as in C in the HeLa GFP-HJURP cell line. (F) Average percentages of GFPHJURP positive nuclei in each condition. Error bars show the standard deviation between replicates. 

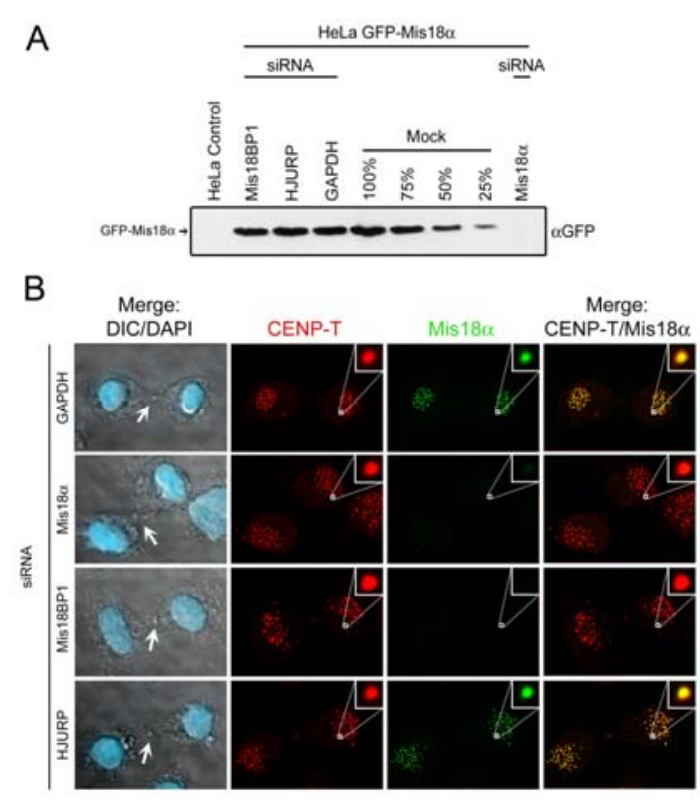

C
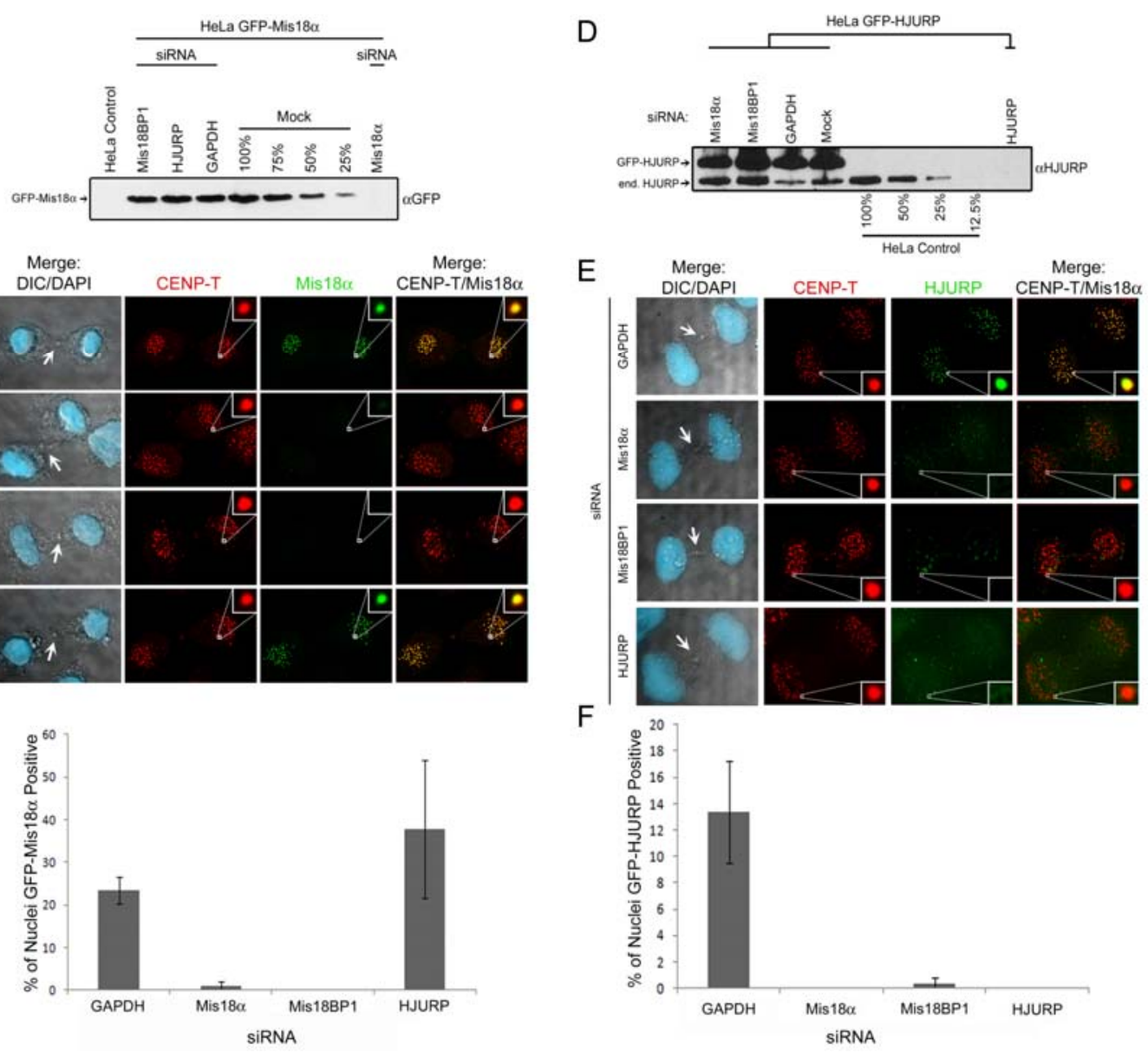

$\mathrm{F}$

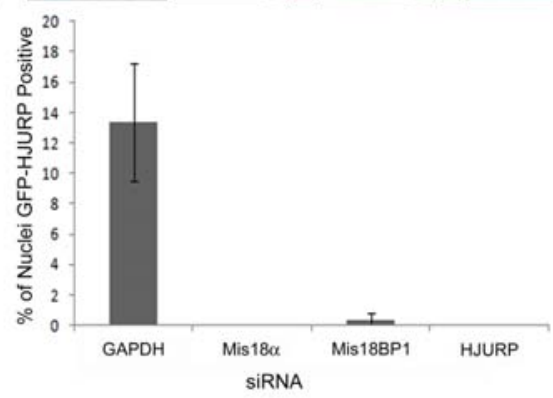

Figure 2-1

Published as Figure 6 in Barnhart, M.C., et al. (2011). The Journal of Cell Biology. 


\section{The Mis18 complex regulates the cell cycle timing of CENP-A}

\section{deposition}

In human cells, CENP-A deposition is uncoupled from the incorporation of canonical histones during S phase and only occurs in G1 of the following cell cycle [84]. Therefore a cell must activate CENP-A deposition during the proper time as well as prevent CENP-A deposition during other phases of the cell cycle. As shown above, the Mis18 complex is required for the CENP-A assembly factor, HJURP, to localize to centromeres. Those results and previous work indicate that the localization of the Mis 18 complex to centromeric chromatin is arguably the first step in the CENP-A deposition pathway $[83,90,91]$.

The Mis18 complex localizes to centromeres in late anaphase, directly after mitotic exit. Cell cycle progression is mediated by several kinases that regulate the passage of a cell from one state to another, which indicates a possible regulatory mechanism for Mis18 complex recruitment by cell cycle kinases [98]. As cells exit mitosis, mitotic kinase activity drops dramatically. To assess changes to the Mis18 proteins during mitosis, prior to their localization to the centromere, I isolated whole cell lysates from randomly cycling cells and compared them to lysates from cells blocked in nocodazole for 12 hours. Western blot analysis of the lysates showed that the levels of phosphorylated serine 10 of histone $\mathrm{H} 3(\mathrm{H} 3 \mathrm{~S} 10 \mathrm{p})$ increased in the nocodazole treated cells as compared to the randomly cycling cell population (Figure 2-2A). Randomly cycling cells would be expected to have roughly $10 \%$ of the whole cellular population in mitosis at any one time. Therefore, the increase in levels of H3S10p signal in the 
nocodazole treated cell lysates indicated that I had successfully made a mitotically arrested population. Blotting for endogenous Mis18BP1 identified a mitotic form of Mis18BP1 that traveled through a SDS-PAGE gel with a slightly larger apparent molecular weight than in the randomly cycling lysates (Figure 2-2A). Mitosis is a hyperphosphorylated state with respect to mitotic kinase activity, and this shift in apparent molecular weight suggested a mitotic phosphorylation event on Mis18BP1.

In order to confirm that the increase in apparent molecular weight of Mis 18BP1 in mitosis was caused by a change in phosphorylation state, I developed a pull down and calf intestinal alkaline phosphatase (CIP) treatment assay (Figure 2-2B). Cells stably expressing GFP-tagged Mis18BP1 were blocked in mitosis as in Figure 2-2A. Whole cell lysates from randomly cycling and mitotic populations were run over Ni-NTA agarose column that was coated with His-tagged GFP-binding protein (His-GBP). Bound proteins were left untreated or treated with CIP in the absence or presence of an excessive concentration of the phosphatase inhibitor, sodium orthrovanadate $(\mathrm{NaV})$.

Two bands are present in each GBP pull downs presumably because GFPMis18BP1 constitutively interacted with endogenous Mis18BP1. GBP bound fractions from untreated mitotic lysates only contained the higher molecular weight bands for GFP-Mis 18BP1 and the endogenous protein. However, those cells, which had been blocked in mitosis, but were also treated with CIP for 1 hour only contained lower molecular weight bands, similar to the bands present in GBP pull down from randomly cycling cells. When CIP activity was blocked by sodium orthovanadate, the upper 
molecular weight bands remained in the $\mathrm{CIP}+\mathrm{NaV}$ pull downs. Therefore, the molecular weight shift in Mis18BP1 is the result of direct phosphorylation of Mis18BP1 in mitosis.

In order to further confirm this conclusion, we sought to generate a Mis18BP1 construct that can no longer be phosphorylated in mitosis. Such a construct would no longer display the upward molecular weight shift. Our collaborators predicted 24 possible phosphorylation sites on Mis18BP1 (Figure 2-2C). Many of these sites were Cdk consensus sites. Cdk1 and Cdk2 activities peak in mitosis, and there were ideal candidate kinases for mitosis-specific phosphorylation of Mis18BP1 [98]. Our collaborators mutated all 24 putative phosphorylation sites to alanine and generated an N-terminal GFP fusion protein (GFP-Mis18BP1 $\left.1^{24 \mathrm{~A}}\right)$.

Expressing this construct in cells allowed us to assess the presence or absence of the molecular weight shift in mitotically arrested cell lysates as in Figure 2-2A. Wild type GFP-Mis18BP1 (GFP-Mis18BP1 ${ }^{\mathrm{wt}}$ ) transfected cells that were allowed to randomly cycle had two prominent bands when blotted with an antibody against Mis18BP1. The lower band corresponded with the endogenous protein, and the higher molecular weight band corresponded to the wild type GFP-tagged construct (Figure 2-2D, left panel). When these cells were blocked in mitosis with nocodazole overnight, four Mis18BP1 bands appeared. The two upper bands corresponded to GFP-Mis18BP $1^{\mathrm{wt}}$ protein, and the lower two bands corresponded to the two forms of endogenous Mis18BP1. However, in cells that were transfected with the GFP-Mis18BP1 ${ }^{24 \mathrm{~A}}$ construct, the highest molecular weight band, which would correspond to the phosphorylated form of GFP-Mis $18 \mathrm{BP} 1^{24 \mathrm{~A}}$, was not seen in the mitotically arrested lysates (Figure 2-2D, right panel). While the 
multiple alanine mutants do not indicate which phosphorylation sites contributed to the mitotic molecular weight shift, it is clear that this mutant prevents mitotic phosphorylation events on Mis18BP1.

In order to delineate the function of the cell cycle specific phosphorylation of Mis18BP1, our collaborators in the Jansen laboratory performed a series of in vivo experiments that clearly indicated that Mis18BP1 phosphorylation regulates the cell cycle timing of CENP-A deposition. Their first step was to see if this mitotic phosphorylation played a role in regulating the cell cycle specific, centromeric recruitment of the Mis18 complex. GFP-Mis18BP $1^{\text {wt }}$ and GFP-Mis $18 \mathrm{BP} 1^{24 \mathrm{~A}}$ were transiently transfected into cells, and the cellular localization of was assessed. CENP-T staining marked endogenous centromeres and high levels of cyclin B staining indicated non-G1 cells. In the cells transfected with GFP-Mis18BP1 ${ }^{\mathrm{wt}}$, GFP signal was not seen at centromeric foci in $\mathrm{G} 2$ and mitotic cells (Figure 2-2E, left panels). This follows the normal localization pattern previously described [90]. In addition the centromeric GFP intensity of the mitotic and G2 populations transfected with GFP-Mis $18 \mathrm{BP} 1^{\text {wt }}$ was low (Figure 2-2F). Interestingly, G2 and mitotic cells transfected with GFP-Mis18BP1 ${ }^{24 \mathrm{~A}}$ clearly had GFP signal at centromeres (Figure 2-2E, right panels). In addition, the centromeric GFP intensity of these two populations was higher than in the wild type GFP-Mis18BP1 transfection condition (Figure 2-2F). Therefore, the ability of a cell to phosphorylate Mis18BP1 in G2 and mitosis is required to keep the Mis 18 complex off of centromeric chromatin, outside of G1. 
However, the question remained as to whether this regulation also affected downstream CENP-A deposition. If Mis18BP1 was able to associate with centromeres outside of its normal G1 time frame, could it also recruit HJURP and begin the CENP-A deposition pathway during other cell cycle phases? In order to answer this question, our collaborators used a SNAP-tag assay. The SNAP tag is a protein tag that is classified as a "suicide enzyme". It catalyzes a covalent linkage between a substrate and itself, which then inactivates the enzyme.

Cells stably expressing a SNAP-tagged CENP-A were synchronized with a double thymidine block to the G1/S boundary. Cells were treated or "quenched" with a non-fluorescent substrate of the SNAP-tag (BTP). This effectively labeled the previously translated pool of CENP-A-SNAP with a non-fluorescent tag. Cells were then released from the thymidine block and the BTP was washed out. This allowed cells to progress through S-phase and synthesize new, unlabeled CENP-A-SNAP protein. Seven hours after release from the thymidine block, the cells were simultaneously treated with roscovitine, a Cdk1/Cdk2 inhibitor and the fluorescent SNAP substrate (TMR-Star). Only the CENP-A molecules, synthesized after release from the thymidine block, would be fluorescently labeled with TMR-Star. In G2 cells not treated with roscovitine (G2 control), GFP-Mis18 $\alpha$ was not localized to centromeres and no TMR-Star-labeled CENPA-SNAP was recruited to centromeric chromatin (Figure 2-2, G and H). However in G2 cells treated with roscovitine, a large percentage of cells were not only positive for GFPMis18 $\alpha$ at endogenous centromeres, but also had centromeric TMR-Star-labeled CENPA-SNAP present. Therefore, when $\mathrm{G} 2$ cells were treated with a Cdk1/Cdk2 inhibitor, the 
Mis18 complex was no longer inhibited by phosphorylation. Without this inhibition, the Mis 18 complex was free to associate with the centromere and initiate the CENP-A deposition pathway, outside of G1. 
Figure 2-2. The Mis18 complex regulates the cell cycle timing of CENP-A deposition.

(A) Western blots against H3S10p and endogenous Mis18BP1 of whole cell lysates from randomly cycling (nocodazole -) and mitotically arrested (nocodazole + ) populations. (B) Anti-Mis18BP1 western blots of GBP bound GFP-Mis18BP1 from randomly cycling and mitotically arrested cell lysates. NT represents mitotic lysates that received no further treatment after nocodazole arrest; CIP indicates lysates that were incubated at $37^{\circ} \mathrm{C}$ for 1 h with 60 units of CIP, CIP $+\mathrm{NaV}$ represents lysates that were incubated with CIP in the presence of $10 \mathrm{mM}$ sodium orthovanadate for $1 \mathrm{~h}$ at $37^{\circ} \mathrm{C}$. (C) Diagram depicting the putative Cdk sites and other phosphorylation sites on Mis18BP1. (D) Anti-GFP and antiMis18BP1 western blots of whole cell extracts from HeLa T-Rex cells transfected with either GFP-Mis18BP $1^{\text {wt }}$ or GFP-Mis $18 \mathrm{BP} 1^{24 \mathrm{~A}}$. (E) Representative images of GFPMis $18 \mathrm{BP} 1^{\mathrm{wt}}$ and GFP-Mis18BP1 ${ }^{24 \mathrm{~A}}$ transfected into HeLa cells and stained for cyclin B1 and CENP-T. (F) Box and whisker plots of centromeric GFP intensity of GFPMis $18 \mathrm{BP} 1^{\mathrm{wt}}$ and GFP-Mis18BP1 ${ }^{24 \mathrm{~A}}$ in $\mathrm{G} 2$ and mitosis. $(\mathrm{G})$ Diagram depicts the cell synchronization, SNAP-tag protocol and inhibitor treatment of CENP-A-SNAP expressing cells. Images depict the GFP-Mis $18 \alpha$ localization and CENP-A-SNAP recruitment in G2 cells with and without roscovitine treatment. (H) Quantification of G1 and $\mathrm{G} 2$ cell populations from the experiment described in G. 
A

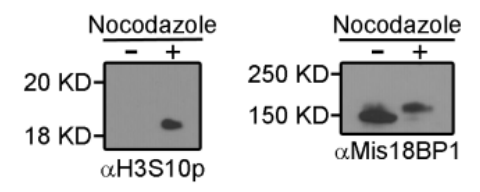

B

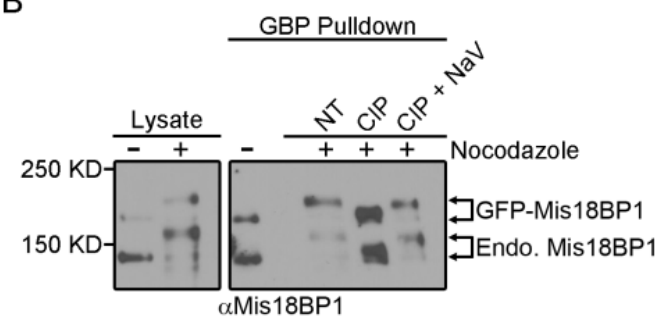

C

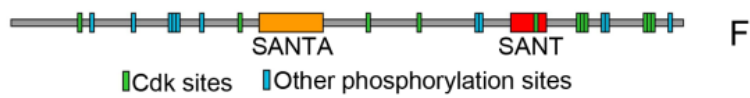

D

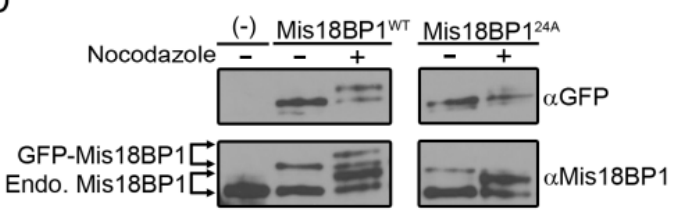

E

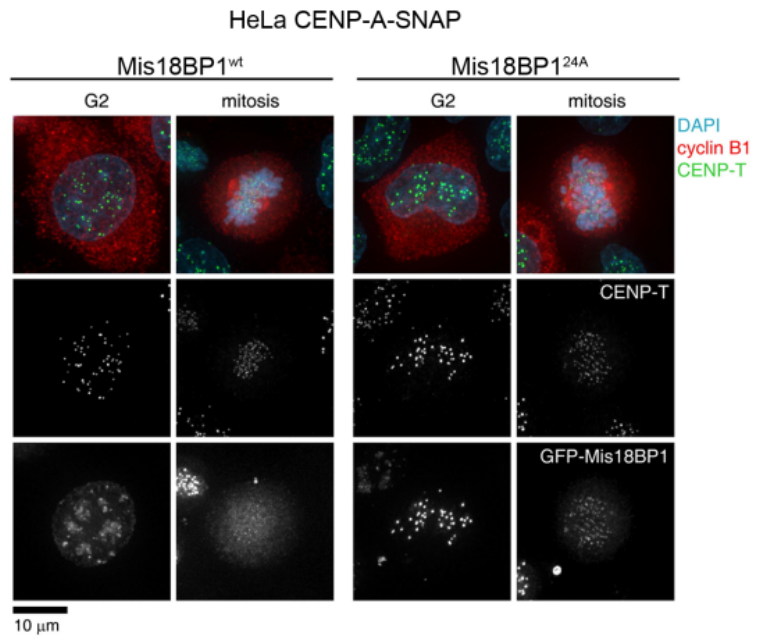

$\mathrm{F}$

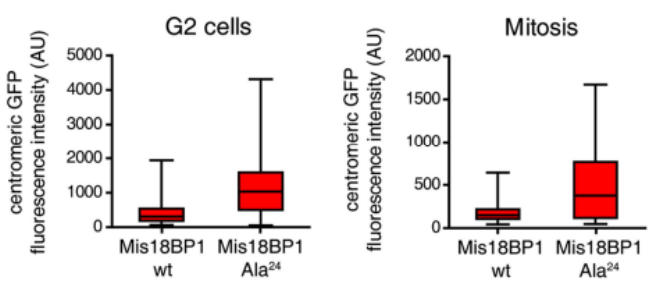

G

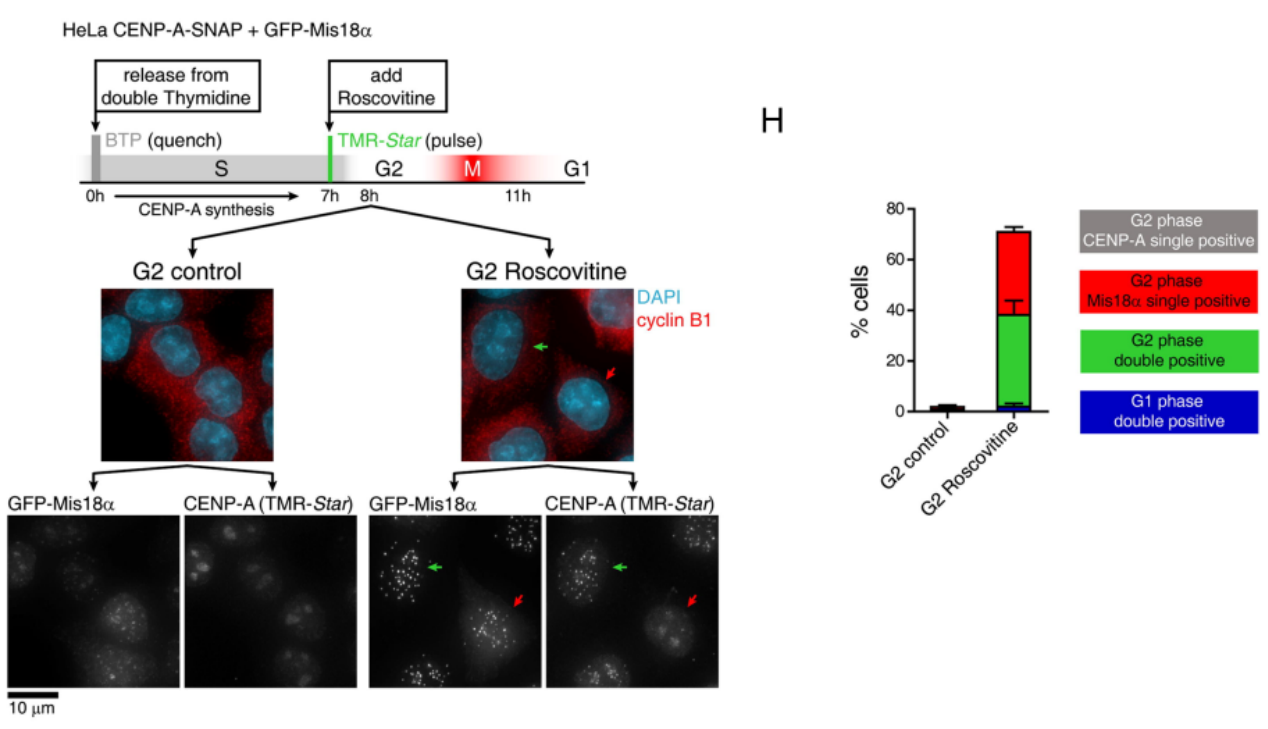

Figure 2-2

Published as Figure 4 and Figure 5 in Silva, M.C., et al. (2013) Developmental Cell.

Data in panel C and E-H was generated in collaboration with the Jansen Laboratory 


\section{Mis18 $\alpha$ and Mis18BP1 bind centromeric chromatin with differing}

\section{dynamics}

The Mis 18 complex associates with centromeric chromatin directly after mitotic

exit, and I have shown that its presence in the cell is required for HJURP recruitment and subsequent CENP-A deposition. Previous work has highlighted the possibility that the Mis 18 complex might license centromeric chromatin and make it available for CENP-A deposition in G1 [90, 109]. Since there are several unique epigenetic aspects of centromeric chromatin, the hypothesis that a complex required for CENP-A deposition may mediate a change in the epigenetic character of centromeric chromatin remains an interesting hypothesis.

However, it is known that members of the Mis 18 complex can interact with proteins of the CCAN, which will be discussed further in chapter 3. However, the dynamics of how the Mis18 complex interacts with centromeric chromatin in humans is currently unknown. In an enzymatic process, one would expect the Mis18 complex to bind to its substrate, modify it and move on to the next substrate site. Following substrate modification by the Mis 18 complex, HJURP would then recognize the modified nucleosome as the site for proper, regulated CENP-A deposition. This type of interaction with centromeric chromatin would require that the Mis 18 complex have a rapid rate of turnover and a minimal retention time during each enzyme cycle during G1.

In contrast, the Mis 18 complex might serve as an adapter. The complex may contain a recognition motif that recognizes a certain aspect of centromeric chromatin and stably binds to that unique characteristic. The Mis 18 complex would then serve as a 
platform for additional downstream effectors of CENP-A deposition. Such a mechanism would require a more stable interaction between the Mis18 complex and the centromere during G1, with little exchange as CENP-A deposition occurs.

In order to study the interaction of the Mis18 complex with centromeric chromatin I used a fluorescence recovery after photobleaching (FRAP) assay to determine the binding dynamics of the Mis18 complex in G1. Overexpression of GFPMis18 $\alpha$ and GFP-Mis18BP1 in HeLa cells allowed us to assess the dynamics of the G1 centromeric localization of the Mis18 complex. For the assay, I chose cells that had a focal, GFP localization pattern and cell morphology to suggest G1 placement in the cell cycle. Individual centromeres were then bleached and the fluorescence recovery was measured over the following 4.5 minutes (Figure 2-3).

While all three proteins of the Mis 18 complex are mutually required for localization to centromeres and for CENP-A deposition, FRAP analysis of GFP-Mis18 $\alpha$ and GFP-Mis18BP1 indicated that individual components of the Mis 18 complex bind to centromeric chromatin with very different dynamics. GFP-Mis18BP1 showed clear recovery of fluorescence over time. Recovery curve analysis showed that the $t_{1 / 2}$ was $44.3 \mathrm{~s}$ and the final recovery was $68.2 \%$. This indicated a steady recovery to more than half of the original intensity. This dynamic profile argued that Mis18BP1 was continually turned over at centromeric chromatin during G1.

On the other hand, GFP-Mis18 $\alpha$ had a much less dynamic interaction profile. After photobleaching of GFP-Mis $18 \alpha$ positive centromeres, the GFP signal recovered to only $19.9 \%$ of the initial, pre-bleach intensity. This data suggested that during G1 Mis $18 \alpha$ 
loads onto the centromere and remains stably associated. While all Mis 18 proteins associate with centromeric chromatin at the same point in the cell cycle and require each other's presence, they interact with the centromere via very different mechanisms. This suggests that individual components of the Mis 18 complex may serve separate functions in the downstream CENP-A deposition pathway. 
Figure 2-3. Mis18 $\alpha$ and Mis18BP1 bind centromeric chromatin with differing dynamics.

(A) Representative images of fluorescence recovery after photobleaching (FRAP) of GFP-Mis 18BP1 (top) and GFP-Mis18 $\alpha$ (bottom). Upper insets follow unbleached, control centromeres, while lower insets highlight an individual photobleached centromere. (B) A fluorescence recovery plot of photobleached centromeres over time. Closed circles indicate the average recovery of GFP-Mis18BP1 and open circles indicate the recovery of GFP-Mis18 1 . Both plots were fitted to a single exponent recovery curve $\mathrm{A}^{*}\left(1-e^{-\mathrm{kt}}\right)$ using 'nls' function in R. Table above the graph describes the total recovery, $t_{1 / 2}$, and the $\mathrm{R}^{2}$ value determined by the fitted recovery curve. 
A

GFP-Mis18BP1

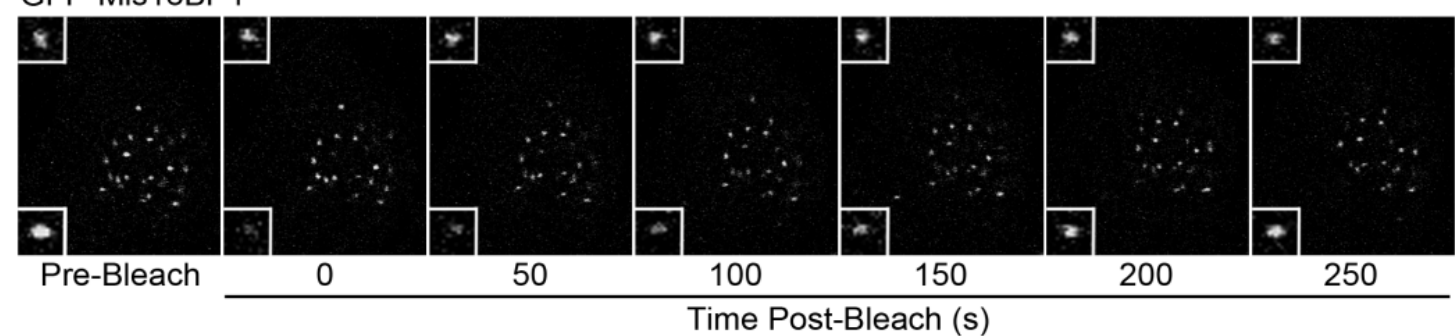

GFP-Mis18 $\alpha$

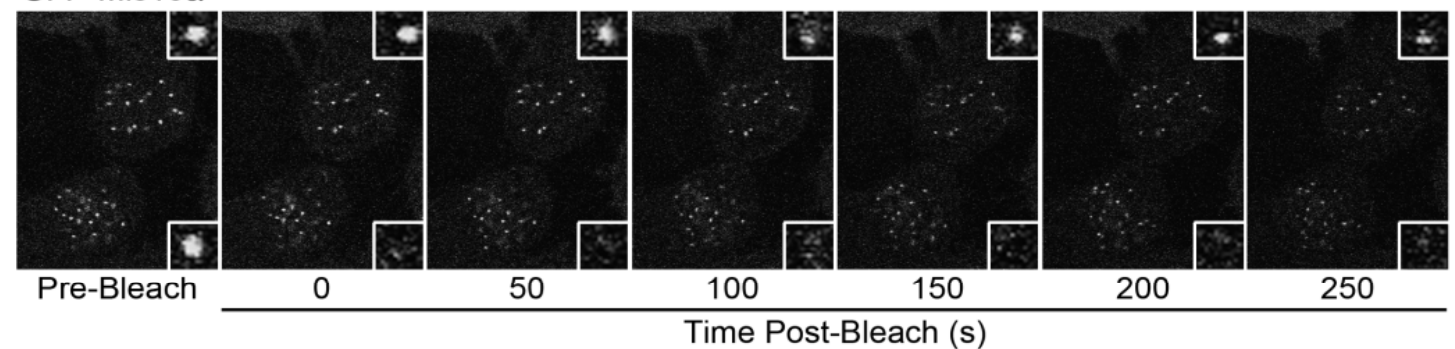

B

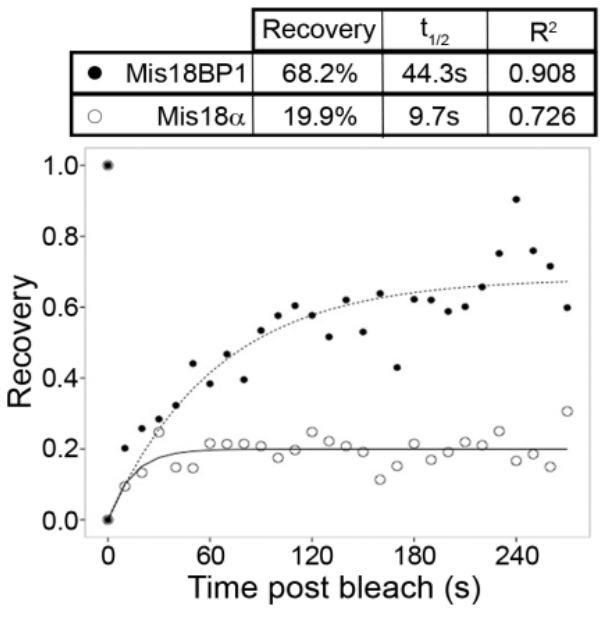

Figure 2-3 


\section{Mis18 $\alpha$ and Mis18 $\beta$ interact through conserved C-terminal coiled-coils}

The data generated by FRAP analysis indicated that Mis $18 \alpha$ is stably bound to centromeres during G1. Mis18 $\alpha$ and Mis $18 \beta$ protein levels are tightly correlated and the depletion of one paralog results in a decreased protein level of the other [90]. Since both proteins are required for the localization of HJURP to centromeres and subsequent CENP-A deposition, a better understanding of the physical interactions between Mis18 $\alpha$ and Mis $18 \beta$ would increase our knowledge of how this protein complex directs CENP-A incorporation during G1 [151].

I ran the protein sequences of human Mis18 $\alpha$ and Mis18 $\beta$ through the Paircoils2 coiled-coil prediction program and graphed the p-value versus the amino acid number (Figure 2-4A) [152]. Both Mis18 $\alpha$ and Mis18 $\beta$ contained a C-terminal coiled-coil with a predicted p-value of less than 0.03 (Figure 2-4A, dotted line). Mis18 $\alpha$ also had a second, N-terminal coiled-coil. This N-terminal coiled-coil had a slightly lower probability score, but the p-value was still less than 0.05. In Figure 2-4B, I diagramed the amino acid positions of a canonical, parallel coiled-coil using a helical wheel model adapted from Mason, et al 2006 [153]. The fuchsia positions (a and d) correspond to the hydrophobic residues that lie in the central domain of the coiled-coil, while the green positions (e and g) correspond to the ionic interactions that help maintain the interaction surface.

Clustal2W alignments generated by Geneious, displayed the conservation of amino acid residues within the predicted coiled-coil region from several evolutionarily diverse organisms (Figure 2-4C) [154]. Above the alignment, the coiled-coil position markers assigned by the Paircoil2 program for human Mis18 $\alpha$ and Mis18 $\beta$ were 
indicated. These letters corresponded to the position of each amino acid in the predicted coiled-coil, diagramed in Figure 2-4B. The level of conservation suggested that this protein-protein interaction motif is likely a highly conserved method of Mis 18 complex formation.

In order to assess the role the coiled-coils play on the function of the Mis18 complex, I expressed GFP-tagged full-length and coiled-coil deletion constructs of Mis $18 \alpha$ and Mis18 $\beta$ in U2OS cells and assessed the constructs' ability to localize to endogenous centromeres (Figure 2-4D - H). Both full-length and coiled-coil deletion constructs of Mis18 $\alpha$ and Mis18 $\beta$ were expressed at similar levels, which indicated that deleting the C-terminal portion of either protein did not dramatically alter the GFP construct's stability (Figure 2-4E). Both full-length GFP-Mis18 $\alpha$ and GFP-Mis $18 \beta$ localized to centromeres in a proportion of randomly cycling cells, which was expected due to the known G1 localization pattern of both proteins (Figure 2-4F). For unknown reasons, full-length GFP-Mis18 $\alpha$ localized to centromeres in a higher proportion of cells $(13.0 \pm 4.2 \%)$ than the full-length GFP-Mis $18 \beta$ construct $(5.9 \pm 0.1 \%)$. However, both results indicated that the localization of either protein maintained a regulated pattern of centromeric recruitment. In contrast, centromeric localization of both Mis18 $\alpha$ and Mis1 $8 \beta$ coiled-coil deletion mutants was abolished. Neither GFP-Mis $18 \alpha^{2-175}$ nor GFPMis $18 \beta^{2-188}$ was found to colocalize with endogenous CENP-T foci.

Two hypotheses arose from this observation. Either the coiled-coils were part of a required domain for Mis18 $\alpha$ and Mis18 $\beta$ to recognize the centromere, or the coiled-coils were required for the two proteins to interact with each other physically. Previous studies 
documented that Mis $18 \alpha$ and Mis $18 \beta$ protein stability and centromeric localization are mutually dependent on each other [90]. Therefore, if deleting the coiled-coil regions of Mis $18 \alpha$ and Mis18 $\beta$ prohibit the physical interaction between the proteins, then they would also be unlikely to localize properly.

In order to separate Mis $18 \alpha$-Mis18 $\beta$ binding from centromeric localization, I used a cell line with an incorporated LacO array on chromosome 1 to artificially target Mis18 $\alpha$ to chromatin and assess the ability of Mis $18 \beta$ to interact with Mis18 $\alpha$. Mis $18 \alpha$ constructs were fused to mCherry-LacI in order to drive their recruitment to the integrated LacO array (Figure 2-4G). Cells were then cotransfected with the mCherry-LacI-Mis18 $\alpha$ constructs as well as full-length GFP-Mis18 $\beta$, and mCherry-LacI alone was used as a negative control. Transfected cells were fixed and stained, and the ability for the various mCherry-LacI constructs to recruit GFP-Mis $18 \beta$ to the array was assessed by fluorescent microscopy (Figure 2-2, G and H).

Almost $100 \%$ of cells that expressed full-length mCherry-LacI-Mis $18 \alpha$ recruited GFP-Mis $18 \beta$ to the array. In addition, deletion of the putative coiled-coil near the Nterminus of Mis $18 \alpha$ (mCherry-LacI-Mis $18 \alpha^{81-233}$ ) was also able to recruit GFP-Mis18 $\beta$ with a level of efficiency similar to wild type Mis18 $\alpha$. However, when a Mis18 $\alpha$ construct that lacked the C-terminal coiled-coil (mCherry-LacI-Mis $18 \alpha^{2-175}$ ) was targeted to the LacO array, GFP-Mis18 $\beta$ was no longer recruited. Therefore, Mis18 $\alpha$ and Mis $18 \beta$ required the C-terminal coiled-coil domain to interact in vivo. 
Figure 2-4. Mis18 $\alpha$ and Mis18 $\beta$ interact through conserved C-terminal coiled-coils.

(A) Graphs of the p-value calculated by Paircoil2 versus amino acid position of human Mis18 $\alpha$ and Mis18 $\beta$ [152]. (B) Helical wheel diagram, modified from Mason et al., 2006, showing the physical positioning and the interactions between hydrophobic (magenta) and ionic (green) residues in a canonical parallel coiled-coil. Diagram depicts the structure looking down the coil axis. (C) Clustal2W alignment of Mis18 homologs across evolutionarily divergent species generated by Geneious software [154]. Grayscale highlights amino acid conservation. Color-coded letters above indicate the predicted position in the coiled-coil of the human amino acid sequence. Colors correspond to the helical wheel diagram in B. (D) Schematic of putative coiled-coil regions (grey) in human Mis18 $\alpha$ and Mis18$\beta$. (E) Anti-GFP western blot showing the expression GFPtagged wild type and coiled-coil deletion constructs of Mis18 $\alpha$ and Mis18 $\beta$ in U2OS cells. (F) Representative images of U2OS cells transfected as in E. CENP-T staining marks endogenous centromeres. White boxes and enlarged images highlight GFP and CENP-T colocalization at individual centromeres. Numbers in white indicate the average percentage of randomly cycling, transfected cells that have centromeric GFP signal, plus or minus the standard deviation between replicates. Scale bars $=5 \mu \mathrm{m}$. (G) Representative images of mCherry-LacI constructs targeted to the $\mathrm{LacO}$ array and the recruitment of GFP-Mis18 $\beta$. CENP-T staining marks endogenous centromeres. White boxes and enlarged images highlight mCherry-LacI/GFP colocalization at the LacO array. Scale bars $=5 \mu \mathrm{m}$. $(\mathrm{H})$ Graph depicting the percentage of transfected cells that recruited full-length GFP-Mis $18 \beta$ to the array. 
A

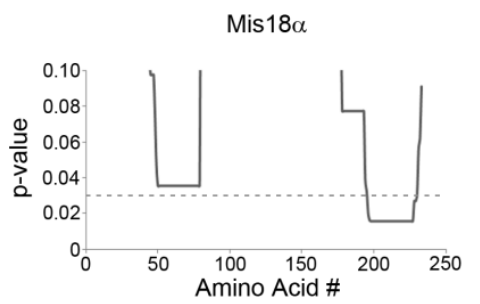

Mis18 $\beta$

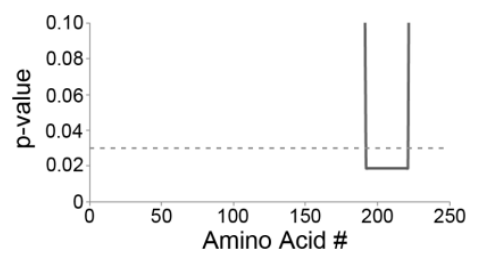

B

C

Mis18 $\alpha$

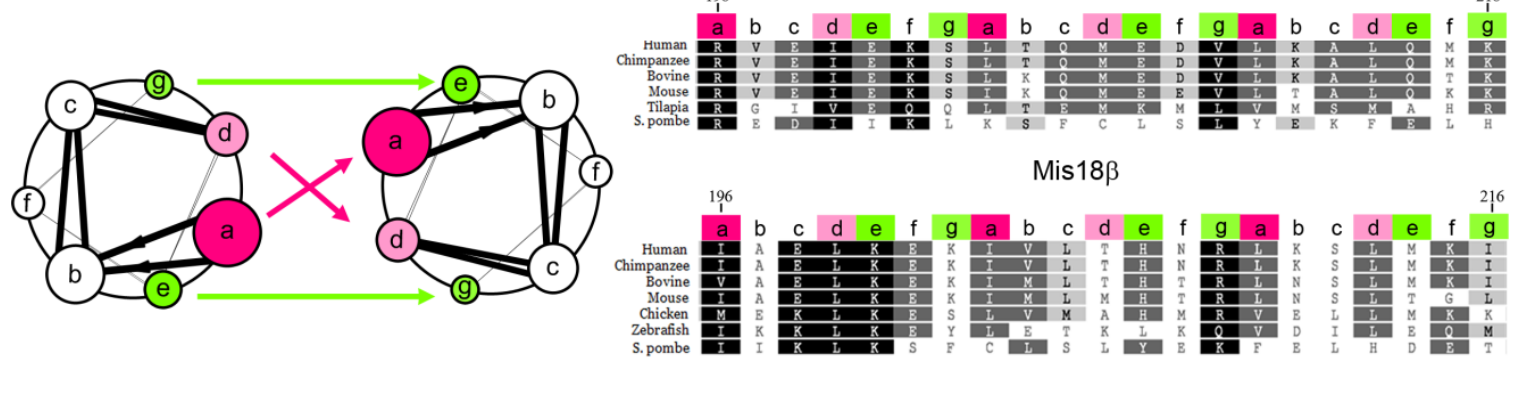

D

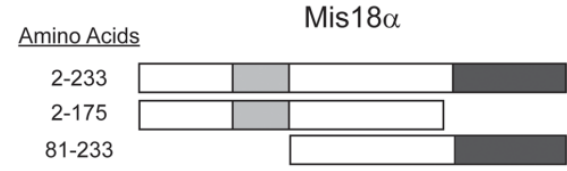

Amino Acids

2-229

2-188

E

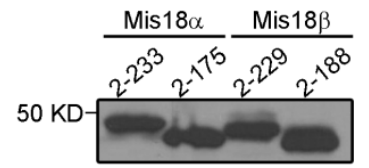

F

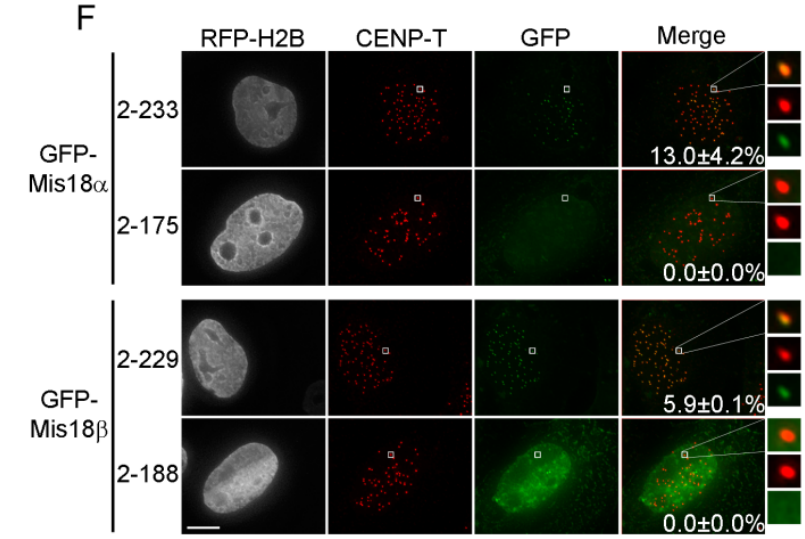

G

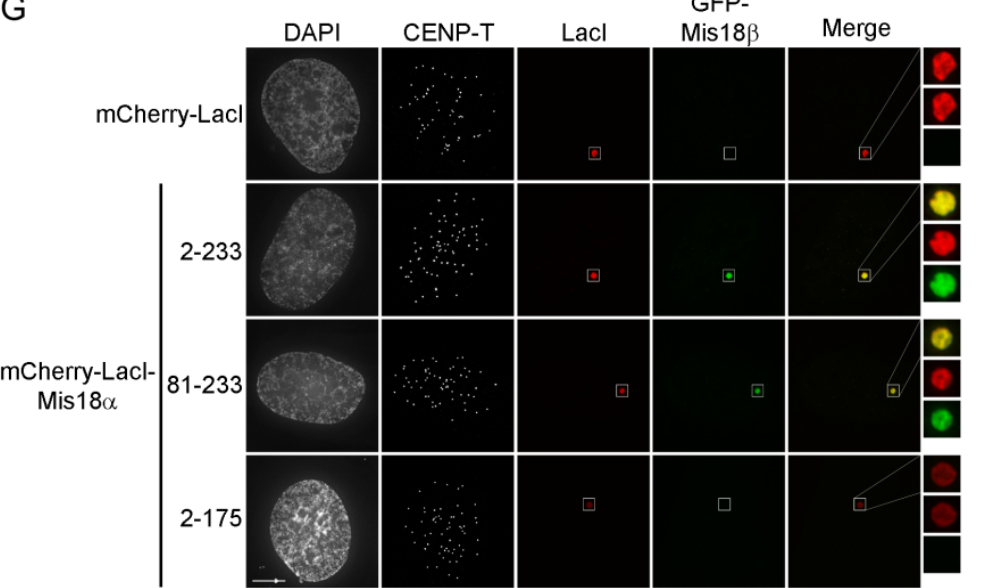

$\mathrm{H}$

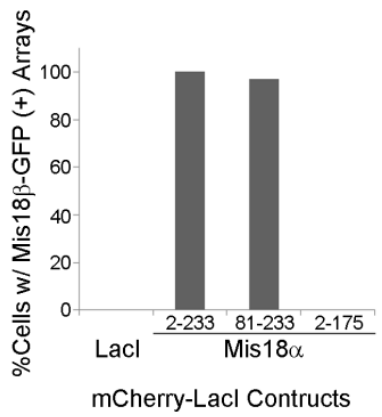

Figure 2-4 


\section{Mis18 $\alpha$ and Mis18ß recruit HJURP to centromeres for CENP-A}

\section{deposition}

It is known in human cells that all three Mis18 proteins are required for HJURP recruitment to centromeres and the downstream incorporation of newly synthesized CENP-A nucleosomes into centromeric chromatin $[18,155]$. FRAP experiments discussed above indicate that the Mis18 complex proteins may serve different functions during CENP-A deposition. While all three proteins are required for the localization of the complex, it has not been determined which components of the Mis 18 complex are specifically required for HJURP recruitment.

In order to assess which components of the Mis 18 complex, uncoupled from their mutual dependency for centromeric localization, specifically recruited HJURP, I once again used the LacO array system. I targeted mCherry-LacI-Mis18 $\alpha$ to the array and cotransfected in GFP-Mis $18 \beta$ and HA-tagged full-length HJURP (HA-HJURP). By cotreating with control siRNA and siRNA that targeted endogenous Mis18BP1, I was able to assess HJURP recruitment to chromatin by Mis $18 \alpha$ and Mis $18 \beta$ in the presence and absence of endogenous Mis18BP1.

In the Mis18BP1 siRNA treated cells, protein levels of Mis18BP1 decreased to less than $10 \%$ of the levels in GAPDH control siRNA treated cells (Figure 2-5A). In the presence of endogenous Mis18BP1, HJURP was recruited to the LacO array in $67.6 \pm$ 9.7\% of triply transfected cells (Figure 2-5, B and C). Despite the robust reduction in Mis18BP1 protein levels, HJURP was recruited to the mCherry-LacI-Mis18 $\alpha$ array in $89.2 \pm 0.3 \%$ of triply transfected cells treated with Mis18BP1 siRNA. 
In addition, I assessed whether the conserved coiled-coils were required for HJURP recruitment to the array. I targeted mCherry-LacI-Mis18 $\alpha$ to the array and assessed whether the coiled-coil deletion mutant would be able to recruit HJURP without its interaction with Mis18 $\beta$. When full-length mCherry-LacI-Mis18 $\alpha$ was targeted to the array, GFP-Mis18 $\beta$ and HA-HJURP were both robustly recruited to the LacO array as expected based on our previous experiments. However, when we targeted the coiled-coil deletion mutant of Mis $18 \alpha$ (mCherry-LacI-Mis $18 \alpha^{2-175}$ ) to the array, HJURP was no longer recruited. While this could be solely due to a lack of Mis18 $\beta$ presence at the array, it is clear that HJURP recruitment to chromatin absolutely requires the presence of Mis18 $\alpha$ and Mis18 $\beta$. 
Figure 2-5. Mis18 $\alpha$ and Mis18 $\beta$ recruit HJURP to centromeres for CENP-A deposition.

(A) Anti-Mis18BP1 western blot indicating the successful depletion of endogenous Mis18BP1 after siRNA treatment. An anti-tubulin antibody was used as a loading control. (B) Representative images of mCherry-LacI-Mis18 $\alpha$ targeted to the array and the recruitment of GFP-Mis18 $\beta$ and HA-HJURP in Mis18BP1 siRNA or GAPDH control siRNA treated cells. Enlarged images highlight recruitment of GFP-Mis $18 \beta$ and HAHJURP to the array. Scale bar $=5 \mu \mathrm{m}$. (C) Graph of the average percentage of transfected cells that recruited HA-HJURP to the array with respect to siRNA treatment. Error bars display the standard deviation between replicates. (D) Representative images of mCherry-LacI-Mis1 $8 \alpha$ constructs targeted to the array and the recruitment of GFPMis $18 \beta$ and HA-HJURP in the presence and absence of the C-terminal coiled-coil domain of Mis18 $\alpha$. Enlarged images highlight recruitment of GFP-Mis18 $\beta$ and HAHJURP to the array. Scale bar $=5 \mu \mathrm{m}$. (E) Graph of the percentage of transfected cells that recruited HA-HJURP to the array with respect to siRNA treatment. 

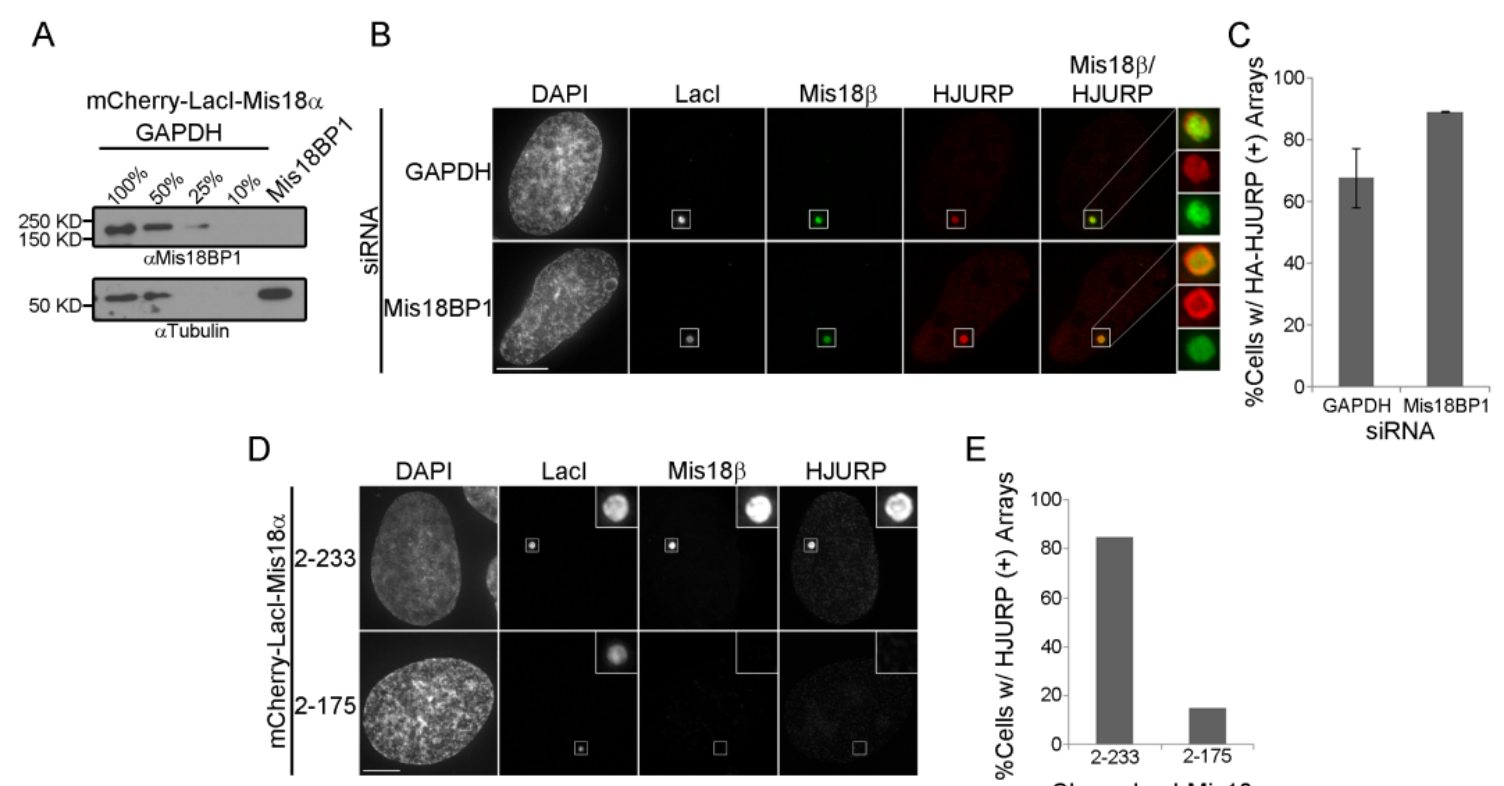

E

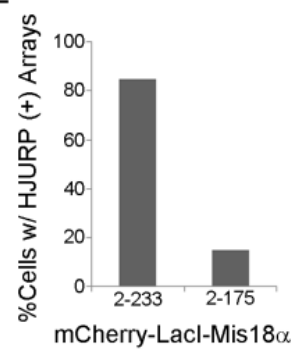

Figure 2-5 


\section{Discussion}

The Holliday junction recognition protein, HJURP, was previously found to interact specifically with prenucleosomal CENP-A/H4 [66, 83]. In human cells, HJURP was also found to be necessary and sufficient for the deposition of newly synthesized CENP-A nucleosomes in vivo and to assemble CENP-A nucleosomes onto plasmid DNA in vitro $[18,66,83]$. These results strongly indicated that HJURP is the assembly factor for CENP-A. Cell cycle analysis of HJURP localization showed that HJURP localizes to centromeres in G1, which follows the late anaphase recruitment of the human Mis18 complex $[66,83]$. Indeed, we determined that human Mis18 $\alpha$ and Mis18BP1 are required for the centromeric localization of HJURP as siRNA depletions of either protein abolished HJURP's ability to interact with G1 centromeres. Similar studies in frogs and fission yeast showed that the dependency of HJURP homologs on the Mis18 proteins is highly conserved $[100,156,157]$.

In addition, we have found that the G1 regulated deposition of CENP-A is controlled by Cdk1 and Cdk2 activity. When Cdk activity is high in late G1 and throughout G2/M, Mis18BP1 is unable to associate with centromeric chromatin. Once Cdk activity sharply declines during anaphase onset, there is a change in Mis18BP1 phosphorylation status, and the complex is able to associate with the centromere to recruit downstream CENP-A deposition factors. When Cdk1 and Cdk2 are artificially inhibited at other points of the cell cycle, the Mis18 complex and its downstream effectors aberrantly associate with the centromere and deposit newly synthesized CENP-A outside of G1. This indicates that not only is centromere location spatially regulated by the Mis 18 
complex, but also the phosphorylation of Mis $18 \mathrm{BP} 1$ by $\mathrm{Cdk} 1 / \mathrm{Cdk} 2$ temporally regulates CENP-A deposition. While the localization of the Mis18 complex is affected by the inhibition of $\mathrm{Cdks}$, it is conceivable that $\mathrm{Cdk}$ phosphorylation may regulate multiple players in this pathway.

Finally, our data suggest that the Mis18 complex may have multiple functions in the CENP-A deposition pathway. Mis18BP1 dynamically binds to G1 centromeres, while Mis $18 \alpha$ remains stably bound to centromeric chromatin. In addition, only Mis $18 \alpha$ and Mis18 $\beta$ are absolutely required for HJURP localization to centromeric chromatin. The role of Mis18BP1 appears to be required only for Mis18 $\alpha$ and Mis18 $\beta$ localization in human cells. In Figure 2-6, we propose a model by which the Mis 18 complex senses the biochemical changes as a cell exits mitosis, through the phosphorylation status of Mis18BP1. Once Cdk activity levels decrease, Mis18BP1 is dephosphorylated and the Mis 18 complex associates with the centromere. Mis18 $\alpha$ and Mis18 $\beta$ interact together via a C-terminal coiled-coil domain in each protein and recruit the CENP-A assembly factor, HJURP. Finally CENP-A is deposited into centromeric chromatin where it is subject to further maturation processes to ensure stable incorporation into centromeric chromatin.

Centromere organization varies greatly across evolution, and there are many correlations between centromere architecture and the proteins involved with CENP-A homolog deposition. Budding yeast, which have a point centromere, do not possess a Mis 18 homolog. Instead, the $S$. cerevisiae $\mathrm{Scm} 3$ protein binds AT-rich DNA at the centromere, which may serve as a possible recruitment mechanism [158]. In humans and fission yeast, which both harbor regional centromeres; the Mis18 homologs are required 
for the recruitment of HJURP/Scm 3 to centromeres [18, 91]. Even among organisms that have regional centromeres there are differences in the mechanisms by which the Mis18 proteins recruit HJURP/Scm3. A single Mis 18 protein is present in $S$. pombe while two paralogs are found in higher eukaryotes [90]. Moreover, the Mis18BP1 subunit of the complex appears to be specific to higher eukaryotes, as an $S$. pombe homolog has not been identified. While C. elegans, which have regional but holocentromeres, have only a Mis18BP1 homolog [92]. Therefore, the components of the CENP-A deposition pathway have evolved for each organism's specific centromere architecture. The presence of the separate Mis 18 proteins and their differential binding behavior may indicate different, yet essential roles in ensuring the maintenance of human centromeric chromatin from cell cycle to cell cycle. This concept will be further addressed by data presented in chapters 3 and 4. 
Figure 2-6. The deposition of newly synthesized CENP-A occurs during G1.

The schematic shows a model of CENP-A deposition into centromeric chromatin during the G1 phase of the cell cycle. Cdk activity decreases after the exit from mitosis which correlates with the recruitment of the CENP-A deposition factors, the Mis18 complex and HJURP. Finally, the association of Rsf1/Snf2 and MgcRacGap with the centromere enables the establishment of fully stable CENP-A nucleosomes through chromatin remodeling mechanisms and GTP-GDP cycling. 

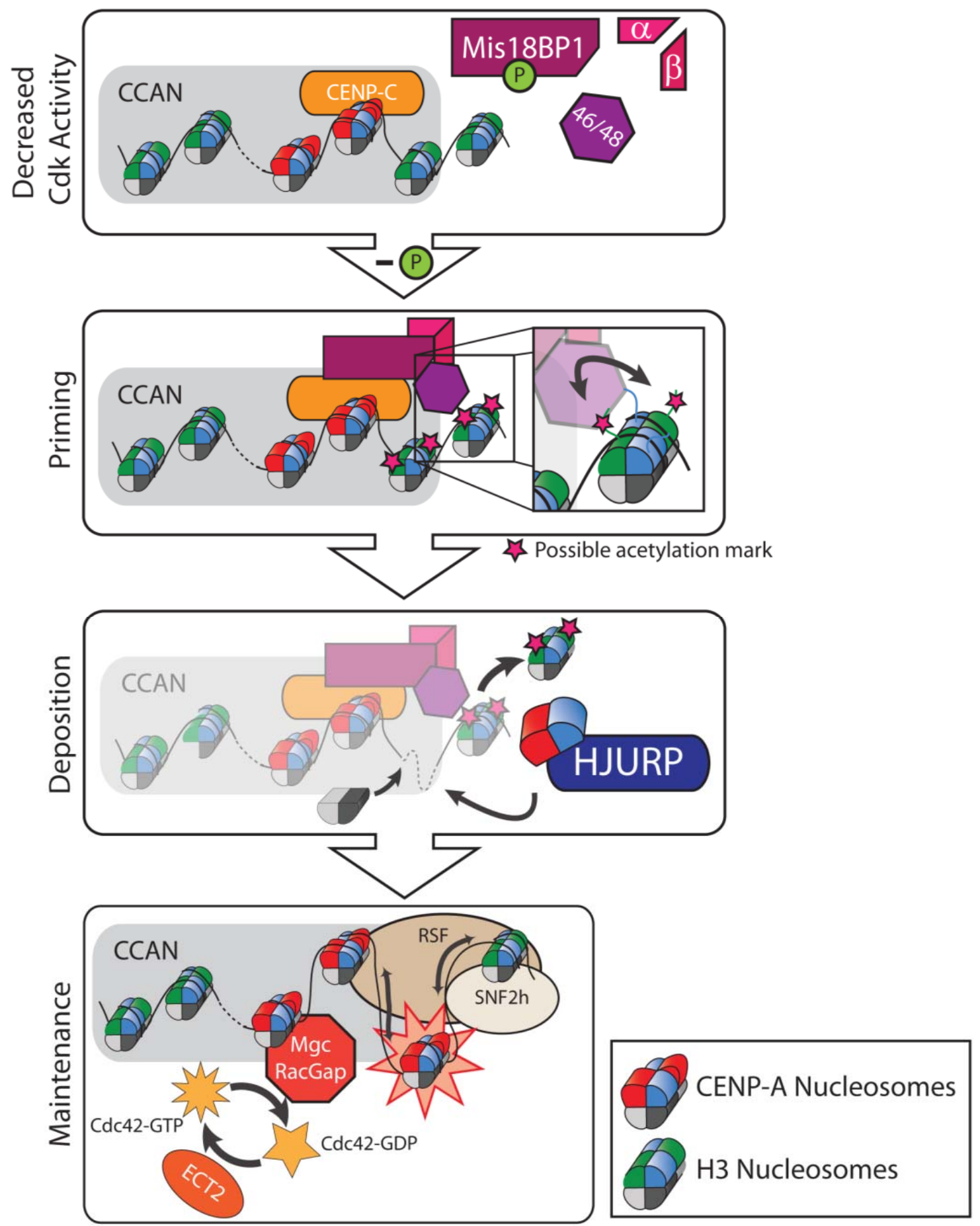

Figure 2-6

Published as Figure 4 in Stellfox, ME, et al. (2012) Cellular and molecular life sciences. 


\section{Materials and Methods}

\section{Tissue culture}

HeLa derived cells lines were cultured in DMEM High Glucose (Gibco) supplemented with 10\% heat inactivated FBS (Optima, Atlanta Biologicals) and $1 \mathrm{X}$ Penicillin/Streptomycin (Gibco). U2OS derived cell lines were cultured in DMEM High Glucose GlutaMAX ${ }^{\mathrm{TM}}$ (Gibco) supplemented with 10\% FBS and 1X Penicillin/Streptomycin. Cells were incubated at $37^{\circ} \mathrm{C}$ in $5 \% \mathrm{CO}_{2}$ and $85 \%$ humidity. A stable GFP-Mis18BP1 expressing cell line was generated using the Flp-In system (Life Technologies) in a previously established HeLa T-REX Flp-In cell lines (S. Taylor, University of Manchester). Recombined cell lines were maintained in media supplemented with $200 \mu \mathrm{g} / \mathrm{mL}$ Hygromycin B.

\section{General immunofluorescence and image acquisition methods}

Cell lines were grown on poly-lysine coated glass coverslips, fixed for $10 \mathrm{~min}$ with 4\% formaldehyde in PBS (137 mM NaCl, $2.7 \mathrm{mM} \mathrm{KCl}, 5.4 \mathrm{mM} \mathrm{Na}_{2} \mathrm{HPO}_{4}, 1.8 \mathrm{mM}$ $\mathrm{KH}_{2} \mathrm{PO}_{4}$ ), warmed to $37^{\circ} \mathrm{C}$ and then quenched for $5 \mathrm{~min}$ with $100 \mathrm{mM}$ Tris $\mathrm{pH}$ 7.5. Coverslips were blocked for $1 \mathrm{~h}$ in blocking buffer: $2 \%$ heat inactivated FBS, $2 \%$ BSA, and $0.1 \%$ Triton-X100 in PBS. Primary antibodies were incubated for $1 \mathrm{~h}$ in blocking buffer: anti-CENP-A mouse monoclonal antibody (ab13939, Abcam) at $1 \mu \mathrm{g} / \mathrm{mL}$ and anti-CENP-T rabbit polyclonal antibody serum (D. Cleveland, Ludwig Institute for Cancer Research) at 1:2000. Coverslips were washed three times for 5 min with $0.1 \%$ Triton-X100 in PBS. Secondary antibodies were diluted in blocking buffer and were 
incubated with the coverslips for $1 \mathrm{~h}$ (Alexa-647 conjugated goat-anti-mouse at 0.375 $\mu \mathrm{g} / \mathrm{mL}$, Cy5 conjugated goat-anti-rabbit conjugated at $0.375 \mu \mathrm{g} / \mathrm{mL}, \mathrm{Cy} 3$ conjugated goat-anti-mouse at $0.75 \mu \mathrm{g} / \mathrm{mL}$; Jackson ImmunoResearch Laboratories, Inc.). Cells were washed as above and then incubated in PBS containing $0.2 \mu \mathrm{g} / \mathrm{mL}$ of DAPI for $5 \mathrm{~min}$. Coverslips were rinsed with PBS prior to mounting onto glass slides with Prolong Gold Antifade Reagent (Life Technologies).

Fluorescence microscopy images were acquired using a DeltaVision deconvolution microscope (GE Healthcare Lifesciences) equipped with a CoolSNAP $\mathrm{HQ}^{2}$ camera (Photometrics). Images were collected using either a 60X (numerical aperture $=1.42$; Olympus) or a $100 \mathrm{X}$ (numerical aperture $=1.40$; Olympus) oil immersion lens. All images within an experiment were acquired using identical exposure times across all conditions. Images shown in figure panels are deconvolved maximum projections and scaled equally.

\section{siRNA treatment and sample preparation}

For western blot analysis of protein levels, HeLa cell lines were plated at $8 \times 10^{5}$ cells in six-well plates. The next day, cells were transfected with $5 \mathrm{nM}$ siRNA in OptiMEM media (Gibco) and RNAiMAX (Invitrogen). Mock transfections contained RNAiMAX transfection reagent without siRNA. The following day, 1/3 of the plating volume of DMEM with 10\% heat inactivated FBS was added. Forty-eight hours after siRNA treatment, cells were harvested with 3 mM EDTA, counted and whole cell lysates were made in SDS-PAGE sample buffer (60 mM Tris HCl/SDS pH 6.8, 5\% glycerol, 
$1.67 \%$ SDS, $0.01 \%$ bromophenol blue, $1 \% \beta$-mercaptoethanol). Lysates from $1 \times 10^{5}$ cells were run out on $10 \%$ SDS-PAGE gel and transferred to nitrocellulose. Blots were incubated in primary antibody overnight at $4^{\circ} \mathrm{C}$ and in secondary for $1 \mathrm{~h}$ also at $4^{\circ} \mathrm{C}$ (Figure 2-1) or at room temperature for the remaining figures.

\section{Fluorescent microscopy analysis of Mis18 and HJURP depletions}

HeLa GFP-HJURP cells were scored as single-plane images. Nuclei with all CENP-T foci colocalized with GFP-HJURP were considered "GFP-HJURP positive”. GFP-Mis 18 $\alpha$ loading was analyzed using MetaMorph 7.7. Using maximum projection images from deconvolved z-stacks, nuclei were visually divided into three categories: fully loaded (all CENP-T and GFP-Mis $18 \alpha$ foci colocalized), partially loaded (at least one, but $<80 \%$, of CENP-T foci colocalized with GFP-Mis $18 \alpha$ ) and unloaded (no CENPT and GFP-Mis $18 \alpha$ colocalization). The maximum GFP intensities were plotted for every nucleus in each category. The maximum intensity of partially loaded and unloaded nuclei was always below 7600 A.U.s. Thus, only cells that had GFP-Mis $18 \alpha$ at all centromeres with a maximum intensity above 7600 A.U.s were considered to be GFPMis $18 \alpha$ positive.

\section{Nocodazole treatments for Mis18 $\beta$}

HeLa cells growing in DMEM 1X high glucose media (Gibco) were treated with $100 \mathrm{ng} / \mathrm{mL}$ nocodazole or vehicle control (DMSO) for $12 \mathrm{~h}$. Mitotic cells were isolated by shake-off from nocodazole treated cells, and vehicle control cells were collected with 
$3 \mathrm{mM}$ EDTA-PBS. Cell pellets were resuspended in 1X sample buffer $(60 \mathrm{mM}$ Tris $\mathrm{HCl} / \mathrm{SDS}$ pH 6.8, 5\% glycerol, 1.67\% SDS, 0.01\% bromophenol blue, 1\% $\beta$ mercaptoethanol) at a concentration of $1 \times 10^{7}$ cells $/ \mathrm{mL}$ and disrupted using an insulin syringe. $1 \times 10^{5}$ cells per lane were run on either an $8 \%($ Mis $18 \mathrm{BP} 1)$ or a $12 \%(\mathrm{H} 3 \mathrm{~S} 10 \mathrm{p})$ SDS-PAGE gels and blotted to nitrocellulose using standard approaches. The nitrocellulose was blocked for $1 \mathrm{~h}$ in blocking buffer: 5\% milk in TBST (137 mM NaCl, 2.7 mM KCl, $25 \mathrm{mM}$ Tris Base, 0.1\% Tween 20). Primary antibodies were incubated overnight in blocking buffer at $4^{\circ} \mathrm{C}[0.2 \mu \mathrm{g} / \mathrm{mL}$ Mis18BP1 (Bethyl Laboratories, Inc., Cat\# A302-825A or Cat\# A302-824A) and $0.2 \mu \mathrm{g} / \mathrm{mL}$ histone H3 phosphorylated serine 10 (H3S10p clone 3H10) (Millipore, 05-806)]. HRP conjugated secondary antibodies were incubated in blocking buffer at $40 \mathrm{ng} / \mathrm{mL}$ for $1 \mathrm{~h}$ at room temperature (Goat-antimouse, Cat\# 115-035-003; Goat-anti-rabbit, Cat\# 111-0.35-003, Jackson ImmunoResearch Laboratories, Inc.). HRP was detected using the West Pico ECL Regent (Pierce) chemiluminescent substrate.

\section{Mis18BP1 phospho-mutant transfection and western blot}

HeLa cells were plated at a density of $1 \times 10^{5}$ cells per well in a 6-well plate. Cells were transfected the following day with $400 \mathrm{ng}$ of plasmid DNA using Effectene transfection reagent (Qiagen) in Opti-MEM reduced serum media (Gibco) according to the manufacturer's directions. $29 \mathrm{~h}$ later, cells were blocked for approximately $12 \mathrm{~h}$ in either $100 \mathrm{ng} / \mathrm{mL}$ nocodazole or DMSO as a vehicle control. Cells were harvested and lysed in SDS sample buffer and $1 \times 10^{5}$ cells per lane were run on a $6 \%$ SDS-PAGE gel. 
Anti-GFP antibody for immunblotting was used at a 1:1000 dilution overnight at $4{ }^{\circ} \mathrm{C}$. Secondary antibody was at a 1:10000 dilution of goat-anti-rabbit-HRP (Jackson ImmunoResearch) for $1 \mathrm{~h}$ at room temperature. Protein levels were analyzed with West Pico ECL Reagent (Pierce).

\section{CIP treatment and western blotting}

For CIP treatments, lysates were prepared from a HeLa cell line stably expressing LAP-Mis18BP1 that had been blocked approximately $12 \mathrm{~h}$ with $100 \mathrm{ng} / \mathrm{mL}$ nocodazole or DMSO as a vehicle control. Loosely adhered mitotic cells and were combined with adhered cells harvested with $3 \mathrm{mM}$ EDTA-PBS for $10 \mathrm{~min}$ at room temperature. Cells were resuspended in buffer containing 75 mM HEPES pH 7.5, $1.5 \mathrm{mM}$ EGTA, $1.5 \mathrm{mM}$ $\mathrm{MgCl}_{2}, 150 \mathrm{mM} \mathrm{KCl}, 15 \%$ glycerol, 0.075\% IGEPAL, $10 \mathrm{mM}$ imidazole, $200 \mu \mathrm{M}$ sodium orthovanadate, $5 \mathrm{mM}$ sodium fluoride, $50 \mathrm{mM} \beta$-glycerophosphate and Roche Complete EDTA-free Protease Cocktail. Cell suspensions were then sonicated on ice in $30 \mathrm{~s}$ cycles for a total of $2 \mathrm{~min}$. Lysates were centrifuged for $10 \mathrm{~min}$ at $1000 \mathrm{~g}$ at $4^{\circ} \mathrm{C}$ and the supernatants were passed $5 \mathrm{X}$ over a column containing His-tagged GFP binding protein (GBP) bound to Ni-NTA agarose. Proteins bound to the GBP beads were washed, resuspended in $30 \mu \mathrm{L}$ wash buffer (50 mM HEPES pH 7.5, $1 \mathrm{mM} \mathrm{MgCl2,} 300 \mathrm{mM} \mathrm{KCl,}$ $10 \%$ glycerol) and incubated with $10 \mathrm{mM}$ sodium orthovanadate (MP Biomedicals) and/or 60 units of calf intestinal alkaline phosphatase (CIP, New England Biolabs) for $1 \mathrm{~h}$ at $37^{\circ} \mathrm{C}$. Reactions were stopped by the addition of SDS sample buffer. Western blots represent 11\% of total input. Anti-Mis18BP1 antibody (Bethyl Labs) was a 1:5000 
dilution overnight at $4^{\circ} \mathrm{C}$. Secondary antibody was a 1:10000 dilution of goat-anti-rabbitHRP (Jackson ImmunoResearch) for $1 \mathrm{~h}$ at room temperature. Protein levels were analyzed with West Pick ECL Reagent (Pierce).

\section{GFP-Mis18BP1 localization and CENP-A-SNAP tag experiments}

As these experiments were done in collaboration with the Jansen laboratory, please refer to the materials and methods section of:

Silva, MC, Bodor, DL, Stellfox, ME, Martins, NM, Hochegger, H, Foltz, DR and Jansen, LE. Cdk activity couples epigenetic centromere inheritance to cell cycle progression. Developmental cell. (2012) 22:52-63.

\section{Fluorescence recovery after photobleaching (FRAP)}

HeLa cells stably expressing GFP-Mis18 $\alpha$ or transiently transfected with GFPMis18BP1 were grown on glass-bottomed culture dishes (MatTek Corporation) in normal growth media. Prior to imaging, growth media was replaced with Leibovitz's L-15 medium without phenol red (Gibco) supplemented with 10\% heat inactivated FBS (Optima, Atlanta Biologicals). Photobleaching was conducted using a Zeiss LSM 510 UV Confocal Microscope with a 100X oil immersion lens (Plan-Apochromat). Cells with GFP-Mis18 $\alpha$ or GFP-Mis18BP1 localized to centromeres were selected for analysis. At least two pre-bleach images were collected to assess the initial fluorescence intensity. Individual centromeres were bleached with 70 iterations of the $488 \mathrm{~nm}$ laser and the fluorescence recovery at the centromere was assessed over time. Images were collected at $10 \mathrm{~s}$ intervals throughout the experiment. Fluorescence recovery at photobleached 
centromeres was analyzed using ImageJ. Fluorescence data were normalized to account for sample bleaching based on previous methods [159]. Briefly, fluorescence intensity was measured within the entire nucleus $(\mathrm{T})$, within the photobleached region $(\mathrm{PB})$ and within a background region (BG) outside the nucleus. BG intensity was subtracted from the T and PB intensities at each time point. Pre-bleach intensity measurements of the entire cell and of the photobleached region were averaged to yield $\mathrm{T}_{\text {pre }}$ and $\mathrm{PB}_{\text {pre }}$. Normalized values at each time point $(\mathrm{t})$ were calculated according to $\left(\mathrm{T}_{\text {pre }} / \mathrm{T}_{\mathrm{t}}\right) *\left(\mathrm{~PB}_{\mathrm{t}} / \mathrm{PB}_{\mathrm{pre}}\right)$. The fluorescence recovery intensity was expressed as recovery relative to the $\mathrm{PB}_{\text {pre }}$ (equal to 1 ) and the intensity immediately after photobleaching (equal to 0 ). Fluorescence recovery was averaged across 18 centromeres for GFP-Mis18 $\alpha$ and 10 centromeres for GFP-Mis18BP1. Average fluorescence recovery data was fit to a single exponent curve $A^{*}\left(1-\mathrm{e}^{-\mathrm{kt}}\right)$ using 'nls' function in $\mathrm{R}$ [159].

\section{Plasmid transfections}

U2OS cells were plated at a density of either 10,400 cells $/ \mathrm{cm}^{2}$ or 35,000 cells $/ \mathrm{cm}^{2}$ in a 6-well plate or 24-well plate respectively and allowed to attach to the plate and coverslips for DNA transfections. The following day, cells were transfected with either 1 $\mu \mathrm{g}$ plasmid DNA (6-well format) or with $300 \mathrm{ng}$ (24-well format) using Lipofectamine 2000 (Life Technologies) in Opti-MEM (Gibco) according the manufacturer's protocol. For centromere localization counts of the GFP-Mis18 $\alpha$ and GFP-Mis $18 \beta$ constructs, cells were transfected with a 10:1 ratio of GFP construct to RFP-H2B plasmid, which served as a transfection marker. For the LacO array experiments, mCherry-LacI constructs were 
always transfected either in a 1:2 ratio with GFP proteins, or in a 1:2:2 ratio with the GFP-Mis $18 \beta$ and HA-HJURP constructs. The transfection media was removed after $10-$ $12 \mathrm{~h}$ and replaced with fresh growth media. Cells were harvested with $3 \mathrm{mM}$ EDTA-PBS for western blot analysis or fixed for immunofluorescence $48 \mathrm{~h}$ post-transfection. 


\section{Chapter 3 - Distinct Roles of Mis18 $\alpha$ and Mis18 $\beta$ in Cell Cycle Regulation of Centromere Specification}

This chapter is based on the manuscript currently under review:

Stellfox, M.E., I.K. Nardi, C.M. Knippler, D.R. Foltz. "Distinct roles of Mis $18 \alpha$ and Mis18 $\beta$ in cell cycle regulation of centromere specification". 


\begin{abstract}
The deposition of newly synthesized CENP-A nucleosomes into centromeric chromatin occurs during G1, and it is controlled by the CENP-A specific assembly factor, Holliday junction recognition protein (HJURP), and the Mis18 complex, which consists of Mis18 $\alpha$, Mis18 $\beta$ and Mis18 binding protein 1 (Mis18BP1/hsKNL2). We show that Mis $18 \alpha$ and Mis18 $\beta$ interact as a complex that is separate from Mis18BP1 in mitosis, prior to the recruitment of the complex to the centromere. Although Mis18 $\alpha$ and Mis18 $\beta$ are considered homologous proteins, we find that they mediate distinct interactions that link the Mis 18 complex to centromeric chromatin during G1. Mis18 $\alpha$ interacts directly with the amino terminus of Mis18BP1. Mis18 8 mediates a physical interaction with CENP-C in a cell cycle dependent manner. Both of these interactions require the Yippee/Mis18 domain of Mis18 $\alpha$ and Mis18 $\beta$. The N-terminus of Mis18BP1, containing both the Mis18 $\alpha$ and CENP-C binding domains, is necessary and sufficient for centromeric localization and for CENP-A deposition at centromeres. Therefore, the interaction of Mis18BP1 with Mis18 $\alpha$ and the cell cycle regulated interaction between Mis $18 \beta$ and CENP-C generate robust centromeric localization that leads to CENP-A deposition.
\end{abstract}




\section{Introduction}

The centromere is the chromosomal region that dictates kinetochore formation during mitosis. Centromere location is specified epigenetically in most eukaryotes, and the histone $\mathrm{H} 3$ variant, centromere protein $\mathrm{A}(\mathrm{CENP}-\mathrm{A})$ is considered to be the epigenetic marker of centromeric chromatin $[160,161]$. CENP-A deposition in human cells is separated from the S phase deposition of canonical nucleosomes. Parental CENP-A is retained during DNA replication while newly synthesized CENP-A is deposited during the following G1 $[78,84,85,93,162]$. Therefore, cells proceed through G2 and mitosis with the centromere of each daughter chromatid containing half of the full complement of CENP-A. Only after mitotic exit are newly synthesized CENP-A nucleosomes deposited into centromeric chromatin.

Mis18 is a highly conserved family of proteins present from yeast to humans. Mis18 association with the centromere is the earliest known step in CENP-A deposition $[90,91]$. Human cells contain two homologs of the fission yeast Mis18 protein, Mis18 $\alpha$ and Mis18 $\beta$ [90]. Both Mis18 $\alpha$ and Mis $18 \beta$ contain a highly conserved Yippee/Mis18 (YM) (PFAM: PF03226) domain that is characterized by a set of cysteine residues, whose replacement with alanine, in Mis18 $\alpha$, disrupts its centromeric recruitment and function $[90,163]$. In humans and many higher eukaryotes, Mis18 $\alpha$ and Mis18 interact with another protein, Mis18 binding protein 1 (Mis18BP1 also known as KNL-2 and M18BP1), which is required for Mis18 $\alpha$ and Mis18 $\beta$ localization as well as downstream CENP-A deposition [90, 92]. Mis18BP1 contains a highly conserved SANT (Swi3, Ada2, N-Cor, and TFIIIB) domain as well as a SANT-associated (SANTA) domain [92, 
111]. The Mis18 proteins are mutually dependent on each other for localization, and over time, the loss of Mis18BP1 induces the destabilization of Mis18 $\alpha$ and Mis18 [90]. In addition, all three proteins are required for the deposition of newly synthesized CENP-A nucleosomes by recruiting the CENP-A specific chromatin assembly factor, HJURP [18, $66,83,90,100,151]$. However, it is unknown how the three proteins of the Mis 18 complex physically interact with each other, and whether the interactions are cell cycle regulated.

The cell cycle timing of CENP-A deposition is mediated through the recruitment of the Mis18 complex to the centromere. Centromeric localization of Mis18BP1 is inhibited by $\mathrm{Cdk} 1 / \mathrm{Cdk} 2$ activity, which declines rapidly after anaphase onset thereby allowing Mis 18BP1 to localize to centromeres and initiate CENP-A deposition in early G1 [164]. However, many questions remain regarding the mechanisms that mediate the interaction of the Mis18 complex with centromeric chromatin. In frogs and mice, Mis18BP1 physically interacts with the constitutive centromere protein, CENP-C [99, 100]. This interaction is currently the only known physical interaction that contributes to the specific centromeric localization of the Mis18 complex; however, the sufficiency of the Mis18BP1-CENP-C interaction to support the highly regulated recruitment of the Mis18 complex in human cells remains unclear.

In this study, we show that the Mis18 $\alpha$ and Mis18 $\beta$ paralogs have distinct binding partners, which serve to link the Mis18 complex to centromeric chromatin through several physical interactions. We find that during mitosis, Mis18 $\alpha$ and Mis18 $\beta$ form a separate complex that does not include Mis18BP1. After mitotic exit, Mis18 $\alpha$ interacts 
directly with the amino terminus of Mis18BP1 while Mis18 $\beta$ physically interacts with CENP-C in a cell cycle dependent manner. Fragments of Mis18BP1 that only include the previously identified CENP-C binding domain are not sufficient to localize Mis18BP1 to human centromeres. In addition, neither the conserved SANT nor SANTA domains are required for centromere localization. Full localization of the Mis18 complex requires the previously identified CENP-C binding domain as well as the Mis18 $\alpha$ interacting domain of Mis18BP1. This joint interaction between the Mis 18 complex proteins and CENP-C mediates the tightly regulated localization of the Mis18 complex and subsequent CENPA deposition. 


\section{Results}

\section{The N-terminus of Mis18BP1 is sufficient for centromeric localization}

\section{and CENP-A recruitment}

In order to determine the domains of Mis18BP1 that were required for its localization to centromeric chromatin, I expressed a series of GFP-tagged fragments of human Mis18BP1 in randomly cycling U2OS cells (Figure 3-1A and Supplemental Figure S3-1A). Recruitment to endogenous centromeres was determined by colocalization with either CENP-T, or CENP-A (Figure 3-1B). Full-length Mis18BP1 was found at centromeres in $21.0 \pm 12.9 \%$ of randomly cycling cells, which is consistent with its limited presence at centromeres from late telophase through mid-G1 phase (Figure 31C) $[90,133]$. Only the fragment of Mis18BP1, which contained the N-terminus and the CENP-C binding domain (Mis18BP1-721) displayed centromere recruitment similar to that of the full-length protein $[99,100]$. Full-length Mis18BP1 displayed a greater variation in its centromeric recruitment than GFP-Mis $18 \mathrm{BP} 1^{2-721}$ between experiments, but the difference between the two GFP constructs was not statistically significant $(\mathrm{p}=$ 0.32). A fragment of Mis18BP1 containing the entire CENP-C binding domain (Mis18BP1 ${ }^{476-721}$ ), but not the N-terminus, was not recruited to centromeres. This suggests that although this region of Mis18BP1 is able to interact with CENP-C, this interaction is not sufficient to localize the Mis 18 complex to the human centromere [ 99 , 100]. In addition, the inclusion of the conserved SANTA domain (Mis $18 \mathrm{BP}^{183-721}$ ) with the CENP-C binding region was not sufficient to recruit Mis18BP1 to centromeres. In fact, deleting the conserved SANTA domain alone did not abolish the centromeric 
localization of the otherwise full-length protein $\left(\operatorname{Mis} 18 \mathrm{BP} 1^{\Delta 377-475}\right)$. This agrees with recent data in Arabidopsis showing that the SANTA domain is not required for centromeric localization of Mis18BP1 [165]. Interestingly, the C-terminal Mis18BP1 fragment (Mis18BP1 $1^{717-1132}$ ), which contains the well-conserved SANT domain, was also not required for centromere recruitment.

The human Mis 18 complex localizes to centromeres after mitotic exit and remains at centromeres throughout G1, in a Cdk-regulated manner [90, 164]. Throughout the localization analysis of the GFP-Mis18BP1 fragments, I did not observe any centromeric recruitment that would suggest a change in the cell cycle regulation of the GFPMis18BP1 $1^{2-721}$ fragment (Figure 3-1). Cells with GFP positive centromeres always had the morphology of a G1 or interphase cell. Therefore, the first 721 amino acids of human Mis18BP1 are sufficient to interact with centromeric chromatin with normal cell cycle regulation.

Using siRNAs that target an exon in the C-terminal portion of Mis18BP1, I were able to selectively deplete endogenous Mis18BP1 to less than 5\% relative to controls, while maintaining exogenous expression of GFP-Mis18BP1 ${ }^{2-721}$ (Supplemental Figure S3-1, B and C). This allowed me to examine whether Mis18BP1 ${ }^{2-721}$ required endogenous Mis18BP1 to localize to centromeric chromatin. GFP-Mis18BP1 $1^{2-721}$ localized to centromeres in cells that were depleted of endogenous Mis18BP1 as efficiently as in cells that were treated with siRNA targeting GAPDH as a control (Supplemental Figure S3-1, $\mathrm{C}$ and D). Therefore, Mis18BP1 requires two domains for centromere targeting, the CENP-C binding domain (a.a. 476-721) and the N-terminal sequence, upstream of the 
SANTA domain (a.a. 2-376). These sequences are sufficient to fully recruit Mis18BP1 to centromeres $[99,100]$.

Mis18BP1 is required for the localization of HJURP and the subsequent deposition of newly synthesized CENP-A into centromeric chromatin [18, 90, 92]. While the first 721 amino acids of Mis18BP1 were sufficient for centromeric recruitment, the question remained as to whether this domain was sufficient to support new CENP-A deposition into centromeric chromatin. Therefore, I assayed whether GFP-tagged Mis18BP1 $1^{2-721}$ was sufficient to rescue CENP-A loss when endogenous Mis18BP1 was depleted by siRNA targeted to an exon of Mis18BP1 not present in Mis18BP1 $1^{2-721}$. Cells treated with GAPDH or Mis18BP1 siRNA were simultaneously rescued with either myristolated GFP (myr-GFP), as a negative control, or GFP-Mis18BP1 $1^{2-721}$ and stained for endogenous CENP-A. Mis18BP1 siRNA treated cells rescued with myr-GFP demonstrated a 50\% decrease in centromeric CENP-A (Figure 3-1, E and F). However, Mis18BP1 siRNA treated cells rescued with GFP-Mis18BP1 ${ }^{2-721}$ showed CENP-A levels similar to cells treated with control siRNA. This demonstrates that Mis18BP1 $1^{2-721}$, in addition to being required for centromeric recruitment, was also sufficient to direct CENP-A deposition to centromeres. 
Figure 3-1. Mis18BP1 N-terminus is required and sufficient for localization and function.

(A) Schematic of the GFP-tagged Mis18BP1 constructs used throughout Figure 3-1. (B) Representative images of GFP-tagged Mis18BP1 constructs expressed in randomly cycling U2OS cells. Centromeres (CEN) were identified using either anti-CENP-A (Mis18BP1 $1^{\Delta 377-475}$ ) or anti-CENP-T antibodies (all other constructs). Insets highlight GFP localization to an individual centromere. Scale bar $=5 \mu \mathrm{m}$. (C) Graph indicates the percentage of randomly cycling, transfected U2OS cells that have GFP signal localized to the centromere marker. Error bars show the standard deviation between replicates. Asterisks indicate a p-value $<0.05$ as compared to the centromeric localization of fulllength GFP-Mis18BP1 using the Student's t-test. (D) Representative images showing CENP-A recruitment to endogenous centromeres in U2OS cells treated with siRNA against GAPDH or Mis18BP1 and rescued by transfections with GFP-Mis18BP $1^{2-721}$ or myristolated GFP (myr-GFP). (E) Graph depicts the relative CENP-A intensity of cells treated with siRNA against endogenous Mis18BP1 compared to control cells treated with GAPDH siRNA. 
A

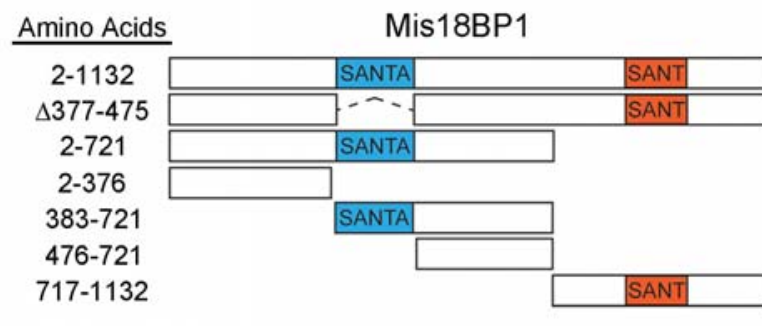

B GFP-Mis18BP1 DAPI CENP-T Mis18BP1 Merge

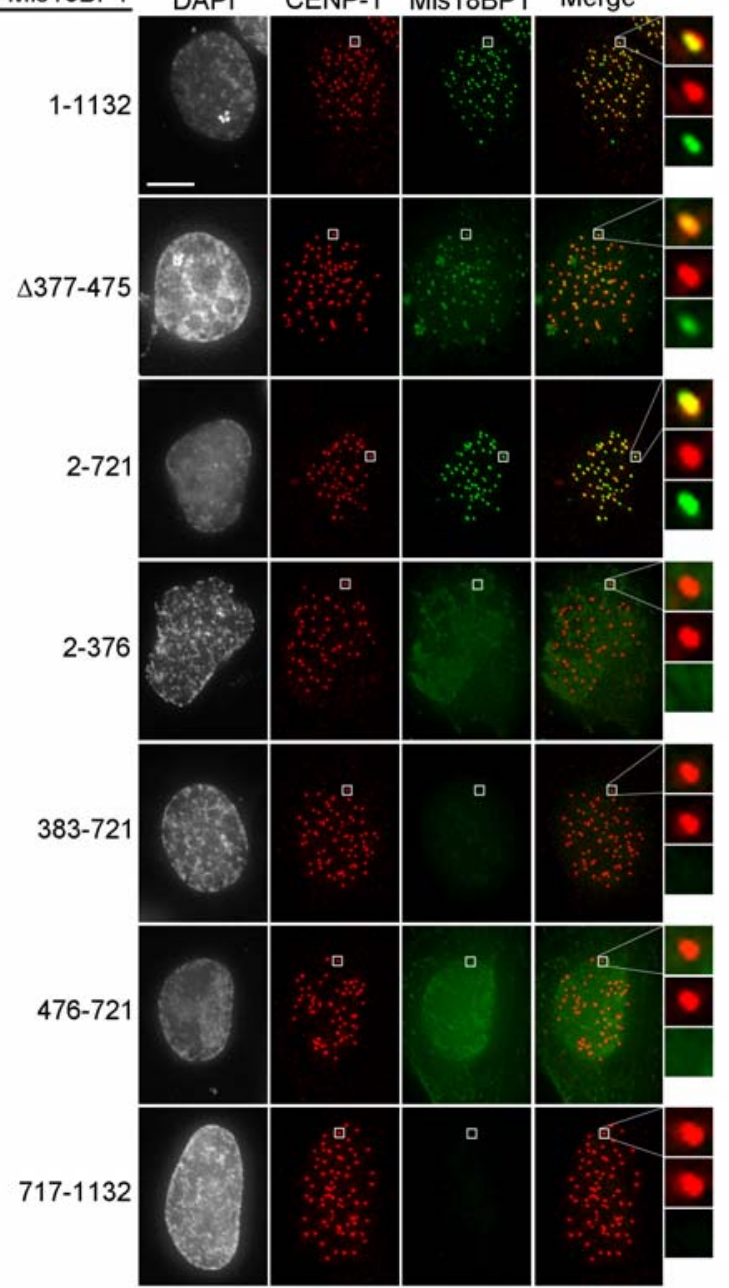

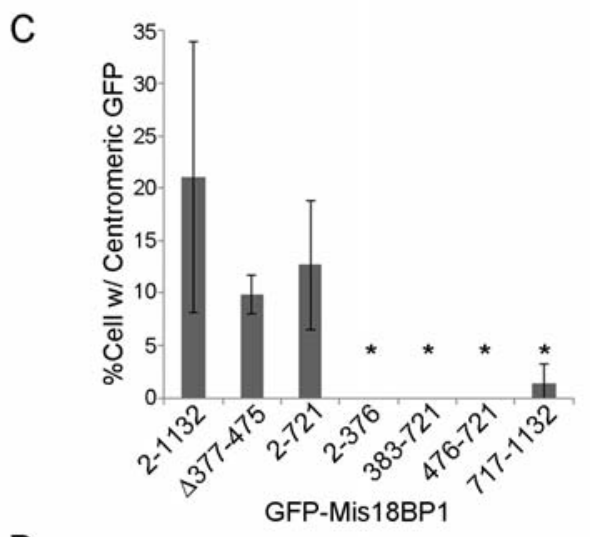

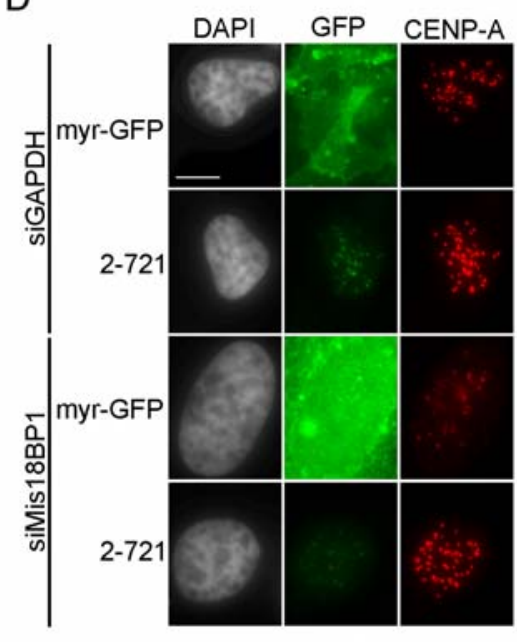

E

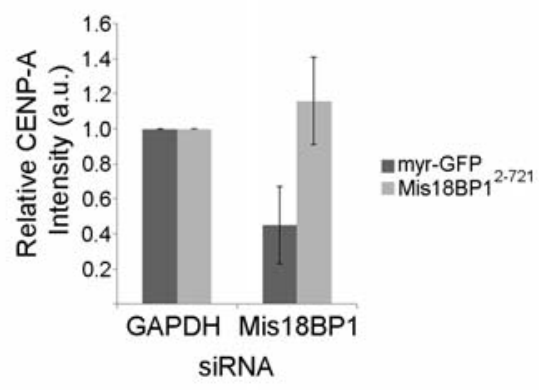

Figure 3-1 


\section{The extreme N-terminus of Mis18BP1 interacts with Mis18a}

The region of Mis18BP1 that supports the interaction with Mis18 $\alpha$ and Mis18 $\beta$ was previously unknown. All three proteins of the Mis 18 complex are required for CENP-A deposition [90]. Therefore, I hypothesized that a region of the N-terminal domain of Mis18BP1, which is sufficient for centromere localization and CENP-A retention, would also contain the interaction domain for Mis $18 \alpha$ or Mis18 $\beta$. Therefore, I assayed the interaction between Mis18BP1 and Mis18 $\alpha$ by co-immunoprecipitation. Nterminal and C-terminal Mis18BP1 fragments fused to an N-terminal 6xMyc-tag were co-expressed with GFP-Mis18 $\alpha$ in HEK cells. Proteins associated with Mis18BP1 were isolated from cell lysates using anti-Myc antibodies (Figure 3-2, A and B). Both fulllength Mis18BP1 and Mis18BP1 $1^{2-721}$ efficiently co-immunoprecipitated GFP-Mis18 $\alpha$; however, the C-terminal Mis18BP1 fragment failed to interact with Mis18 $\alpha$. This indicated that Mis18BP1 interacts with Mis18 $\alpha$ through its first 721 amino acids.

I used a complementary LacO interaction assay to validate the interaction between Mis18 $\alpha$ and Mis18BP1 and refine the interaction domain within Mis18BP1. Bait proteins were tagged with mCherry-LacI and targeted to a Lac operator (LacO) array integrated near the telomere of chromosome 1 [166] (Figure 3-2C). GFP-tagged prey proteins were co-expressed, and the interactions between the bait and prey proteins were assayed in an in vivo chromatin setting $[166,167]$. I assayed the recruitment of GFPMis18BP1 fragments to the LacO array when mCherry-LacI-Mis18 $\alpha$ was expressed as a bait protein (Figure 3-2, D and E). GFP-Mis18BP1 $1^{2-721}$ was robustly recruited to arrays targeted with mCherry-LacI-Mis18a, which was consistent with the co- 
immunoprecipitation assay. GFP-Mis $18 \mathrm{BP} 1^{2-376}$ was also recruited to the mCherry-LacIMis $18 \alpha$ arrays similarly to Mis $18 \mathrm{BP} 1^{2-721}$. Whereas, the GFP-Mis $18 \mathrm{BP} 1^{383-721}$ construct was not recruited to the arrays by mCherry-LacI-Mis18 $\alpha$. Therefore, the first 376 amino acids of Mis18BP1 mediate its physical interaction with Mis 18 $\alpha$, an interaction which contributes to the robust localization of the complex. 
Figure 3-2. Mis18BP1 interacts with Mis18 $\alpha$ through its extreme N-terminus.

(A) Schematic of the Mis18BP1 fragments used throughout Figure 3-2. (B) Immunoblot of anti-Myc co-immunoprecipitations from RIPA extracts of HEK cells co-expressing GFP-Mis $18 \alpha$ and 6xMyc-tagged Mis18BP1 constructs indicated by the amino acid numbers above each lane. (C) Diagram of the U2OS cell line containing a LacO/TetR array incorporated into chromosome 1. (D) Table of mCherry-LacI-tagged bait constructs and the GFP-tagged prey constructs analyzed in E and F. (E) Representative images of targeting mCherry-LacI alone or mCherry-LacI-Mis18 $\alpha$ to the LacO array and the recruitment of the GFP-tagged Mis18BP1 constructs. Anti-CENP-A antibody was used to mark endogenous centromeres. Scale bar $=5 \mu \mathrm{m}(\mathrm{F})$ Bar graph depicts the recruitment of the GFP-Mis18BP1 constructs to the array over the average nuclear background GFP signal, when either mCherry-LacI or mCherry-LacI-Mis $18 \alpha$ was used as bait. Error bars describe the standard deviation across replicates. Double asterisks indicate a p-value of $<$ 0.01 with respect to the recruitment of the GFP-Mis18BP1 fragments by mCherry-LacI alone, as calculated by the Student's t-test. 


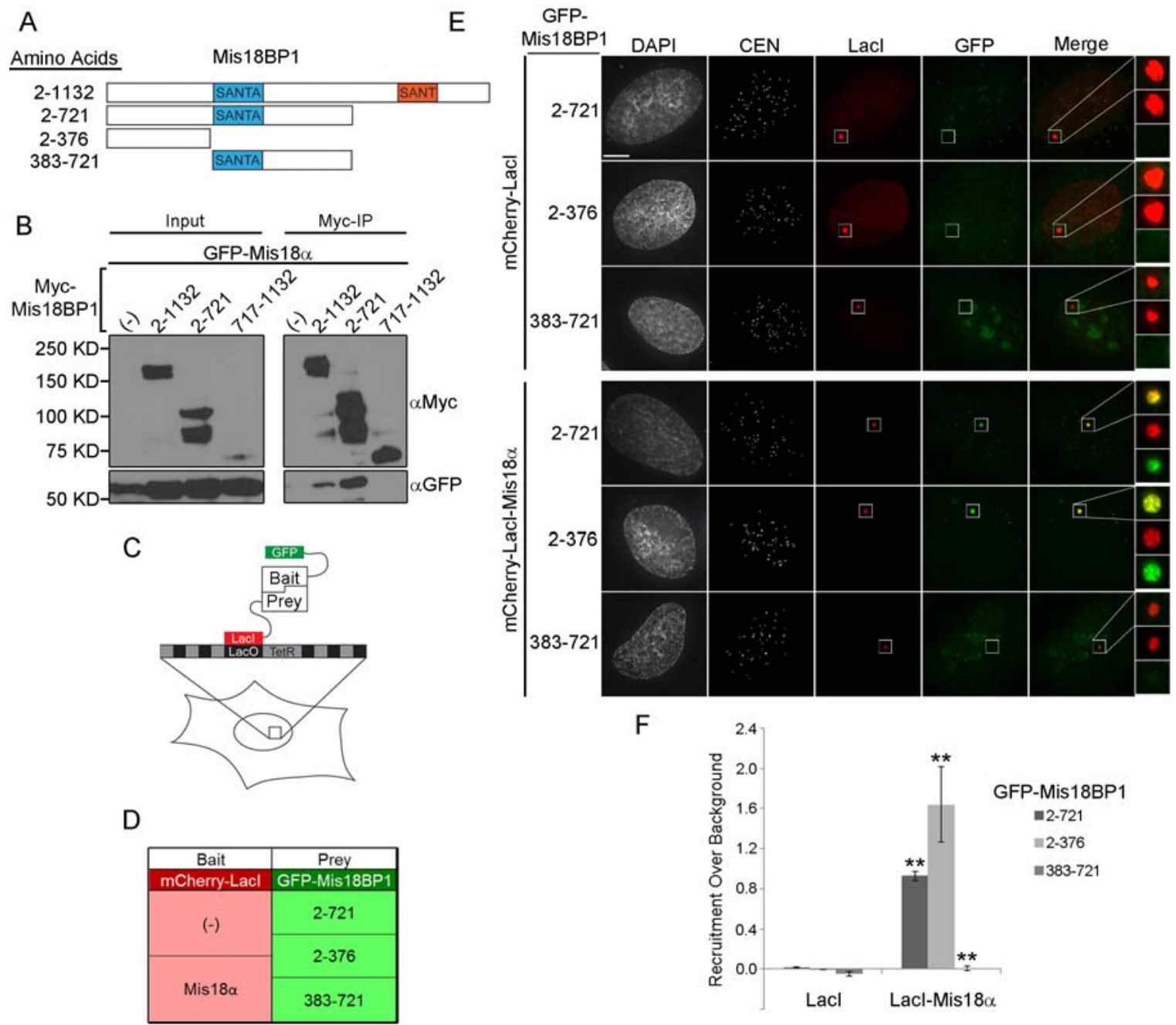

Figure 3-2 


\section{Mis18BP1 exists in a separate complex from Mis18 $\alpha$ and Mis18 $\beta$ prior}

\section{to $\mathbf{G 1}$}

Previous work demonstrated that Mis18BP1, Mis18 $\alpha$ and Mis18 $\beta$ are interdependent for their recruitment to centromeres [90]. I confirmed that Mis18BP1 requires both Mis $18 \alpha$ and Mis18 $\beta$ to be recruited to centromeres using siRNA depletion in a HeLa Flp-In T-Rex cell line that stably expressed GFP-Mis18BP1 (Figure 3-3A). Depleting Mis18 $\alpha$ or Mis18 $\beta$ reduced the percentage of cells with GFP-Mis18BP1 at centromeres compared to control siRNA (Figure 3-3, B and C). In addition, depletion of all three Mis 18 complex proteins resulted in a marked decrease in endogenous CENP-A deposition, indicating that each siRNA reduced the protein level and downstream function of its intended target (Supplemental Figure S3-2, A and B). Mis $18 \beta$ protein levels were clearly reduced in response to Mis18 $\alpha$ siRNA depletion, consistent with previous reports that indicated that Mis $18 \beta$ requires Mis $18 \alpha$ for protein stability (Figure 3-3B) [90]. Despite the absence of GFP-Mis18BP1 from centromeres, GFP-Mis18BP1 (upper band) as well as the endogenous Mis18BP1 protein (lower band) remained stable after 48 hours of Mis $18 \alpha$ and Mis $18 \beta$ depletion (Figure 3-3B).

Mis18 $\alpha$, Mis18 $\beta$ and Mis18BP1 protein levels are high during mitosis, but $\mathrm{Cdk} 1 / \mathrm{Cdk} 2$ activity inhibits the recruitment of Mis18BP1 to centromeres until G1 [163, 164]. The localization of Mis18BP1 requires the presence of Mis18 $\alpha$ and Mis18 $\beta$; therefore, one additional way in which Mis18 complex recruitment may be limited during mitosis would be to inhibit the interaction between the individual proteins outside of G1. In order to determine whether Mis18BP1, Mis18 $\alpha$ and Mis18 $\beta$ form a complex prior to 
CENP-A deposition, I generated chromatin-free extracts from mitotically arrested, GFPMis18 $\alpha$ expressing cells, and I.K. Nardi performed size exclusion chromatography on the extracts. Mis $18 \alpha$ and Mis $18 \beta$ eluted in the same peak fraction indicating the formation of a separate Mis18 $\alpha$-Mis18 $\beta$ complex present during mitosis (Figure 3-3D). In contrast, Mis18BP1 eluted as part of a larger molecular weight complex that was devoid of Mis18 $\alpha$ or Mis $18 \beta$. This suggests that Mis18BP1 does not associate with Mis $18 \alpha$ and Mis $18 \beta$ during mitosis, and regulated complex formation may be an additional mechanism by which a cell prevents the Mis 18 complex from prematurely associating with centromeres, prior to mitotic exit. 
Figure 3-3. Mis18BP1 exists in a separate complex from Mis18 $\alpha$ and Mis18 $\beta$ prior to G1.

(A) Representative images of HeLa Flp-In T-Rex cells stably expressing GFP-Mis18BP1 that were treated with either negative control siRNA or siRNA targeting Mis18 $\alpha$, Mis18 $\beta$ or Mis18BP1 for 48 hours. Centromeres were visualized using anti-CENP-T antibodies. Scale bar $=5 \mu \mathrm{m}$. (B) Whole cell lysates of cells treated as in A were blotted with polyclonal antibodies against Mis18 $\beta$ and Mis18BP1. Two bands are present in the Mis18BP1 immunoblot. The upper band corresponds to GFP-Mis18BP1, and the bottom band corresponds to the endogenous protein. A monoclonal anti-tubulin antibody was used as a loading control. (C) Graph shows the percentage of cells in which GFPMis18BP1 is localized to endogenous centromeres with respect to siRNA treatment. Double asterisks indicate a p-value of $<0.01$ with respect to the negative control siRNA condition, as calculated by the Student's t-test. (D) Mitotic extracts from cells stably expressing GFP-Mis18 $\alpha$ were run over a Superdex 200 size exclusion column. Fractions were blotted for the presence of Mis18BP1, GFP-Mis18 $\alpha$, or Mis18 $\beta$ in each fraction. Arrows highlight the migration of protein standards with the indicated Stokes radii. 
A

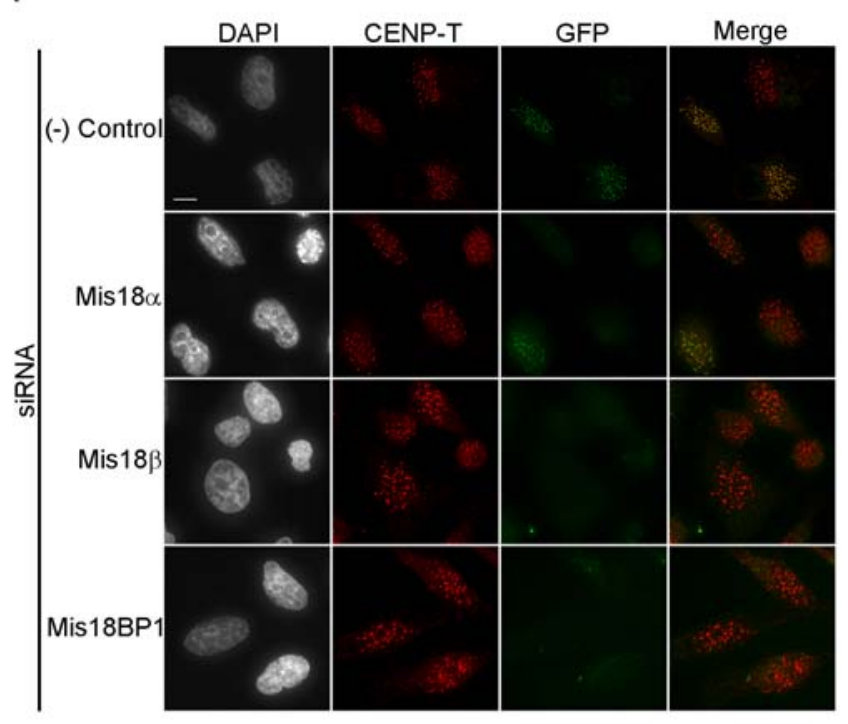

D

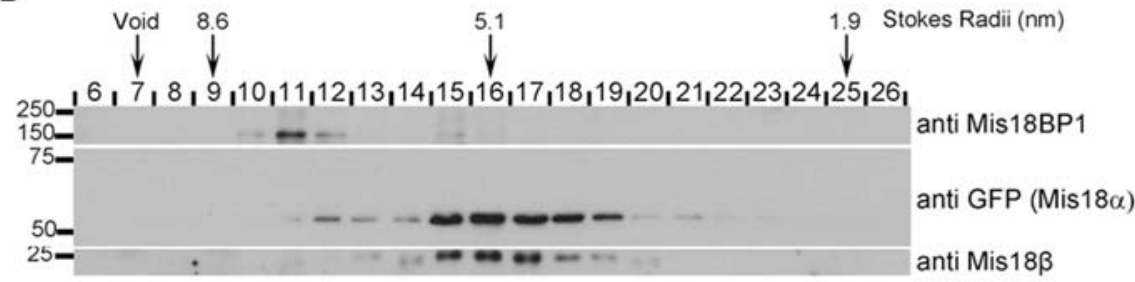

B
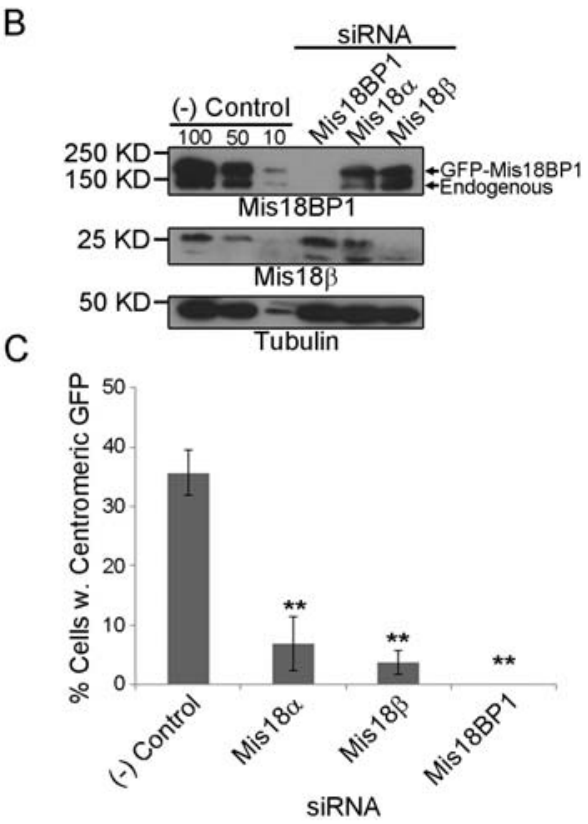

Figure 3-3

Includes data generated by I.K. Nardi 


\section{Mis18 $\alpha$ interacts directly with Mis18BP1 through the Yippee/Mis18}

\section{domain}

In order to gain a better understanding of the physical interactions that mediate Mis 18 complex formation, I.K. Nardi expressed the three Mis 18 complex proteins separately in bacteria and purified the recombinant proteins. Maltose binding protein (MBP) was fused to the N-terminus of full-length Mis18BP1. Both Mis18 $\alpha$ and Mis18 $\beta$ were N-terminally tagged with a dual tag consisting of a Strep-tag and the HA epitope (StrepHA). MBP-Mis18BP1 was incubated with StrepHA-Mis18 $\alpha$ and StrepHA-Mis18 $\beta$, either individually or together, and proteins bound to MBP-Mis18BP1 were isolated on amylose beads. When MBP-Mis18BP1 and StrepHA-Mis18 $\alpha$ were incubated together, MBP-Mis18BP1 bound to StrepHA-Mis18 $\alpha$ (Figure 3-4A, lane 4). However, MBPMis18BP1 was unable to pull down StrepHA-Mis18 $\beta$ unless StrepHA-Mis18 $\alpha$ was also present (Figure 3-4A, lane 5 and 6). Thus, Mis18BP1 directly interacts with Mis $18 \alpha$ but not Mis18 $\beta$, and these two highly similar proteins may have evolved to serve different functions in the CENP-A deposition pathway.

The Yippee/Mis18 domains present in Mis18 $\alpha$ and Mis18 $\beta$ each contain two highly conserved CXXC motifs, shown in the alignments generated by Geneious Pro software [90, 154, 163] (Figure 3-4B and Figure 3-5B). Replacing the first conserved cysteine with alanine (C85A) in Mis18 $\alpha$ eliminates its centromeric localization when transiently expressed in U2OS cells, without affecting steady-state levels of the protein (Figure 3-4, C and D), which is consistent with previous data [90]. I assessed the ability of Mis18BP1 to interact with wild type Mis18 $\alpha$ and Mis18 $\beta$ or Yippee/Mis18 domain 
mutants using the LacO interaction assay described above. Wild type and mutant constructs of Mis $18 \alpha$ or Mis $18 \beta$ were N-terminally tagged with mCherry-LacI and targeted to the LacO array as bait proteins. Full-length GFP-Mis18BP1 was co-expressed as the prey protein (Figure 3-4, E and F). Wild type mCherry-LacI-Mis18 $\alpha$ was able to recruit GFP-Mis18BP1 robustly to the LacO array, which is consistent with a direct interaction between Mis18 $\alpha$ and Mis18BP1. In contrast, Mis18BP1 recruitment was low when wild type mCherry-LacI-Mis18 $\beta$ was targeted to the LacO array (Figure 3-4G). This is consistent with Mis18 $\beta$ interacting with GFP-Mis18BP1 through endogenous Mis $18 \alpha$. Additionally, mutating the Yippee/Mis18 domain in Mis $18 \alpha$ by replacing the first cysteine of the domain with an alanine (mCherry-LacI-Mis $18 \alpha^{\mathrm{C} 85 \mathrm{~A}}$ ) failed to recruit GFP-Mis18BP1 in this assay. This indicates that the Yippee/Mis18 domain of Mis $18 \alpha$ is responsible for the interaction between Mis18 $\alpha$ and N-terminus of Mis18BP1. Previous experiments have shown that wild type Mis $18 \alpha$ cannot be recruited to centromeres in the absence of Mis18BP1, and the substitution of conserved cysteines within the Yippee/Mis 18 domain also disrupts Mis $18 \alpha$ centromeric localization [90]. Therefore, the in vitro pull downs and the $\mathrm{LacO}$ array experiments indicate that the inability of Mis $18 \alpha^{\mathrm{C} 85 \mathrm{~A}}$ to accumulate at centromeres is likely due to a disruption in its ability to bind Mis18BP1. 
Figure 3-4. Mis18 $\alpha$ interacts with Mis18BP1 through conserved cysteine residues.

(A) Immunoblots of amylose pull downs of recombinant MBP-Mis18BP1 incubated with StrepHA-Mis18 $\alpha$ or StrepHA-Mis18 $\beta$, alone or in combination using anti-HA or antiMis18 $\beta$ antibodies. (B) ClustalW2 alignment of Mis18 $\alpha$ homologs generated by Geneious Pro Software [154]. Darker highlighting and larger letter sizes indicate higher conservation. Break indicates a stretch of 39 amino acids, with respect to the human protein sequence, between the domain-specific cysteine repeats. The asterisk indicates the position of the cysteine to alanine mutant (C85A) in human Mis18 $\alpha$. (C) Anti-GFP immunoblot shows the expression of GFP-Mis18 $\alpha$ wild type or the C85A mutant in U2OS cells. Anti-tubulin blot was used as a loading control. (D) Representative images of the localization of either GFP-Mis18 $\alpha$ wild type or the C85A mutant. Centromeres are visualized by anti-CENP-C antibody. Insets highlight GFP localization to an individual centromere. For each condition, the percentage of cells with centromeric GFP signal, plus or minus the standard deviation across replicates, is shown in white. Scale bar $=5 \mu \mathrm{m}$. (E) Table summarizes the mCherry-LacI bait constructs and the GFP-Mis18BP1 prey construct used in F. (F) Representative images of mCherry-LacI-Mis18 $\alpha$ or -Mis $18 \beta$ constructs targeted to the LacO array and the recruitment of full-length GFP-Mis18BP1. Insets highlight the array. Scale bar $=5 \mu \mathrm{m}$. (G) Graph indicates the recruitment of GFPMis18BP1 to the array, over the average nuclear GFP background signal, when either mCherry-LacI or mCherry-LacI-Mis18 $\alpha$ or -Mis18 $\beta$ were used as bait. Error bars describe the standard deviation across replicates. Double asterisks indicate a p-value of $<$ 
0.01 with respect to the recruitment of GFP-Mis 18BP1 by mCherry-LacI alone, as calculated by the Student's t-test.

A

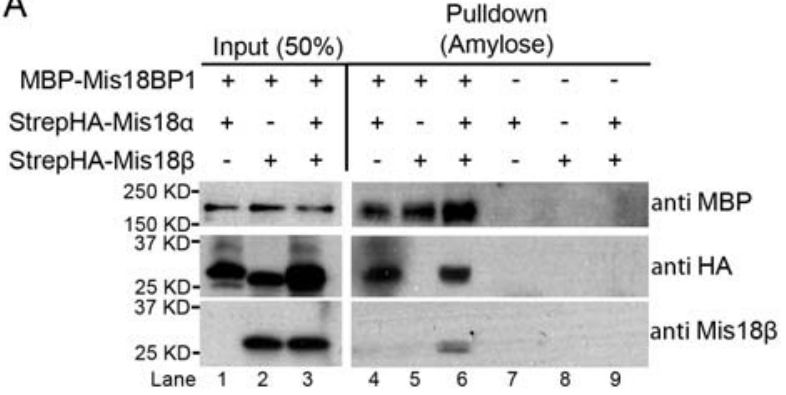

B
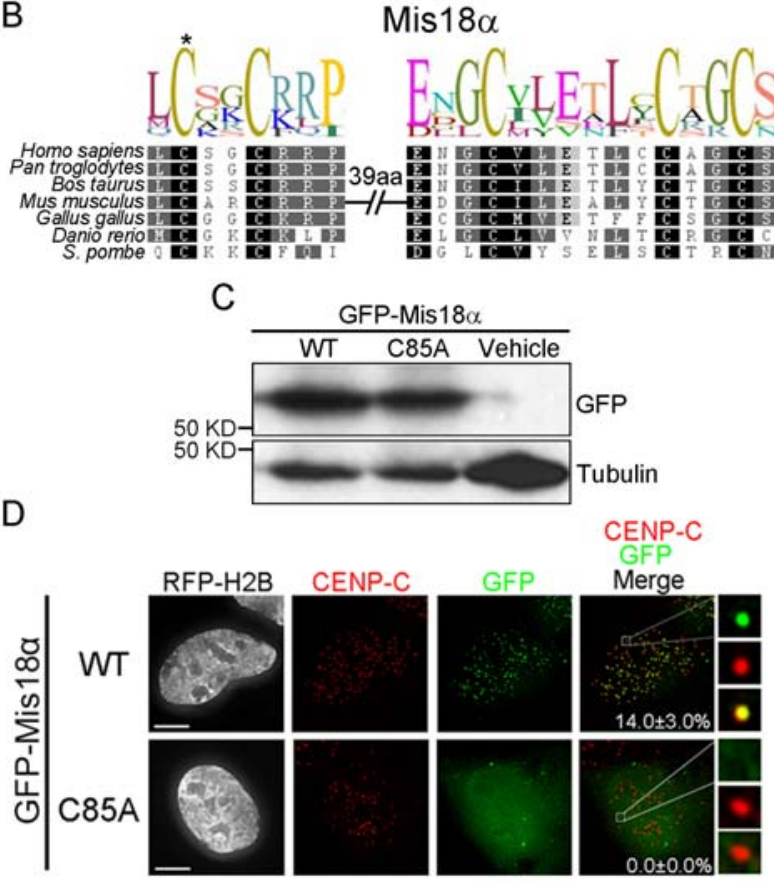

E
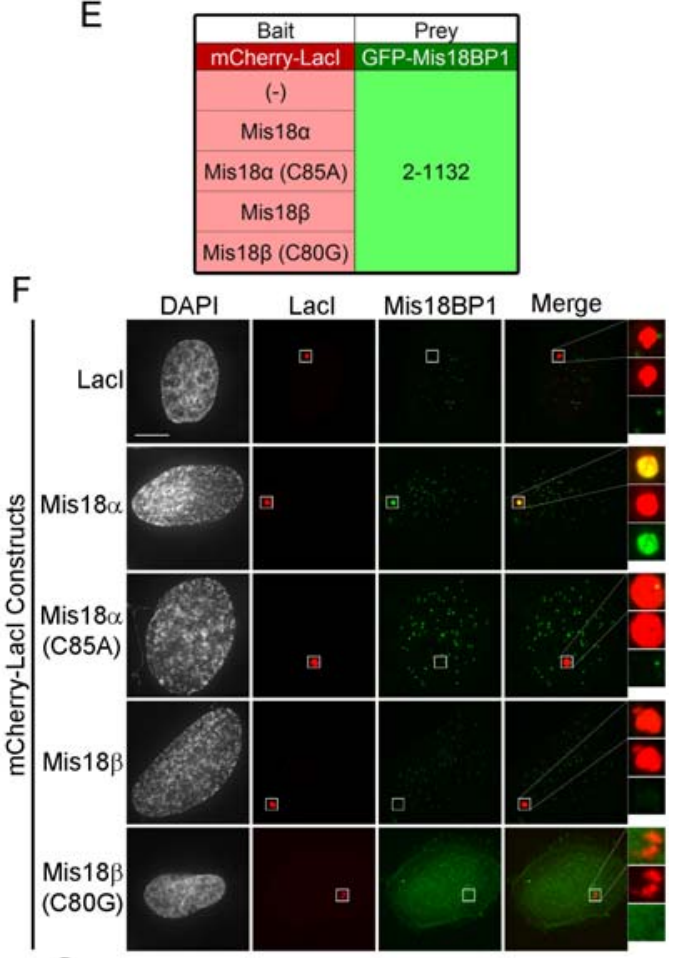

G

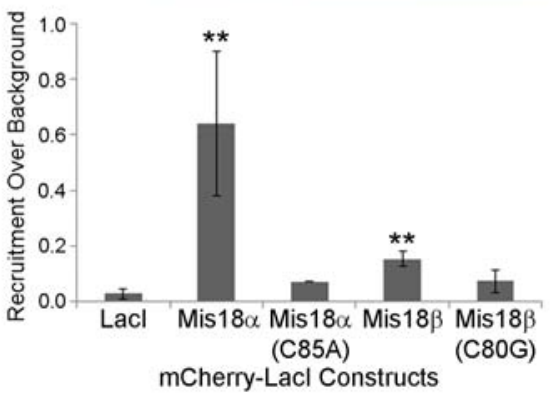

Figure 3-4

Panel A, C and D include data generated by I.K. Nardi 


\section{Mis18 $\beta$ binds CENP-C in a cell cycle dependent manner}

Depleting CENP-C, a constitutive centromere protein, in mouse cells by shRNA or by antibody depletion in Xenopus extracts reduces the centromeric localization of Mis18BP1, which suggests an important role for CENP-C in the recruitment of the Mis18 complex $[99,100]$. It is also clear that Mis $18 \alpha$ and Mis $18 \beta$ play a significant role in centromeric recruitment of the Mis18BP1 constructs studied in Figure 3-1. Therefore, I asked whether the Mis18 $\alpha$ and Mis18 $\beta$ proteins contribute to the recognition of CENP-C by the Mis 18 complex. Work in mouse embryonic stem cells has shown that Mis18 $\alpha$ can immunoprecipitated CENP-C, but it is not known if the interaction is direct or mediated through other members of the complex or CCAN [109]. We identified a conserved 250 amino acid region of human CENP-C spanning amino acids 694 to 943 based on the Mis18BP1 binding domain within Xenopus and mouse CENP-C homologs (CENP-C ${ }^{694-}$

${ }^{943}$ ). This region included the previously defined CENP-C centromere-targeting domain [168-171].

I.K. Nardi generated an N-terminal fusion of this CENP-C fragment to a dual tag consisting of a 6xHis-tag and NusA (HisNusA) and expressed it in bacteria. The recombinant HisNusA-CENP-C ${ }^{694-943}$ protein was incubated with StrepHA-Mis $18 \alpha$ or StrepHA-Mis $18 \beta$ alone or in combination. Proteins that bound CENP-C were isolated by Ni-NTA agarose pull down (Figure 3-5A). StrepHA-Mis18 $\beta$ was able to co-purify with HisNusA-CENP-C ${ }^{694-943}$ while Mis $18 \alpha$ failed to interact with HisNusA-CENP-C $C^{694-943}$ when incubated alone (Figure 3-5A, Lanes 4 and 5). However, incubating both Mis18a and Mis $18 \beta$ proteins with CENP-C ${ }^{694-943}$ resulted in both being found in the pull down 
fraction (Figure 3-5A, Lane 6). This is indicated by the doublet band in the HA immunoblot, highlighted by the small black arrows to the left of the blot, as StrepHAMis18a is slightly larger than StrepHA-Mis18ß.

The Yippee/Mis18 domain of Mis18 $\alpha$ is known to contribute to its centromeric localization; however, the function of a similar domain in Mis18 $\beta$ is unknown [90] (Figure 3-5B). In order to determine whether the centromeric localization of Mis18 $\beta$ also depends on this domain, I.K. Nardi compared the expression and localization of wild type GFP-tagged Mis $18 \beta$ and a GFP-Mis $18 \beta$ construct containing a cysteine to glycine substitution for the first cysteine in the domain (C80G) (Figure 3-5, C and D). Both constructs were transiently transfected into U2OS cells and were expressed at similar levels. Wild-type GFP-Mis $18 \beta$ localized to centromeres in $6.0 \pm 1.0 \%$ of transfected cells, whereas GFP-Mis $18 \beta^{\mathrm{C} 80 \mathrm{G}}$ was not recruited to endogenous centromeres.

In order to determine whether the loss of centromeric localization was related to an altered ability of Mis $18 \beta^{\mathrm{C} 80 \mathrm{G}}$ to interact with CENP-C, I assayed the interactions between these proteins by using the LacO array. As a bait protein, mCherry-LacI-CENP$\mathrm{C}^{694-943}$ was targeted to the array, and the recruitment of wild type or mutant GFP-Mis18 $\beta$ to the array was analyzed (Figure 3-5, E- H). I observed that mCherry-LacI-CENP-C ${ }^{694-}$ ${ }^{943}$ was able to recruit wild type GFP-Mis $18 \beta$ to the array, which is in agreement with the in vitro pull down results in Figure 3-5A. Similar to the elimination of centromeric recruitment, the Yippee/Mis 18 mutant (GFP-Mis18${ }^{\mathrm{C} 80 \mathrm{G}}$ ) was unable to interact with mCherry-LacI-CENP-C ${ }^{694-943}$ at the array. 
In addition, I observed that the interaction between CENP-C ${ }^{694-943}$ and wild type GFP-Mis $18 \beta$ at the LacO array appeared to be cell cycle regulated. Recruitment of GFPMis $18 \beta$ to the CENP-C bound LacO array occurred preferentially in cells that were in G1, which was indicated by the presence of GFP-Mis18 $\beta$ at endogenous centromeres (Figure 3-5H). GFP-Mis18 $\beta$ localized to the mCherry-LacI-CENP-C ${ }^{694-943}$ containing arrays in $80.8 \%$ of cells in which GFP-Mis $18 \beta$ was present at endogenous centromeres. Whereas, in those cells without GFP-Mis $18 \beta$ localized to centromeres, only $6.3 \%$ of cells recruited GFP-Mis18 $\beta$ to CENP-C occupied LacO arrays. 
Figure 3-5. Mis18 $\beta$ binds CENP-C in a cell cycle dependent manner to generate full centromeric recruitment.

(A) Ni-NTA pull down of HisNusA-CENP-C ${ }^{694-943}$ incubated with StrepHA-Mis18 $\alpha$ or StrepHA-Mis18ß, alone or in combination, and immunoblotted with anti-NusA, anti-HA or anti-Mis $18 \beta$ antibodies. (B) ClustalW2 alignment of Mis18 $\beta$ homologs generated by Geneious Pro software [154]. Darker highlighting and larger letter sizes indicate more highly conserved residues. Break indicates a stretch of 47 amino acids, with respect to the human protein sequence, between the domain specific cysteine repeats. Asterisk indicates the cysteine to glycine substitution (C80G). (C) Immunoblot showing the expression of wild type GFP-Mis18 $\beta$ or the C80G mutant in U2OS cells. Anti-tubulin blot was used as a loading control. (D) Representative images of the localization of either GFP-Mis18 $\beta$ wild type or the C80G mutant in U2OS cells. Centromeres are visualized by an antiCENP-C antibody. Insets highlight GFP localization to an individual centromere. For each condition, the percentage of cells with centromeric GFP signal, plus or minus the standard deviation across replicates, is shown in white. Scale bar $=5 \mu \mathrm{m}$. (E) Table of mCherry-LacI and GFP-tagged constructs used in F. (F) Representative images of targeting mCherry-LacI alone or mCherry-LacI-CENP-C ${ }^{694-943}$ to the LacO array and assessing the recruitment of wild type GFP-Mis18ß or GFP-Mis $18 \beta^{\mathrm{C} 80 \mathrm{G}}$. (G) Graph indicates the recruitment of wild type GFP-Mis $18 \beta$ or GFP-Mis $18 \beta^{\mathrm{C} 80 \mathrm{G}}$ to the array, over the average nuclear background GFP signal. Error bars describe the standard deviation across replicates. Triple asterisks indicate a p-value of $<0.001$ with respect to the 
recruitment of wild type GFP-Mis $18 \beta$ by mCherry-LacI alone, as calculated by the Student's t-test.

A

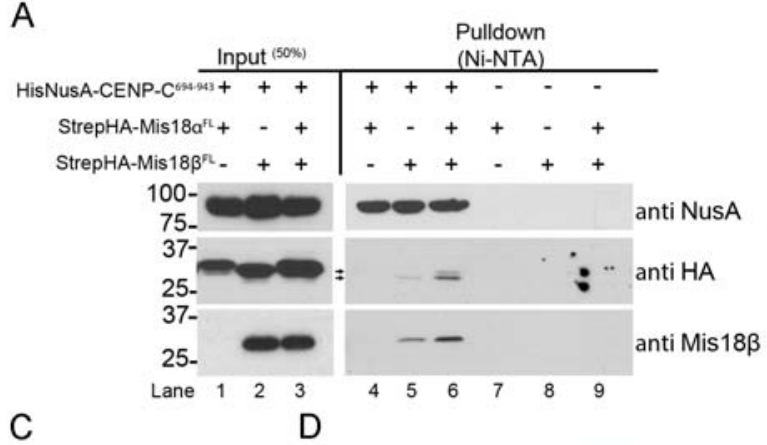

C
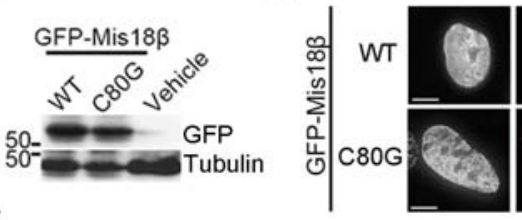

$\mathrm{F}$

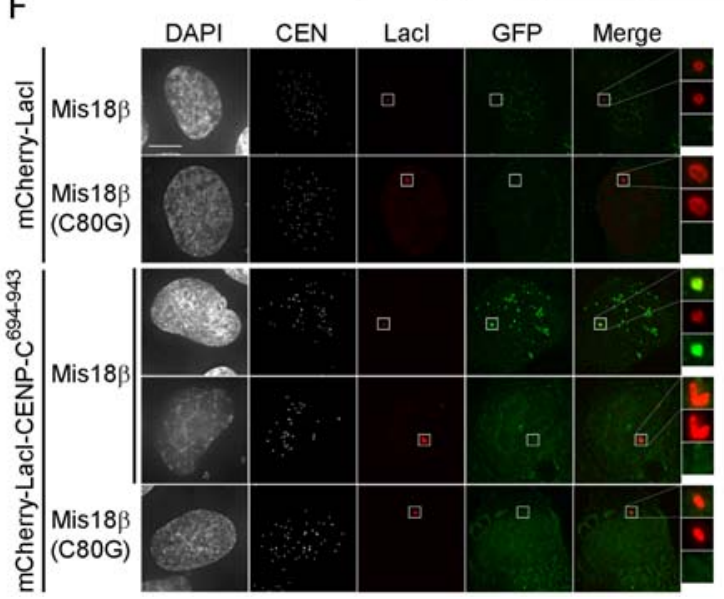

B

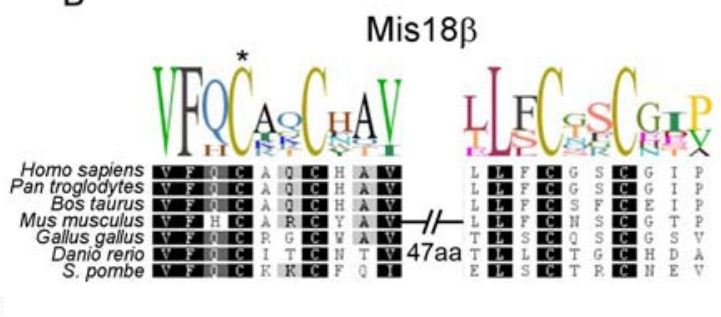

$\mathrm{E}$

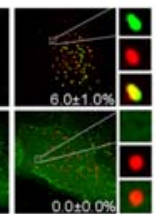

Bait mCherry-Lacl GFP-Mis18BP1

\begin{tabular}{c|c}
$(-)$ & Mis18 $\beta$ WT \\
\hline CENP-C $^{694-943}$ & Mis18 $\beta$ (C80G)
\end{tabular}
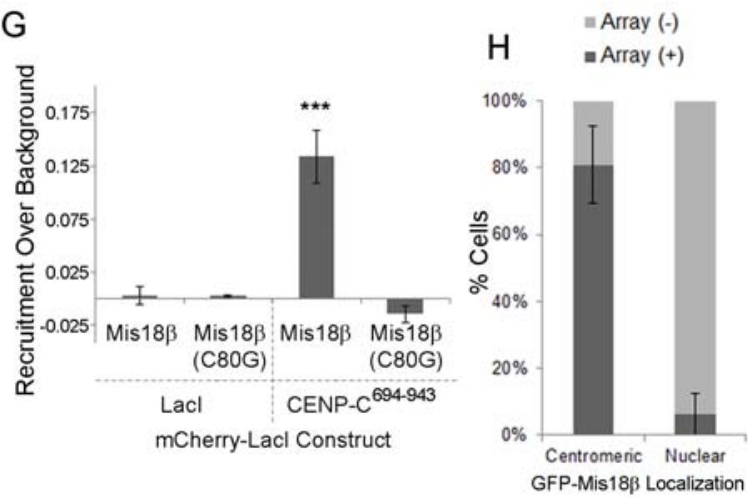

Figure 3-5

Panels $A, C$ and D include data generated by I.K. Nardi 


\section{The CENP-C-Mis18ß interaction is regulated by kinase activity}

In the above figure the recruitment of GFP-Mis $18 \beta$ to mCherry-LacI-CENP-C ${ }^{694-}$ ${ }^{943}$ targeted LacO arrays appeared to be cell cycle regulated. If a cell had GFP-Mis18 $\beta$ at centromeres, it was more likely to have GFP-Mis $18 \beta$ localized to the array. Since Mis $18 \mathrm{BP} 1$ recruitment is mediated by $\mathrm{Cdk} 1 / \mathrm{Cdk} 2$ activity, I hypothesized that the Mis18ß-CENP-C interaction is also regulated by kinase activity. Cdk activity is high when Mis18BP1 is actively prohibited from localizing to centromeric chromatin. Pololike kinase $1(\mathrm{Plk} 1)$ is another mitotic kinase that has many roles in ensuring proper cell cycle progression and cell division [172].

In order to test the hypothesis that the Mis18 $\beta$-CENP-C interaction is also regulated by kinase activity, I targeted mCherry-LacI-CENP-C $\mathrm{C}^{694-943}$ to the array and coexpressed GFP-Mis $18 \beta$. One hour prior to fixation and staining, I treated the cells with either the Cdk inhibitor, purvalanol A (PurA), or the Plk1 inhibitor (BI 2536). I then stained the cells for endogenous centromeres and assessed the centromeric localization pattern and array recruitment of GFP-Mis $18 \beta$.

In cells treated with DMSO as a negative control, the cells behaved similarly to the untreated cells in Figure 3-5H. If a cell recruited GFP-Mis $18 \beta$ to centromeres, then that cell was also likely to have recruited GFP-Mis $18 \beta$ to the mCherry-LacI-CENP-C ${ }^{694-}$ ${ }^{943}$ targeted array (Figure 3-6, A and B). Cells that did not recruit GFP-Mis $18 \beta$ to endogenous centromeres were unlikely to have GFP-Mis $18 \beta$ at the mCherry-LacI-CENP$\mathrm{C}^{694-943}$ arrays. Treating cells with a Cdk inhibitor also had little effect on the correlated pattern of recruitment to centromeres and to the array. However, when cells were treated 
with an inhibitor to Plk1, the number of non-centromeric GFP-Mis $18 \beta$ cells that recruited GFP signal to the array increased. This indicates that Plk1 may ensure that Mis $18 \beta$ and CENP-C do not interact prior to G1, similarly to the effect of Cdk1/Cdk2 on Mis18BP1. I wanted to determine if Mis18 $\beta$ was likely phosphorylated in mitosis, similar to Mis18BP1 discussed in chapter 2. HeLa cells were either allowed to randomly cycle, or else were blocked in nocodazole overnight. In addition, a proportion of nocodazole treated cells were released from mitosis and allowed to reenter the cell cycle for 3 hours before harvesting. Whole cell lysates were generated from each condition and analyzed by western blot for changes in apparent molecular weight (Figure 3-6C). In lysates from randomly cycling cells, the major band that was recognized by the Mis $18 \beta$ antibody was around $22 \mathrm{KD}$. In the mitotically arrested cells, the predominant band was shifted to around $26 \mathrm{KD}$. This is very similar to what was seen for Mis18BP1. Interestingly, in the nocodazole release lysates, both the $22 \mathrm{KD}$ and $26 \mathrm{KD}$ bands are present, albeit in much lower amounts. This suggests that Mis $18 \beta$ is post-translationally modified in mitosis, and this mark is removed as cells exit mitosis and progress into G1.

These data indicate that Plk1, or another mitotic kinase, may phosphorylate Mis18 $\beta$ in mitosis in order to regulate its binding affinity to the centromere, through CENP-C. Therefore, I wanted to assess whether Plk1 can physically interact with the Mis18 complex in vivo. I targeted mCherry-LacI-tagged Mis18 $\alpha$, Mis18 $\beta$ and Mis18BP1 to the array and coexpressed GFP-Plk1 (Figure 3-6, D and E). I could see Plk1 localize I saw recruitment of Plk1 to the Mis 18 targeted arrays between $50 \%$ and $95 \%$ of the time, depending on the construct. While much of this preliminary data is correlative, it agrees 
with recent data that declares the Mis $18 \beta$ is a substrate for Plk1 in vivo [173]. These observations open several lines of future inquiry into additional cell cycle regulation mechanisms underlying the recruitment of Mis18 complex to centromeres. 
Figure 3-6. The CENP-C-Mis18 $\beta$ interaction is regulated by kinase activity.

(A) Representative images of U2OS-LacO cells cotransfected with mCherry-LacI-CENPC- ${ }^{694-943}$ and GFP-Mis18 8 . Transfected cells were then treated with DMSO, purvalanol A (PurA) or BI 2536 for $1 \mathrm{~h}$. Boxes and enlarged regions highlight colocalization between CENP-C and Mis $18 \beta$ at the array. Scale bar $=5 \mu \mathrm{m}$. (B) Graph depicts the percentage of cells with Mis $18 \beta$ at centromeres $(\mathrm{CEN}+)$ or not (CEN-) that recruit GFP-Mis $18 \beta$ to the array with respect to inhibitor treatment. Error bars show the standard deviation between replicates. (C) Anti-Mis $18 \beta$ western blot of whole cell lysates from HeLa cells that were allowed to randomly cycle, were blocked in mitosis or were blocked in mitosis and released for $3 \mathrm{~h}$. Arrows highlight the two different molecular weight bands. (D)

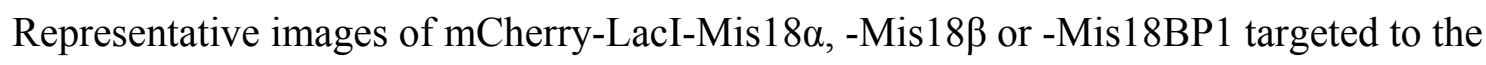
array with GFP-P1k1 coexpressed. Boxes and enlarged sections highlight colocalization between the Mis18 proteins and Plk1 at the array. Scale bar $=5 \mu \mathrm{m}$. (E) Graph depicting the percentage of cotransfected cells that recruited GFP-Plk1 to the array. 

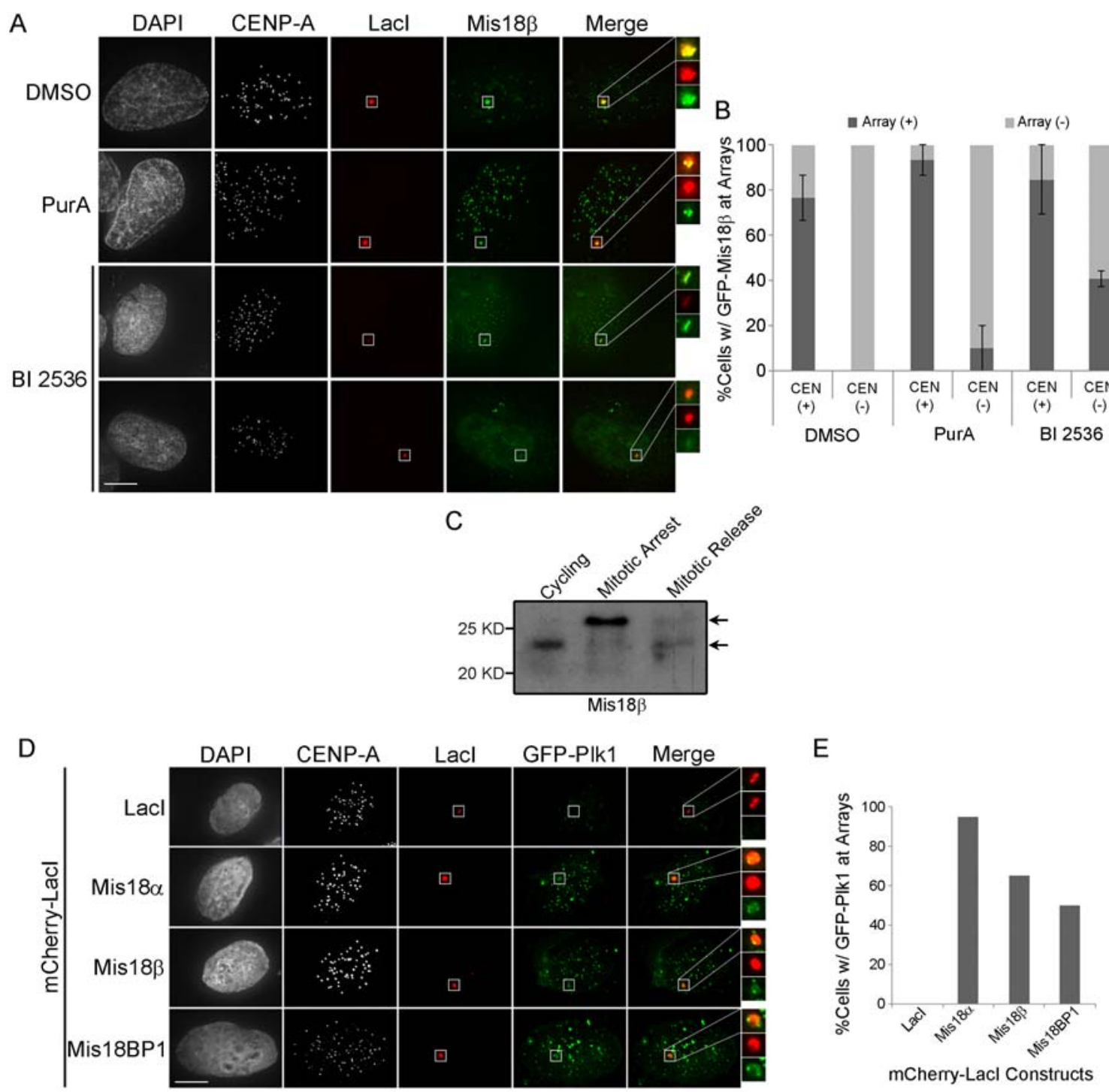

Figure 3-6 


\section{Full recruitment of the Mis 18 complex by CENP-C requires multiple interactions}

The central region of Mis18BP1 (Mis18BP $1^{476-721}$ ) has been previously reported to bind CENP-C; however, this region of Mis18BP1 is not sufficient to localize to centromeres (Figure 3-1, C and D) [99, 100]. Therefore, we assayed whether the ability of Mis18BP1 to be recruited by CENP-C on chromatin is influenced by the amino terminus of Mis 18BP1. Using the LacO system, mCherry-LacI-CENP-C ${ }^{694-943}$ robustly recruited GFP-tagged full-length Mis18BP1 to the array (Supplemental Figure S3-3, A and B). The amino-terminal fragment, GFP-Mis18BP1 $1^{2-721}$, was also efficiently recruited to the CENP-C containing arrays, similar to full-length Mis18BP1. This is the same fragment that was sufficient to support centromere recruitment (Figure 3-1C).

Mis18BP $1^{476-721}$ was recruited to the LacO array by CENP-C, but to a significantly lesser degree than the full-length or Mis18BP1 $1^{2-721}$ construct (Supplemental Figure S3-3B). Therefore, although the central region of Mis18BP1 (Mis18BP $1^{476-721}$ ) is able to interact with the C-terminal portion of CENP-C, Mis18BP1 requires its amino-terminus to provide the most efficient recognition of CENP-C through an interaction with Mis $18 \alpha$ and Mis18 $\beta$. 


\section{Discussion}

The stable inheritance of centromeric chromatin is dependent on the Mis 18 complex, which includes Mis18 $\alpha$, Mis18 $\beta$ and Mis18BP1. The recruitment of new CENP-A and its assembly factor, HJURP, requires the centromeric localization of the Mis 18 complex. Therefore, Mis18 recruitment is a defining step in the early stages of centromere specification. Here we demonstrate that despite their common origin, the Mis18 $\alpha$ and Mis18 $\beta$ paralogs participate in different interactions within the Mis 18 and CCAN complexes. Mis $18 \alpha$ binds directly to the amino-terminus of Mis18BP1, while Mis18 $\beta$ interacts with centromere targeting domain of CENP-C. The interaction between Mis $18 \beta$ and CENP-C is restricted in the cell cycle and contributes to the G1 specific recruitment of the Mis 18 complex to centromeres.

Mis18BP1 contains two highly conserved domains, the SANT and SANTassociated (SANTA) domains, which are found in Mis18BP1 proteins from humans to C. elegans. We observed that fragments of human Mis18BP1 that lacked either SANT or SANTA domains were efficiently recruited to endogenous centromeres. Therefore, neither the SANT nor SANTA domains contribute to the centromere localization of the complex in humans. This is consistent with work with the Arabidopsis KNL-2, homolog, which shows that the SANTA domain is not required for localization of KNL-2 to centromeric regions [165]. Previous reports stated that the SANT domain was required to confer CENP-C binding in mice; however, this domain is dispensable in human cells. In addition, neither domain mediates the interaction with known centromere assembly 
factors such as Mis18 $\alpha$ and Mis18 8 . Therefore, the function of these conserved domains remains to be determined.

Mis18BP1 was previously shown to interact with the CCAN protein, CENP-C, in Xenopus and mouse models, which has been proposed a possible centromere-specific targeting mechanism for the Mis18 complex [99, 100]. Our data demonstrate that while the CENP-C binding domain of Mis18BP1 is required for recruitment of the complex, it alone is not sufficient to recruit Mis18BP1 to centromeres. We show that centromere recruitment of Mis18BP1 requires its amino terminus in addition to the previously defined CENP-C binding domain. The required amino-terminus interacts directly with Mis18 $\alpha$. In turn, Mis18 $\beta$ helps facilitate a robust interaction with CENP-C.

While the cell cycle regulation mechanism behind Mis18BP1 localization has been previously determined, our data indicate additional mechanism that tie Mis 18 complex localization and function to the cell cycle. It appears that the interaction between CENP-C and Mis $18 \beta$ is regulated by the cell cycle. Preliminary data (Figure 3-6) and data generated in other laboratories suggests that polo-like kinase 1 may play a significant role in regulating this interaction [173]. However, at this point, the majority of the data remains correlative. We have looked at the primary sequence of Mis $18 \beta$ and have identified a putative Plk1 site. Phospho mutants, such as the alanine substitution mutant that we have recently generated, will allow us to study this interaction more robustly. Therefore, the Mis 18 complex requires that Mis18BP1 and Mis18 $\beta$ both bind CENP-C, being bridged by Mis18 $\alpha$, in order to generate a stable and cell cycle regulated interaction between the Mis18 complex and the CCAN. 
Different higher eukaryotes use somewhat distinct mechanisms to achieve centromere inheritance that include a partially overlapping set of proteins. The Mis 18 and HJURP proteins are conserved in fission yeast and humans, as well as a wide variety of eukaryotes, but have not been found in C. elegans and insects. C. elegans possess a Mis18BP1 homolog [92]. CAL1 in flies acts as a functional homolog of HJURP, despite a lack of sequence similarity, but nevertheless it depends on CENP-C for proper recruitment to existing centromeres $[94,95,124,174]$. Similar to humans, many organisms in which both Mis18 and Mis18BP1/KNL2 homologs have been identified, two Mis18 paralogs have also identified. This is consistent with a conserved separation of function between the two Mis18 paralogs that we have demonstrated here in human cells. Mis $18 \alpha$ and Mis $18 \beta$ paralogs share a conserved Yippee/Mis18 domain. Previously, mutations altering the conserved cysteine residues within the two CXXC motifs of the Yippee/Mis18 domain of Mis18 $\alpha$ were shown to eliminate centromere recruitment of the Mis 18 complex [90]. We show that the loss of centromere localization in Mis18a Yippee mutants is due to the inability of the mutant to bind Mis18BP1. Interestingly, mutating any of the four conserved cysteines at positions $85,88,141$ and 144 leads to centromere mis-localization, which suggests that each of these cysteines contributes to a common structure rather than directly mediating the interaction with Mis18BP1 [90, 163]. Similarly, replacing of one of the conserved cysteines in Mis18 $\beta$ with glycine (Mis18${ }^{\mathrm{C} 80 \mathrm{G}}$ ) also leads to a loss of centromere recruitment (Figure 3-5). This is due to the inability of the mutant Mis $18 \beta$ to bind CENP-C. While the Mis $18 \alpha$ and Mis $18 \beta$ paralogs use their Yippee/Mis18 domains to interact with different partners, the 
integrity of both domains in Mis18 $\alpha$ and Mis18 8 are crucial to mediate the multiple interactions required for complete recruitment of the Mis18 complex to centromeres.

Mis18 proteins have been previously thought to form a constitutive three-protein complex. Our data demonstrate that the formation of the three-protein Mis18 $\alpha$, Mis18 $\beta$ and Mis18BP1 complex is a regulated event. Mis18 $\alpha$ and Mis18 $\beta$ exist in a separate complex from Mis 18BP1 during mitosis, at a time when the cell is poised to assemble new centromeric nucleosomes, but is inhibited from doing so by high Cdk activity [164] (Figure 3-7). As evidenced by our deletion mutant analysis, Mis 18BP1 that is unable to bind to Mis $18 \alpha$ and Mis18 $\beta$ is not recruited to centromeres. Likewise, Mis18BP1 cannot be recruited to centromeres when Mis18 $\alpha$ or Mis $18 \beta$ are depleted, even though Mis18BP1 protein levels remain high. Full assembly of the three-protein Mis18 complex may only occur at chromatin in G1 following reduction of Cdk activity, ensuring proper regulation of CENP-A deposition. 
Figure 3-7. The roles of the Mis18 $\alpha$ and Mis18 $\beta$ paralogs in centromere specification.

This model depicts the manner by which the Mis18 complex interacts with the human centromere in a cell cycle dependent manner. Throughout mitosis, Cdk activity is high and the Mis 18 complex is prevented from associating with centromeric chromatin. In addition, Mis18 $\alpha$ and Mis18 $\beta$ do not interact with Mis18BP1. Therefore, the components of the Mis18 complex reside in separate complexes, and both Mis18BP1 and Mis18 $\beta$ are unable to associate with CENP-C. Without the localization of the Mis18 complex, HJURP is maintained off of centromeric chromatin and CENP-A deposition does not occur. After mitotic exit, Cdk activity levels drop, which allows the Mis18 complex to form and localize to the centromere. Finally, the Mis18 complex recruits HJURP to the centromere and CENP-A is deposited in G1.

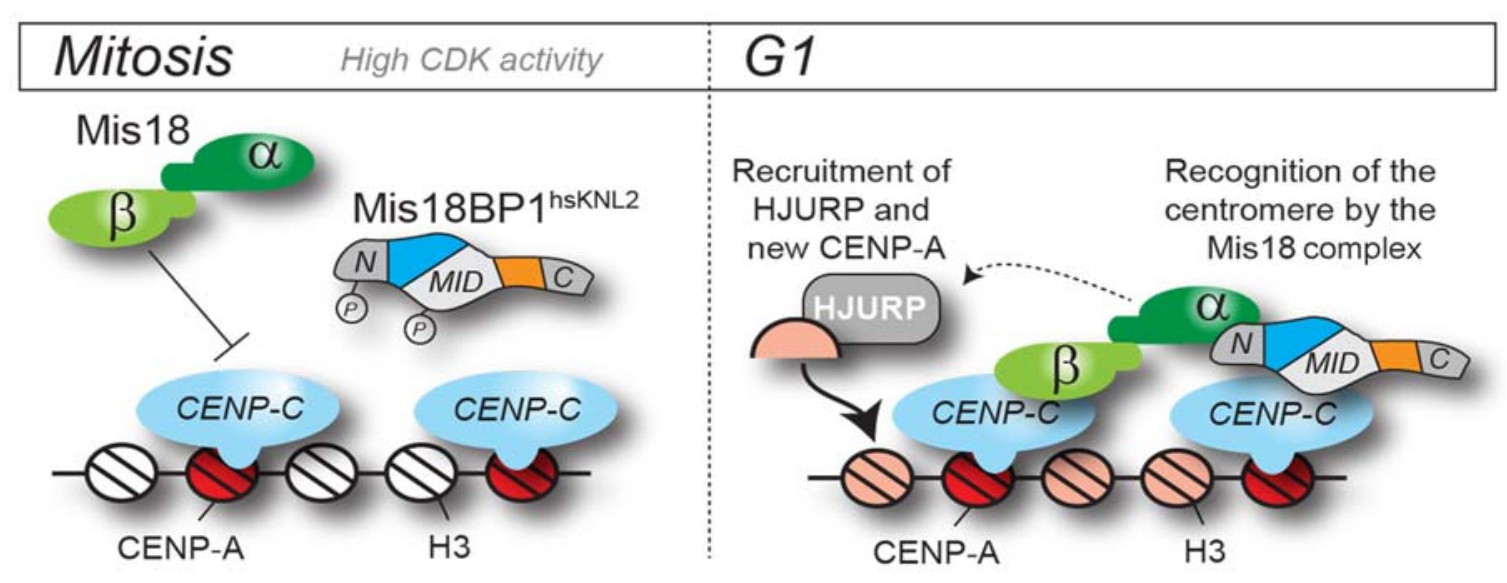

Figure 3-7 


\section{Materials and Methods}

\section{Indirect immunofluorescence and image acquisition}

Cell lines were grown on poly-lysine coated glass coverslips, fixed for $10 \mathrm{~min}$ with $37^{\circ} \mathrm{C} 4 \%$ formaldehyde in PBS $\left(137 \mathrm{mM} \mathrm{NaCl}, 2.7 \mathrm{mM} \mathrm{KCl}, 5.4 \mathrm{mM} \mathrm{Na}_{2} \mathrm{HPO}_{4}, 1.8\right.$ $\mathrm{mM} \mathrm{KH}_{2} \mathrm{PO}_{4}$ ) and then quenched for $5 \mathrm{~min}$ with $100 \mathrm{mM}$ Tris $\mathrm{pH}$ 7.5. Coverslips were blocked for $1 \mathrm{~h}$ in blocking buffer: $2 \%$ heat inactivated FBS, $2 \%$ BSA, and $0.1 \%$ TritonX100 in PBS. Primary antibodies were incubated for $1 \mathrm{~h}$ in blocking buffer: anti-CENPA mouse monoclonal antibody (ab13939, Abcam) at $1 \mu \mathrm{g} / \mathrm{mL}$ and anti-CENP-T rabbit polyclonal antibody serum (D. Cleveland, Ludwig Institute for Cancer Research) at 1:2000. Coverslips were washed three times for 5 min with $0.1 \%$ Triton-X100 in PBS. Secondary antibodies were diluted in blocking buffer and were incubated with the coverslips for $1 \mathrm{~h}$ (Alexa-647 conjugated goat-anti-mouse at $0.375 \mu \mathrm{g} / \mathrm{mL}$, Cy5 conjugated goat-anti-rabbit conjugated at $0.375 \mu \mathrm{g} / \mathrm{mL}, \mathrm{Cy} 3$ conjugated goat-anti-mouse at $0.75 \mu \mathrm{g} / \mathrm{mL}$; Jackson ImmunoResearch Laboratories, Inc.). Cells were washed as above and then incubated in PBS containing $0.2 \mu \mathrm{g} / \mathrm{mL}$ of DAPI for $5 \mathrm{~min}$. Coverslips were rinsed with PBS prior to mounting onto glass slides with Prolong Gold Antifade Reagent (Life Technologies).

Fluorescence microscopy images were acquired using a DeltaVision deconvolution microscope (GE Healthcare Lifesciences) equipped with a CoolSNAP $\mathrm{HQ}^{2}$ camera (Photometrics). Images were collected using either a 60X (numerical aperture $=1.42$; Olympus) or $100 \mathrm{X}$ (numerical aperture $=1.40$; Olympus) oil immersion lens. All images within an experiment were acquired using identical exposure times 
across all conditions. Images shown in figure panels are deconvolved maximum projections and scaled equally with the exception of Figure 1A where GFP-

Mis18BP1 $1^{\Delta 377-475}$ transfected cells were stained with the CENP-A monoclonal antibody, instead of CENP-T, and imaged and scaled separately from the rest of the panel.

\section{Image quantitation}

CENP-A intensity at centromeres was measured from non-deconvolved maximum projections, using ImageJ [175]. The background intensity for an individual nucleus was determined by averaging the integrated intensity of at least three non-centromeric locations within the nucleus. The integrated intensity of at least five random centromeres in each nucleus was measured, and the average background for that nucleus was subtracted from each measurement. Background-corrected centromere intensities were then averaged across each condition. Standard deviations were calculated between the averaged centromere intensities for each condition, across replicates.

The intensities of GFP bait proteins recruited to the LacO arrays were measured from non-deconvolved maximum projections using ImageJ. To determine the enrichment of GFP at the array the "recruitment over background" was calculated as follows. The background intensity of an individual nucleus was determined by averaging the integrated intensity of three non-centromeric, non-array containing locations within the nucleus. The integrated intensity at each array was then divided by the average background intensity within the individual cell to generate a ratio of the GFP signal at the array over the average GFP background intensity of the entire nucleus. An integrated 
intensity at the array, equal to that of the nuclear background, yielded a ratio equal to one, which was subtracted from all calculated ratios to give a level of recruitment above the nuclear background signal. Standard deviations between replicates were calculated based on the average of these corrected ratios for each condition.

\section{Tissue culture}

HeLa derived cells lines were cultured in DMEM High Glucose (Life Technologies) supplemented with 10\% heat inactivated FBS (Optima, Atlanta Biologicals) and 1X Penicillin/Streptomycin (Life Technologies). U2OS derived cell lines were cultured in DMEM High Glucose GlutaMAX ${ }^{\mathrm{TM}}$ (Life Technologies) supplemented with 10\% FBS and 1X Penicillin/Streptomycin. Cells were incubated at $37^{\circ} \mathrm{C}$ in $5 \% \mathrm{CO}_{2}$ and $85 \%$ humidity. A stable GFP-Mis $18 \mathrm{BP} 1$ expressing cell line was generated using the Flp-In system (Life Technologies) in a previously established HeLa T-REX Flp-In cell lines (S. Taylor, University of Manchester). Recombined cell lines were maintained in $200 \mu \mathrm{g} / \mathrm{mL}$ Hygromycin B-supplemented media.

\section{siRNA and plasmid transfections}

U2OS cells were plated at a density of either 10,400 cells $/ \mathrm{cm}^{2}$ or 35,000 cells $/ \mathrm{cm}^{2}$ in a 6-well plate or 24-well plate respectively and allowed to attach to the plate and coverslips for DNA transfections. The following day, cells were transfected with either 1 $\mu \mathrm{g}$ plasmid DNA (6-well format) or with $300 \mathrm{ng}$ (24-well format) using Lipofectamine 2000 in Opti-MEM according the manufacturer's protocol (Life Technologies). For 
centromere localization counts of the GFP-tagged Mis18BP1 fragments or the GFPMis18 $\alpha$ and GFP-Mis18 $\beta$ constructs, cells were transfected with a 10:1 ratio of GFP construct to RFP-H2B plasmid, which served as a transfection marker. For LacO array experiments, mCherry-LacI constructs were always transfected in a 1:2 ratio with the GFP bait proteins. The transfection media was removed after $10-12 \mathrm{~h}$ and replaced with fresh growth media. Cells were harvested with 3mM EDTA-PBS for western blot analysis or fixed for immunofluorescence $48 \mathrm{~h}$ post-transfection.

For siRNA depletion and rescue experiments, U2OS cells were plated at a density of 10,400 cells $/ \mathrm{cm}^{2}$ in a 6 -well plate. The following day cells were transfected with Lipofectamine 2000 in Opti-MEM according to the manufacturer's directions, including 30 pmol Silencer® Select siRNA (Mis18BP1 5'-GGAUCUGAUAAGACAAAUAtt-3' or GAPDH Positive Control siRNA, Cat\# 4390850, Ambion) and a total of $1 \mu \mathrm{g}$ of the GFP-Mis $18 \mathrm{BP} 1^{2-721}$ plasmid and a RFP-H2B plasmid in a 10:1 ratio. Opti-MEM was replaced after $12 \mathrm{~h}$ with fresh growth media. $48 \mathrm{~h}$ after the initial transfection, cells were transfected again with a second round of siRNA at a concentration of $10 \mathrm{nM}$ using RNAiMax in Opti-MEM (Life Technologies). Cells were harvested for western blotting analysis with $3 \mathrm{mM}$ EDTA-PBS or fixed and stained using the protocol above $72 \mathrm{~h}$ after the initial transfection.

Simple siRNA depletions of Mis 18 proteins in cells that stably expressed GFPMis18BP1 were accomplished by plating cells at a density of 10,400 cells $/ \mathrm{cm}^{2}$ in a 6 -well format and letting the cells attach to the plate and coverslips. The following day, cells were treated with $20 \mathrm{nM}$ Silencer ${ }^{\circledR}$ Select siRNA (Mis18BP1, 5'- 
GGAUAUCCAAAUUAUCUCAtt-3'; Mis18 $\alpha$, 5'-GAAGAUGUCUUGAAAGCAUtt3'; Mis18ß 5'-GCACAAUCGCUUAAAAUCAtt-3', Negative Control, Cat\# 4390846, Ambion) using RNAiMax in Opti-MEM. After 12 - 24 h, $1 \mathrm{~mL}$ of full growth media was added to supplement the reduced serum media. Cells were then harvested $48 \mathrm{~h}$ postsiRNA transfection using 3mM EDTA-PBS or fixed and stained using the previously stated protocol.

The degree of siRNA depletion was determined by immunoblot and protein depletion was estimated by a dilution series of control lysates. Cell lysates that corresponded to $1 \times 10^{5}$ cells were separated on $10 \%$ polyacrylamide gels and blotted to nitrocellulose using standard approaches. The nitrocellulose was blocked for $1 \mathrm{~h}$ in blocking buffer: 5\% milk in TBST (137 mM NaCl, $2.7 \mathrm{mM} \mathrm{KCl,} 25 \mathrm{mM}$ Tris Base, $0.1 \%$ Tween 20). Primary antibodies were incubated overnight in blocking buffer at $4^{\circ} \mathrm{C}$ [antiGFP (Custom made by Covance), c-Myc 9E10 (Santa Cruz Biotechnology, sc-40), anti- $\beta$ Tubulin clone AA2, Mis18BP1 (Bethyl Laboratories, Inc., Cat\# A302-825A and Cat\# A302-824A), and Mis18ß (BL10295, Bethyl Laboratories, Inc.). HRP conjugated secondary antibodies were incubated in blocking buffer at $40 \mathrm{ng} / \mathrm{mL}$ for $1 \mathrm{~h}$ at room temperature (Goat-anti-mouse, Cat\# 115-035-003; Goat-anti-rabbit, Cat\# 111-0.35-003, Jackson ImmunoResearch Laboratories, Inc.). HRP was detected using the West Pico ECL Regent (Pierce) chemiluminescent substrate. 


\section{Size exclusion chromatography}

HeLa cells stably expressing GFP-Mis18 $\alpha$ (Iain Cheeseman, MIT) were plated and grown to $80 \%$ confluence. Cells were treated with $0.1 \mu \mathrm{g} / \mathrm{mL}$ nocodazole for $14 \mathrm{~h}$ and mitotic cells were collected by washing the plate with media and collecting detached cells. Cell pellets were brought up in ice-cold lysis buffer (3.75 mM Tris pH 7.5, $20 \mathrm{mM}$ $\mathrm{KCl}, 0.5 \mathrm{mM}$ EDTA, 0.5mM DTT, $0.05 \mathrm{mM}$ spermidine, $0.125 \mathrm{mM}$ spermine, $1 \mathrm{mM}$ PMSF, $200 \mu \mathrm{M}$ sodium vanadate, $50 \mathrm{mM} \beta$-glycerophosphate, $0.1 \%$ digitonin, $1 \mathrm{X}$ Roche Protease Inhibitors). Cells were disrupted on ice using a Wheaton glass dounce with a tight pestle. Lysates were centrifuged at $1000 \mathrm{~g}$ for $5 \mathrm{~min}$ and dialyzed into a second buffer (50mM Tris-HCl pH 7.5, 250mM NaCl, 0.5mM DTT, 2.5\% glycerol, and $0.05 \%$ $\mathrm{NP}-40$ ). Lysates were cleared by centrifugation for $10 \mathrm{~min}$ at $1000 \mathrm{~g}$ and separated by FPLC size exclusion chromatography in the dialysis buffer using a Superdex 200 10/300 column (GE Healthcare). Separation was conducted using a Bio-Rad Duo-flow FPLC and $0.5 \mathrm{ml}$ fractions were collected. Fractions were concentrated using TCA precipitation, prior to immunoblotting.

\section{Immunoprecipitation}

HEK cells were plated at a 1:8 dilution from a confluent plate and allowed to adhere to the culture dish overnight. The following day the cells were cotransfected with GFP-Mis18 $\alpha$ and Myc-tagged Mis18BP1 constructs using calcium phosphate. After $48 \mathrm{~h}$, cells were harvested using $3 \mathrm{mM}$ EDTA-PBS and flash frozen at $-80^{\circ} \mathrm{C}$. Cells were thawed on ice and resuspended in $1 \mathrm{~mL}$ cold RIPA buffer with protease and phosphatase 
inhibitors (50 mM Tris $\mathrm{HCl} \mathrm{pH} 8.0,150 \mathrm{mM} \mathrm{NaCl}, 1 \% \mathrm{NP}-40,0.5 \%$ sodium deoxycholate, $0.1 \%$ SDS, $0.5 \mathrm{mM}$ PMSF, $50 \mathrm{mM} \beta$-glycerophosphate, $200 \mu \mathrm{M}$ sodium vanadate, $5 \mathrm{mM}$ sodium fluoride, $1 \mathrm{X}$ Roche Protease Inhibitors). Lysates were incubated on ice for $15 \mathrm{~min}$ with vortexing, centrifuged for $10 \mathrm{~min}$ at $10000 \mathrm{~g}$ at $4^{\circ} \mathrm{C}$, and cleared for $1 \mathrm{~h}$ with Affi-Prep Protein A Support slurry (Cat\# 156-0006, Bio-Rad). Cleared lysates were then incubated with $1 \mu \mathrm{g}$ anti-Myc 9E10 antibody (Santa Cruz Biotechnology, sc-40) overnight at $4^{\circ} \mathrm{C}$ on a rotator. Fresh protein A support was blocked for $1 \mathrm{~h}$ at $4^{\circ} \mathrm{C}$ with $1 \mathrm{mg} / \mathrm{mL}$ BSA in PBS. Antibody-lysate mixtures were incubated with the blocked protein A support for $5.25 \mathrm{~h}$ at $4^{\circ} \mathrm{C}$. Protein A support was washed 3 times with PBST (PBS, 0.1\% Tween 20, 1X Roche Protease Inhibitors) and bound proteins were eluted by boiling in SDS-PAGE sample buffer (60 mM Tris HCl/SDS pH 6.8, 5\%

glycerol, 1.67\% SDS, 0.01\% bromophenol blue, $1 \% \beta$-mercaptoethanol). Bound fractions and inputs were separated on an $8 \%$ polyacrylamide gel and blotted according to the above protocol using anti-GFP (Custom made by Covance), c-Myc 9E10 (Santa Cruz Biotechnology, sc-40) primary antibodies.

\section{Recombinant proteins and in vitro pull-downs}

Mis18BP1 was cloned into a modified pMal-c2 vector containing an attR Gateway ${ }^{\circledR}$ cloning cassette inserted into the BamH1 cut site after the N-terminal MBP gene. Mis18BP1 harboring a C-terminal 6X-His tag was cloned into the modified pMalc2 vector using LR Clonase II (Cat\# 11791020, Life Technologies). A StrepHA Gateway ${ }^{\circledR}$ vector was made from an existing 6X-his Gateway ${ }^{\circledR}$ vector (Addgene, pDest- 
527) by removing the $6 \mathrm{X}$-His tag with Nde1 and BglII and replacing it with a StrepHA tag digested with the same enzymes. Entry clones of Mis18 $\alpha$ and Mis $18 \beta$ were generated by recombination from PCR products according to the Gateway® cloning protocol (Life Technologies) and were recombined into the StrepHA acceptor vector with LR Clonase II. The CENP-C fragment (a.a. 694-943) was recombined into a HisNusA Gateway® vector (Addgene, pDest-544) using LR clonase II.

Recombinant MBP-tagged Mis18BP1, StrepHA-Mis18 $\alpha$, StrepHA-Mis18 $\beta$, and His-NusA-CENP-C (a.a. 694-943) proteins were expressed in the Rosetta ${ }^{\mathrm{TM}}$ (DE3) pLysS bacterial strain. Transformed bacteria were grown in LB media to an OD of 0.6 at $37^{\circ} \mathrm{C}$, cooled to $18^{\circ} \mathrm{C}$, and protein expression was induced with $0.1 \mathrm{mM}$ IPTG for $16 \mathrm{~h}$ at $18^{\circ} \mathrm{C}$. Pellets were lysed in $50 \mathrm{mM}$ Tris- $\mathrm{HCl}, 350 \mathrm{mM} \mathrm{NaCl}, 0.5 \mathrm{mM} \mathrm{CaCl}_{2}, 10 \%$ glycerol, 0.1\% NP-40, $5 \mathrm{mM} \beta$-mercaptoethanol, LPC, and 1mM PMSF using a steel Wheaton dounce homogenizer. For His-tagged proteins, $20 \mathrm{mM}$ imidazole was added to the lysis buffer and $40 \mathrm{mM}$ imidazole was added to the wash buffer. Strep-tagged proteins were purified with Strep-Tactin Superflow Plus (Qiagen) and eluted in a buffer containing 2.5 $\mathrm{mM}$ d-desthiobiotin. His-tagged proteins were purified using Ni-NTA agarose (Qiagen) eluted with $250 \mathrm{mM}$ imidazole. Mis18BP1 was initially purified using its C-terminal Histag using the protocol above. It was further purified using a Superdex 200 10/300 column (GE-Healthcare). Briefly, elutions were pooled and run over the column in the above buffer (without LPC and PMSF). Peak fractions were pooled and concentrated on NiNTA agarose and eluted in the same buffer containing $250 \mathrm{mM}$ imidazole. 
In-vitro pull-downs were performed in buffer containing $50 \mathrm{mM}$ Tris- $\mathrm{HCl} \mathrm{pH}$ 7.5, $250 \mathrm{mM} \mathrm{NaCl}, 20 \mathrm{mM} \mathrm{MgCl} 2,0.5 \%$ NP-40, 10\% glycerol, and $5 \mathrm{mM} \beta$ mercaptoethanol. Recombinant proteins combined at 1:1 molar ratio and incubated for 3 $\mathrm{h}$ at room temperature. Affinity matrices were pre-incubated for $1 \mathrm{~h}$ at room temperature in the same buffer supplemented with $0.2 \mathrm{mg} / \mathrm{mL}$ BSA. The blocked matrices were added to the pre-formed complexes and incubated for $40 \mathrm{~min}$ at room temperature. Wash buffer for Ni-NTA pull-downs, which was supplemented with $40 \mathrm{mM}$ imidazole. The matrices were resuspended in sample buffer boiled. Immunoblots were performed using antibodies against the 6X-His tags (sc-803, Santa Cruz Biotechnologies), MBP tag (New England Biolabs), Mis18ß (BL10295, Bethyl Laboratories, Inc.), the HA.11 epitope tag (Covance), and NusA tag (Novus Biologicals).

\section{Nocodazole release and inhibitor treatments}

HeLa cells either were left to randomly cycle, or were treated with $100 \mathrm{ng} / \mathrm{mL}$ of nocodazole in DMSO for $12-14 \mathrm{~h}$. Cells that were to be released were harvested by mitotic shake off, and pelleted at $1000 \mathrm{~g}$ for 3 min. The cells were washed $5 \mathrm{X}$ with warm media, pelleting after each wash. Then the released cells were plated again and incubated at $37^{\circ} \mathrm{C}$ for $3 \mathrm{~h}$. Cells were harvested with $3 \mathrm{mM}$ EDTA-PBS, counted and pelleted. Cell pellets were lysed in $1 \mathrm{X}$ sample buffer, pulled through an insulin syringe and boiled for 5 min prior to analyzing by western blot using the protocol described above.

U2OS-LacO were treated for $1 \mathrm{~h}$ with $25 \mu \mathrm{M}$ purvalanol A, $1 \mu \mathrm{M}$ BI 2536 or DMSO as a vehicle control. Since the inhibitors had differing effective concentrations, 
the volume of DMSO was matched to the purvalanol A treatment, since the volume of addition was greater. After $1 \mathrm{~h}$ at $37^{\circ} \mathrm{C}$, cells were fixed and stained according to the above protocol and analyzed by immunofluorescence microscopy. 


\section{Acknowledgements}

We thank Iain Cheeseman for the GFP-Mis18 $\alpha$ cell line and Don Cleveland for the CENP-T antibody. We thank Dan Burke and Todd Stukenberg for helpful comments on the manuscript. D.R.F was supported by a Research Scholar Award from the American Cancer Society and a Basil O’Conner Award from the March of Dimes. M.E.S. and I.K.N. were supported by NCI training grant 5T32CA00910937. C.M.K. was supported by a Harrison Award from the University of Virginia. 


\section{Supplemental Data}

\section{Supplemental Figure S3-1. Endogenous Mis18BP1 is not required for Mis18BP1 ${ }^{2-721}$ to localize to centromeres.}

(A) Anti-GFP immunoblot showing the transient expression of the GFP-Mis18BP1 constructs expressed in U2OS cells for the immunofluorescence subcellular localization analysis in Figure 3-1. (B) Diagram of the transfection protocol for the simultaneous siRNA depletion of endogenous Mis18BP1 and the expression of exogenous GFPMis $18 \mathrm{BP} 1^{2-721}$ in U2OS cells. (C) Immunoblot of whole cell extracts from GAPDH or Mis18BP1 siRNA treated U2OS cells also expressing GFP-Mis18BP1 $1^{2-721}$. AntiMis18BP1 blot shows protein level of endogenous Mis18BP1 alone. Anti-tubulin is used as a loading control. Anti-GFP western blot shows exogenous GFP-Mis 18BP1 $1^{2-721}$ expression. (D) Representative images of U2OS cells treated as diagramed in B. Centromeres are indicated by staining with polyclonal anti-CENP-T antibodies. Scale bar represents $5 \mu \mathrm{m}$. (E) Graph showing the percentage of randomly cycling, transfected cells with GFP signal localized to CENP-T foci. Error bars indicate the standard deviation between replicates. 


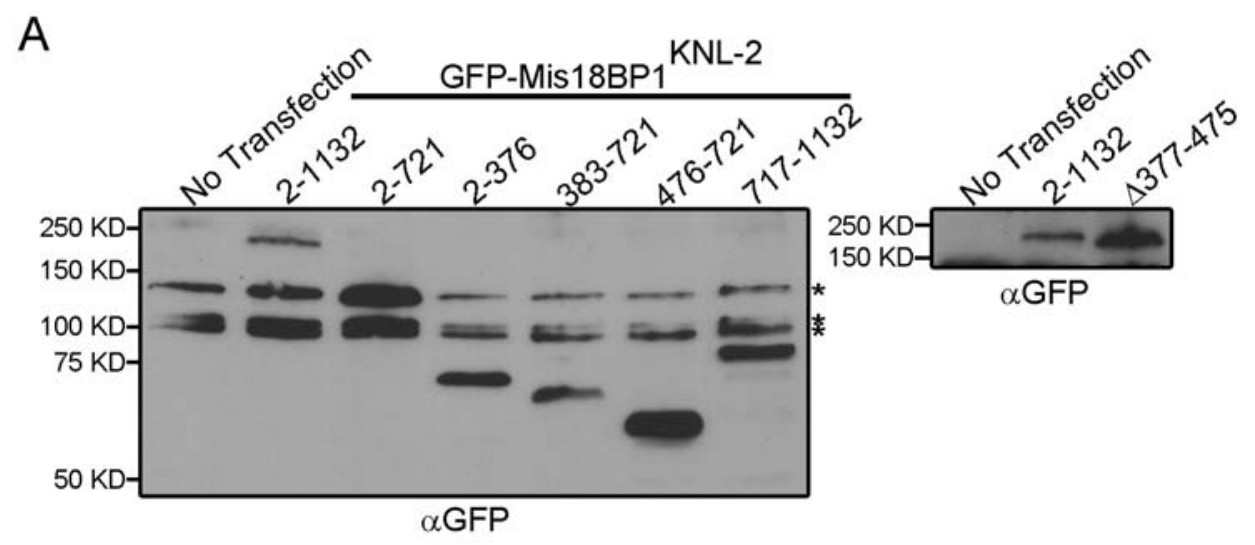

B
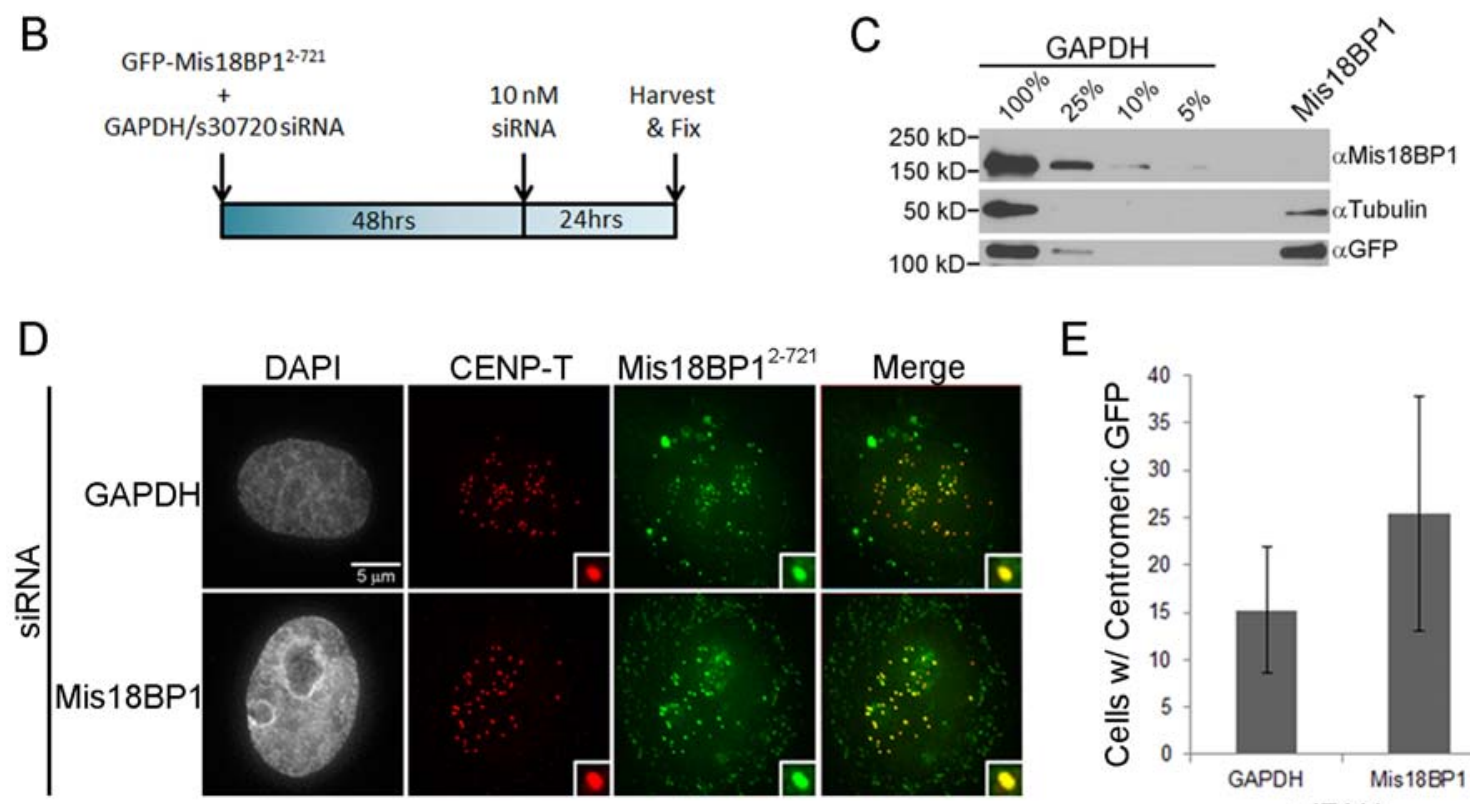

E

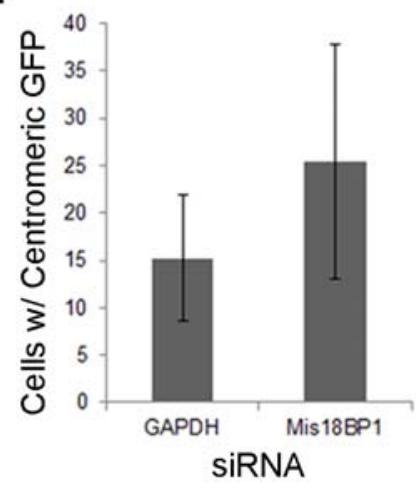

Figure S3-1 


\section{Supplemental Figure S3-2. Decrease in CENP-A levels validates Mis18 complex} depletion.

(A) Representative images of HeLa T-Rex cells that stably express GFP-Mis18BP1, which were treated with negative control siRNA or siRNA against Mis $18 \alpha$, Mis18 $\beta$ or Mis18BP1. Cells were stained with polyclonal antibodies against CENP-T to mark centromere location. CENP-A protein levels at centromeres were assayed by staining with an antibody against endogenous CENP-A. Scale bar represents $5 \mu \mathrm{m}$. (B) Bar graph depicts the background corrected, CENP-A integrated intensity at centromeres. Error bars indicate the standard deviation across three replicates. Double asterisks represent a pvalue of $<0.01$ with respect to the negative siRNA control, as calculated by the Student's t-test.

A

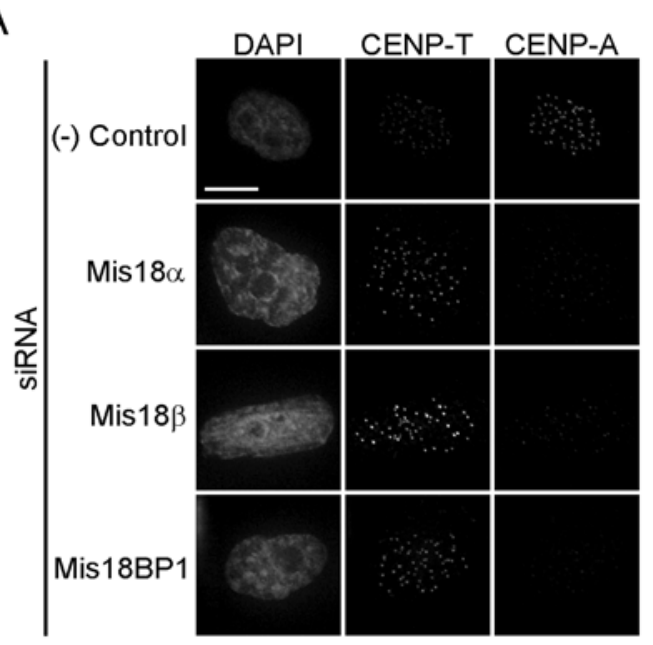

B

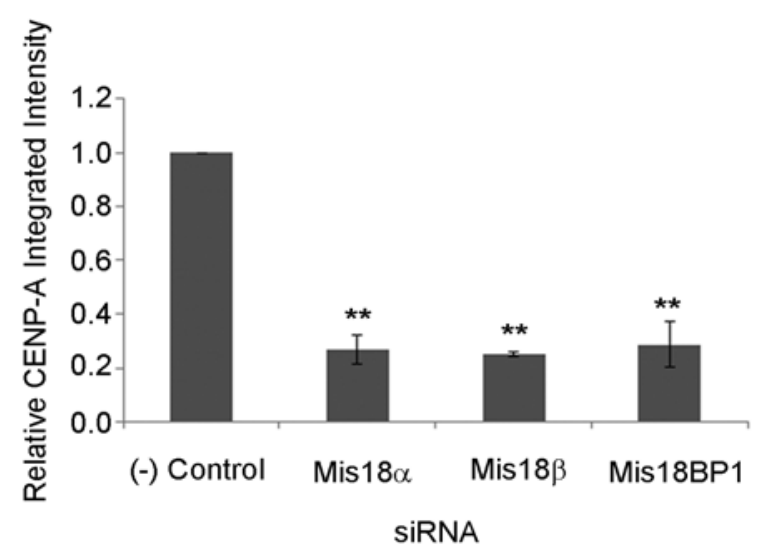

Figure S3-2 


\section{Supplemental Figure S3-3. Full recruitment of the Mis18 complex by CENP-C}

\section{requires multiple interactions}

(A) Representative images of mCherry-LacI-CENP-C ${ }^{694-943}$ recruiting GFP-Mis18BP1 constructs to the LacO array. Centromeres are marked by an antibody against CENP-A. Insets highlight the array. Scale bar represents $5 \mu \mathrm{m}$. (B) Graph indicates the recruitment of GFP-Mis18BP1 fragments to the array, over the average nuclear GFP signal, when mCherry-LacI-CENP-C $\mathrm{C}^{694-943}$ was used as bait. Error bars describe the standard deviation across replicates. Asterisk indicates a p-value of $<0.05$ with respect to the recruitment of full-length GFP-Mis18BP1, as calculated by the Kruskal-Wallis test. 


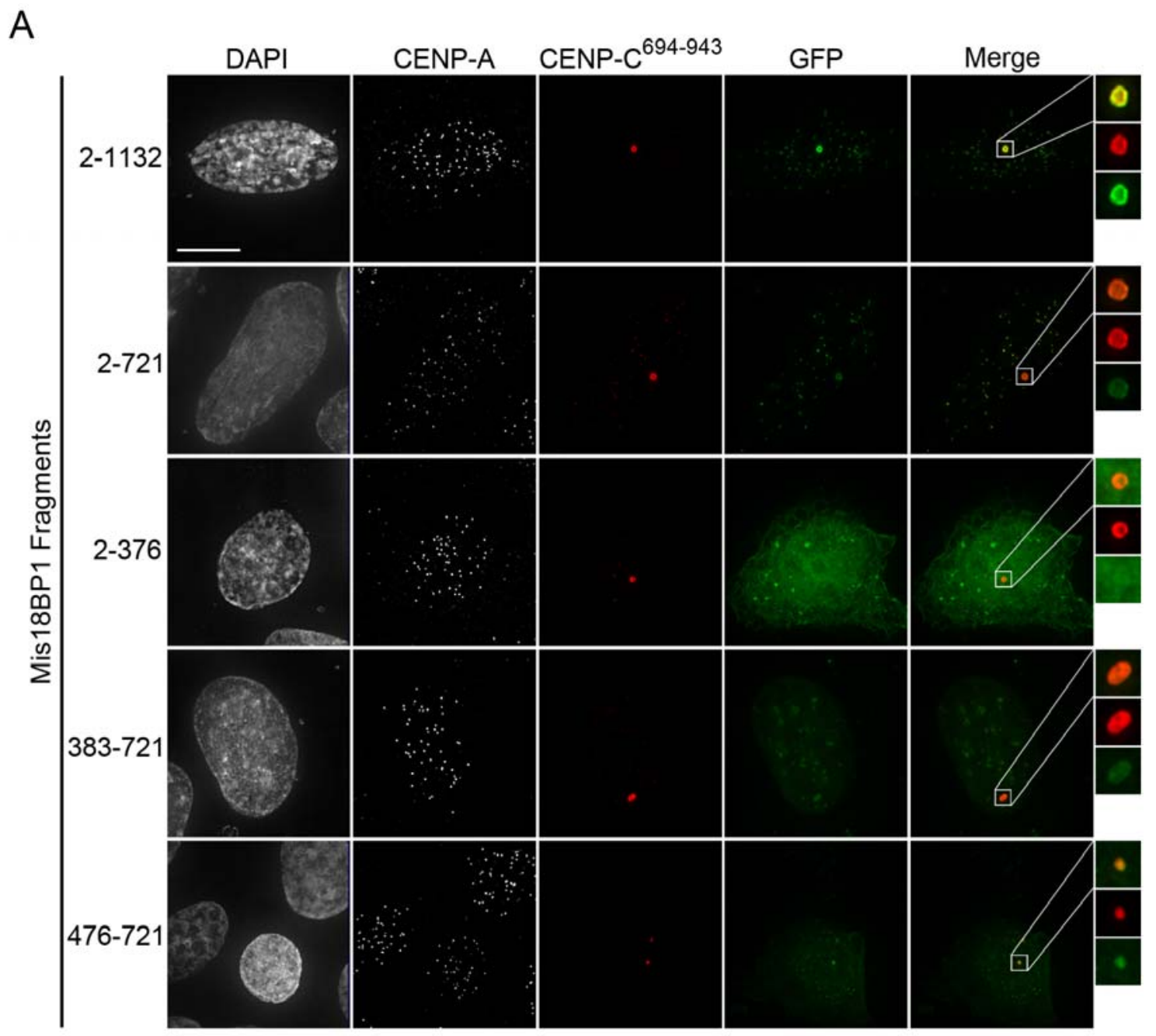

B

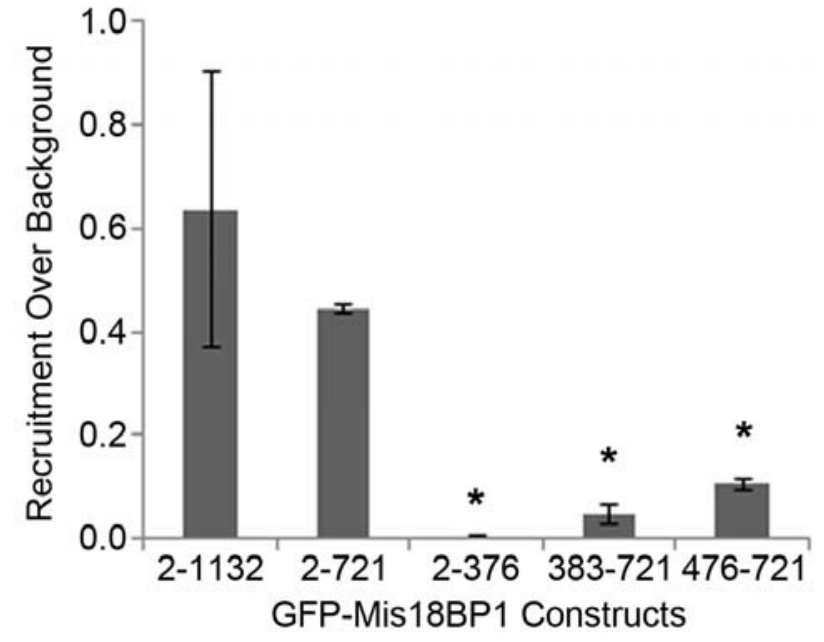

Figure S3-3 


\section{Chapter 4 - Preliminary Data and Future Directions}




\begin{abstract}
In this final chapter, I will focus on preliminary results from three additional projects and will propose several avenues for future research. The first section will highlight a possible new role for Mis18BP1 in centromeric stability and structure. After initiating HJURP-mediated CENP-A deposition upon mitotic exit, Mis18BP1 localization shifts to pericentric heterochromatin in late G1 and specifically binds HP1 $\alpha$. In the second section, I will describe recently acquired data that suggests additional mechanisms by which the Mis 18 complex is recruited to existing centromeric chromatin. In addition to the known interactions between the Mis18 proteins and CENP-C, there appears to be an additional dependency on the CENP-H/I/K complex for proper Mis 18 complex localization in vivo. In the final section, I will describe data that show that the conserved SANT domain of Mis18BP1 does not bind unmodified histone tails. The results indicate that further experimentation is required to understand the role of this highly conserved domain in Mis18 complex function and to determine the chromatin contacts that underlie the centromeric binding of the Mis 18 complex.
\end{abstract}




\section{Mis18BP1-HP1 $\alpha$ interaction at pericentric heterochromatin}

\section{Introduction:}

A conserved feature of centromere architecture is the presence of heterochromatic regions that flank the CENP-A containing domain of each chromosome. Pericentric heterochromatin is constitutively present throughout the cell cycle and is maintained from generation to generation $[176,177]$. The presence of constitutive pericentric heterochromatin is essential for proper centromere function and genomic fidelity during chromosome segregation [178-180]. In fact, in immunodeficiency-centromeric instability-facial anomalies syndrome 1 (ICF1) defects of heterochromatin, specifically at the pericentromere, result in significant chromosomal aberrations. Patient samples display highly irregular karyotypes that contain broken chromosomes and fused fragments, which are indicative of repeated chromosome segregation errors [181].

In flies and fission yeast, de novo CENP-A deposition during neocentromere formation prefers to occur near constitutive heterochromatin domains, such as at the border of pericentric heterochromatin [16, 60]. Additionally, ectopic targeting of the heterochromatin protein, HP1, to a discrete locus generates an artificial hotspot for noncentromeric incorporation of over-expressed CID (CENP-A) in drosophila [60]. In addition to facilitating a permissive environment for nearby CENP-A recruitment, pericentric heterochromatin is hypothesized to lend structure and stability to the centromeric region of chromosomes during mitosis and to facilitate proper kinetochore attachment to the mitotic spindle [182]. Pericentric heterochromatin also ensures 
chromosome stability by recruiting cohesin, which facilitates sister-sister chromatid binding and biorientation during mitosis [179, 183].

Pericentric heterochromatin is identified by a specific epigenetic landscape, including the presence of $\mathrm{H} 3 \mathrm{~K} 9$ trimethylation as well as hypoacetylation of histone tails $[184,185]$. Effector proteins recognize these marks and bind to this region in order to mediate pericentric heterochromatin identity and function. Swi6 was originally discovered in fission yeast, and it was determined to be a quintessential non-histone, heterochromatin protein in drosophila [186, 187]. The human homolog, of Swi6 is heterochromatin protein 1 (HP1) [188]. Humans encode three different protein homologs of Swi6: HP1 $\alpha$, HP1 $\beta$ and HP1 $\gamma$. HP1 $\alpha$ and HP1 $\beta$ contain N-terminal chromo domains and C-terminal chromo shadow domains, and preferentially localize to centromeric and pericentric regions of chromosomes [189].

My work discussed in previous chapters, as well as that of others, has delineated a clear role for the Mis18 complex in the direct recruitment of HJURP to the centromere for CENP-A deposition $[18,151]$. However, only the N-terminal 376 amino acids of Mis18BP1 are required to interact with Mis18 $\alpha$ and Mis18 $\beta$, who alone direct HJURP recruitment through their C-terminal coiled-coil domains [90, 151]. Therefore, other aspects of Mis18BP1 may mediate additional, CENP-A deposition independent functions of the Mis18BP1.

In fission yeast, the Mis18 protein has been implicated in maintaining the epigenetic environment of the centromere. At the restrictive temperature, Mis 18 temperature sensitive strains have increased acetylation of histone $\mathrm{H} 4$ in centromeric 
nucleosomes [91]. This indicates that the presence of the Mis18 protein helps to maintains H4 in a hypoactelyated state. Studies on human artificial chromosomes (HACs) have also discovered a link between $\mathrm{H} 3 \mathrm{~K} 9$ acetylation status and the requirement of the Mis 18 complex for de novo CENP-A deposition [47]. Tethering histone acetyltransferase (HAT) activity to the alphaloid repeats of a HAC can greatly increase the efficiency of generating a functional centromere. Interestingly, tethered HAT activity at the HAC bypasses the requirement for human Mis $18 \alpha$, which strongly suggests that the Mis 18 complex may assist in generating the particular epigenetic signature for CENP-A incorporation. Mis18BP1 also contains two highly conserved domains, the SANT domain and the SANT-associated (SANTA) domain [92]. SANT domains are common structure motifs found in many chromatin modification complexes, and the SANTA domain may mediate protein-protein interactions through conserved hydrophobic residues $[111,112]$.

Throughout my localization studies of the Mis 18 complex, I noticed two consistent patterns of Mis18BP1 localization. Cells with early G1 morphology tended to display highly focal recruitment of Mis18BP1, which tightly overlapped with CENP-A staining. The second population consisted of interphase cells in which Mis18BP1 localized to broader and more diffuse patches. These patches associated with clusters of centromeres, which tended to form around the edges of nucleoli. I defined this second pattern as diffuse centromeric localization. This diffuse pattern of localization was reminiscent of HP1 $\alpha$ staining of pericentric heterochromatin [190]. Therefore, I decided to ascertain a possible linkage between the Mis18 complex and the proteins that mediate heterochromatin structure and function at the pericentromere. 


\section{Preliminary results:}

Mis18BP1 accumulates at pericentric heterochromatin in late G1

I generated a HeLa Flp-In T-Rex cell line that stably expressed full-length GFPMis18BP1 by recombination. The GFP-tagged protein had focal localization in early G1 that completely colocalized with CENP-A foci, which was expected. As described above, a population of interphase nuclei had a slightly different localization pattern. Colocalizing with clusters of centromeres were larger and more diffuse patches of GFP-Mis18BP1 signal, which I declared diffuse centromeric localization. It had previously been discovered that the Mis18 complex can remain associated with the centromere far into G1, and the cells that displayed the diffuse centromeric pattern of GFP-Mis18BP1 localization were clearly non-mitotics [133]. Therefore, I hypothesized that the pattern of diffuse centromeric localization of Mis18BP1 follows the protein's initial focal localization that occurs directly after mitotic exit.

In order to ascertain whether the diffuse localization pattern indeed occurred after the focal localization seen in early G1 cells, I blocked HeLa GFP-Mis18BP1 expressing cells for 12 hours in nocodazole. The mitotic population was collected by mitotic shake off and the cells were washed and replated onto glass coverslips. At several time points post-release, the cells were fixed and stained for nuclei with DAPI. The fixed cells were analyzed by immunofluorescence microscopy for mitotic figures as well as for the GFPMis18BP1 localization pattern (Figure 4-1, A - C).

After one hour of mitotic release, a small portion of cells had exited mitosis, which correlated with less than $10 \%$ of cells with GFP-Mis $18 \mathrm{BP} 1$ localized to 
centromeres in tight foci (Figure 4-1, A - C). As more cells exited mitosis, the proportion of cells that had the focal GFP-Mis18BP1 localization pattern increased. Interestingly at five hours after nocodazole washout the focal centromeric GFP-Mis18BP1 population began to decline and a new population of cells emerged with the diffuse centromeric localization pattern. Over the next several hours, the focal localization pattern indicative of early G1 continued to decrease and was replaced by a majority of cells portraying the centromere diffuse phenotype.

As described earlier in chapter 3, I had generated a series of GFP-tagged Mis18BP1 fragments in order to address which portions of Mis18BP1 were required for centromeric localization (Figure 4-1D). Using these constructs, I assayed which of these GFP-Mis18BP1 fragments were sufficient to display this centromere diffuse phenotype. As expected, transient transfection of full-length GFP-Mis18BP1 had a population of cells that displayed a diffuse centromeric pattern similar to what was seen in the stable cell lines (Figure 4-1E). The GFP-Mis18BP1 $1^{2-721}$ fragment, which was sufficient for full centromere localization, also had a population of cells that displayed this diffuse centromeric localization pattern. Finally, GFP-Mis18BP $1^{383-721}$ was also able to localize into a diffuse pattern around centromeric clusters, despite the fact that this fragment never localized to early G1 foci. Mis $18 \mathrm{BP} 1^{383-721}$ contains the conserved SANTA domain, but not the N-terminal Mis $18 \alpha$ binding domain. Therefore, this localization pattern may be dependent on the presence of the SANTA domain of Mis18BP1.

This diffuse pattern of GFP staining was reminiscent of HP1 $\alpha$ staining of pericentric heterochromatin in interphase [190]. Therefore, I attempted to determine if 
GFP-Mis 18BP1 foci colocalized with HP1 $\alpha$ staining. Using the HP1 $\alpha$ antibody I was able to procure, I saw little colocalization between Mis18BP1 and HP1 $\alpha$ above background. In Figure 4-1F, I highlighted one of the better examples of HP1 $\alpha$ staining. In this image, there were several HP1 $\alpha$ foci that colocalized with diffuse GFP-Mis18BP1 patches (Figure 4-1F, white arrows). These HP1 $\alpha$ foci tended to corresponded to centromeres that had clustered with neighboring centromeres, as indicated by the staining against endogenous CENP-A. This may indicate that this putative Mis18BP1heterochromatin interaction prefers the close proximity of other pericentric domains for optimal binding. 
Figure 4-1. Mis18BP1 accumulates at pericentric heterochromatin in late G1.

(A) Representative images of GFP-Mis18BP1 expressing HeLa cells after nocodazole release. Hours post-release indicated in white in the top right. Insets highlight GFP signal localization pattern in greyscale. (B) Graph of the percentage of cells in mitosis at each time point. $\mathrm{N}=1$. (C) Graph depicting the change in GFP localization pattern over time, after nocodazole release. Dark grey bars show the percentage of cells with focal centromeric GFP signal (as in hour 2 in A). Light grey bars show the percentage of cells with diffuse centromeric GFP signal (as in hour 5 in A). $N=1$. (D) Schematic of GFPtagged Mis18BP1 fragments analyzed in E. (E) Graph of the average percentage of transfected cells with diffuse centromeric GFP-Mis18BP1 staining for each fragment. Error bars show the standard deviation across replicates. $N=3$. (F) Image displaying overlap in cells transiently transfected with GFP-Mis 18BP1. GFP signal (green) and staining against endogenous HP1 $\alpha$ (red). Centromeres are indicated with anti-CENP-A staining (white). White arrows indicate colocalization events between Mis18BP1 and HP1 $\alpha$, one of which is highlighted by the boxed enlarged region. Scale bars $=5 \mu \mathrm{m}$. 


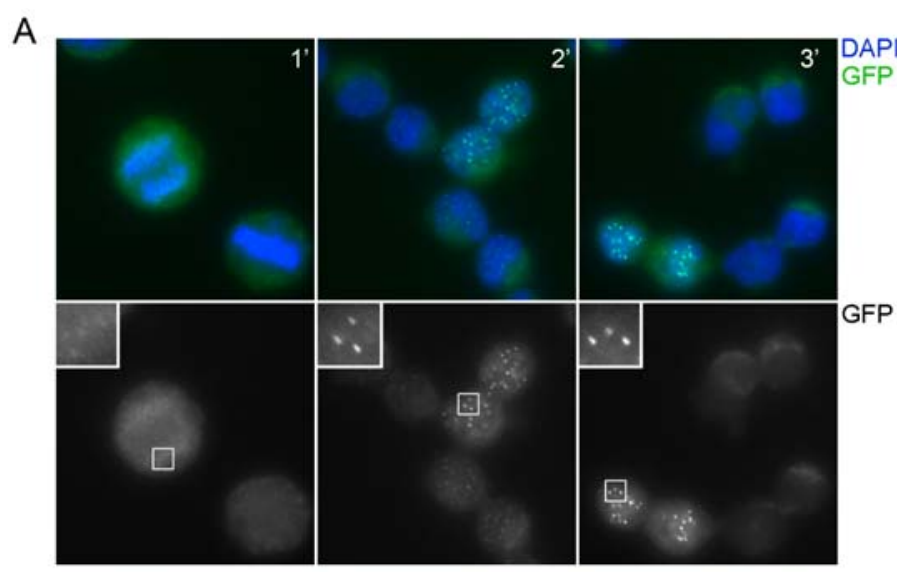

B

Mitotic Index
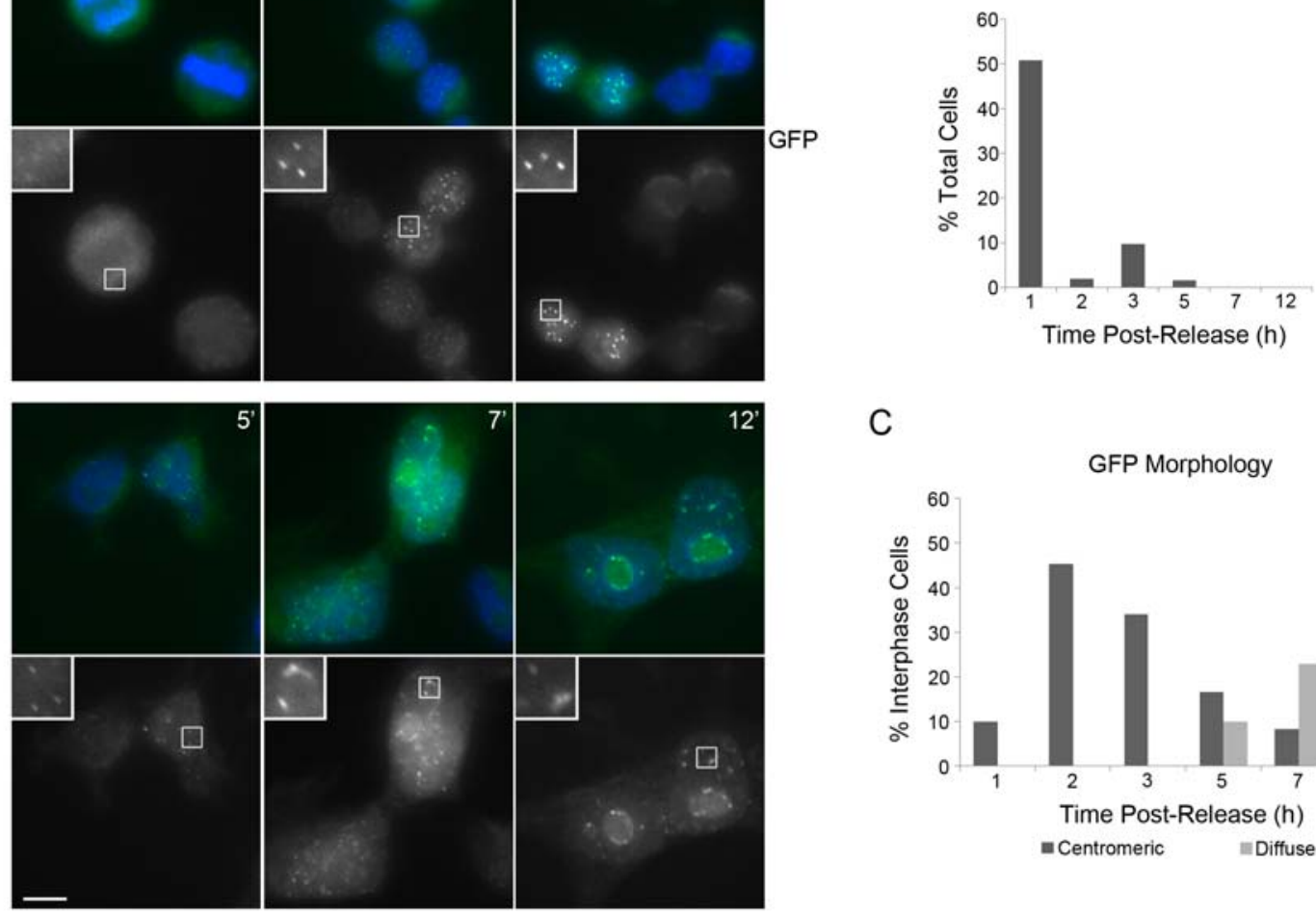

C

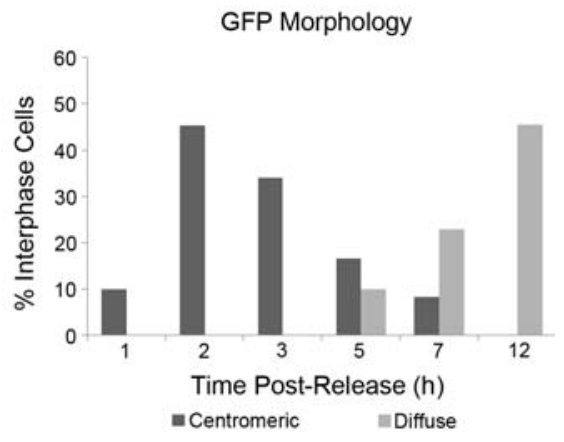

D

E
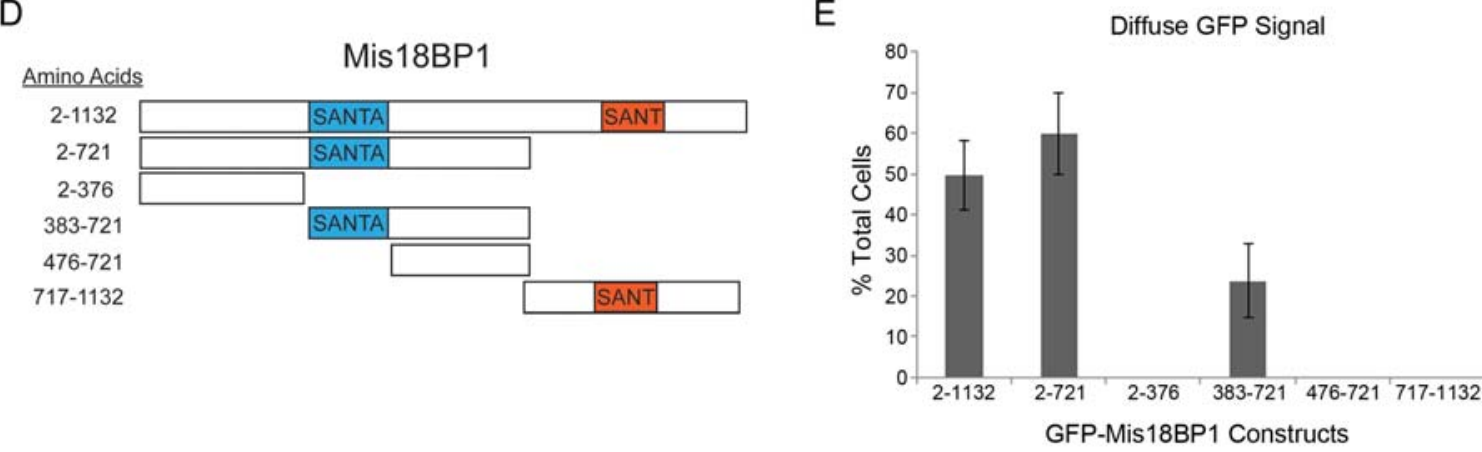

F

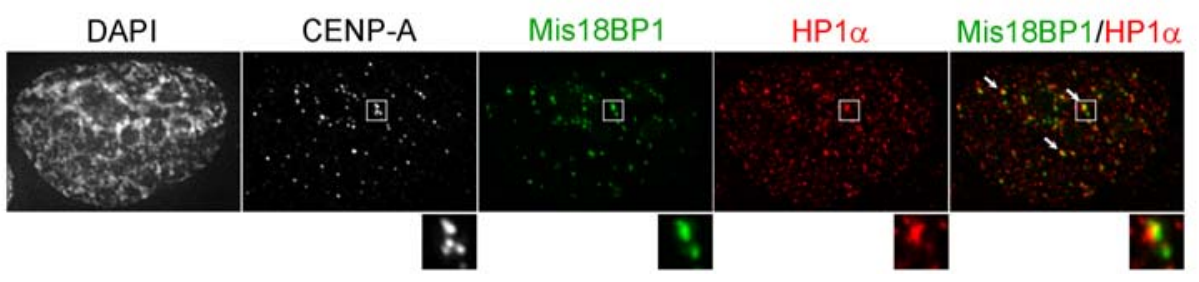

Figure 4-1 


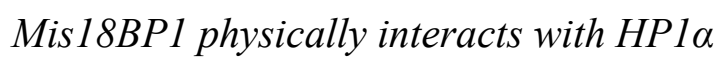

As the limitations of immunofluorescence staining were clear, I performed immunoprecipitations from a cell line that stably expressed GFP-HP1 $\alpha$ in order to confirm the possible relationship between Mis18BP1 and HP1 $\alpha$ (gift from L. Liu). The Mis18 complex does not localize to chromatin in mitosis [90, 91, 164]. Therefore, immunoprecipitations were performed from randomly cycling cells and a mitotic population for comparison. I hypothesized that I would see a more robust Mis18BP1HP1 $\alpha$ interaction in the randomly cycling population, and I would see little or no interaction in mitotically arrested lysates.

Immunoprecipitations were performed first by using an anti-Mis18BP1 antibody and blotting back with antibodies against the GFP-tag of GFP-HP1 $\alpha$ as well as against endogenous HP1 $\alpha$. Contrary to the original hypothesis, Mis18BP1 immunoprecipitations from mitotically arrested cells showed a distinct interaction between Mis18BP1 and both exogenous and endogenous HP1 $\alpha$ (Figure 4-2A, last lane). In a reciprocal experiment, GFP-HP1 $\alpha$ was immunoprecipitated by anti-GFP antibodies from randomly cycling and mitotically arrested lysates. There was an enrichment of Mis18BP1 in the anti-GFP (HP1 $\alpha$ ) immunoprecipitates from nocodazole treated cells. Once again, this strongly implies that there is a definite interaction between Mis18BP1 and HP1 $\alpha$ in human cells, and that some aspect of the mitotic extract facilitates this interaction.

For further confirmation that Mis18BP1 and HP1 $\alpha$ bind, we performed size exclusion chromatography from mitotically arrested cells that stably express GFPMis18 $\alpha$. I generated chromatin-free, mitotic extracts and I.K. Nardi ran the samples over 
a Superdex 200 column, blotting the fractions for Mis 18 complex proteins as well as endogenous HP1 $\alpha$ (Figure 4-2C). HP1 $\alpha$ most clearly co-eluted in a high molecular weight complex with Mis18BP1 (lanes 10 - 12). In addition, a population of HP1 $\alpha$ also cofractionated with the mitotic Mis18 $\alpha$-Mis18 $\beta$ complex previously described in chapter 3 (lanes 15 - 19). HP1 $\alpha$ also eluted alone in another lower molecular weight complex that did not contain proteins of the Mis 18 complex (lanes $21-23$ ). These data indicate that Mis18BP1 and HP1 $\alpha$ interact in a distinct complex in mitotic extracts.

However, immunoprecipitations and size exclusion chromatography do not rule out the possibility that the interaction between Mis18BP1 and HP1 $\alpha$ was mediated by additional factors that bind to each protein. HP1 $\alpha$ and HP1 $\beta$ are both found to localize to pericentromeric heterochromatin [189]. Therefore, I.K. Nardi isolated recombinant fulllength MBP-Mis18BP1 as well as His-tagged HP1 $\alpha$ and HP1 $\beta$ and assessed their interaction in vitro. This allowed us to determine if the Mis18BP1-HP1 $\alpha$ interaction is direct and if there is a specificity for Mis18BP1 to bind HP1 $\alpha$ over another pericentromeric heterochromatin protein.

MBP-Mis18BP1 was incubated with either His-tagged HP1 $\alpha$ or HP1 $\beta$ and possible interactions with Mis18BP1 were isolated on amylose beads. MBP-Mis18BP1 clearly interacted with His-HP1 $\alpha$ in this in vitro pull down assay, but no interaction was seen between MBP-Mis18BP1 and His-HP1 $\beta$ suggesting specificity for HP1 $\alpha$. However, the fraction of His-HP1 $\alpha$ that was isolated on the amylose resin was a small fraction of the total input protein. This may indicate that other binding factors are involved in vivo to 
help stabilize the interaction, or it may indicate that a robust Mis18BP1-HP1 $\alpha$ interaction requires post-translational modifications, not present in the recombinant preparations. 


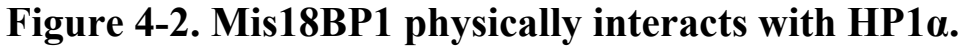

(A) Anti-Mis18BP1 immunoprecipitations from randomly cycling (nocodazole -) and mitotic (nocodazole + ) populations of GFP-HP1 $\alpha$ expressing cells. Rabbit IgG was used as an antibody control. Inputs equal 7\% of total input fraction. (B) Anti-GFP IP from RIPA extracts of GFP-HP1 $\alpha$ expressing cells allowed to randomly cycle (nocodazole -) or else mitotically arrested (nocodazole + ), which was blotted for endogenous Mis18BP1. (C) Mitotic extracts from cells stably expressing GFP-Mis18 $\alpha$ were run over a Superdex 200 size exclusion column. Fractions were blotted for the presence of Mis18BP1, GFPMis $18 \alpha$, Mis $18 \beta$ and HP1 $\alpha$. Arrows highlight the migration of protein standards with the indicated Stokes radii. (D) Amylose resin pull downs of recombinant full-length MBPMis18BP1 incubated with either purified His-tagged HP1 $\alpha$ or HP1 $\beta$ full-length proteins. 
A

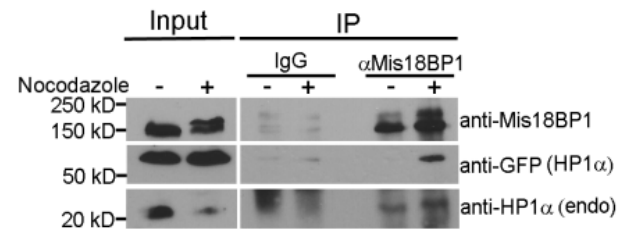

B

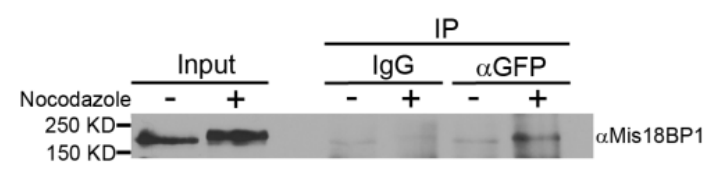

C

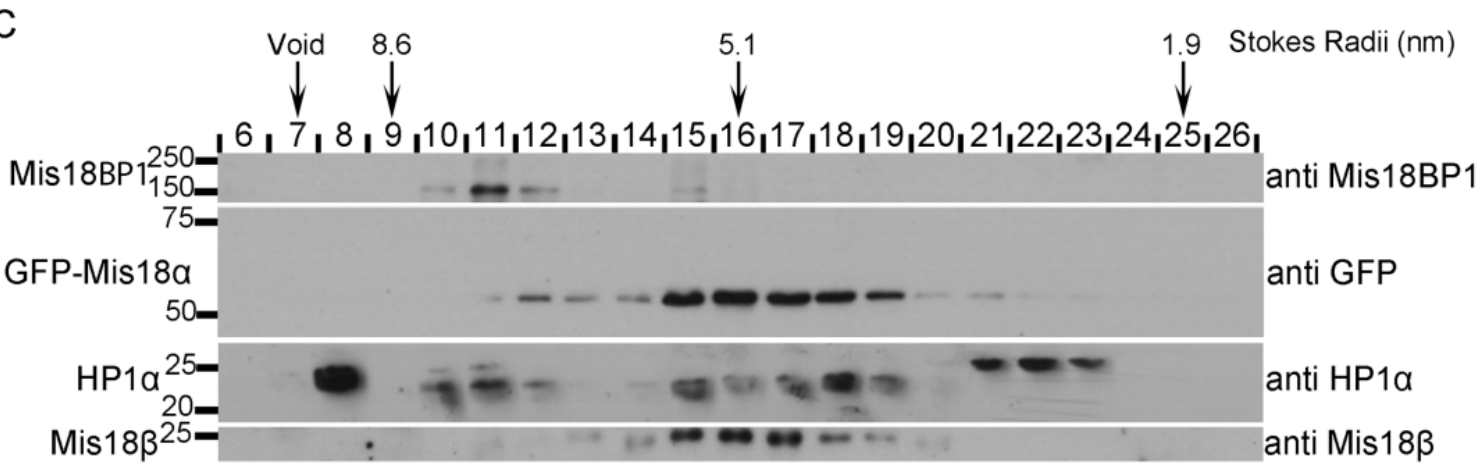

D

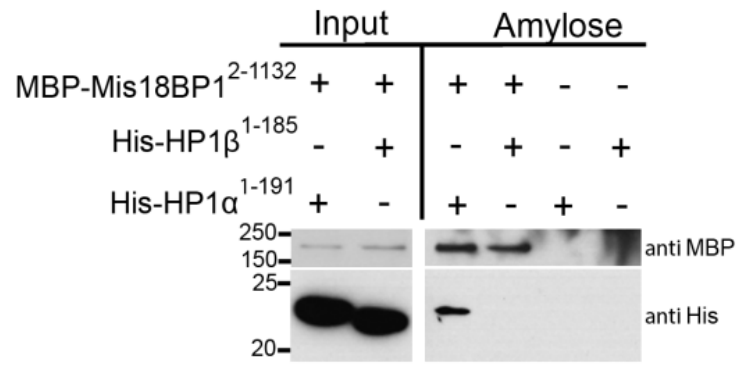

Figure 4-2

SEC and amylose pull downs were performed by I.K. Nardi 
HP1 1 is not required for centromeric recruitment of the Mis 18 complex

Previous studies have shown that HP1 can generate a hotspot of CENP-A deposition outside of endogenous centromere loci [60]. Therefore, I tested whether HP1 $\alpha$ was required for the centromeric localization of the Mis 18 complex in early G1. I performed siRNA depletions of HP1 $\alpha$ from cells stably expressing GFP-Mis18BP1. Cells were treated with HP1 $\alpha$ siRNA over the course of 72 hours, using the protocol shown in Figure 4-3A. After siRNA treatment, GFP-Mis18BP1 cells were analyzed by western blot and immunofluorescence. Western blots against HP1 $\alpha$ showed that HP1 $\alpha$ protein levels were below $10 \%$ of untreated lysates in cells that had been treated with siRNA against HP1 $\alpha$ (Figure 4-3B). Anti-Mis18BP1 blots indicated that HP1 $\alpha$ depletion did not affect protein levels of endogenous Mis18BP1 or the GFP-tagged protein, as indicated by the doublet band. Immunofluorescence analysis showed that depleting HP1 $\alpha$ did not affect the proportion of randomly cycling cells that displayed focal GFP-Mis18BP1 localization to centromeres in G1. Additionally, the CENP-A levels at the centromere did not decrease appreciably in the HP1 $\alpha$ depletions as compared to untreated controls.

A similar experiment was repeated in a cell line that stably expressed GFPMis18 $\alpha$. Depletion of endogenous HP1 $\alpha$ did not affect the centromeric localization of Mis18 $\alpha$, similar what was seen with GFP-Mis18BP1. These results indicated that while Mis18BP1 and HP1 $\alpha$ do interact in vivo and in vitro, this interaction may mediate additional functions of the Mis18 complex outside of its role in the CENP-A deposition pathway. 
Figure 4-3. HP1 $\alpha$ is not required for centromeric recruitment of the Mis18 complex. (A) Diagram of the siRNA treatment protocol used throughout the figure. (B) Western blots of whole cell lysates from HP1 $\alpha$ depleted GFP-Mis18BP1 expressing HeLa T-Rex cells. Standard curve was generated from untreated extracts. (C) Representative images comparing GFP-Mis18BP1 localization in untreated and HP1 $\alpha$ depleted cells. AntiCENP-T staining was used as a marker of endogenous centromeres. Scale bars $=5 \mu \mathrm{m}$. (D) Graph of the average percentage of randomly cycling cells that have GFP-Mis18BP1 signal at centromeres. Error bars indicate the standard deviation across replicates. $\mathrm{N}=2$. (E) Representative images comparing GFP-Mis18 $\alpha$ localization in GAPDH control siRNA and HP1 $\alpha$ siRNA treated cells that stably express GFP-Mis $18 \alpha$. Anti-CENP-T staining was used as a marker of endogenous centromeres. Scale bars $=5 \mu \mathrm{m}$. (F) Graph of the percentage of randomly cycling cells that have GFP-Mis18 $\alpha$ signal at centromeres. $\mathrm{N}=1$. 
A

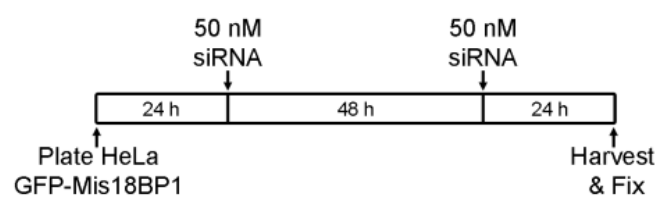

B

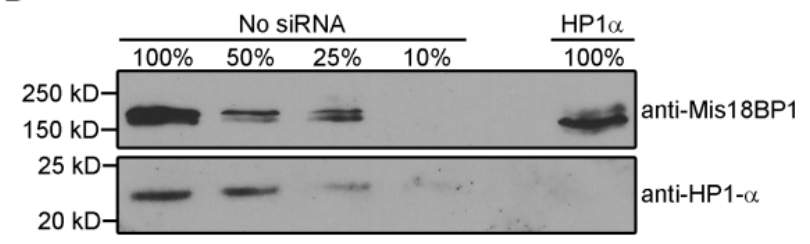

C

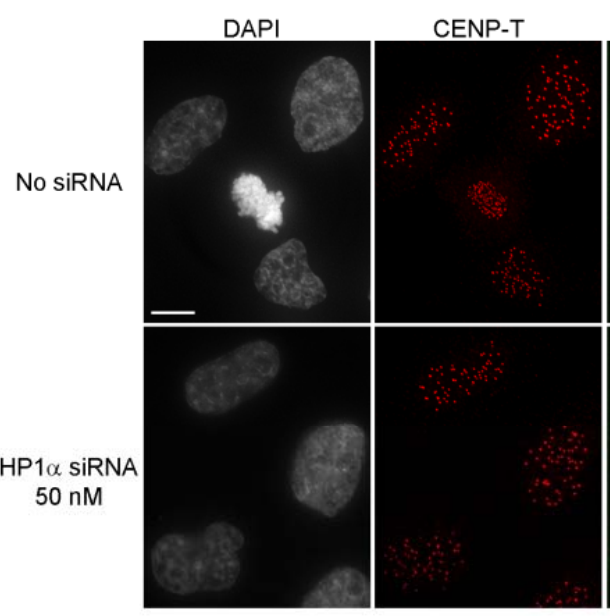

Mis18BP1

Mis18BP1/

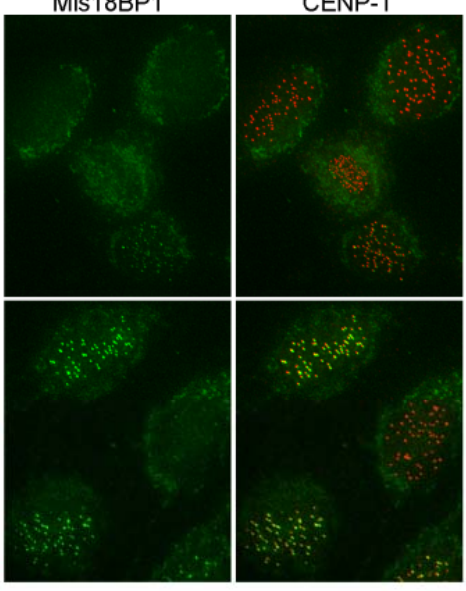

D

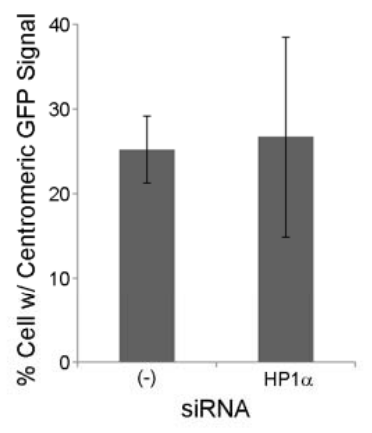

E

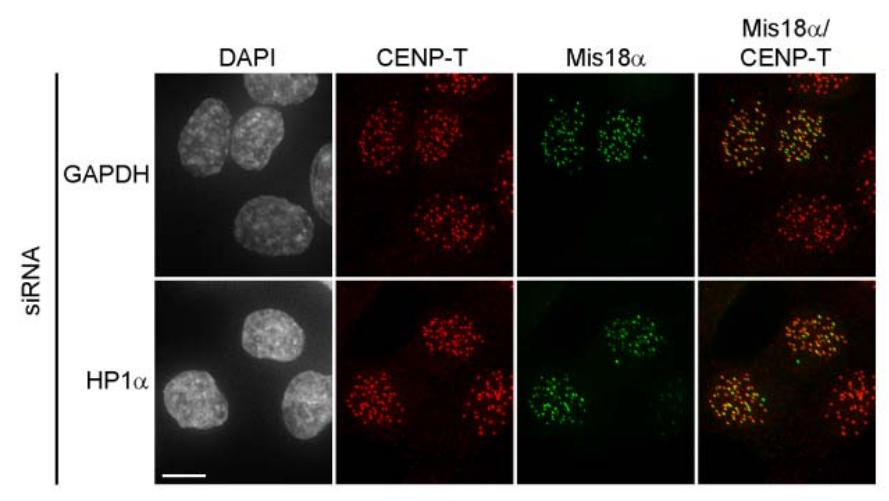

F

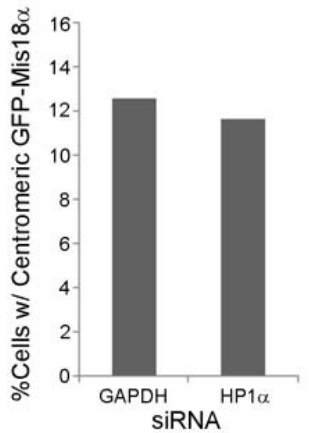

Figure 4-3 


\section{Discussion and future directions:}

It has long been known that pericentromeric heterochromatin is required to maintain genomic stability, and that the Mis 18 complex is required for HJURP-mediated CENP-A deposition. In this section, I determined that the centromeric localization of Mis18BP1 shifts over time from a focal, dot-like appearance to broad and diffuse patches surrounding clusters of centromeres. This staining pattern is reminiscent of HP1 $\alpha$ localization at pericentromeric heterochromatin [190]. HP1 $\alpha$ and HP1 $\beta$ localize to pericentric heterochromatin, and we discovered that Mis $18 \mathrm{BP} 1$ preferentially interacts with HP1 $\alpha$. This interaction is not required for the Mis 18 complex to localize in general to centromeres. However, it does suggest that the Mis 18 complex may perform additional functions at the human centromere outside of the CENP-A deposition pathway. The following proposed experiments seek to explore two aspects of the Mis18BP1-HP1 $\alpha$ interaction. The first set of experiments will focus on obtaining more complete knowledge of the cell cycle regulation regarding the interactions between these two proteins. The other set of experiments will try to determine the downstream functions of the Mis18BP1-HP1 $\alpha$ interaction in centromere biology.

Phospho-regulation of the Mis18BP1-HPla interaction

The data in this section suggests that the Mis18BP1-HP1 $\alpha$ interaction is regulated by the cell cycle. Both proteins contain several highly conserved domains. Mis18BP1 contains a C-terminal SANT domain as well as an N-terminal SANTA domain. HP1 $\alpha$ consists of an $\mathrm{N}$-terminal chromo domain (CD), an internal hinge region and a $\mathrm{C}$-terminal 
chromo shadow domain (CSD). Therefore, the first step to understanding the cell cycle mediated regulation of the Mis18BP1-HP1 $\alpha$ interaction would be to ascertain what portion of each protein is physically interacting with the other.

Both proteins are amenable to recombinant protein preparations, especially when fragmented into smaller portions. Since all fragments of Mis18BP1 that displayed a diffuse centromeric localization pattern contained the SANTA domain, recombinant Mis18BP1 ${ }^{\text {SANTA }}$ will be generated and its ability to interact with the CD, hinge or CSD of HP1 $\alpha$ will be assessed by in vitro pull down experiments. If the required recombinant proteins cannot be generated in sufficient quantities for these experiments, these constructs could also be expressed in human cells. Then the interactions could be assayed by immunoprecipitation. However, that method does not rule out the possibility of additional proteins facilitating the interaction.

Once the interaction domain between Mis18BP1 and HP1 $\alpha$ has been identified, possible phosphorylation sites will be mapped to each fragment. HP1 $\alpha$ is phosphorylated throughout the cell cycle on its N-terminus, but is differentially phosphorylated at its hinge domain in G2/M [191]. Mis18BP1 is phosphorylated by Cdk1 and Cdk2 activity, and has putative phosphorylation sites throughout the length of the protein [164]. Cdk2 kinase activity begins to increases as a cell reaches the end of G1, which corresponds to the timing of the localization shift I observed in the nocodazole release experiment described above (Figure 4-1, A - C) [98]. The Mis18BP1 mutational studies described in chapter 2 indicated that a combination of 24 individual phosphorylation sites were inhibitory to centromeric localization, but those experiments did not delineate between 
several possible combinations of phosphorylations across the entire Mis18BP1 protein [164]. In interphase, Mis18BP1 interacts with chromatin in a highly dynamic way. Therefore, if a hypophosphorylated state originally directed Mis18BP1 to CENP-Acontaining chromatin in early G1; it is possible that during its continual exchange with the centromere, Mis18BP1 may gradually gain specific late G1 phosphorylations, which may shift Mis18BP1 localization within the centromere region, to nearby pericentric heterochromatin.

Additionally, the kinase activity present in the above immunoprecipitations from mitotic extracts may be affecting the post-translational modification status of HP1 $\alpha$. HP1 $\alpha$ also has a highly dynamic interaction profile with the pericentromere, similar to Mis18BP1. In addition, HP1 $\alpha$ is specifically phosphorylated by NDR kinase in G2/M on its hinge domain. This phosphorylation alters HP1 $\alpha$ localization and drives it to the kinetochores [191]. Therefore, the regulation between Mis18BP1 and HP1 $\alpha$ may be regulated by post-translational modifications of either protein.

Once an interaction domain is determined, a series of phospho mutants will be generated in order assess the effects of specific residues on the interaction. Discovering the Mis18BP1-HP1 $\alpha$ interaction domain and possible regulatory posttranslational modifications will be a significant advance in a novel interaction at the human centromere. In addition, gaining the knowledge of how to disrupt the Mis18BP1-HP1 $\alpha$ interaction will also prove invaluable to probe the downstream effects of this proteinprotein interaction. 
Functions of the Mis 18 complex outside of the CENP-A deposition pathway

While HP1 $\alpha$ depletion does not seem to have an effect on the localization of the Mis 18 complex, we have not asked the reciprocal question: Is the Mis18 complex required for the recruitment of $\mathrm{HP} 1 \alpha$ to the centromere region and for the establishment of pericentric heterochromatin? While the GFP-HP1 $\alpha$ cell line constitutively expresses GFP-HP1 $\alpha$, localization of this protein to pericentric heterochromatin is most easily seen in mitotic spreads. Therefore, the first step to determine whether there is a dependency on the Mis 18 complex for HP1 $\alpha$ localization would be to deplete the Mis 18 complex from GFP-HP1 $\alpha$ cells for 48 to 72 hours. After successful reduction of the Mis 18 proteins, HP1 $\alpha$ localization will be determined by immunofluorescence on mitotic spreads. Comparing the presence and intensity of GFP-HP1 $\alpha$ at the centromere region in controls to those cells that were depleted of the Mis 18 complex would allow us to determine if transient Mis 18 depletion affects the binding of HP1 $\alpha$ to the centromere. This would be an interesting and novel linkage between the deposition of CENP-A and the maintenance of pericentric heterochromatin. If there was an effect in HP1 $\alpha$ localization, this would indicate a new function for the Mis 18 complex in the maintenance of centromeric structure.

As the first set of experiments determined the domains that mediate the interaction between Mis18BP1 and HP1 $\alpha$, it will be possible to disrupt the Mis18BP1HP1 $\alpha$ interaction. For example, if a specific phosphorylation event facilitates the interaction, the responsible kinase could be inhibited pharmacologically. In addition, phosphomutants could be generated that no longer mediate the interaction. Once the 
Mis18BP1-HP1 $\alpha$ interaction has been successfully disrupted, then the downstream effects of this particular protein-protein interaction could be assessed.

Disruption of pericentromeric heterochromatin leads to genomic instability, which is characterized by segregation errors. One possible way to assess the effects on genomic stability would be to perform a chromosome loss assay after disruption of the Mis18BP1HP1 $\alpha$ interaction. Stable cell lines expressing a shRNA resistant form of a Mis18BP1HP1 $\alpha$ interaction mutant can easily be generated by the Flp- $\operatorname{In}^{\mathrm{TM}}$ recombination system. Once the stable line is created, viral transfection of an inducible shRNA will yield a cell line that will only express the mutant form of HP1 $\alpha$ or Mis18BP1 when shRNA expression is induced. After successful depletion of the endogenous protein, FISH analysis could be used to compare the copy number of individual chromosomes in control and depleted cells. If the copy number of the marker chromosome changes appreciably when the shRNA is expressed, then the Mis18BP1-HP1 $\alpha$ interaction truly has some effect on chromosome segregation fidelity. The benefit of this particular experiment is that Mis18BP1-HP1 $\alpha$ disruption can be assessed over longer periods of time as compared to transient transfections of exogenous mutant proteins and siRNA. This would be an ideal way to assess the disruption of this interaction over the course of several cell generations rather than a maximum of three generations during a 72-hour transient transfection. 


\section{Additional interactions between Mis18 and the CCAN}

\section{Introduction:}

Current investigations indicate that the recruitment of newly synthesized CENP-A nucleosomes to existing centromeres is mediated by contacts between the deposition machinery and the CCAN. In particular, several studies have detailed physical interactions between the Mis18 complex and CENP-C [99, 100, 109]. However, CENP-C may not be the sole mechanism by which the Mis 18 complex is localized to the centromere. Instead, insights from the literature and the following preliminary data suggest that the Mis 18 complex requires several contact points at the centromere to properly localize and initiate CENP-A deposition.

Several lines of evidence suggest that the CENP-H/I/K complex may play a significant role in Mis18 complex localization. Previous studies highlight the requirement for CENP-I in the CENP-A deposition pathway [22, 192]. In one particular experiment, CENP-I was targeted to chromatin on a chromosome whose endogenous centromere had been removed by recombination, and the tethered CENP-I established CENP-A deposition and a functional kinetochore at that position [192]. CENP-A deposition requires the Mis 18 complex at endogenous centromeres [18, 90, 91], and the functionality of these ectopic, CENP-I derived kinetochores indicates that CENP-A was likely assembled by its proper deposition machinery.

In addition, yeast genetics indicate a relationship between the Mis18 complex and the CENP-H/I/K complex. Scm3 not only requires Mis18 to localize to centromeric chromatin, but the fission yeast homolog of CENP-K (Sim4) is also required for Scm3 
recruitment [156]. Mis18 and Mis16 (RbAp48/46) also appear to be required for Mis6 (CENP-I) localization in S. pombe [91]. These data highlight a conserved mutual interaction between the Mis 18 complex and the CENP-H/I/K complex in the CENP-A deposition pathway. Therefore we sought to explore the interactions between the Mis18 and CENP-H/I/K complexes in human cells, and establish that CENP-C may not be the sole CCAN adapter for the centromeric localization of the human Mis18 complex.

\section{Preliminary results:}

\section{CENP-I and CENP-H recruitment effected by Mis $18 \alpha-M i s 18 \beta$}

The first step to understand the functional interaction between the CENP-H/I/K complex and Mis 18 complex was to deplete components of the Mis18 complex and look for changes in the localization of CENP-I and CENP-H. Since the Mis18 complex is required for CENP-A deposition, HJURP was depleted as an internal control for the reduction in centromeric CENP-A. If a change in the localization of CENP-I or CENP-H was observed when the Mis 18 complex was depleted, but not when HJURP protein levels were reduced, it would indicate a specific dependency on the function of the Mis 18 complex, rather than an effect of a general reduction of CENP-A at the centromere.

First I assessed the effects of the Mis18 complex on CENP-I localization. Mis18ß, Mis18BP1 and HJURP were depleted in U2OS cells by siRNA and compared to cells treated with negative control siRNA. All three protein levels were reduced below $10 \%$ of the control treated cells, while CENP-I levels remained constant across all conditions (Figure 4-4A). Cells were prepared for immunofluorescence and stained for endogenous 
CENP-A and CENP-I. In cells treated with siRNA against the Mis18 complex and HJURP, the intensity of centromeric CENP-A was decreased, further indicating successful depletion of all three proteins. All cells across each treatment condition retained CENP-I staining at the centromere. The intensity of centromeric CENP-I staining was only reduced in the Mis $18 \beta$ condition, and not in cells treated with Mis18BP1 or HJURP siRNA. This reduction was small, but statistically significant. However, centromeric CENP-I levels actually increased when cells were depleted of Mis18BP1. This may suggest that CENP-I localization is specifically dependent on Mis $18 \beta$ rather than the entire Mis18 complex or the full CENP-A deposition machinery. Despite a reduction in centromeric CENP-A in all conditions, CENP-I retained the ability to localize to centromeric chromatin.

A similar experiment was repeated in a cell line that stably expressed GFP-tagged CENP-H. Mis18BP1, Mis18 $\alpha$ and HJURP were depleted and GFP-CENP-H localization in each condition was compared to cells treated with negative control siRNA oligonucleotides. Centromeric CENP-A levels were used to assess the degree of Mis18 complex and HJURP depletion. In all three conditions, the protein level of CENP-A at individual centromeres were greatly reduced. Similar to CENP-I, all imaged cells retained GFP-CENP-H at centromeres regardless of the treatment condition. There was a slight $(<1000 \mathrm{AU})$ but significant decrease in GFP-CENP-H intensity when cells were treated with siRNA against Mis 18 $\alpha$. However, centromeric GFP-CENP-H levels actually increased when cells were depleted of Mis18BP1 or HJURP. This may suggest that CENP-H localization is specifically dependent on Mis18 $\alpha$ rather than the entire Mis18 
complex, which was similar to what was seen with Mis $18 \beta$ for CENP-I. These results may indicate additional interactions and functions that are specifically related to only a subset of the Mis18 complex proteins. 


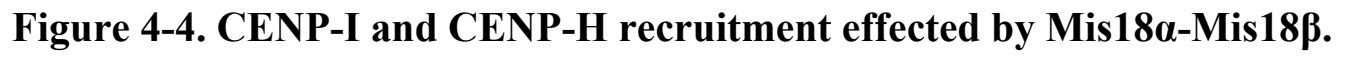

(A) Western blots of whole cell lysates from U2OS cells treated with siRNA against Mis18BP1 and Mis18ß (left blot) as well as HJURP (right blot) for $48 \mathrm{~h}$. Standard curve was generated with lysates from negative control treated cells. Ponceau staining was used as a loading control. (B) Representative images of U2OS cells treated as in A. CENP-A and CENP-I were stained with antibodies against the endogenous proteins. (C) Graphs of the integrated intensities of CENP-A (upper graph) and CENP-I (lower graph) staining at individual centromeres, for each siRNA condition. Red interval bars indicate the $95 \%$ confidence interval around the mean. Red $* * *$ indicates a $\mathrm{p}$-value $<0.001$ calculated by a Mann-Whitney test. $\mathrm{N}=1$. (D) Representative images of cells stably expressing GFPCENP-H treated with siRNA against Mis18BP1, Mis18 $\alpha$ and HJURP for 48 h. CENP-A was stained with antibodies against the endogenous protein. (E) Graphs of the integrated intensities of CENP-A staining (upper graph) and GFP-CENP-H signal (lower graph) at individual centromeres, for each siRNA condition. Red interval bars indicate the 95\% confidence interval around the mean. Red $* * *$ indicates a p-value $<0.001$ and $* *$ indicates a $\mathrm{p}$-value of $<0.01$ as calculated by a Mann-Whitney test. $\mathrm{N}=1$. 


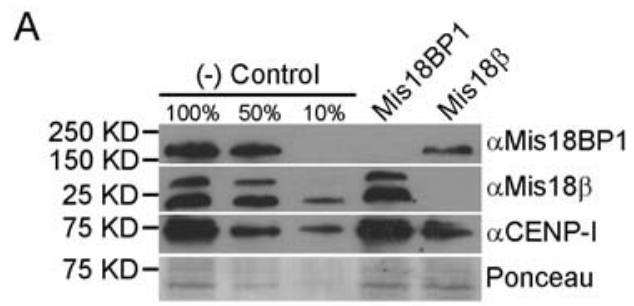

B

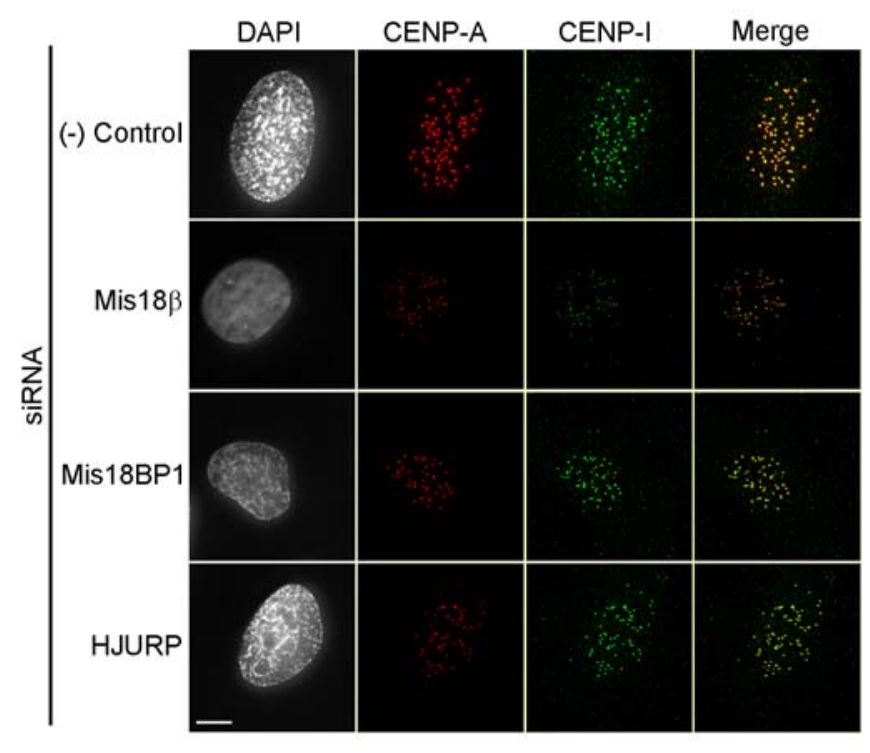

D

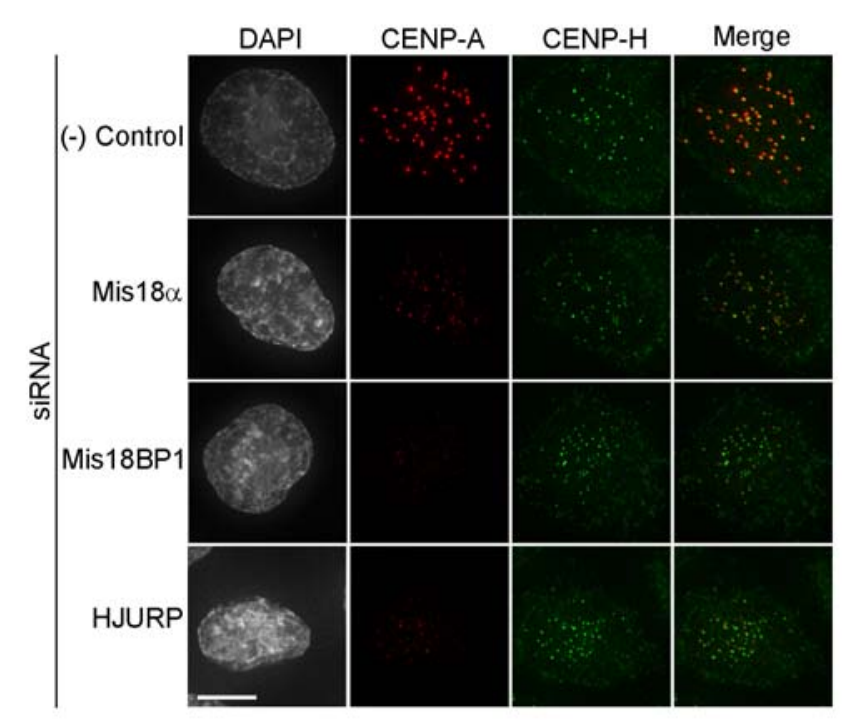

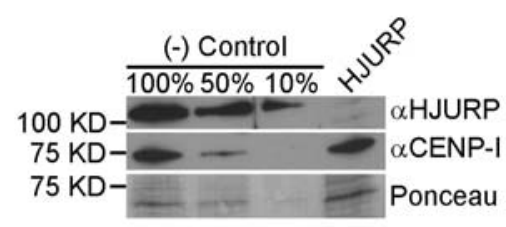

C
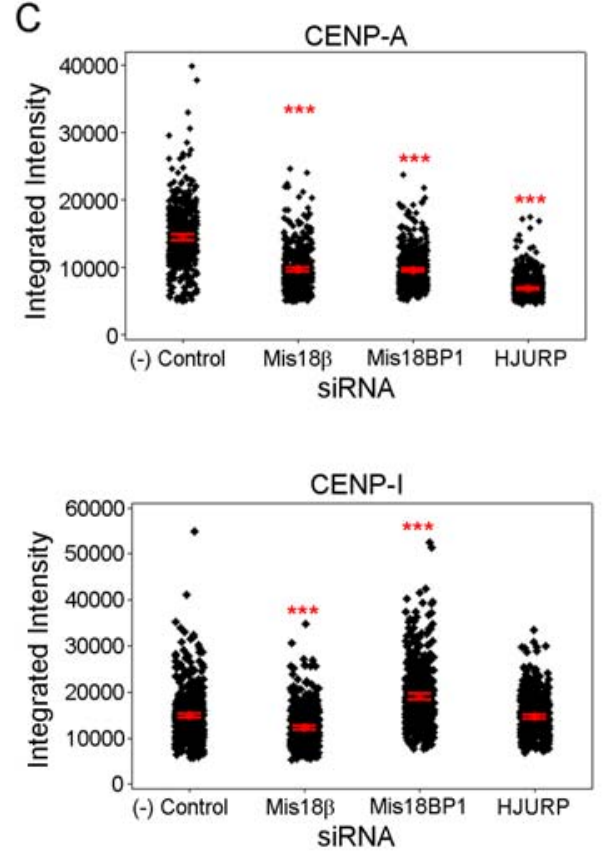

E
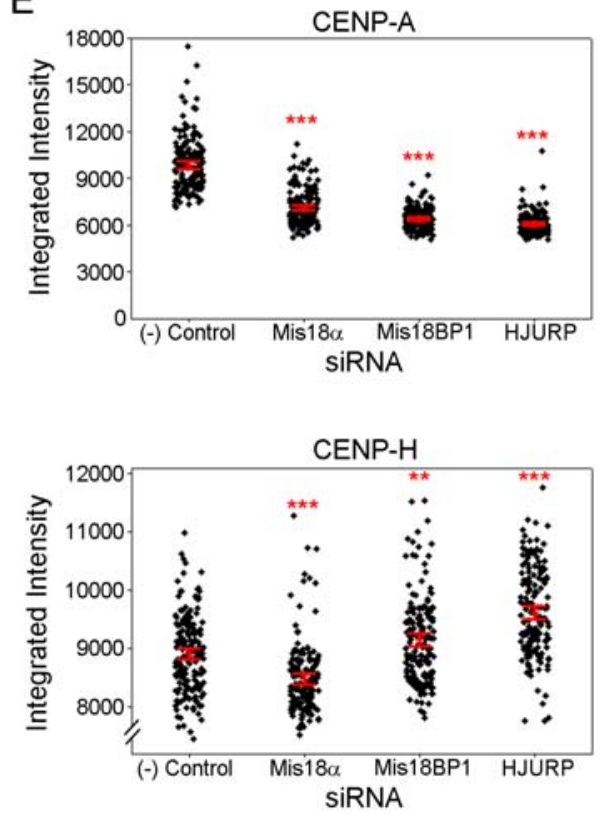

Figure 4-4 
CENP-I and CENP-K depletions reduce Mis18a localization in interphase

In the reciprocal experiment, HeLa cells that stably express GFP-Mis $18 \alpha$ were treated with siRNA pools against CENP-I and CENP-K. After 48 hours of treatment, live cells were imaged by phase to determine the mitotic index. The same cells were then fixed and stained for endogenous CENP-A and CENP-I. As expected CENP-I levels at the centromere decreased when cells were depleted of CENP-I and CENP-K (Figure 4-5, A and B). In addition, depleting either CENP-I or CENP-K resulted in a clear increase in the mitotic index, which is indicative of the loss of both proteins from the centromere (Figure 4-5C) [22]. Since the Mis18 complex does not localize to centromeres during mitosis, this cell cycle arrest decreased the number of cells that were eligible to recruit GFP-Mis $18 \alpha$ to centromeric chromatin. A decrease in the percentage of total cells that localized GFP-Mis $18 \alpha$ to centromeres may not indicate a direct dependency on the presence of CENP-I or CENP-K for Mis 18 complex recruitment. Therefore, I only assessed the GFP-Mis $18 \alpha$ localization in cells that did not have a mitotic phenotype.

Of the remaining non-mitotic population, there was a marked decrease in the percentage of cells that recruited GFP-Mis $18 \alpha$ to the centromere. Centromere intensity analysis of cells that had centromeric GFP-Mis $18 \alpha$ showed a significant decrease in CENP-I intensity. This indicates that these cells were also targeted by the CENP-I and CENP-K siRNA and likely were not a population that escaped depletion. Together with the experiments highlighted in Figure 4-4, these data indicate the possibility of a mutual dependency between CENP-H/I/K and Mis18 $\alpha-M i s 18 \beta$ for proper centromere localization. 
Figure 4-5. CENP-I and CENP-K depletions reduce Mis18 localization in interphase.

(A) Graph of the integrated CENP-I intensity at individual centromeres under each siRNA condition. Red interval bars indicate the $95 \%$ confidence interval around the mean. Red $* * *$ indicates a $\mathrm{p}$-value $<0.001$ calculated by a Mann-Whitney test. $\mathrm{N}=1$. (B) Graph showing the percentage of total cells that are in mitosis. $N=1$. (C) Representative images of cells stably expressing GFP-Mis $18 \alpha$ treated with either negative control siRNA or siRNAs against CENP-I and CENP-K. CENP-A and CENP-I were stained with antibodies against the endogenous proteins. Insets show the localization of each protein at an individual centromere. Scale bar $=5 \mu \mathrm{m}$. (D) Graph depicting the percentage of interphase cells that had GFP-Mis $18 \alpha$ localized to CENP-A foci. $N=1$. (E) Graph of the integrated centromeric CENP-I intensity in cells that have GFP-Mis18 $\alpha$ at centromeres.

Red interval bars indicate the $95 \%$ confidence interval around the mean. Red $* * *$ indicates a $\mathrm{p}$-value $<0.001$ calculated by a Mann-Whitney test. $\mathrm{N}=1$. 
A

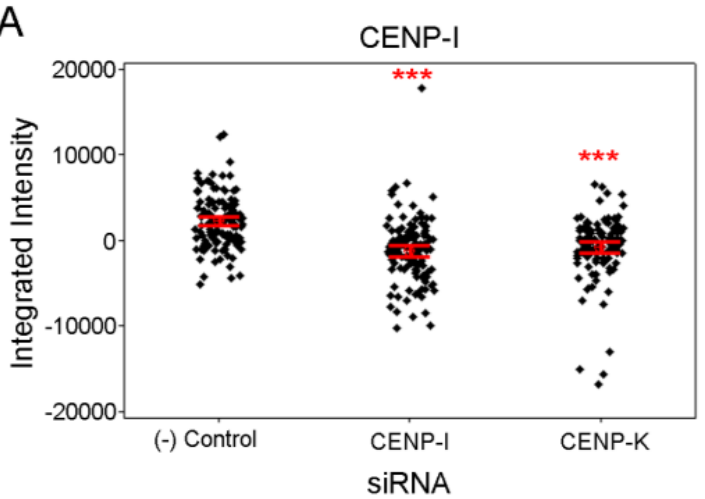

B

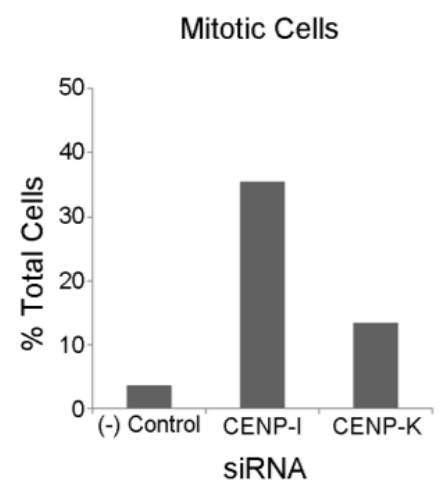

C
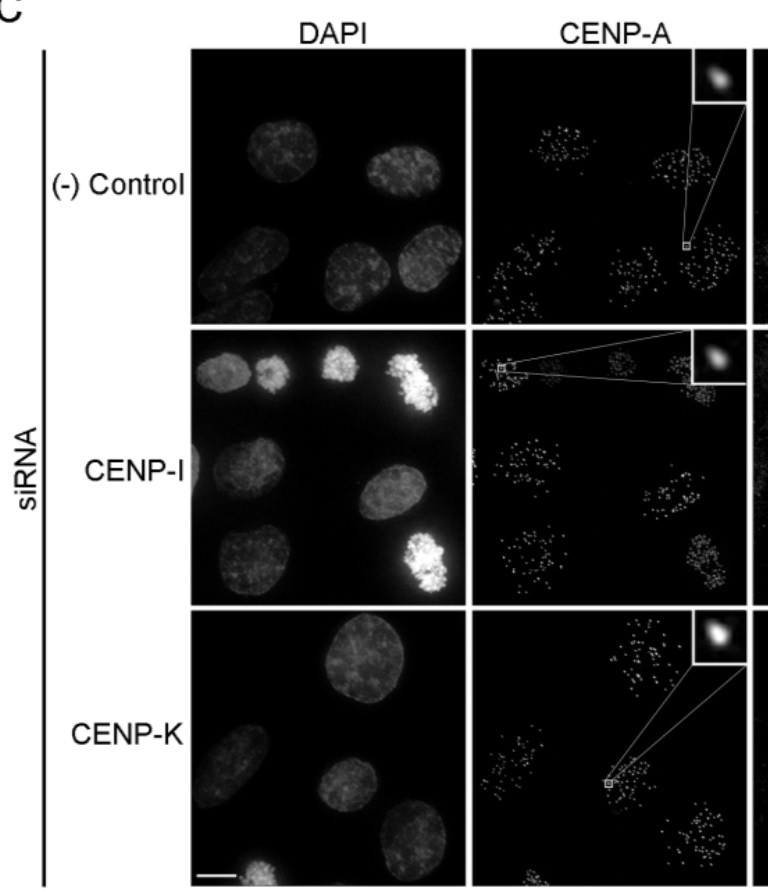

CENP-I

GFP-Mis $18 \alpha$

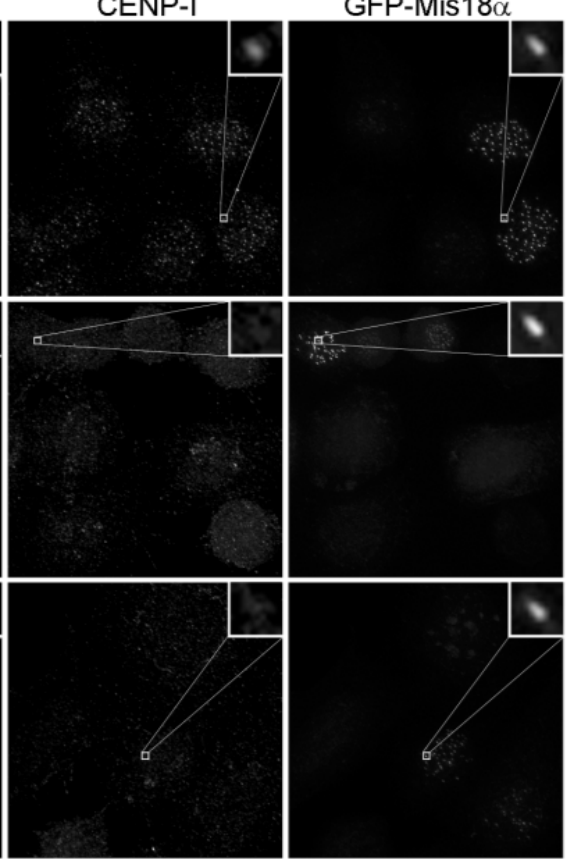

D

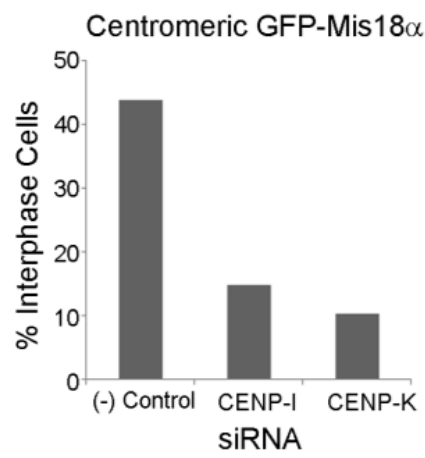

E

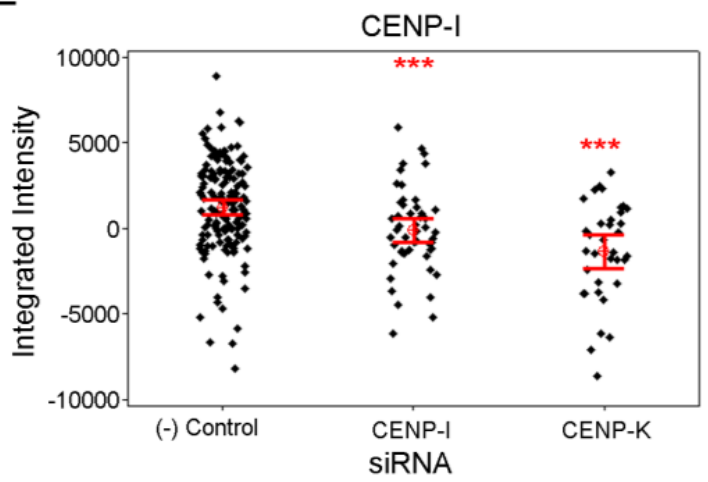

Figure 4-5 
CENP-C is not required to maintain Mis $18 \alpha$ at the centromere

Previously described experiments in chapter 3 and work performed in other laboratories have shown a definite relationship between CENP-C and the Mis 18 complex $[99,100,109]$. Xenopus has two isoforms of Mis18BP1. One isoform is recruited throughout the cell cycle (M18BP1-1), while the other isoform localizes only during G1 (M18BP1-2), similar to the localization pattern of most Mis18BP1 homologs [100]. In extracts depleted of CENP-C, only the metaphase localization of M18BP1-1 was compromised, while the canonical, G1 localization of both isoforms remained intact [100]. Additionally, CENP-C depletions by shRNA in mice only weakly reduced the localization of Mis18BP1 to centromeres, despite a significant reduction in CENP-C protein levels [99]. Therefore, the model, in which CENP-C serves as the sole contact point between the Mis18 complex and centromeric chromatin, does not fully describe the data.

In order to gain a deeper understanding of the role CENP-C plays in facilitating the centromeric localization of the Mis18 complex, I sought an assay were I could determine if CENP-C was required to keep the Mis 18 complex localized to centromeres, after the complex had already been loaded onto chromatin. Perpelescu, et al, described a high salt wash assay that diminished the presence of CENP-C at endogenous centromeres by immunofluorescence, while maintaining the presence of other centromere proteins that are more tightly associated with chromatin, such as CENP-A [132]. If a loss of CENP-C after the high salt wash resulted in a diminished presence of the Mis 18 complex at centromeres, then CENP-C may play a role in continually tethering the Mis18 complex to 
the centromere. However, if the Mis18 complex remained associated with the centromere despite a loss of CENP-C, then CENP-C would likely not be the main binding site for the Mis 18 complex at the centromere.

HeLa cells stably expressing GFP-Mis18 $\alpha$ were grown on glass coverslips for 48 hours to $75 \%$ confluence. The growth media was then removed and replaced with either PBS or PBS supplemented with $500 \mathrm{mM} \mathrm{NaCl}$ for 30 minutes at $37^{\circ} \mathrm{C}$. After the low and high salt washes, the GFP-Mis18 $\alpha$ expressing cells were fixed and stained for CENP-C (Figure 4-6A). After high salt extraction, centromeric CENP-C was clearly depleted but there was no change in the percentage of cells that localized GFP-Mis $18 \alpha$ to centromeres (Figure 4-6, A and B). In order to ensure that the cells that had GFP-Mis18 $\alpha$ localized to centromeres were indeed depleted for centromeric CENP-C; I assessed the GFP-Mis18 $\alpha$ and CENP-C nuclear intensities in GFP-Mis18 $\alpha$ positive cell population (Figure 4-6C). The GFP-Mis18 $\alpha$ positive cells that had been washed with high salt clearly displayed a reduction in centromeric CENP-C (Figure 4-6, A and C).

Since CENP-C localization was determined using an antibody to the endogenous protein, I needed to ensure that the reduction in centromeric CENP-C was not a result of the CENP-C antibody's inability to recognize the antigen after exposure to the high salt wash. In order to answer this question, I performed the salt wash experiment on GFPMis18 $\alpha$ expressing cells that had already been fixed with formaldehyde prior to the salt wash and compared the nuclear intensity of CENP-C staining to the original protocol (Figure 4-6, D and E). Nuclear intensities of GFP-Mis18 $\alpha$ and CENP-C were little changed when the salt wash occurred after fixation (Post-Fix conditions). This indicates 
that the CENP-C antibody retained its ability to recognize the antigen despite the cell's exposure to high salt conditions. However, the decrease in nuclear CENP-C was once again seen when cells were washed with high salt prior to fixation, similar to the original salt wash experiment (Figure 4-6, A - C). Therefore, the depletion of CENP-C and the retention of GFP-Mis1 $8 \alpha$ at the centromeres under high salt conditions were not due to an experimental artifact.

These data indicate that CENP-C is not required for the Mis18 complex to remain associated with centromeric chromatin. While previous data indicate a role for CENP-C in the recruitment of the Mis18 complex to centromeres in several organisms, CENP-C is not continually required for the Mis 18 complex to remain at the human centromere. Additional interactions between the Mis 18 complex and centromeric chromatin or the CCAN must exist. 
Figure 4-6. CENP-C is not required to maintain Mis18 $\alpha$ at the centromere.

(A) Representative images of HeLa GFP-Mis $18 \alpha$ cells that were washed with low salt (PBS) or high salt (PBS $+500 \mathrm{mM} \mathrm{NaCl}$ ) prior to fixation. Centromeric CENP-C was visualized with an antibody raised against the endogenous protein. Scale bar $=5 \mu \mathrm{m}$. (B) Graph depicting the percentage of nuclei that had GFP-Mis18 $\alpha$ localized to centromeres. At least 95 cells were analyzed in each condition. Error bars display the standard deviation across replicates. $\mathrm{N}=2$. (C) Bar graph shows the mean nuclear intensity of GFP-Mis 18 $\alpha$ (green) and CENP-C (red) in GFP-Mis18 $\alpha$ positive nuclei in both salt wash conditions. Nuclear intensities were normalized to 1 in the PBS condition. Error bars show the standard deviation between replicates. The Student's t-test was used to determine statistical significance $(\mathrm{p}<0.05)$. $\mathrm{N}=2$. (D) and $(\mathrm{E})$ Graphs depict the mean nuclear intensity of cells subjected to the low and high salt washes before (Pre-Fix) and after (Post-Fix) formaldehyde fixation of GFP-Mis18 $\alpha$ and CENP-C respectively. $\mathrm{N}=1$. 

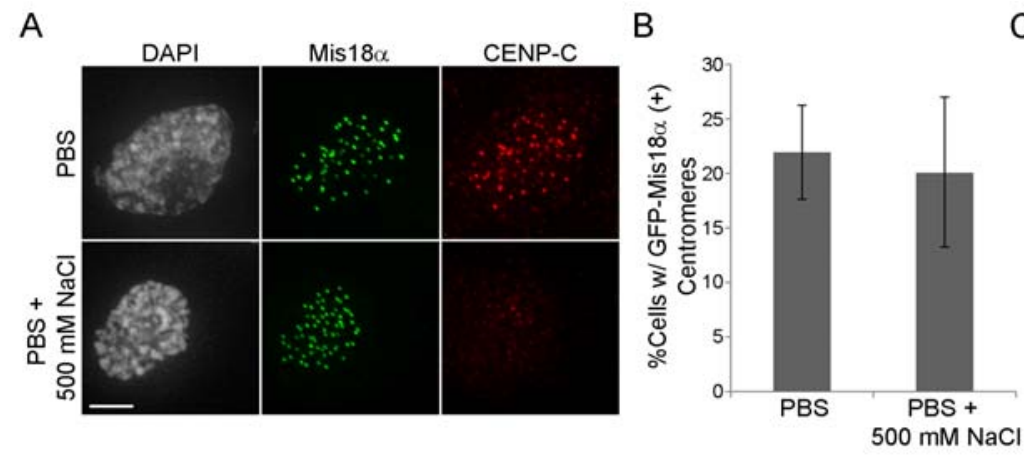

C
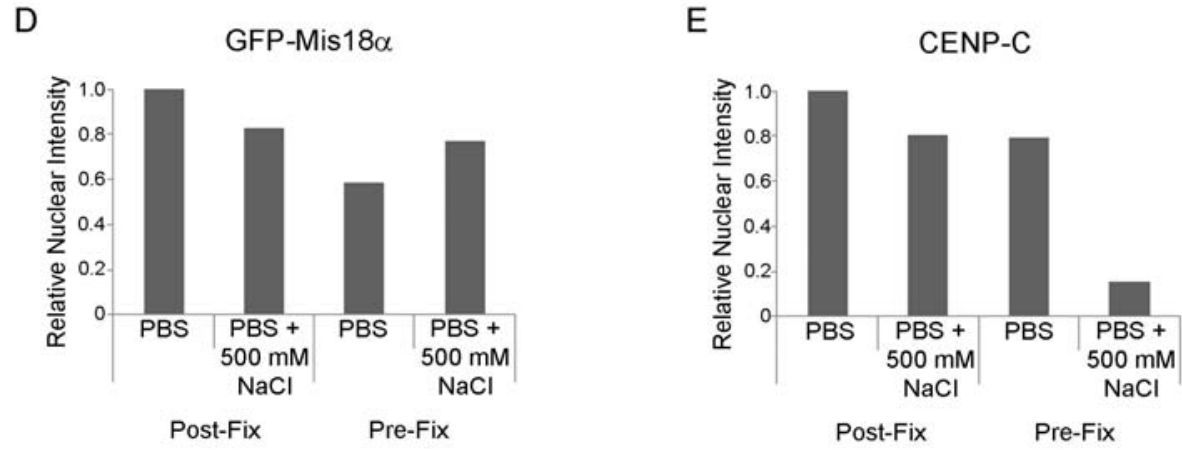

Figure 4-6 


\section{Discussion and future directions:}

While CENP-C may be required for the initial localization of the Mis 18 complex, it is dispensable for maintaining the complex's association with the centromere throughout G1. CENP-C is unlikely the sole binding partner of the Mis 18 complex at the human centromere. In addition to the interaction between Mis18BP1 and CENP-C, Mis $18 \alpha$ and Mis $18 \beta$ appear to functionally interact with the CENP-H/I/K complex. From these preliminary results and previous genetic and cell biological studies, CENP-A deposition appears to be determined by several contacts between the proteins of the CCAN and the Mis 18 complex. However, there are several additional experiments that are required to fully support this hypothesis.

In order to fully ascertain the mutual dependency between the Mis 18 complex and CENP-H/I/K, the issue of cell cycle arrest needs to be addressed. CENP-I and CENP-K depletions yield a significant mitotic arrest phenotype. The Mis 18 complex is present at centromeres only in G1. While centromeric analysis of CENP-I intensity at the few remaining GFP-Mis18 $\alpha$ positive centromeres in CENP-I and CENP-K depleted cells indicated that these cells did not escape siRNA depletion, the dearth of Mis $18 \alpha$ positive cells may still simply be a result of a perturbed cell cycle. In order to combat the cell cycle effects of CENP-I and CENP-K depletions, I propose the following experiments.

The Mis $18 \mathrm{BP}^{24 \mathrm{~A}}$ mutant described in chapter 2 localizes to centromeres outside of G1 and can be seen at mitotic centromeres [164]. Therefore, transfecting in GFPMis $18 \mathrm{BP}^{24 \mathrm{~A}}$ while depleting the CENP-H/I/K complex would allow us to assess Mis 18 complex recruitment regardless of cell cycle position. Additionally, we could force 
CENP-I and CENP-K depleted cells to reenter the cell cycle by perturbing the spindle assembly checkpoint (SAC). Pharmacological interventions could be used, include treating CENP-I and CENP-K depleted cells with an Aurora kinase inhibitor, such as ZM447439. The SAC could also be compromised by codepletion of BubR1, which would also allow the CENP-I and CENP-K siRNA treated cells to escape the mitotic arrest. As GFP-Mis $18 \alpha$ localizes to centromeres in late anaphase, these methods would yield a large population of cells that should have GFP-Mis18 $\alpha$ localized to centromeric chromatin. Therefore, any affect would be easily discernable. Both of these methods will allow us to confirm the requirement for CENP-H/I/K in Mis 18 localization without the possible confounding nature of cell cycle regulation.

Previous work has shown that targeting CENP-I to chromatin can recruit CENP-A and generate a functional centromere. As canonical CENP-A deposition requires the Mis18 complex, it is hypothesized that this non-centromeric deposition is mediated through the normal pathway. Therefore, assessing whether CENP-H/I/K is sufficient to recruit the Mis 18 complex to chromatin can also confirm that CENP-I mediated CENP-A deposition occurs through the Mis18 complex rather than through other chromatin assembly factors [193].

If the Mis 18 complex localizes to the ectopic CENP-I foci, it will be important to understand which member of the Mis 18 complex is mediating that interaction. As detailed in chapter 2 and 3, the proteins of the Mis 18 complex have each evolved to interact with unique binding partners at the centromere. In addition, the Mis18 $\alpha$ - and Mis18 $\beta$-specific effects on CENP-H and CENP-I recruitment, while slight, suggest that 
this theme may also be true in this pathway. The LacO array could be used to determine this information; however, the presence of the endogenous Mis18 complex proteins would complicate the analysis. Therefore, I propose to attempt to isolate recombinant proteins or fragments of the CENP-H///K complex in order to assess whether there are physical interactions between particular Mis 18 complex proteins and individual members of the CENP-H/I/K complex.

However, the most powerful use of the $\mathrm{LacO}$ technology would be to assess the efficiency of CENP-A deposition at the LacO array when the CENP-H/I/K complex or CENP-C is targeted to the array. Comparing the efficiency of CENP-A recruitment when the Mis 18 complex is targeted by the CENP-H/I/K, CENP-C or a combination of both would yield important insights as to sufficiency of either interaction. 


\section{Possible histone binding capabilities of Mis18BP1}

\section{Introduction:}

Human centromeres, and the centromeres of all known Mis18BP1-containing organisms, are regional centromeres whose location is specified epigenetically. Mis18 binding protein 1 (Mis 18BP1, M18BP1 or KNL-2) is a conserved protein in higher eukaryotes. Mis18BP1 has two domains, a C-terminal SANT domain and a more Nterminally located SANT-associated (SANTA) domain. The SANT domain, which lies outside of the essential portion of Mis18BP1 (amino acids 2-721), is extremely well conserved (Figure 4-7A).

SANT domains are found in many chromatin remodeling and modifying complexes. SANT domains and the highly related Myb domain, which is the DNA binding domain originally found in c-Myb, have been found to have a variety of functions $[112,194]$. They have been known to function as interaction domains between complex partners, histone binding modules, and DNA recognition motifs. While these functions may seem very divergent, a common theme is the fact that SANT domains mediate specific interactions that drive localization and or activation of protein complexes in the context of chromatin.

A subcellular fractionation protocol allowed us to determine the localization of Mis18BP1 in different portions of a cell. Blotting the fractions for endogenous Mis18BP1 showed that in randomly cycling cells, Mis18BP1 is overwhelmingly found in the chromatin fraction rather than in the nucleoplasm as evidenced by the lack of nucleophosmin (Figure 4-7B). This agrees with previous experiments that have isolated 
the Mis18 complex from chromatin preparations as well as the fact that Mis18BP1 remains bound to chromatin throughout the majority of G1 [91, 133]. Although the SANT domain is not required for CENP-A deposition, it could still mediate chromatinbinding activity of Mis18BP1, outside of the CENP-A deposition pathway. Therefore, I was interested in testing whether the conserved SANT domain of Mis18BP1 could interact in vitro with the various histone tails found in centromeric and pericentric chromatin. 
Figure 4-7. Mis18BP1 contains a highly conserved SANT domain.

(A) Alignment of SANT domains from Mis18BP1 homolog across evolution. Clustal W2 alignment of C-terminal SANT domains from evolutionarily diverse Mis18BP1

homologs using Geneious Pro software [154]. Shading indicates conservation. Amino acid numbers correspond to the human protein sequence. (B) Subcellular fractionation from randomly cycling cells. Anti-Mis18BP1 western blot indicates the majority of Mis18BP1 lies in the chromatin fraction. Anti-nucleophosmin antibody was used to indicate proper fractionation of the soluble nucleoplasm from the chromatin fraction.

A
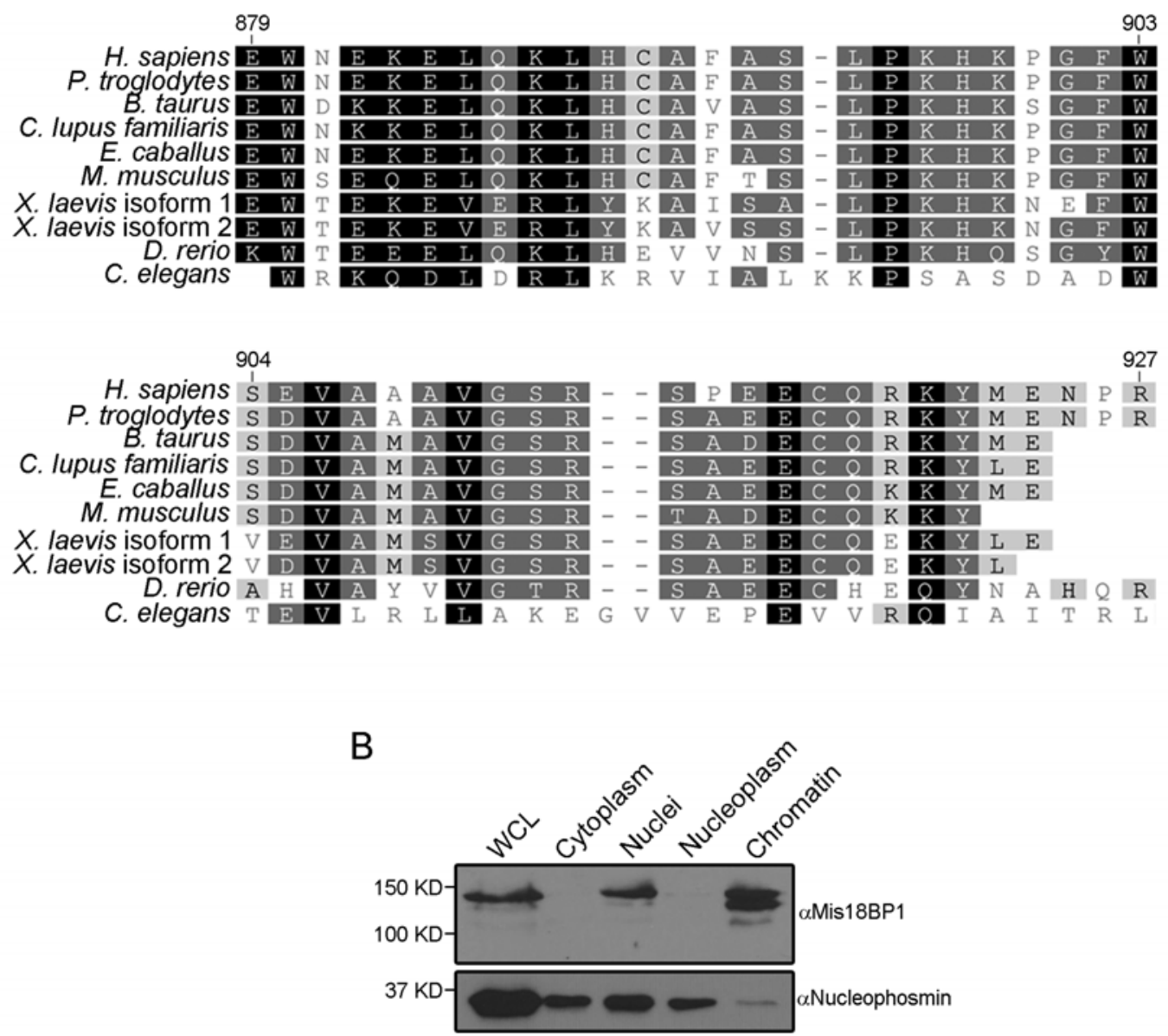

Figure 4-7 


\section{Preliminary results:}

Mis18BP1 SANT domain is not sufficient for in vitro histone tail binding

The epigenetic environment of the human centromeres is a complex mixture of canonical H3 containing nucleosomes as well as CENP-A-containing nucleosomes. Therefore, there is the possibility of bindings all major histone variants at the human centromere. In order to ascertain if the SANT domain of human Mis18BP1 had any affinity for unmodified histone tails, we used a GST pull down strategy.

Glutathione S-transferase (GST) fusions to the N-termini of H2A, H2B and CENP-A were constructed in the lab, while GST-H3.1, GST-H3.3 and GST-H4 were a gift from Dr. Patrick Grant $[195,196]$. In addition, the SANT domain from Mis18BP1 was tagged N-terminally with a $6 \mathrm{xHis} /$ thrombin/S-tag $\left(\right.$ His-Mis $18 \mathrm{BP} 1^{\text {SANT }}$ ). The GSTtagged histone tails and the His-Mis18BP $1^{\text {SANT }}$ construct were purified from bacteria to milligram per milliliter concentrations (Figure 4-8A).

After purification, an equal amount of each GST-histone tail was incubated with the purified His-Mis18BP1 ${ }^{\mathrm{SANT}}$ and binding was assessed by GST pull down. Two different protocols were used to test the interaction. In the first, Pre-Mix protocol, each

GST-histone tail fusion protein was mixed with equal amounts of His-Mis18BP1 ${ }^{\text {SANT }}$ for three hours prior to GST pull down. The second protocol, Pre-Bound protocol, the GSThistone tail fusions were first bound to the immobilized glutathione agarose. Then the His-Mis18BP $1^{\text {SANT }}$ protein was allowed to interact with the pre-bound agarose. The PreBound protocol was used to ensure that all GST-histone tails that were present in the 
binding reaction had already made a sufficient interaction with the agarose, possibly slightly increasing the sensitivity of the assay.

However, we observed no binding between the His-Mis18BP1 ${ }^{\text {SANT }}$ construct and any of the GST-histone tail fusions (Figure 4-8, B and C). Coomassie staining clearly showed the presence of the His-Mis $18 \mathrm{BP} 1^{\text {SANT }}$ as well as the GST fusions in the input. In addition, all GST-histone tail fusions were found solely in the bound fractions of the GST pull downs. This indicates that the pull down was very efficient, leaving little GST protein unbound. However, I only found His-Mis $18 \mathrm{BP} 1^{\text {SANT }}$ in the unbound fraction in every case. In addition, I used an anti-His antibody to further confirm the lack of HisMis18BP $1^{\text {SANT }}$ in the bound fraction of the GST pull downs. His-tag signal was only found in the unbound fractions, and there was no signal even at extended exposure times (Figure 4-8, B and C). 
Figure 4-8. Mis18BP1 SANT domain is not sufficient for in vitro histone tail binding.

(A) Coomassie stained gels of Ni-NTA and immobilized glutathione agarose purified His-Mis18BP $1^{\text {SANT }}$ and GST-histone tail fusion proteins, respectively. BSA was used to generate a standard curve in order to ascertain protein concentration of each purified construct. (B) GST pull down using the "Pre-Mix" protocol where GST-histone tails were first incubated with His-Mis18BP1 ${ }^{\text {SANT }}$ prior binding to the glutathione agarose. Upper panels are coomassie stained gels. The input fractions are on the left, and the bound and unbound fractions from the GST pull downs are on the right. U and B stand for unbound and bound respectively. Bottom right panel is an anti-His western blot of the unbound (left) and bound (right) fractions from the GST pull downs above. (C) Same as in B, using the "Pre-Bound" protocol of pre-incubating the GST-histone tails to the glutathione agarose prior to addition of His-Mis18BP $1^{\text {SANT }}$. 
A
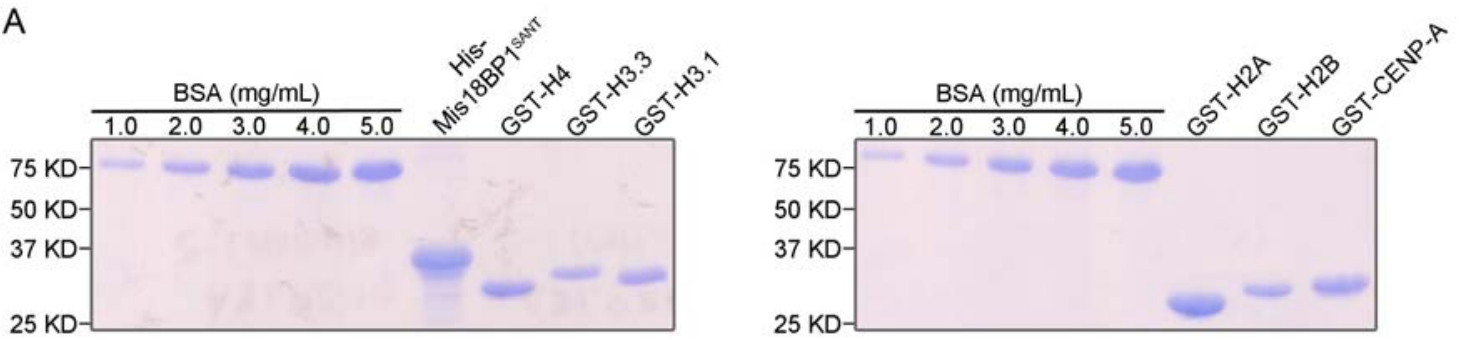

B

\section{Pre-Mix}

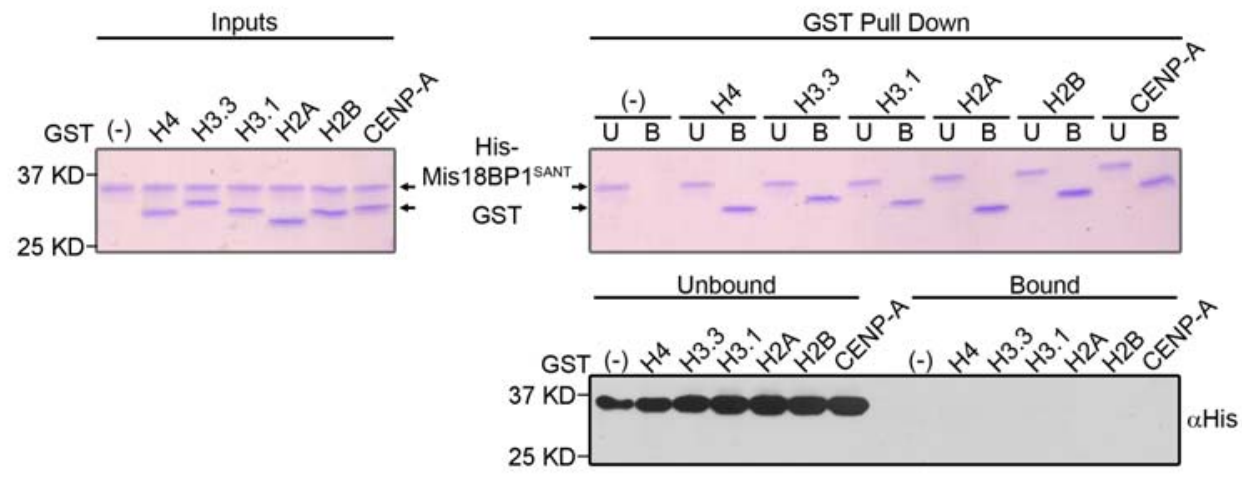

C

\section{Pre-Bound}

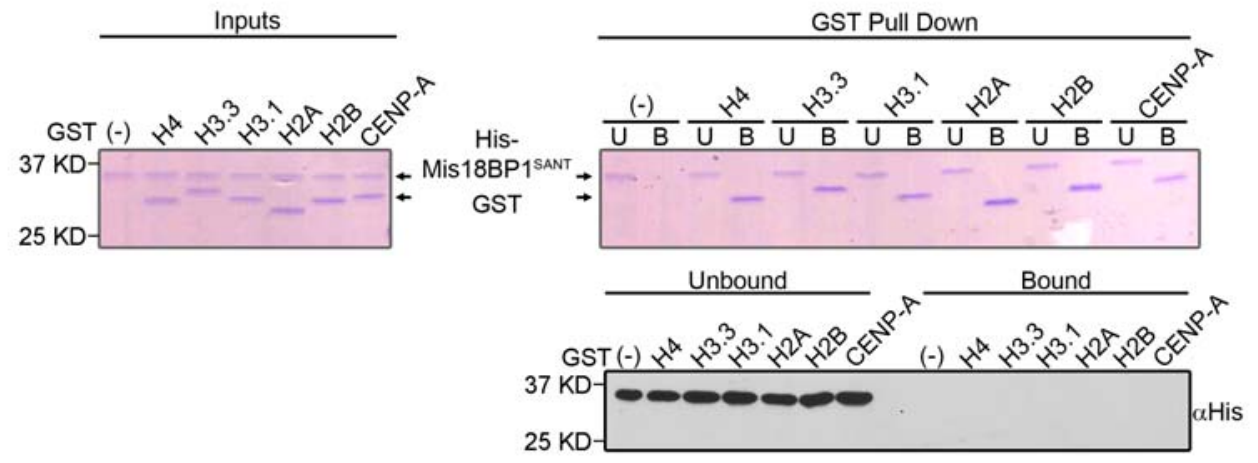

Figure 4-8 


\section{Discussion and future directions:}

While these experiments indicate no physical binding between the HisMis18BP $1^{\text {SANT }}$ construct and the GST-histone tail fusions, there are several limitations of these experiments. A lack of binding between the SANT domain of human Mis18BP1and recombinant histone tails in vitro does not necessarily dictate that the SANT domain has no contact with centromeric histones in vivo. At actual centromeric chromatin, almost all histone tails are subject to some post-translational modification. However, in this experiment, none of those modifications are present. While most SANT domains prefer to interact with unmodified histone tails, there have been a few examples of SANT/Myb domains that bind preferentially to modified peptides [194].

In addition, the SANT domain of human Mis18BP1 may also need to be posttranslationally modified in order to interact with centromeric histone tails. It is known that Mis18BP1 can be phosphorylated by Cdk, and a Cdk site has been mapped directly to the SANT domain of Mis18BP1 [164]. While global Mis18BP1 phosphorylation has been shown to be a negative regulator of centromere interaction, it is possible that single or small combinations of phosphorylation events on Mis18BP1 may actually allow for chromatin binding, while others are inhibitory.

While the SANT domain of Mis18BP1 is dispensable for the centromeric localization and CENP-A deposition function of Mis18BP1, it still may be involved in histone tail interactions for other unknown functions at the centromere. Centromeric chromatin, as well as pericentric heterochromatin, contains a variety of unique histone mark combinations. Therefore I proposed to use the APEX system in order to get a better 
picture of the chromatin to which the Mis18 complex binds in vivo. E. Zasadzinska has generated stable cell lines that express HA-CENP-APEX and HA-H3.1-APEX. In these systems, the APEX-tagged histones are able to modify nearby proteins with a covalent linkage. The active radius of these conjugating enzymes is small and indicates a close interaction between the modified protein and the enzyme tag. Since human centromeres contain alternating blocks of $\mathrm{H} 3$ and CENP-A containing nucleosomes, it will be interesting to see whether the Mis18 complex associates more closely with CENP-A rich regions or prefers $\mathrm{H} 3$ domains as it recruits HJURP for CENP-A deposition.

Additionally, the covalent reaction takes place over the course of seconds once treated with the activator $\left(\mathrm{H}_{2} \mathrm{O}_{2}\right)$. This may allow us to follow possible chromatin contact changes as the localization of Mis18BP1 shifts as cells progress from CENP-A deposition towards the G1/S boundary. 


\section{Materials and Methods}

\section{Mis18BP1-HP1 $\alpha$ interaction:}

\section{Tissue culture}

HeLa derived cells lines were cultured in DMEM High Glucose (Life Technologies) supplemented with 10\% heat inactivated FBS (Optima, Atlanta Biologicals) and 1X Penicillin/Streptomycin (Life Technologies). U2OS derived cell lines were cultured in DMEM High Glucose GlutaMAX ${ }^{\mathrm{TM}}$ (Life Technologies)

supplemented with 10\% FBS and 1X Penicillin/Streptomycin. Cells were incubated at $37^{\circ} \mathrm{C}$ in $5 \% \mathrm{CO}_{2}$ and $85 \%$ humidity. A stable GFP-Mis $18 \mathrm{BP} 1$ expressing cell line was generated using the Flp-In system (Life Technologies) in a previously established HeLa T-REX Flp-In cell lines (S. Taylor, University of Manchester), and maintained in 200 $\mu \mathrm{g} / \mathrm{mL}$ Hygromycin B-supplemented media.

\section{Immunofluorescence preparation and staining protocol}

Cells grown on poly-lysine coverslips were fixed for $10 \mathrm{~min}$ with $37^{\circ} \mathrm{C} 4 \%$ formaldehyde in PBS (137 mM NaCl, $\left.2.7 \mathrm{mM} \mathrm{KCl,} 5.4 \mathrm{mM} \mathrm{Na}_{2} \mathrm{HPO}_{4}, 1.8 \mathrm{mM} \mathrm{KH}_{2} \mathrm{PO}_{4}\right)$ and then quenched for 5 min with $100 \mathrm{mM}$ Tris $\mathrm{pH}$ 7.5. Coverslips were blocked for $1 \mathrm{~h}$ in blocking buffer: $2 \%$ heat inactivated FBS, $2 \%$ BSA, and $0.1 \%$ Triton-X100 in PBS. Primary antibodies were incubated for $1 \mathrm{~h}$ in blocking buffer: anti-CENP-A mouse monoclonal antibody (ab13939, Abcam) at $1 \mu \mathrm{g} / \mathrm{mL}$ and anti-CENP-T rabbit polyclonal antibody serum (D. Cleveland, Ludwig Institute for Cancer Research) at 1:2000, and antiHP1 $\alpha$ (05-689, clone 15.19s2, Millipore) at $10 \mu \mathrm{g} / \mathrm{mL}$. Coverslips were washed three 
times for 5 min with $0.1 \%$ Triton-X100 in PBS. Secondary antibodies were diluted in blocking buffer and were incubated with the coverslips for $1 \mathrm{~h}$ (Alexa-647 conjugated goat-anti-mouse at $0.375 \mu \mathrm{g} / \mathrm{mL}, \mathrm{Cy} 5$ conjugated goat-anti-rabbit conjugated at 0.375 $\mu \mathrm{g} / \mathrm{mL}$, Cy3 conjugated goat-anti-mouse at $0.75 \mu \mathrm{g} / \mathrm{mL}$; Jackson ImmunoResearch Laboratories, Inc.). Cells were washed as above and then incubated in PBS containing 0.2 $\mu \mathrm{g} / \mathrm{mL}$ of DAPI for $5 \mathrm{~min}$. Coverslips were rinsed with PBS prior to mounting onto glass slides with Prolong Gold Antifade Reagent (Life Technologies).

\section{Nocodazole release and image analysis}

HeLa GFP-Mis18BP1 cells were treated with $100 \mathrm{ng} / \mathrm{mL}$ nocodazole in DMSO for $12-14 \mathrm{~h}$. Mitotics were collected and pelleted for $3 \mathrm{~min}$ at $1000 \mathrm{~g}$. Mitotic pellets were washed $5 \mathrm{X}$ in fresh media before replating on to poly-lysine coated glass coverslips. Cells were incubated at $37^{\circ} \mathrm{C}$ in $5 \% \mathrm{CO}_{2}$ and $85 \%$ humidity for $1 \mathrm{~h}$. At $1,2,3,5,7$ and $12 \mathrm{~h}$ post-release, cells were fixed according to the above protocol. Fixed cells were then stained with only DAPI prior to mounting.

Fluorescence microscopy images were acquired using a DeltaVision deconvolution microscope (GE Healthcare Lifesciences) equipped with a CoolSNAP $\mathrm{HQ}^{2}$ camera (Photometrics). Images were collected using either a 60X (numerical aperture $=1.42$; Olympus) oil immersion lens. All images within an experiment were acquired using identical exposure times across all time points. Images were then scored for mitotic index and GFP-Mis18BP1 localization pattern. 
GFP-Mis18BP1 fragment expression and localization analysis

U2OS cells were plated at a density of either 10,400 cells $/ \mathrm{cm}^{2}$ in a 6 -well plate and allowed to attach to the plate and coverslips for DNA transfections. The following day, cells were transfected with $1 \mu \mathrm{g}$ plasmid DNA using Lipofectamine 2000 in OptiMEM according the manufacturer's protocol (Life Technologies). For centromere localization counts of the GFP-tagged Mis18BP1 fragments, cells were transfected with a 10:1 ratio of GFP construct to RFP-H2B plasmid, which served as a transfection marker. The transfection media was removed after $10-12 \mathrm{~h}$ and replaced with fresh growth media. Cells were harvested with 3mM EDTA-PBS for western blot analysis or fixed for immunofluorescence $48 \mathrm{~h}$ post-transfection. Centromeres were highlighted with either CENP-T or CENP-A antibodies. RFP-H2B positive cells were assayed for GFPMis18BP1 fragment localization pattern and score for nuclear, focal centromeric or diffuse centromeric.

\section{Immunoprecipitations}

HeLa GFP-HP1 $\alpha$ cells were left to randomly cycle or were treated with 100 $\mathrm{ng} / \mathrm{mL}$ nocodazole in DMSO for $12-14 \mathrm{~h}$. Randomly cycling cells were harvested with $3 \mathrm{mM}$ EDTA-PBS and mitotics were collected by mitotic shake off. Cells were then pelleted for $5 \mathrm{~min}$ at $1000 \mathrm{~g}$.

For Mis18BP1 immunoprecipitations cell pellets were resuspended in cold lysis buffer (25 mM Tris pH 7.5, $150 \mathrm{mM} \mathrm{KCl,} \mathrm{0.2 \%} \mathrm{NP-40,} 1 \mathrm{mM}$ DTT, $5 \mathrm{mM} \mathrm{NaF}, 50 \mathrm{mM}$ $\beta$-glycerophosphate, $200 \mathrm{mM}$ sodium orthovanadate, $0.5 \mathrm{mM}$ PMSF and $1 \mathrm{X}$ Roche 
protease inhibitor cocktail). Cells were allowed to rest on ice for $10 \mathrm{~min}$ with gentle vortexing. Cell lysates were then sonicated $3 \mathrm{X}$ with a probe sonicator, on ice $(30 \%$ output, $25 \%$ duty cycle for $1 \mathrm{~min}, 2 \mathrm{~min}$ rest), then sonicated for an additional $30 \mathrm{~s} \mathrm{(40 \%}$ output, $25 \%$ duty cycle). Lysates were then centrifuged at $15000 \mathrm{~g}$ for $10 \mathrm{~min}$ at $4^{\circ} \mathrm{C} .1$ $\mu \mathrm{L}$ of anti-Mis18BP1 antibody (1 mg/mL, Bethyl Laboratories, Inc., Cat\# A302-824A) or $1 \mu \mathrm{L}$ rabbit $\operatorname{IgG}(1 \mathrm{mg} / \mathrm{mL})$ as added to lysates and rotated at $4^{\circ} \mathrm{C}$ overnight. Dynabeads were blocked overnight at $4^{\circ} \mathrm{C}$ in $1 \mathrm{mg} / \mathrm{mL}$ BSA. Blocked dynabeads were added to the lysate-antibody mixture for $2 \mathrm{~h}$. Beads were washed $3 \mathrm{X}$ with cold lysis buffer and then resuspended $40 \mu \mathrm{L} 1 \mathrm{X}$ sample buffer (60 mM Tris $\mathrm{HCl} / \mathrm{SDS}$ pH 6.8, 5\% glycerol, 1.67\% SDS, $0.01 \%$ bromophenol blue, $1 \% \beta$-mercaptoethanol).

For GFP immunoprecipitations, cell pellets were resuspended in RIPA buffer (50 $\mathrm{mM}$ Tris $\mathrm{HCl} \mathrm{pH} 8.0,150 \mathrm{mM} \mathrm{NaCl}, 1 \% \mathrm{NP}-40,0.5 \%$ sodium deoxycholate, $0.1 \%$ SDS, $5 \mathrm{mM} \mathrm{NaF}, 50 \mathrm{mM} \beta$-glycerophosphate, $200 \mathrm{mM}$ sodium orthovanadate, $0.5 \mathrm{mM}$ PMSF and $1 \mathrm{X}$ Roche protease inhibitor cocktail). Pellets were left on ice for $15 \mathrm{~min}$ and vortexed every $2 \mathrm{~min}$. Cell lysates were centrifuged $300 \mathrm{~g}$ for $5 \mathrm{~min}$ and the supernatant was treated as in the above IP protocol using anti-GFP (3404 or $1 \mu \mathrm{L}$ rabbit IgG (1 $\mathrm{mg} / \mathrm{mL}$ ) as added to lysates and rotated at $4^{\circ} \mathrm{C}$ overnight. Blocked dynabeads were added to the lysate-antibody mixtures for $2 \mathrm{~h}$ at $4^{\circ} \mathrm{C}$. Beads were washed $3 \mathrm{X}$ with cold RIPA buffer and the resuspended in $40 \mu \mathrm{L} 1 \mathrm{X}$ sample buffer. 
Size exclusion chromatography

HeLa cells stably expressing GFP-Mis18 $\alpha$ (Iain Cheeseman, MIT) were plated and grown to $80 \%$ confluence. Cells were treated with $0.1 \mu \mathrm{g} / \mathrm{mL}$ nocodazole for $14 \mathrm{~h}$ and mitotic cells were collected by washing the plate with media and collecting detached cells. Cell pellets were brought up in ice-cold lysis buffer (3.75 mM Tris pH 7.5, $20 \mathrm{mM}$ $\mathrm{KCl}, 0.5 \mathrm{mM}$ EDTA, 0.5mM DTT, $0.05 \mathrm{mM}$ spermidine, $0.125 \mathrm{mM}$ spermine, $1 \mathrm{mM}$ PMSF, $200 \mu \mathrm{M}$ sodium vanadate, $50 \mathrm{mM} \beta$-glycerophosphate, $0.1 \%$ digitonin, $1 \mathrm{X}$ Roche Protease Inhibitors). Cells were disrupted on ice using a Wheaton glass dounce with a tight pestle. Lysates were centrifuged at $1000 \mathrm{~g}$ for $5 \mathrm{~min}$ and dialyzed into a second buffer $(50 \mathrm{mM}$ Tris- $\mathrm{HCl} \mathrm{pH} 7.5,250 \mathrm{mM} \mathrm{NaCl}, 0.5 \mathrm{mM}$ DTT, 2.5\% glycerol, and $0.05 \%$ NP-40). Lysates were cleared by centrifugation for $10 \mathrm{~min}$ at $1000 \mathrm{~g}$ and separated by FPLC size exclusion chromatography in the dialysis buffer using a Superdex 200 10/300 column (GE Healthcare). Separation was conducted using a Bio-Rad Duo-flow FPLC and $0.5 \mathrm{ml}$ fractions were collected. Fractions were concentrated using TCA precipitation, prior to immunoblotting.

In vitro binding assays

Mis18BP1 was cloned into a modified pMal-c2 vector containing an attR Gateway ${ }^{\circledR}$ cloning cassette inserted into the BamH1 cut site after the N-terminal MBP gene. Mis18BP1 harboring a C-terminal 6X-His tag was cloned into the modified pMalc2 vector using LR Clonase II (Cat\# 11791020, Life Technologies). Entry clones of Mis $18 \alpha$ and Mis $18 \beta$ were generated by recombination from PCR products according to 
the Gateway ${ }^{\circledR}$ cloning protocol (Life Technologies) and were recombined into a HisNusA Gateway® vector (Addgene, pDest-544) using LR clonase II.

Recombinant MBP-tagged Mis18BP1, His-Mis18 $\alpha$ and His-Mis $18 \beta$ proteins were expressed in the Rosetta ${ }^{\mathrm{TM}}$ (DE3) pLysS bacterial strain. Transformed bacteria were grown in $\mathrm{LB}$ media to an $\mathrm{OD}$ of 0.6 at $37^{\circ} \mathrm{C}$, cooled to $18^{\circ} \mathrm{C}$, and protein expression was induced with $0.1 \mathrm{mM} \mathrm{IPTG}$ for $16 \mathrm{~h}$ at $18^{\circ} \mathrm{C}$. Pellets were lysed in $50 \mathrm{mM}$ Tris- $\mathrm{HCl}, 350$ $\mathrm{mM} \mathrm{NaCl}, 0.5 \mathrm{mM} \mathrm{CaCl}_{2}, 10 \%$ glycerol, $0.1 \%$ NP-40, $5 \mathrm{mM} \beta$-mercaptoethanol, LPC, and $1 \mathrm{mM}$ PMSF using a steel Wheaton dounce homogenizer. For His-tagged proteins, 20 $\mathrm{mM}$ imidazole was added to the lysis buffer and $40 \mathrm{mM}$ imidazole was added to the wash buffer. His-tagged proteins were purified using Ni-NTA agarose (Qiagen) eluted with $250 \mathrm{mM}$ imidazole. Mis18BP1 was initially purified using its C-terminal His-tag using the protocol above. It was further purified using a Superdex 200 10/300 column (GEHealthcare). Briefly, elutions were pooled and run over the column in the above buffer (without LPC and PMSF). Peak fractions were pooled and concentrated on Ni-NTA agarose and eluted in the same buffer containing $250 \mathrm{mM}$ imidazole.

In vitro pull-downs were performed in buffer containing $50 \mathrm{mM}$ Tris- $\mathrm{HCl} \mathrm{pH} \mathrm{7.5,}$ $250 \mathrm{mM} \mathrm{NaCl}, 20 \mathrm{mM} \mathrm{MgCl} 2,0.5 \% \mathrm{NP}-40,10 \%$ glycerol, and $5 \mathrm{mM} \beta$ mercaptoethanol. Recombinant proteins combined at 1:1 molar ratio and incubated for 3 $\mathrm{h}$ at room temperature. Affinity matrices were pre-incubated for $1 \mathrm{~h}$ at room temperature in the same buffer supplemented with $0.2 \mathrm{mg} / \mathrm{mL}$ BSA. The blocked matrices were added to the pre-formed complexes and incubated for $40 \mathrm{~min}$ at room temperature. Wash buffer for Ni-NTA pull-downs, which was supplemented with $40 \mathrm{mM}$ imidazole. The matrices 
were resuspended in sample buffer boiled. Immunoblots were performed using antibodies against the 6X-His tags (sc-803, Santa Cruz Biotechnologies) and MBP tag (New England Biolabs).

siRNA depletions

Simple siRNA depletions of Mis 18 proteins in cells that stably expressed GFPMis 18BP1 or GFP-Mis18 $\alpha$ were accomplished by plating cells at a density of 10,400 cells $/ \mathrm{cm}^{2}$ in a 6-well format and letting the cells attach to the plate and coverslips. The following day, cells were treated with $50 \mathrm{nM}$ Silencer ${ }^{\circledR}$ Select siRNA (HP1 $\alpha$ (CBX-5), ID\#: s23884, Cat\#: 4392420, Ambion; GAPDH Positive Control siRNA, Cat\# 4390850, Ambion) using RNAiMax in Opti-MEM. After $12-24 \mathrm{~h}, 1 \mathrm{~mL}$ of full growth media was added to supplement the reduced serum media. Cells were then harvested $48 \mathrm{~h}$ postsiRNA transfection using 3mM EDTA-PBS or fixed and stained using the previously stated protocol.

The degree of siRNA depletion was determined by immunoblot and protein depletion was estimated by a dilution series of control lysates. Cell lysates that corresponded to $1 \times 10^{5}$ cells were separated on $10 \%$ polyacrylamide gels and blotted to nitrocellulose using standard approaches. The nitrocellulose was blocked for $1 \mathrm{~h}$ in blocking buffer: $5 \%$ milk in TBST ( $137 \mathrm{mM} \mathrm{NaCl}, 2.7 \mathrm{mM} \mathrm{KCl}, 25 \mathrm{mM}$ Tris Base, $0.1 \%$ Tween 20). Primary antibodies were incubated overnight in blocking buffer at $4^{\circ} \mathrm{C}$ [Mis18BP1 (Bethyl Laboratories, Inc., Cat\# A302-824A), and HP1 $\alpha$ (CBX-5, A300877A, Bethyl Laboratories, Inc.). HRP conjugated secondary antibodies were incubated 
in blocking buffer at $40 \mathrm{ng} / \mathrm{mL}$ for $1 \mathrm{~h}$ at room temperature (Goat-anti-rabbit, Cat\# 1110.35-003, Jackson ImmunoResearch Laboratories, Inc.). HRP was detected using the West Pico or West Femto ECL Regent (Pierce) chemiluminescent substrate.

\section{Additional interactions with the CCAN:}

\section{Tissue culture}

HeLa GFP-Mis18 $\alpha$ cells lines were cultured in DMEM High Glucose (Life Technologies) supplemented with 10\% heat inactivated FBS (Optima, Atlanta Biologicals) and 1X Penicillin/Streptomycin (Life Technologies). U2OS derived cell lines were cultured in DMEM High Glucose GlutaMAX ${ }^{\mathrm{TM}}$ (Life Technologies) supplemented with 10\% FBS and 1X Penicillin/Streptomycin. Cells were incubated at $37^{\circ} \mathrm{C}$ in $5 \% \mathrm{CO}_{2}$ and $85 \%$ humidity.

siRNA depletion and western blot

Simple siRNA depletions of Mis 18 proteins in U2OS cells and cells that stably expressed GFP-Mis18 $\alpha$ were accomplished by plating cells at a density of 10,400 cells $/ \mathrm{cm}^{2}$ in a 6-well format and letting the cells attach to the plate and coverslips. The following day, cells were treated with 20 nM Silencer® Select siRNA (Mis18BP1, 5'GGAUAUCCAAAUUAUCUCAtt-3'; Mis18 $\alpha$, 5'-GAAGAUGUCUUGAAAGCAUtt3'; HJURP, 5'-CAAGUAUGGAAGUUCGAUAtt-3'; Negative Control \#2, Cat\# 4390846, Ambion) using RNAiMax in Opti-MEM. After $12-24$ h, 1 mL of full growth media was added to supplement the reduced serum media. Cells were then harvested $48 \mathrm{~h}$ 
post-siRNA transfection using 3mM EDTA-PBS or fixed and stained using the protocol below.

The degree of siRNA depletion was determined by immunoblot and protein depletion was estimated by a dilution series of untreated lysates. Cell lysates that corresponded to $1 \times 10^{5}$ cells were separated on $10 \%$ polyacrylamide gels and blotted to nitrocellulose using standard approaches. The nitrocellulose was blocked for $1 \mathrm{~h}$ in blocking buffer: 5\% milk in TBST (137 mM NaCl, $2.7 \mathrm{mM} \mathrm{KCl,} 25 \mathrm{mM}$ Tris Base, 0.1\% Tween 20). Primary antibodies were incubated overnight in blocking buffer at $4^{\circ} \mathrm{C}$ [Mis18BP1 (Bethyl Laboratories, Inc., Cat\# A302-824A); Mis18ß (BL10295, Bethyl Laboratories, Inc.); and HJURP (antibody 3399)]. HRP conjugated secondary antibodies were incubated in blocking buffer at $40 \mathrm{ng} / \mathrm{mL}$ for $1 \mathrm{~h}$ at room temperature (Goat-antirabbit, Cat\# 111-0.35-003, Jackson ImmunoResearch Laboratories, Inc.). HRP was detected using the West Pico ECL Regent (Pierce) chemiluminescent substrate.

\section{Immunofluorescence preparation and staining protocol}

U2OS and HeLa GFP-Mis18 $\alpha$ cells grown on poly-lysine coverslips were fixed for $10 \mathrm{~min}$ with $37^{\circ} \mathrm{C} 4 \%$ formaldehyde in PBS $(137 \mathrm{mM} \mathrm{NaCl}, 2.7 \mathrm{mM} \mathrm{KCl}, 5.4 \mathrm{mM}$ $\mathrm{Na}_{2} \mathrm{HPO}_{4}, 1.8 \mathrm{mM} \mathrm{KH}_{2} \mathrm{PO}_{4}$ ) and then quenched for 5 min with $100 \mathrm{mM}$ Tris $\mathrm{pH}$ 7.5. Coverslips were blocked for $1 \mathrm{~h}$ in blocking buffer: $2 \%$ heat inactivated FBS, $2 \%$ BSA, and $0.1 \%$ Triton-X100 in PBS. Primary antibodies were incubated for $1 \mathrm{~h}$ in blocking buffer: anti-CENP-A mouse monoclonal antibody (ab13939, Abcam) at $1 \mu \mathrm{g} / \mathrm{mL}$, antiCENP-C mouse monoclonal antibody (custom) at 1:3000, and CENP-H and CENP-I (gift 
from the P. T. Stukenberg lab) at 1:1000. Coverslips were washed three times for 5 min with $0.1 \%$ Triton-X100 in PBS. Secondary antibodies were diluted in blocking buffer and were incubated with the coverslips for $1 \mathrm{~h}$ (Alexa-647 conjugated goat-anti-mouse at $0.375 \mu \mathrm{g} / \mathrm{mL}$, Cy3 conjugated goat-anti-mouse at $0.75 \mu \mathrm{g} / \mathrm{mL}$; Jackson ImmunoResearch Laboratories, Inc.). Cells were washed as above and then incubated in PBS containing 0.2 $\mu \mathrm{g} / \mathrm{mL}$ of DAPI for 5 min. Coverslips were rinsed with PBS prior to mounting onto glass slides with Prolong Gold Antifade Reagent (Life Technologies).

\section{Centromere intensity analysis}

CENP-I, CENP-H and CENP-A intensity at centromeres was measured from nondeconvolved maximum projections, using ImageJ [175]. The integrated intensity of at least five random centromeres in each nucleus was measured across $\geq 35$ cells. The $95 \%$ confidence intervals were calculated for each measurement population. Mann-Whitney statistical analysis was used to determine statistical differences between the medians of the centromere intensities for each condition at a confidence value of $95 \%$.

\section{Salt extraction and intensity analsys}

HeLa GFP-Mis18 $\alpha$ were grown on poly-lysine coated coverslips until 75\% confluence. To perform the salt wash, growth media was removed from the cells and replaced with PBS alone (low salt) or PBS supplemented with $500 \mathrm{mM} \mathrm{NaCl}$ (high salt) that had been pre-warmed to $37^{\circ} \mathrm{C}$. Cells were fixed either prior to the salt wash (PostFix) or after the salt wash (Pre-Fix) according to the standard fixation techniques 
described above. Fixed cells were then stained for endogenous CENP-C (Cy5 as the secondary fluorophore) and images according to the standard techniques above.

To analyze the changes in intensity between the low and high salt wash conditions, maximum projections of deconvolved z-stacks were analyze by ImageJ software [175]. Entire nuclei were bounded by DAPI staining and the maximum intensity of GFP-Mis $18 \alpha$ and CENP-C (Cy5) in each nucleus was recorded along with its GFPMis $18 \alpha$ localization status. The maximum intensity measurements of each condition were averaged within each replicate. This average intensity was set to 1 (or 100\%) in the low salt condition. Statistical significance was determined by the student's t-test.

\section{Possible histone binding capabilities of Mis18BP1:}

\section{Subcellular fractionation}

Cells were harvested with $3 \mathrm{mM}$ EDTA-PBS, pelleted and washed in PBS. Cell pellets were resuspended in Buffer A (10 mM HEPES pH 7.9, $1.5 \mathrm{mM} \mathrm{MgCl}_{2}, 10 \mathrm{mM}$ $\mathrm{KCl}, 0.5 \mathrm{mM} \mathrm{DTT}$, and $0.2 \mathrm{mM}$ PMSF) and incubated on $10 \mathrm{~min}$ (whole cell). Cell resuspensions were lysed with a tight Wheaton glass dounce and lysates were centrifuged $30 \mathrm{~s}$ at $14000 \mathrm{~g}$ and the supernatant was the cytoplasmic faction. Pellet was resuspended in Buffer B (20 mM HEPES pH 7.9, 25\% glycerol, $420 \mathrm{mM} \mathrm{NaCl}, 1.5 \mathrm{mM} \mathrm{MgCl}_{2}, 0.2$ mM EDTA, 0.5 DTT, and 0.2 PMSF) and was the whole nuclear fraction. Add triton X100 to a final concentration of $1 \%$ to the resuspended nuclei and incubate on ice for 10 min. Centrifuge $10 \mathrm{~min}$ at $14000 \mathrm{~g}$. Supernatant was the nucleoplasm fraction and the final pellet was the chromatin fraction. 
In order to analyze fractions by western blot, glycerol concentrations were equalized across fractions before loading into $10 \%$ polyacrylamide gels and blotted to nitrocellulose using standard approaches. The nitrocellulose was blocked for $1 \mathrm{~h}$ in blocking buffer: 5\% milk in TBST. Primary antibodies were incubated overnight in blocking buffer at $4^{\circ} \mathrm{C}$ [Mis18BP1 (Bethyl Laboratories, Inc., Cat\# A302-824A); Nucleophosmin]. HRP conjugated secondary antibodies were incubated in blocking buffer at $40 \mathrm{ng} / \mathrm{mL}$ for $1 \mathrm{~h}$ at room temperature (Goat-anti-rabbit, Cat\# 111-0.35-003, Jackson ImmunoResearch Laboratories, Inc.). HRP was detected using the West Pico ECL Regent chemiluminescent substrate (Pierce).

\section{GST fusion protein purification}

Rosetta bacteria transformed with GST-histone tails plasmids were grown overnight. One liter of LB was inoculated with the overnight culture and grown to on OD of 0.6 at $37^{\circ} \mathrm{C}$. Cultures were then induced with $1 \mathrm{mM}$ IPTG for $3 \mathrm{~h}$ at $37^{\circ} \mathrm{C}$. Induced cultures were centrifuged at $8000 \mathrm{~g}$ in a SLA-3000 rotor for $10 \mathrm{~min}$. Pellets were resuspended in $\mathrm{LB}$ and transferred to $50 \mathrm{~mL}$ conical tubes. Resuspended pellets were centrifuged again at $4000 \mathrm{~g}$ for $10 \mathrm{~min}$. Supernatant was removed and the pellets were flash frozen for long-term storage.

Frozen pellets were thawed on ice in lysis buffer $(50 \mathrm{mM}$ Tris $\mathrm{pH} 7.5,150 \mathrm{mM}$ $\mathrm{NaCl}, 10 \mathrm{mM}$ reduced glutathione, $10 \mathrm{mM} \beta$-glycerophosphate, $1.5 \mathrm{mM} \mathrm{MgCl} 2,1 \mathrm{mM}$ DTT). Lysozyme was added to a concentration of $1 \mathrm{mg} / \mathrm{mL}$ and cell resuspensions were incubated on ice for $30 \mathrm{~min}$, then they were processed with a metal dounce until all 
viscosity was lost. Lysates were centrifuged at $10000 \mathrm{~g}$ for $10 \mathrm{~min}$ at $4^{\circ} \mathrm{C}$. Glutathione sepharose was added to the supernatant and rocked for $1.5 \mathrm{~h}$ at $4^{\circ} \mathrm{C}$. The sepharose was centrifuge $300 \mathrm{rpm}$ for $3 \mathrm{~min}$. The pellet was resuspended in lysis buffer and loaded into a column. The sepharose was washed $3 \mathrm{X}$ with lysis buffer. Then the GST-histone tail protein was eluted $5 \mathrm{X}$ with elution buffer $(50 \mathrm{mM}$ Tris $\mathrm{pH} 7.5,150 \mathrm{mM} \mathrm{NaCl}, 10 \mathrm{mM}$ reduced glutathione, $10 \mathrm{mM} \beta$-glycerophosphate, $1.5 \mathrm{mM} \mathrm{MgCl}_{2}, 1 \mathrm{mM} \mathrm{DTT).}$

\section{His fusion protein purification}

Rosetta bacteria transformed with His-Mis $18 \mathrm{BP} 1^{\text {SANT }}$ plasmids were grown overnight. One liter of LB was inoculated with the overnight culture and grown to on OD of 0.6 at $37^{\circ} \mathrm{C}$. Cultures were then induced with $1 \mathrm{mM}$ IPTG for $3 \mathrm{~h}$ at $37^{\circ} \mathrm{C}$. Induced cultures were centrifuged at $8000 \mathrm{~g}$ in a SLA-3000 rotor for $10 \mathrm{~min}$. Pellets were resuspended in LB and transferred to $50 \mathrm{~mL}$ conical tubes. Resuspended pellets were centrifuged again at $4000 \mathrm{~g}$ for $10 \mathrm{~min}$. Supernatant was removed and the pellets were flash frozen for long-term storage.

Frozen pellets were thawed on ice in lysis buffer $(50 \mathrm{mM}$ phosphate buffer $\mathrm{pH}$ 7.5, $300 \mathrm{mM} \mathrm{NaCl}, 10 \mathrm{mM}$ imidizole, $10 \mathrm{mM} \beta$-glycerophosphate, $1.5 \mathrm{mM} \mathrm{MgCl} 2,1$ mM DTT, 0.5 PMSF, 1X LPC). Lysozyme was added to a concentration of $1 \mathrm{mg} / \mathrm{mL}$ and cell resuspensions were incubated on ice for $30 \mathrm{~min}$, then they were processed with a metal dounce until all viscosity was lost. Lysates were centrifuged at $10000 \mathrm{~g}$ for $10 \mathrm{~min}$ at $4^{\circ} \mathrm{C}$. Nickel-NTA agarose was added to the supernatant and rocked for $1.5 \mathrm{~h}$ at $4{ }^{\circ} \mathrm{C}$. The agarose was centrifuge $300 \mathrm{rpm}$ for $3 \mathrm{~min}$. The pellet was resuspended in lysis buffer 
and loaded into a column. The sepharose was washed $3 \mathrm{X}$ with lysis buffer. Then the GST-histone tail protein was eluted $5 \mathrm{X}$ with elution buffer $(50 \mathrm{mM}$ phosphate buffer $\mathrm{pH}$ 7.5, $150 \mathrm{mM} \mathrm{NaCl}, 250 \mathrm{mM}$ imidizole, $10 \mathrm{mM} \beta$-glycerophosphate, $1.5 \mathrm{mM} \mathrm{MgCl} 2,1$ mM DTT).

Purified proteins were run out on a $12 \%$ SDS-PAGE gel. Separated proteins were then stained with coomassie $(0.2 \%$ R-250 coomassie, $50 \%$ methanol, $10 \%$ acetic acid) for $1 \mathrm{~h}$. The gels were incubated in destain buffer (73\% water, $20 \%$ ethanol $(95 \%)$, and $7 \%$ acetic acid) overnight. Destained gels were dried in cellophane prior to imaging.

\section{GST pull down protocols}

Protocol 1: $10 \mu \mathrm{g}$ of each GST-histone tail was incubated with HisMis $18 \mathrm{BP} 1^{\text {SANT }}$ in incubation buffer $(20 \mathrm{mM}$ Tris $\mathrm{pH} 8.0,50 \mathrm{mM} \mathrm{NaCl}, 1 \mathrm{mM}$ EDTA, 1 mM DTT, $0.2 \%$ triton X-100, 10\% glycerol). The constructs were rotated for $3 \mathrm{~h}$ at $4{ }^{\circ} \mathrm{C}$. After incubation, $10 \mu \mathrm{L}$ of glutathione sepharose was added to the GST and His-tagged constructs and rotated for $30 \mathrm{~min}$ at $4^{\circ} \mathrm{C}$. The sepharose was washed for $3 \mathrm{X}$ with incubation buffer and then pelleted at $500 \mathrm{~g}$ for $3 \mathrm{~min}$. The supernatant was removed and was the unbound fraction. Then the sepharose was resuspended in $1 \mathrm{X}$ sample buffer to generate the bound fraction.

Protocol 2: $10 \mu \mathrm{g}$ of each GST-histone tail was incubated with glutathione sepharose for $30 \mathrm{~min}$ at $4^{\circ} \mathrm{C}$ incubation buffer. The sepharose was washed for $3 \mathrm{X}$ with buffer. $4 \mu \mathrm{g}$ of His-Mis18BP1 ${ }^{\text {SANT }}$ was added to the sepharose bound GST-histone tails and incubated for $3 \mathrm{~h}$ at $4^{\circ} \mathrm{C}$. The sepharose was washed for $3 \mathrm{X}$ with buffer and then 
pelleted at $500 \mathrm{~g}$ for $3 \mathrm{~min}$. The supernatant was removed and was the unbound fraction. Then the sepharose was resuspended in 1X sample buffer to generate the bound fraction. Bound and unbound fractions from both protocols were run out on 12\% SDS-PAGE to separate the GST-histone tails and His-Mis $18 \mathrm{BP}^{\mathrm{SANT}}{ }^{\mathrm{S}}$ proteins. After separation on the gel, the presence of His-Mis $18 \mathrm{BP} 1^{\text {SANT }}$ in the bound or unbound fraction was determined by western blotting (anti-His, clone H-15, sc-803, Santa Cruz Biotechnology; Goat-antirabbit, Cat\# 111-0.35-003, Jackson ImmunoResearch Laboratories, Inc.) and coomassie staining using the previously described protocols. 


\section{References}

1. Torres, EM, Williams, BR, and Amon, A. Aneuploidy: cells losing their balance. Genetics. (2008) 179(2): p. 737-46.

2. McClintock, B. The Stability of Broken Ends of Chromosomes in Zea Mays. Genetics. (1941) 26(2): p. 234-82.

3. Colnaghi, R, Carpenter, G, Volker, M, and O'Driscoll, M. The consequences of structural genomic alterations in humans: Genomic Disorders, genomic instability and cancer. Semin Cell Dev Biol. (2011) 22(8): p. 875-85.

4. Stoler, S, Keith, KC, Curnick, KE, and Fitzgerald-Hayes, M. A mutation in CSE4, an essential gene encoding a novel chromatin-associated protein in yeast, causes chromosome nondisjunction and cell cycle arrest at mitosis. Genes Dev. (1995) 9(5): p. 573-86.

5. Howman, EV, Fowler, KJ, Newson, AJ, Redward, S, MacDonald, AC, Kalitsis, $\mathrm{P}$, and Choo, KH. Early disruption of centromeric chromatin organization in centromere protein A (Cenpa) null mice. Proc Natl Acad Sci U S A. (2000) 97(3): p. 1148-53.

6. Cottarel, G, Shero, JH, Hieter, P, and Hegemann, JH. A 125-base-pair CEN6 DNA fragment is sufficient for complete meiotic and mitotic centromere functions in Saccharomyces cerevisiae. Mol Cell Biol. (1989) 9(8): p. 3342-9.

7. Clarke, L and Carbon, J. Isolation of a yeast centromere and construction of functional small circular chromosomes. Nature. (1980) 287(5782): p. 504-9.

8. Maddox, PS, Oegema, K, Desai, A, and Cheeseman, IM. "Holo"er than thou: chromosome segregation and kinetochore function in C. elegans. Chromosome Res. (2004) 12(6): p. 641-53.

9. Sanchez-Pulido, L, Pidoux, AL, Ponting, CP, and Allshire, RC. Common ancestry of the CENP-A chaperones Scm3 and HJURP. Cell. (2009) 137(7): p. 1173-4.

10. Enukashvily, NI and Ponomartsev, NV. Mammalian satellite DNA: a speaking dumb. Adv Protein Chem Struct Biol. (2013) 90: p. 31-65.

11. Marshall, OJ, Chueh, AC, Wong, LH, and Choo, KH. Neocentromeres: new insights into centromere structure, disease development, and karyotype evolution. Am J Hum Genet. (2008) 82(2): p. 261-82.

12. Bassett, EA, Wood, S, Salimian, KJ, Ajith, S, Foltz, DR, and Black, BE. Epigenetic centromere specification directs aurora $B$ accumulation but is insufficient to efficiently correct mitotic errors. J Cell Biol. (2010) 190(2): p. 177-85. 
13. Lo, AW, Craig, JM, Saffery, R, Kalitsis, P, Irvine, DV, Earle, E, Magliano, DJ, and Choo, KH. A $330 \mathrm{~kb}$ CENP-A binding domain and altered replication timing at a human neocentromere. EMBO J. (2001) 20(8): p. 2087-96.

14. Warburton, PE, Cooke, CA, Bourassa, S, Vafa, O, Sullivan, BA, Stetten, G, Gimelli, G, Warburton, D, Tyler-Smith, C, Sullivan, KF, Poirier, GG, and Earnshaw, WC. Immunolocalization of CENP-A suggests a distinct nucleosome structure at the inner kinetochore plate of active centromeres. Curr Biol. (1997) 7(11): p. 901-4.

15. Voullaire, LE, Slater, HR, Petrovic, V, and Choo, KH. A functional marker centromere with no detectable alpha-satellite, satellite III, or CENP-B protein: activation of a latent centromere? Am J Hum Genet. (1993) 52(6): p. 1153-63.

16. Ishii, K, Ogiyama, Y, Chikashige, Y, Soejima, S, Masuda, F, Kakuma, T, Hiraoka, Y, and Takahashi, K. Heterochromatin integrity affects chromosome reorganization after centromere dysfunction. Science. (2008) 321(5892): p. 1088-91.

17. Williams, BC, Murphy, TD, Goldberg, ML, and Karpen, GH. Neocentromere activity of structurally acentric mini-chromosomes in Drosophila. Nat Genet. (1998) 18(1): p. 30-7.

18. Barnhart, MC, Kuich, PH, Stellfox, ME, Ward, JA, Bassett, EA, Black, BE, and Foltz, DR. HJURP is a CENP-A chromatin assembly factor sufficient to form a functional de novo kinetochore. J Cell Biol. (2011) 194(2): p. 229-43.

19. Mendiburo, MJ, Padeken, J, Fulop, S, Schepers, A, and Heun, P. Drosophila CENH3 is sufficient for centromere formation. Science. (2011) 334(6056): p. 686-90.

20. Obuse, C, Yang, H, Nozaki, N, Goto, S, Okazaki, T, and Yoda, K. Proteomics analysis of the centromere complex from HeLa interphase cells: UV-damaged DNA binding protein 1 (DDB-1) is a component of the CEN-complex, while BMI-1 is transiently co-localized with the centromeric region in interphase. Genes Cells. (2004) 9(2): p. 105-20.

21. Foltz, DR, Jansen, LE, Black, BE, Bailey, AO, Yates, JR, 3rd, and Cleveland, DW. The human CENP-A centromeric nucleosome-associated complex. Nat Cell Biol. (2006) 8(5): p. 458-69.

22. Okada, M, Cheeseman, IM, Hori, T, Okawa, K, McLeod, IX, Yates, JR, 3rd, Desai, A, and Fukagawa, T. The CENP-H-I complex is required for the efficient incorporation of newly synthesized CENP-A into centromeres. Nat Cell Biol. (2006) 8(5): p. 446-57. 
23. Mizuguchi, G, Xiao, H, Wisniewski, J, Smith, MM, and Wu, C. Nonhistone Scm3 and histones CenH3-H4 assemble the core of centromere-specific nucleosomes. Cell. (2007) 129(6): p. 1153-64.

24. Dimitriadis, EK, Weber, C, Gill, RK, Diekmann, S, and Dalal, Y. Tetrameric organization of vertebrate centromeric nucleosomes. Proc Natl Acad Sci U S A. (2010) 107(47): p. 20317-22.

25. Dalal, Y, Wang, H, Lindsay, S, and Henikoff, S. Tetrameric structure of centromeric nucleosomes in interphase Drosophila cells. PLoS Biol. (2007) 5(8): p. e218.

26. Tachiwana, H, Kagawa, W, Shiga, T, Osakabe, A, Miya, Y, Saito, K, Hayashi-Takanaka, Y, Oda, T, Sato, M, Park, SY, Kimura, H, and Kurumizaka, H. Crystal structure of the human centromeric nucleosome containing CENP-A. Nature. (2011) 476(7359): p. 232-5.

27. Zhang, W, Colmenares, SU, and Karpen, GH. Assembly of Drosophila Centromeric Nucleosomes Requires CID Dimerization. Mol Cell. (2012) 45(2): p. 263-9.

28. Bassett, EA, DeNizio, J, Barnhart-Dailey, MC, Panchenko, T, Sekulic, N, Rogers, DJ, Foltz, DR, and Black, BE. HJURP uses distinct CENP-A surfaces to recognize and to stabilize CENP-A/histone $\mathrm{H} 4$ for centromere assembly. Dev Cell. (2012) 22(4): p. 749-62.

29. Black, BE, Brock, MA, Bedard, S, Woods, VL, Jr., and Cleveland, DW. An epigenetic mark generated by the incorporation of CENP-A into centromeric nucleosomes. Proc Natl Acad Sci U S A. (2007) 104(12): p. 5008-13.

30. Black, BE, Foltz, DR, Chakravarthy, S, Luger, K, Woods, VL, Jr., and Cleveland, DW. Structural determinants for generating centromeric chromatin. Nature. (2004) 430(6999): p. 578-82.

31. Panchenko, T, Sorensen, TC, Woodcock, CL, Kan, ZY, Wood, S, Resch, MG, Luger, K, Englander, SW, Hansen, JC, and Black, BE. Replacement of histone $\mathrm{H} 3$ with CENP-A directs global nucleosome array condensation and loosening of nucleosome superhelical termini. Proc Natl Acad Sci U $S$ A. (2011) 108(40): p. 16588-93.

32. Conde e Silva, N, Black, BE, Sivolob, A, Filipski, J, Cleveland, DW, and Prunell, A. CENP-A-containing nucleosomes: easier disassembly versus exclusive centromeric localization. J Mol Biol. (2007) 370(3): p. 555-73.

33. Sekulic, N, Bassett, EA, Rogers, DJ, and Black, BE. The structure of (CENP-A-H4)(2) reveals physical features that mark centromeres. Nature. (2010) 467(7313): p. 347-51. 
34. Zeitlin, SG, Barber, CM, Allis, CD, and Sullivan, KF. Differential regulation of CENP-A and histone H3 phosphorylation in G2/M. J Cell Sci. (2001) 114(Pt 4): p. 653-61.

35. Zeitlin, SG, Shelby, RD, and Sullivan, KF. CENP-A is phosphorylated by Aurora B kinase and plays an unexpected role in completion of cytokinesis. J Cell Biol. (2001) 155(7): p. 1147-57.

36. Kunitoku, N, Sasayama, T, Marumoto, T, Zhang, D, Honda, S, Kobayashi, O, Hatakeyama, K, Ushio, Y, Saya, H, and Hirota, T. CENP-A phosphorylation by Aurora-A in prophase is required for enrichment of Aurora-B at inner centromeres and for kinetochore function. Dev Cell. (2003) 5(6): p. 853-64.

37. Goutte-Gattat, D, Shuaib, M, Ouararhni, K, Gautier, T, Skoufias, DA, Hamiche, A, and Dimitrov, S. Phosphorylation of the CENP-A aminoterminus in mitotic centromeric chromatin is required for kinetochore function. Proc Natl Acad Sci U S A. (2013) 110(21): p. 8579-84.

38. Petkowski, JJ, Schaner Tooley, CE, Anderson, LC, Shumilin, IA, Balsbaugh, JL, Shabanowitz, J, Hunt, DF, Minor, W, and Macara, IG. Substrate specificity of mammalian N-terminal alpha-amino methyltransferase NRMT. Biochemistry. (2012) 51(30): p. 5942-50.

39. Tooley, CE, Petkowski, JJ, Muratore-Schroeder, TL, Balsbaugh, JL, Shabanowitz, J, Sabat, M, Minor, W, Hunt, DF, and Macara, IG. NRMT is an alpha-N-methyltransferase that methylates RCC1 and retinoblastoma protein. Nature. (2010) 466(7310): p. 1125-8.

40. Bailey, AO, Panchenko, T, Sathyan, KM, Petkowski, JJ, Pai, PJ, Bai, DL, Russell, DH, Macara, IG, Shabanowitz, J, Hunt, DF, Black, BE, and Foltz, DR. Posttranslational modification of CENP-A influences the conformation of centromeric chromatin. Proc Natl Acad Sci U S A. (2013) 110(29): p. 11827-32.

41. Blower, MD, Sullivan, BA, and Karpen, GH. Conserved organization of centromeric chromatin in flies and humans. Dev Cell. (2002) 2(3): p. 319-30.

42. Lam, AL, Boivin, CD, Bonney, CF, Rudd, MK, and Sullivan, BA. Human centromeric chromatin is a dynamic chromosomal domain that can spread over noncentromeric DNA. Proc Natl Acad Sci U S A. (2006) 103(11): p. 4186-91.

43. Sullivan, BA and Karpen, GH. Centromeric chromatin exhibits a histone modification pattern that is distinct from both euchromatin and heterochromatin. Nat Struct Mol Biol. (2004) 11(11): p. 1076-83.

44. Bergmann, JH, Rodriguez, MG, Martins, NM, Kimura, H, Kelly, DA, Masumoto, H, Larionov, V, Jansen, LE, and Earnshaw, WC. Epigenetic 
engineering shows H3K4me2 is required for HJURP targeting and CENP-A assembly on a synthetic human kinetochore. EMBO J. (2011) 30(2): p. 328-40.

45. Cardinale, S, Bergmann, JH, Kelly, D, Nakano, M, Valdivia, MM, Kimura, H, Masumoto, H, Larionov, V, and Earnshaw, WC. Hierarchical inactivation of a synthetic human kinetochore by a chromatin modifier. Mol Biol Cell. (2009) 20(19): p. 4194-204.

46. Nakano, M, Cardinale, S, Noskov, VN, Gassmann, R, Vagnarelli, P, Kandels-Lewis, S, Larionov, V, Earnshaw, WC, and Masumoto, H. Inactivation of a human kinetochore by specific targeting of chromatin modifiers. Dev Cell. (2008) 14(4): p. 507-22.

47. Ohzeki, J, Bergmann, JH, Kouprina, N, Noskov, VN, Nakano, M, Kimura, $\mathrm{H}$, Earnshaw, WC, Larionov, V, and Masumoto, H. Breaking the HAC Barrier: histone H3K9 acetyl/methyl balance regulates CENP-A assembly. EMBO J. (2012) 31(10): p. 2391-402.

48. Nishino, T, Takeuchi, K, Gascoigne, KE, Suzuki, A, Hori, T, Oyama, T, Morikawa, K, Cheeseman, IM, and Fukagawa, T. CENP-T-W-S-X Forms a Unique Centromeric Chromatin Structure with a Histone-like Fold. Cell. (2012) 148(3): p. 487-501.

49. Marino-Ramirez, L, Levine, KM, Morales, M, Zhang, S, Moreland, RT, Baxevanis, AD, and Landsman, D. The Histone Database: an integrated resource for histones and histone fold-containing proteins. Database (Oxford). (2011) 2011: p. bar048.

50. Hori, T, Amano, M, Suzuki, A, Backer, CB, Welburn, JP, Dong, Y, McEwen, BF, Shang, WH, Suzuki, E, Okawa, K, Cheeseman, IM, and Fukagawa, T. CCAN makes multiple contacts with centromeric DNA to provide distinct pathways to the outer kinetochore. Cell. (2008) 135(6): p. 1039-52.

51. Prendergast, L, van Vuuren, C, Kaczmarczyk, A, Doering, V, Hellwig, D, Quinn, N, Hoischen, C, Diekmann, S, and Sullivan, KF. Premitotic assembly of human CENPs -T and -W switches centromeric chromatin to a mitotic state. PLoS Biol. (2011) 9(6): p. e1001082.

52. Ribeiro, SA, Vagnarelli, P, Dong, Y, Hori, T, McEwen, BF, Fukagawa, T, Flors, C, and Earnshaw, WC. A super-resolution map of the vertebrate kinetochore. Proc Natl Acad Sci U S A. (2010) 107(23): p. 10484-9.

53. Marshall, OJ, Marshall, AT, and Choo, KH. Three-dimensional localization of CENP-A suggests a complex higher order structure of centromeric chromatin. J Cell Biol. (2008) 183(7): p. 1193-202. 
54. Zheng, C, Lu, X, Hansen, JC, and Hayes, JJ. Salt-dependent intra- and internucleosomal interactions of the $\mathrm{H} 3$ tail domain in a model oligonucleosomal array. J Biol Chem. (2005) 280(39): p. 33552-7.

55. Peters, AH, Kubicek, S, Mechtler, K, O'Sullivan, RJ, Derijck, AA, PerezBurgos, L, Kohlmaier, A, Opravil, S, Tachibana, M, Shinkai, Y, Martens, $\mathrm{JH}$, and Jenuwein, T. Partitioning and plasticity of repressive histone methylation states in mammalian chromatin. Mol Cell. (2003) 12(6): p. 1577-89.

56. Rice, JC, Briggs, SD, Ueberheide, B, Barber, CM, Shabanowitz, J, Hunt, DF, Shinkai, Y, and Allis, CD. Histone methyltransferases direct different degrees of methylation to define distinct chromatin domains. Mol Cell. (2003) 12(6): p. 1591-8.

57. Folco, HD, Pidoux, AL, Urano, T, and Allshire, RC. Heterochromatin and RNAi are required to establish CENP-A chromatin at centromeres. Science. (2008) 319(5859): p. 94-7.

58. Grewal, SI. RNAi-dependent formation of heterochromatin and its diverse functions. Curr Opin Genet Dev. (2010) 20(2): p. 134-41.

59. Kagansky, A, Folco, HD, Almeida, R, Pidoux, AL, Boukaba, A, Simmer, F, Urano, T, Hamilton, GL, and Allshire, RC. Synthetic heterochromatin bypasses RNAi and centromeric repeats to establish functional centromeres. Science. (2009) 324(5935): p. 1716-9.

60. Olszak, AM, van Essen, D, Pereira, AJ, Diehl, S, Manke, T, Maiato, H, Saccani, S, and Heun, P. Heterochromatin boundaries are hotspots for de novo kinetochore formation. Nat Cell Biol. (2011) 13(7): p. 799-808.

61. Alonso, A, Hasson, D, Cheung, F, and Warburton, PE. A paucity of heterochromatin at functional human neocentromeres. Epigenetics Chromatin. (2010) 3(1): p. 6.

62. Guse, A, Carroll, CW, Moree, B, Fuller, CJ, and Straight, AF. In vitro centromere and kinetochore assembly on defined chromatin templates. Nature. (2011) 477(7364): p. 354-8.

63. Carroll, CW, Milks, KJ, and Straight, AF. Dual recognition of CENP-A nucleosomes is required for centromere assembly. J Cell Biol. (2010) 189(7): p. 1143-55.

64. Black, BE, Jansen, LE, Maddox, PS, Foltz, DR, Desai, AB, Shah, JV, and Cleveland, DW. Centromere identity maintained by nucleosomes assembled with histone H3 containing the CENP-A targeting domain. Mol Cell. (2007) 25(2): p. 309-22.

65. Carroll, CW, Silva, MC, Godek, KM, Jansen, LE, and Straight, AF. Centromere assembly requires the direct recognition of CENP-A nucleosomes by CENP-N. Nat Cell Biol. (2009) 11(7): p. 896-902. 
66. Dunleavy, EM, Roche, D, Tagami, H, Lacoste, N, Ray-Gallet, D, Nakamura, Y, Daigo, Y, Nakatani, Y, and Almouzni-Pettinotti, G. HJURP is a cell-cycle-dependent maintenance and deposition factor of CENP-A at centromeres. Cell. (2009) 137(3): p. 485-97.

67. Milks, KJ, Moree, B, and Straight, AF. Dissection of CENP-C-directed centromere and kinetochore assembly. Mol Biol Cell. (2009) 20(19): p. 4246-55.

68. Przewloka, MR, Venkei, Z, Bolanos-Garcia, VM, Debski, J, Dadlez, M, and Glover, DM. CENP-C is a structural platform for kinetochore assembly. Curr Biol. (2011) 21(5): p. 399-405.

69. Screpanti, E, De Antoni, A, Alushin, GM, Petrovic, A, Melis, T, Nogales, E, and Musacchio, A. Direct binding of Cenp-C to the Mis12 complex joins the inner and outer kinetochore. Curr Biol. (2011) 21(5): p. 391-8.

70. Suzuki, A, Hori, T, Nishino, T, Usukura, J, Miyagi, A, Morikawa, K, and Fukagawa, T. Spindle microtubules generate tension-dependent changes in the distribution of inner kinetochore proteins. J Cell Biol. (2011) 193(1): p. 125-40.

71. Gascoigne, KE, Takeuchi, K, Suzuki, A, Hori, T, Fukagawa, T, and Cheeseman, IM. Induced ectopic kinetochore assembly bypasses the requirement for CENP-A nucleosomes. Cell. (2011) 145(3): p. 410-22.

72. Schleiffer, A, Maier, M, Litos, G, Lampert, F, Hornung, P, Mechtler, K, and Westermann, S. CENP-T proteins are conserved centromere receptors of the Ndc80 complex. Nat Cell Biol. (2012).

73. Cheeseman, IM, Hori, T, Fukagawa, T, and Desai, A. KNL1 and the CENP$\mathrm{H} / \mathrm{I} / \mathrm{K}$ complex coordinately direct kinetochore assembly in vertebrates. Mol Biol Cell. (2008) 19(2): p. 587-94.

74. Amaro, AC, Samora, CP, Holtackers, R, Wang, E, Kingston, IJ, Alonso, M, Lampson, M, McAinsh, AD, and Meraldi, P. Molecular control of kinetochore-microtubule dynamics and chromosome oscillations. Nat Cell Biol. (2010) 12(4): p. 319-29.

75. Hori, T, Okada, M, Maenaka, K, and Fukagawa, T. CENP-O class proteins form a stable complex and are required for proper kinetochore function. Mol Biol Cell. (2008) 19(3): p. 843-54.

76. Probst, AV, Dunleavy, E, and Almouzni, G. Epigenetic inheritance during the cell cycle. Nat Rev Mol Cell Biol. (2009) 10(3): p. 192-206.

77. Ransom, M, Dennehey, BK, and Tyler, JK. Chaperoning histones during DNA replication and repair. Cell. (2010) 140(2): p. 183-95.

78. Jansen, LE, Black, BE, Foltz, DR, and Cleveland, DW. Propagation of centromeric chromatin requires exit from mitosis. J Cell Biol. (2007) 176(6): p. 795-805. 
79. Dunleavy, EM, Almouzni, G, and Karpen, GH. H3.3 is deposited at centromeres in $\mathrm{S}$ phase as a placeholder for newly assembled CENP-A in G phase. Nucleus. (2011) 2(2): p. 146-57.

80. Van Hooser, AA, Ouspenski, II, Gregson, HC, Starr, DA, Yen, TJ, Goldberg, ML, Yokomori, K, Earnshaw, WC, Sullivan, KF, and Brinkley, BR. Specification of kinetochore-forming chromatin by the histone H3 variant CENP-A. J Cell Sci. (2001) 114(Pt 19): p. 3529-42.

81. Moreno-Moreno, $\mathrm{O}$, Torras-Llort, $\mathrm{M}$, and Azorin, F. Proteolysis restricts localization of CID, the centromere-specific histone $\mathrm{H} 3$ variant of Drosophila, to centromeres. Nucleic Acids Res. (2006) 34(21): p. 624755.

82. Heun, P, Erhardt, S, Blower, MD, Weiss, S, Skora, AD, and Karpen, GH. Mislocalization of the Drosophila centromere-specific histone CID promotes formation of functional ectopic kinetochores. Dev Cell. (2006) 10(3): p. 303-15.

83. Foltz, DR, Jansen, LE, Bailey, AO, Yates, JR, 3rd, Bassett, EA, Wood, S, Black, BE, and Cleveland, DW. Centromere-specific assembly of CENP-a nucleosomes is mediated by HJURP. Cell. (2009) 137(3): p. 472-84.

84. Shelby, RD, Monier, K, and Sullivan, KF. Chromatin assembly at kinetochores is uncoupled from DNA replication. J Cell Biol. (2000) 151(5): p. 1113-8.

85. Shelby, RD, Vafa, O, and Sullivan, KF. Assembly of CENP-A into centromeric chromatin requires a cooperative array of nucleosomal DNA contact sites. J Cell Biol. (1997) 136(3): p. 501-13.

86. Bui, M, Dimitriadis, EK, Hoischen, C, An, E, Quenet, D, Giebe, S, NitaLazar, A, Diekmann, S, and Dalal, Y. Cell-cycle-dependent structural transitions in the human CENP-A nucleosome in vivo. Cell. (2012) 150(2): p. 317-26.

87. Shivaraju, M, Unruh, JR, Slaughter, BD, Mattingly, M, Berman, J, and Gerton, JL. Cell-cycle-coupled structural oscillation of centromeric nucleosomes in yeast. Cell. (2012) 150(2): p. 304-16.

88. Hemmerich, P, Weidtkamp-Peters, S, Hoischen, C, Schmiedeberg, L, Erliandri, I, and Diekmann, S. Dynamics of inner kinetochore assembly and maintenance in living cells. J Cell Biol. (2008) 180(6): p. 1101-14.

89. Hellwig, D, Emmerth, S, Ulbricht, T, Doring, V, Hoischen, C, Martin, R, Samora, CP, McAinsh, AD, Carroll, CW, Straight, AF, Meraldi, P, and Diekmann, S. Dynamics of CENP-N kinetochore binding during the cell cycle. J Cell Sci. (2011) 124(Pt 22): p. 3871-83.

90. Fujita, Y, Hayashi, T, Kiyomitsu, T, Toyoda, Y, Kokubu, A, Obuse, C, and Yanagida, M. Priming of centromere for CENP-A recruitment by human 
hMis18alpha, hMis18beta, and M18BP1. Dev Cell. (2007) 12(1): p. 1730.

91. Hayashi, T, Fujita, Y, Iwasaki, O, Adachi, Y, Takahashi, K, and Yanagida, M. Mis16 and Mis18 are required for CENP-A loading and histone deacetylation at centromeres. Cell. (2004) 118(6): p. 715-29.

92. Maddox, PS, Hyndman, F, Monen, J, Oegema, K, and Desai, A. Functional genomics identifies a Myb domain-containing protein family required for assembly of CENP-A chromatin. J Cell Biol. (2007) 176(6): p. 75763.

93. Schuh, M, Lehner, CF, and Heidmann, S. Incorporation of Drosophila CID/CENP-A and CENP-C into centromeres during early embryonic anaphase. Curr Biol. (2007) 17(3): p. 237-43.

94. Mellone, BG, Grive, KJ, Shteyn, V, Bowers, SR, Oderberg, I, and Karpen, $\mathrm{GH}$. Assembly of Drosophila centromeric chromatin proteins during mitosis. PLoS Genet. (2011) 7(5): p. e1002068.

95. Erhardt, S, Mellone, BG, Betts, CM, Zhang, W, Karpen, GH, and Straight, AF. Genome-wide analysis reveals a cell cycle-dependent mechanism controlling centromere propagation. J Cell Biol. (2008) 183(5): p. 80518.

96. Grosskortenhaus, R and Sprenger, F. Rca1 inhibits APC-Cdh1(Fzr) and is required to prevent cyclin degradation in G2. Dev Cell. (2002) 2(1): p. 29-40.

97. Uhlmann, F, Bouchoux, C, and Lopez-Aviles, S. A quantitative model for cyclin-dependent kinase control of the cell cycle: revisited. Philos Trans R Soc Lond B Biol Sci. (2011) 366(1584): p. 3572-83.

98. Obaya, AJ and Sedivy, JM. Regulation of cyclin-Cdk activity in mammalian cells. Cell Mol Life Sci. (2002) 59(1): p. 126-42.

99. Dambacher, S, Deng, W, Hahn, M, Sadic, D, Frohlich, J, Nuber, A, Hoischen, C, Diekmann, S, Leonhardt, H, and Schotta, G. CENP-C facilitates the recruitment of M18BP1 to centromeric chromatin. Nucleus. (2012) 3(1).

100. Moree, B, Meyer, CB, Fuller, CJ, and Straight, AF. CENP-C recruits M18BP1 to centromeres to promote CENP-A chromatin assembly. J Cell Biol. (2011) 194(6): p. 855-71.

101. Orr, B and Sunkel, CE. Drosophila CENP-C is essential for centromere identity. Chromosoma. (2011) 120(1): p. 83-96.

102. Goshima, G, Kiyomitsu, T, Yoda, K, and Yanagida, M. Human centromere chromatin protein hMis12, essential for equal segregation, is independent of CENP-A loading pathway. J Cell Biol. (2003) 160(1): p. 25-39. 
103. Cuylen, $\mathrm{S}$ and Haering, CH. Deciphering condensin action during chromosome segregation. Trends Cell Biol. (2011) 21(9): p. 552-9.

104. Yong-Gonzalez, V, Wang, BD, Butylin, P, Ouspenski, I, and Strunnikov, A. Condensin function at centromere chromatin facilitates proper kinetochore tension and ensures correct mitotic segregation of sister chromatids. Genes Cells. (2007) 12(9): p. 1075-90.

105. Bernad, R, Sanchez, P, Rivera, T, Rodriguez-Corsino, M, Boyarchuk, E, Vassias, I, Ray-Gallet, D, Arnaoutov, A, Dasso, M, Almouzni, G, and Losada, A. Xenopus HJURP and condensin II are required for CENP-A assembly. J Cell Biol. (2011) 192(4): p. 569-82.

106. Samoshkin, A, Arnaoutov, A, Jansen, LE, Ouspenski, I, Dye, L, Karpova, T, McNally, J, Dasso, M, Cleveland, DW, and Strunnikov, A. Human condensin function is essential for centromeric chromatin assembly and proper sister kinetochore orientation. PLoS One. (2009) 4(8): p. e6831.

107. Tada, K, Susumu, H, Sakuno, T, and Watanabe, Y. Condensin association with histone H2A shapes mitotic chromosomes. Nature. (2011) 474(7352): p. 477-83.

108. Gopalakrishnan, S, Sullivan, BA, Trazzi, S, Della Valle, G, and Robertson, KD. DNMT3B interacts with constitutive centromere protein CENP-C to modulate DNA methylation and the histone code at centromeric regions. Hum Mol Genet. (2009) 18(17): p. 3178-93.

109. Kim, IS, Lee, M, Park, KC, Jeon, Y, Park, JH, Hwang, EJ, Jeon, TI, Ko, S, Lee, $\mathrm{H}, \mathrm{Baek}, \mathrm{SH}$, and Kim, KI. Roles of Mis18alpha in epigenetic regulation of centromeric chromatin and CENP-A loading. Mol Cell. (2012) 46(3): p. 260-73.

110. Tsumura, A, Hayakawa, T, Kumaki, Y, Takebayashi, S, Sakaue, M, Matsuoka, C, Shimotohno, K, Ishikawa, F, Li, E, Ueda, HR, Nakayama, J, and Okano, M. Maintenance of self-renewal ability of mouse embryonic stem cells in the absence of DNA methyltransferases Dnmt1, Dnmt3a and Dnmt3b. Genes Cells. (2006) 11(7): p. 805-14.

111. Zhang, D, Martyniuk, CJ, and Trudeau, VL. SANTA domain: a novel conserved protein module in Eukaryota with potential involvement in chromatin regulation. Bioinformatics. (2006) 22(20): p. 2459-62.

112. Boyer, LA, Langer, MR, Crowley, KA, Tan, S, Denu, JM, and Peterson, CL. Essential role for the SANT domain in the functioning of multiple chromatin remodeling enzymes. Mol Cell. (2002) 10(4): p. 935-42.

113. Horton, JR, Elgar, SJ, Khan, SI, Zhang, X, Wade, PA, and Cheng, X. Structure of the SANT domain from the Xenopus chromatin remodeling factor ISWI. Proteins. (2007) 67(4): p. 1198-202. 
114. Ogata, K, Morikawa, S, Nakamura, H, Sekikawa, A, Inoue, T, Kanai, H, Sarai, A, Ishii, S, and Nishimura, Y. Solution structure of a specific DNA complex of the Myb DNA-binding domain with cooperative recognition helices. Cell. (1994) 79(4): p. 639-48.

115. Guenther, MG, Barak, O, and Lazar, MA. The SMRT and N-CoR corepressors are activating cofactors for histone deacetylase 3. Mol Cell Biol. (2001) 21(18): p. 6091-101.

116. Sterner, DE, Wang, X, Bloom, MH, Simon, GM, and Berger, SL. The SANT domain of Ada2 is required for normal acetylation of histones by the yeast SAGA complex. J Biol Chem. (2002) 277(10): p. 8178-86.

117. You, A, Tong, JK, Grozinger, CM, and Schreiber, SL. CoREST is an integral component of the CoREST- human histone deacetylase complex. Proc Natl Acad Sci U S A. (2001) 98(4): p. 1454-8.

118. Loyola, A and Almouzni, G. Histone chaperones, a supporting role in the limelight. Biochim Biophys Acta. (2004) 1677(1-3): p. 3-11.

119. Philpott, A, Krude, T, and Laskey, RA. Nuclear chaperones. Semin Cell Dev Biol. (2000) 11(1): p. 7-14.

120. Shuaib, M, Ouararhni, K, Dimitrov, S, and Hamiche, A. HJURP binds CENP-A via a highly conserved N-terminal domain and mediates its deposition at centromeres. Proc Natl Acad Sci U S A. (2010) 107(4): p. 1349-54.

121. Camahort, R, Li, B, Florens, L, Swanson, SK, Washburn, MP, and Gerton, JL. Scm 3 is essential to recruit the histone h3 variant cse 4 to centromeres and to maintain a functional kinetochore. Mol Cell. (2007) 26(6): p. 853-65.

122. Stoler, S, Rogers, K, Weitze, S, Morey, L, Fitzgerald-Hayes, M, and Baker, RE. Scm3, an essential Saccharomyces cerevisiae centromere protein required for G2/M progression and Cse4 localization. Proc Natl Acad Sci U S A. (2007) 104(25): p. 10571-6.

123. Kato, T, Sato, N, Hayama, S, Yamabuki, T, Ito, T, Miyamoto, M, Kondo, S, Nakamura, Y, and Daigo, Y. Activation of Holliday junction recognizing protein involved in the chromosomal stability and immortality of cancer cells. Cancer Res. (2007) 67(18): p. 8544-53.

124. Chen, CC, Dechassa, ML, Bettini, E, Ledoux, MB, Belisario, C, Heun, P, Luger, $\mathrm{K}$, and Mellone, BG. CAL1 is the Drosophila CENP--a assembly factor. J Cell Biol. (2014) 204(3): p. 313-29.

125. Dechassa, ML, Wyns, K, Li, M, Hall, MA, Wang, MD, and Luger, K. Structure and Scm3-mediated assembly of budding yeast centromeric nucleosomes. Nat Commun. (2011) 2: p. 313. 
126. Camahort, R, Shivaraju, M, Mattingly, M, Li, B, Nakanishi, S, Zhu, D, Shilatifard, A, Workman, JL, and Gerton, JL. Cse4 is part of an octameric nucleosome in budding yeast. Mol Cell. (2009) 35(6): p. 794-805.

127. Kingston, IJ, Yung, JS, and Singleton, MR. Biophysical characterization of the centromere-specific nucleosome from budding yeast. J Biol Chem. (2011) 286(5): p. 4021-6.

128. Shivaraju, M, Camahort, R, Mattingly, M, and Gerton, JL. Scm3 is a centromeric nucleosome assembly factor. J Biol Chem. (2011) 286(14): p. 12016-23.

129. Hu, H, Liu, Y, Wang, M, Fang, J, Huang, H, Yang, N, Li, Y, Wang, J, Yao, X, Shi, Y, Li, G, and Xu, RM. Structure of a CENP-A-histone H4 heterodimer in complex with chaperone HJURP. Genes Dev. (2011) 25(9): p. 901-6.

130. Cho, US and Harrison, SC. Recognition of the centromere-specific histone Cse4 by the chaperone Scm3. Proc Natl Acad Sci U S A. (2011) 108(23): p. 9367-71.

131. Zhou, Z, Feng, H, Zhou, BR, Ghirlando, R, Hu, K, Zwolak, A, Miller Jenkins, LM, Xiao, H, Tjandra, N, Wu, C, and Bai, Y. Structural basis for recognition of centromere histone variant $\mathrm{CenH} 3$ by the chaperone Scm3. Nature. (2011) 472(7342): p. 234-7.

132. Perpelescu, M, Nozaki, N, Obuse, C, Yang, H, and Yoda, K. Active establishment of centromeric CENP-A chromatin by RSF complex. J Cell Biol. (2009) 185(3): p. 397-407.

133. Lagana, A, Dorn, JF, De Rop, V, Ladouceur, AM, Maddox, AS, and Maddox, PS. A small GTPase molecular switch regulates epigenetic centromere maintenance by stabilizing newly incorporated CENP-A. Nat Cell Biol. (2010) 12(12): p. 1186-93.

134. Choi, ES, Stralfors, A, Castillo, AG, Durand-Dubief, M, Ekwall, K, and Allshire, RC. Identification of noncoding transcripts from within CENPA chromatin at fission yeast centromeres. J Biol Chem. (2011) 286(26): p. 23600-7.

135. Topp, CN, Zhong, CX, and Dawe, RK. Centromere-encoded RNAs are integral components of the maize kinetochore. Proc Natl Acad Sci U S A. (2004) 101(45): p. 15986-91.

136. Chan, FL, Marshall, OJ, Saffery, R, Won Kim, B, Earle, E, Choo, KH, and Wong, LH. Active transcription and essential role of RNA polymerase II at the centromere during mitosis. Proc Natl Acad Sci U S A. (2012) 109(6): p. 1979-84.

137. Wong, LH, Brettingham-Moore, KH, Chan, L, Quach, JM, Anderson, MA, Northrop, EL, Hannan, R, Saffery, R, Shaw, ML, Williams, E, and Choo, $\mathrm{KH}$. Centromere RNA is a key component for the assembly of 
nucleoproteins at the nucleolus and centromere. Genome Res. (2007) 17(8): p. 1146-60.

138. Chueh, AC, Northrop, EL, Brettingham-Moore, KH, Choo, KH, and Wong, LH. LINE retrotransposon RNA is an essential structural and functional epigenetic component of a core neocentromeric chromatin. PLoS Genet. (2009) 5(1): p. e1000354.

139. Bergmann, JH, Jakubsche, JN, Martins, NM, Kagansky, A, Nakano, M, Kimura, H, Kelly, DA, Turner, BM, Masumoto, H, Larionov, V, and Earnshaw, WC. Epigenetic engineering: histone H3K9 acetylation is compatible with kinetochore structure and function. J Cell Sci. (2012) 125(Pt 2): p. 411-21.

140. Du, Y, Topp, CN, and Dawe, RK. DNA binding of centromere protein C (CENPC) is stabilized by single-stranded RNA. PLoS Genet. (2010) 6(2): p. e1000835.

141. Izuta, $\mathrm{H}$, Ikeno, $\mathrm{M}$, Suzuki, $\mathrm{N}$, Tomonaga, $\mathrm{T}$, Nozaki, $\mathrm{N}$, Obuse, $\mathrm{C}, \mathrm{Kisu}, \mathrm{Y}$, Goshima, N, Nomura, F, Nomura, N, and Yoda, K. Comprehensive analysis of the ICEN (Interphase Centromere Complex) components enriched in the CENP-A chromatin of human cells. Genes Cells. (2006) 11(6): p. 673-84.

142. Okada, M, Okawa, K, Isobe, T, and Fukagawa, T. CENP-H-containing complex facilitates centromere deposition of CENP-A in cooperation with FACT and CHD1. Mol Biol Cell. (2009) 20(18): p. 3986-95.

143. Walfridsson, J, Bjerling, P, Thalen, M, Yoo, EJ, Park, SD, and Ekwall, K. The CHD remodeling factor Hrp1 stimulates CENP-A loading to centromeres. Nucleic Acids Res. (2005) 33(9): p. 2868-79.

144. Hewawasam, G, Shivaraju, M, Mattingly, M, Venkatesh, S, MartinBrown, S, Florens, L, Workman, JL, and Gerton, JL. Psh1 is an E3 ubiquitin ligase that targets the centromeric histone variant Cse4. Mol Cell. (2010) 40(3): p. 444-54.

145. Ranjitkar, P, Press, MO, Yi, X, Baker, R, MacCoss, MJ, and Biggins, S. An E3 ubiquitin ligase prevents ectopic localization of the centromeric histone H3 variant via the centromere targeting domain. Mol Cell. (2010) 40(3): p. 455-64.

146. Moreno-Moreno, O, Medina-Giro, S, Torras-Llort, M, and Azorin, F. The F Box Protein Partner of Paired Regulates Stability of Drosophila Centromeric Histone H3, CenH3(CID). Curr Biol. (2011) 21(17): p. 1488-93.

147. Lomonte, P, Sullivan, KF, and Everett, RD. Degradation of nucleosomeassociated centromeric histone H3-like protein CENP-A induced by 
herpes simplex virus type 1 protein ICP0. J Biol Chem. (2001) 276(8): p. 5829-35.

148. Collins, KA, Furuyama, S, and Biggins, S. Proteolysis contributes to the exclusive centromere localization of the yeast Cse4/CENP-A histone H3 variant. Curr Biol. (2004) 14(21): p. 1968-72.

149. Gkikopoulos, T, Singh, V, Tsui, K, Awad, S, Renshaw, MJ, Scholfield, P, Barton, GJ, Nislow, C, Tanaka, TU, and Owen-Hughes, T. The SWI/SNF complex acts to constrain distribution of the centromeric histone variant Cse4. EMBO J. (2011) 30(10): p. 1919-27.

150. Hamiche, A and Shuaib, M. Chaperoning the histone $\mathrm{H} 3$ family. Biochim Biophys Acta. (2012) 1819(3-4): p. 230-7.

151. Wang, J, Liu, X, Dou, Z, Chen, L, Jiang, H, Fu, C, Fu, G, Liu, D, Zhang, J, Zhu, T, Fang, J, Zang, J, Cheng, J, Teng, M, Ding, X, and Yao, X. Mitotic Regulator Mis18beta Interacts with and Specifies the Centromeric Assembly of Molecular Chaperone Holliday Junction Recognition Protein (HJURP). J Biol Chem. (2014) 289(12): p. 8326-36.

152. McDonnell, AV, Jiang, T, Keating, AE, and Berger, B. Paircoil2: improved prediction of coiled coils from sequence. Bioinformatics. (2006) 22(3): p. 356-8.

153. Mason, JM, Schmitz, MA, Muller, KM, and Arndt, KM. Semirational design of Jun-Fos coiled coils with increased affinity: Universal implications for leucine zipper prediction and design. Proc Natl Acad Sci USA. (2006) 103(24): p. 8989-94.

154. Biomatters, Geneious Pro Version 5.0.4 created by Biomatters. Available from: http://www.geneious.com/. 2005-2010.

155. Wang, J, Liu, X, Dou, Z, Chen, L, Jiang, H, Fu, C, Fu, G, Liu, D, Zhang, J, Zhu, T, Fang, J, Zang, J, Cheng, J, Teng, M, Ding, X, and Yao, X. Mitotic regulator Mis18beta interacts with and specifies the centromeric assembly of molecular chaperone HJURP. J Biol Chem. (2014).

156. Pidoux, AL, Choi, ES, Abbott, JK, Liu, X, Kagansky, A, Castillo, AG, Hamilton, GL, Richardson, W, Rappsilber, J, He, X, and Allshire, RC. Fission yeast Scm3: A CENP-A receptor required for integrity of subkinetochore chromatin. Mol Cell. (2009) 33(3): p. 299-311.

157. Williams, JS, Hayashi, T, Yanagida, M, and Russell, P. Fission yeast Scm3 mediates stable assembly of Cnp1/CENP-A into centromeric chromatin. Mol Cell. (2009) 33(3): p. 287-98.

158. Xiao, H, Mizuguchi, G, Wisniewski, J, Huang, Y, Wei, D, and Wu, C. Nonhistone $\mathrm{Scm} 3$ binds to AT-rich DNA to organize atypical centromeric nucleosome of budding yeast. Mol Cell. (2011) 43(3): p. 369-80. 
159. Phair, RD, Gorski, SA, and Misteli, T. Measurement of dynamic protein binding to chromatin in vivo, using photobleaching microscopy.

Methods Enzymol. (2004) 375: p. 393-414.

160. Stellfox, ME, Bailey, AO, and Foltz, DR. Putting CENP-A in its place. Cell Mol Life Sci. (2012).

161. Cleveland, DW, Mao, Y, and Sullivan, KF. Centromeres and kinetochores: from epigenetics to mitotic checkpoint signaling. Cell. (2003) 112(4): p. 407-21.

162. Wu, RS and Bonner, WM. Separation of basal histone synthesis from Sphase histone synthesis in dividing cells. Cell. (1981) 27(2 Pt 1): p. 321-30.

163. Kim, IS, Lee, M, Park, JH, Jeon, R, Baek, SH, and Kim, KI. betaTrCPmediated ubiquitylation regulates protein stability of Mis18beta in a cell cycle-dependent manner. Biochem Biophys Res Commun. (2013).

164. Silva, MC, Bodor, DL, Stellfox, ME, Martins, NM, Hochegger, H, Foltz, DR, and Jansen, LE. Cdk activity couples epigenetic centromere inheritance to cell cycle progression. Dev Cell. (2012) 22(1): p. 52-63.

165. Lermontova, I, Kuhlmann, M, Friedel, S, Rutten, T, Heckmann, S, Sandmann, M, Demidov, D, Schubert, V, and Schubert, I. Arabidopsis KINETOCHORE NULL2 Is an Upstream Component for Centromeric Histone H3 Variant cenH3 Deposition at Centromeres. Plant Cell. (2013) 25(9): p. 3389-404.

166. Janicki, SM and Spector, DL. Nuclear choreography: interpretations from living cells. Curr Opin Cell Biol. (2003) 15(2): p. 149-57.

167. Zasadzinska, E, Barnhart-Dailey, MC, Kuich, PH, and Foltz, DR. Dimerization of the CENP-A assembly factor HJURP is required for centromeric nucleosome deposition. EMBO J. (2013) 32(15): p. 211324.

168. Lanini, L and McKeon, F. Domains required for CENP-C assembly at the kinetochore. Mol Biol Cell. (1995) 6(8): p. 1049-59.

169. Song, K, Gronemeyer, B, Lu, W, Eugster, E, and Tomkiel, JE. Mutational analysis of the central centromere targeting domain of human centromere protein C, (CENP-C). Exp Cell Res. (2002) 275(1): p. 81-91.

170. Trazzi, S, Bernardoni, R, Diolaiti, D, Politi, V, Earnshaw, WC, Perini, G, and Della Valle, G. In vivo functional dissection of human inner kinetochore protein CENP-C. J Struct Biol. (2002) 140(1-3): p. 39-48.

171. Yang, CH, Tomkiel, J, Saitoh, H, Johnson, DH, and Earnshaw, WC. Identification of overlapping DNA-binding and centromere-targeting domains in the human kinetochore protein CENP-C. Mol Cell Biol. (1996) 16(7): p. 3576-86. 
172. Petronczki, M, Lenart, P, and Peters, JM. Polo on the Rise-from Mitotic Entry to Cytokinesis with Plk1. Dev Cell. (2008) 14(5): p. 646-59.

173. Liu, Z, Ren, J, Cao, J, He, J, Yao, X, Jin, C, and Xue, Y. Systematic analysis of the Plk-mediated phosphoregulation in eukaryotes. Brief Bioinform. (2013) 14(3): p. 344-60.

174. Phansalkar, R, Lapierre, P, and Mellone, BG. Evolutionary insights into the role of the essential centromere protein CAL1 in Drosophila. Chromosome Res. (2012) 20(5): p. 493-504.

175. Schneider, CA, Rasband, WS, and Eliceiri, KW. NIH Image to ImageJ: 25 years of image analysis. Nat Methods. (2012) 9(7): p. 671-5.

176. Almouzni, G and Probst, AV. Heterochromatin maintenance and establishment: lessons from the mouse pericentromere. Nucleus. (2011) 2(5): p. 332-8.

177. Guenatri, M, Bailly, D, Maison, C, and Almouzni, G. Mouse centric and pericentric satellite repeats form distinct functional heterochromatin. $J$ Cell Biol. (2004) 166(4): p. 493-505.

178. Fukagawa, T, Nogami, M, Yoshikawa, M, Ikeno, M, Okazaki, T, Takami, Y, Nakayama, T, and Oshimura, M. Dicer is essential for formation of the heterochromatin structure in vertebrate cells. Nat Cell Biol. (2004) 6(8): p. 784-91.

179. Nonaka, N, Kitajima, T, Yokobayashi, S, Xiao, G, Yamamoto, M, Grewal, SI, and Watanabe, Y. Recruitment of cohesin to heterochromatic regions by Swi6/HP1 in fission yeast. Nat Cell Biol. (2002) 4(1): p. 8993.

180. Melcher, M, Schmid, M, Aagaard, L, Selenko, P, Laible, G, and Jenuwein, T. Structure-function analysis of SUV39H1 reveals a dominant role in heterochromatin organization, chromosome segregation, and mitotic progression. Mol Cell Biol. (2000) 20(10): p. 3728-41.

181. Jiang, YL, Rigolet, M, Bourc'his, D, Nigon, F, Bokesoy, I, Fryns, JP, Hulten, $M$, Jonveaux, $\mathrm{P}$, Maraschio, $\mathrm{P}$, Megarbane, A, Moncla, A, and ViegasPequignot, E. DNMT3B mutations and DNA methylation defect define two types of ICF syndrome. Hum Mutat. (2005) 25(1): p. 56-63.

182. Maison, C, Bailly, D, Peters, AH, Quivy, JP, Roche, D, Taddei, A, Lachner, $M$, Jenuwein, T, and Almouzni, G. Higher-order structure in pericentric heterochromatin involves a distinct pattern of histone modification and an RNA component. Nat Genet. (2002) 30(3): p. 329-34.

183. Bernard, P, Maure, JF, Partridge, JF, Genier, S, Javerzat, JP, and Allshire, RC. Requirement of heterochromatin for cohesion at centromeres. Science. (2001) 294(5551): p. 2539-42. 
184. Martens, JH, O'Sullivan, RJ, Braunschweig, U, Opravil, S, Radolf, M, Steinlein, $\mathrm{P}$, and Jenuwein, $\mathrm{T}$. The profile of repeat-associated histone lysine methylation states in the mouse epigenome. EMBO J. (2005) 24(4): p. 800-12.

185. Peters, AH, O'Carroll, D, Scherthan, H, Mechtler, K, Sauer, S, Schofer, C, Weipoltshammer, K, Pagani, M, Lachner, M, Kohlmaier, A, Opravil, S, Doyle, M, Sibilia, M, and Jenuwein, T. Loss of the Suv39h histone methyltransferases impairs mammalian heterochromatin and genome stability. Cell. (2001) 107(3): p. 323-37.

186. Nurse, P and Bissett, Y. Gene required in G1 for commitment to cell cycle and in G2 for control of mitosis in fission yeast. Nature. (1981) 292(5823): p. 558-60.

187. James, TC and Elgin, SC. Identification of a nonhistone chromosomal protein associated with heterochromatin in Drosophila melanogaster and its gene. Mol Cell Biol. (1986) 6(11): p. 3862-72.

188. Wreggett, KA, Hill, F, James, PS, Hutchings, A, Butcher, GW, and Singh, PB. A mammalian homologue of Drosophila heterochromatin protein 1 (HP1) is a component of constitutive heterochromatin. Cytogenet Cell Genet. (1994) 66(2): p. 99-103.

189. Minc, E, Allory, Y, Worman, HJ, Courvalin, JC, and Buendia, B. Localization and phosphorylation of HP1 proteins during the cell cycle in mammalian cells. Chromosoma. (1999) 108(4): p. 220-34.

190. Prasanth, SG, Prasanth, KV, Siddiqui, K, Spector, DL, and Stillman, B. Human Orc2 localizes to centrosomes, centromeres and heterochromatin during chromosome inheritance. EMBO J. (2004) 23(13): p. 2651-63.

191. Chakraborty, A, Prasanth, KV, and Prasanth, SG. Dynamic phosphorylation of HP1alpha regulates mitotic progression in human cells. Nat Commun. (2014) 5: p. 3445.

192. Hori, T, Shang, WH, Takeuchi, K, and Fukagawa, T. The CCAN recruits CENP-A to the centromere and forms the structural core for kinetochore assembly. J Cell Biol. (2013) 200(1): p. 45-60.

193. Lacoste, N, Woolfe, A, Tachiwana, H, Garea, AV, Barth, T, Cantaloube, S, Kurumizaka, H, Imhof, A, and Almouzni, G. Mislocalization of the Centromeric Histone Variant CenH3/CENP-A in Human Cells Depends on the Chaperone DAXX. Mol Cell. (2014) 53(4): p. 631-44.

194. Boyer, LA, Latek, RR, and Peterson, CL. The SANT domain: a unique histone-tail-binding module? Nat Rev Mol Cell Biol. (2004) 5(2): p. 15863. 
195. Hecht, A, Laroche, T, Strahl-Bolsinger, S, Gasser, SM, and Grunstein, M. Histone $\mathrm{H} 3$ and $\mathrm{H} 4 \mathrm{~N}$-termini interact with SIR3 and SIR4 proteins: a molecular model for the formation of heterochromatin in yeast. Cell. (1995) 80(4): p. 583-92.

196. Smith, MM and Stirling, VB. Histone H3 and H4 gene deletions in Saccharomyces cerevisiae. J Cell Biol. (1988) 106(3): p. 557-66. 


\section{Appendix:}

\section{Antibodies and Plasmids}




\section{Antibody Dilutions}

\section{Primary Antibodies}

\begin{tabular}{|c|c|c|c|c|}
\hline Protein & Host & Label Information & Dilution & Application \\
\hline CENP-A & Mouse & ab13939, Abcam & $\begin{array}{c}1: 1000 \\
(1 \mu \mathrm{g} / \mathrm{mL})\end{array}$ & IF \& WB \\
\hline CENP-C & Mouse & Serum (Custom) & $\begin{array}{c}1: 3000- \\
1: 5000\end{array}$ & IF \\
\hline CENP-T & Rabbit & $\begin{array}{l}\text { D. Cleveland } \\
\text { Ludwig Institute for } \\
\text { Cancer Research }\end{array}$ & $\begin{array}{c}1: 1000 \\
(1: 2 \text { in glycerol) }\end{array}$ & IF \\
\hline GFP & Rabbit & 3403 (Custom Covance) & $1: 1000$ & WB \\
\hline His & Rabbit & $\begin{array}{c}\mathrm{H}-15 \\
\text { (Santa Cruz, sc-803) } \\
\end{array}$ & $1: 1000$ & WB \\
\hline HJURP & Rabbit & 3399 & $1: 1000$ & WB \\
\hline Mis18BP1 & Rabbit & BL10286/A302-825A & $1: 5000$ & WB \\
\hline Mis18BP1 & Rabbit & BL10285/A302-824A & $\begin{array}{c}1: 1000- \\
1: 2000\end{array}$ & WB \\
\hline Mis18 $\beta$ & Rabbit & BL10295 & $1: 500-1: 1000$ & WB \\
\hline Myc & Mouse & $\begin{array}{c}\text { 9E10 } \\
(\text { Santa Cruz, sc-40) }\end{array}$ & $1: 250$ & WB \\
\hline Tubulin & Mouse & AA2 (Hybridoma) & $1: 1000$ & WB \\
\hline CENP-I & Rabbit & $\begin{array}{l}\text { Gift from P.T. Stukenberg } \\
\text { (Dan Matson) }\end{array}$ & $1: 1000$ & IF \& WB \\
\hline CENP-H & Rabbit & $\begin{array}{l}\text { Gift from P.T. Stukenberg } \\
\text { (Dan Matson) }\end{array}$ & $1: 1000$ & WB \\
\hline
\end{tabular}

\section{Secondary Antibodies}

\begin{tabular}{|c|c|c|}
\hline Protein & Label & Dilution \\
\hline G $\boldsymbol{\alpha} \mathbf{M}$ & Alexa-647 (Jackson) & $\begin{array}{c}1: 2000-1: 4000 \\
(1: 2 \text { in glycerol) }\end{array}$ \\
\hline G $\boldsymbol{\alpha} \mathbf{M}$ & Cy3 (Jackson) & $1: 2000-1: 4000$ \\
\hline $\mathbf{G} \boldsymbol{\alpha} \mathbf{R}$ & Cy5 (Jackson) & $\begin{array}{c}1: 1000 \\
(1: 2 \text { in glycerol) }\end{array}$ \\
\hline D $\boldsymbol{\alpha} \mathbf{R}$ & Cy3 (Jackson) & $1: 1000$ \\
\hline $\mathbf{G} \boldsymbol{\alpha} \mathbf{M}$ & HRP (Jackson) & $1: 10000$ \\
\hline $\mathbf{G} \boldsymbol{\alpha} \mathbf{R}$ & HRP (Jackson) & $1: 10000$ \\
\hline D $\boldsymbol{\alpha}$ H & HRP (Jackson) & $1: 5000$ \\
\hline $\mathbf{D} \boldsymbol{\alpha} \mathbf{R}$ & HRP (Bethyl Reliablot) & $1: 5000$ \\
\hline
\end{tabular}




\section{Plasmid List}

***Plasmid maps in electronic laboratory copy***

Plasmid list on following pages 


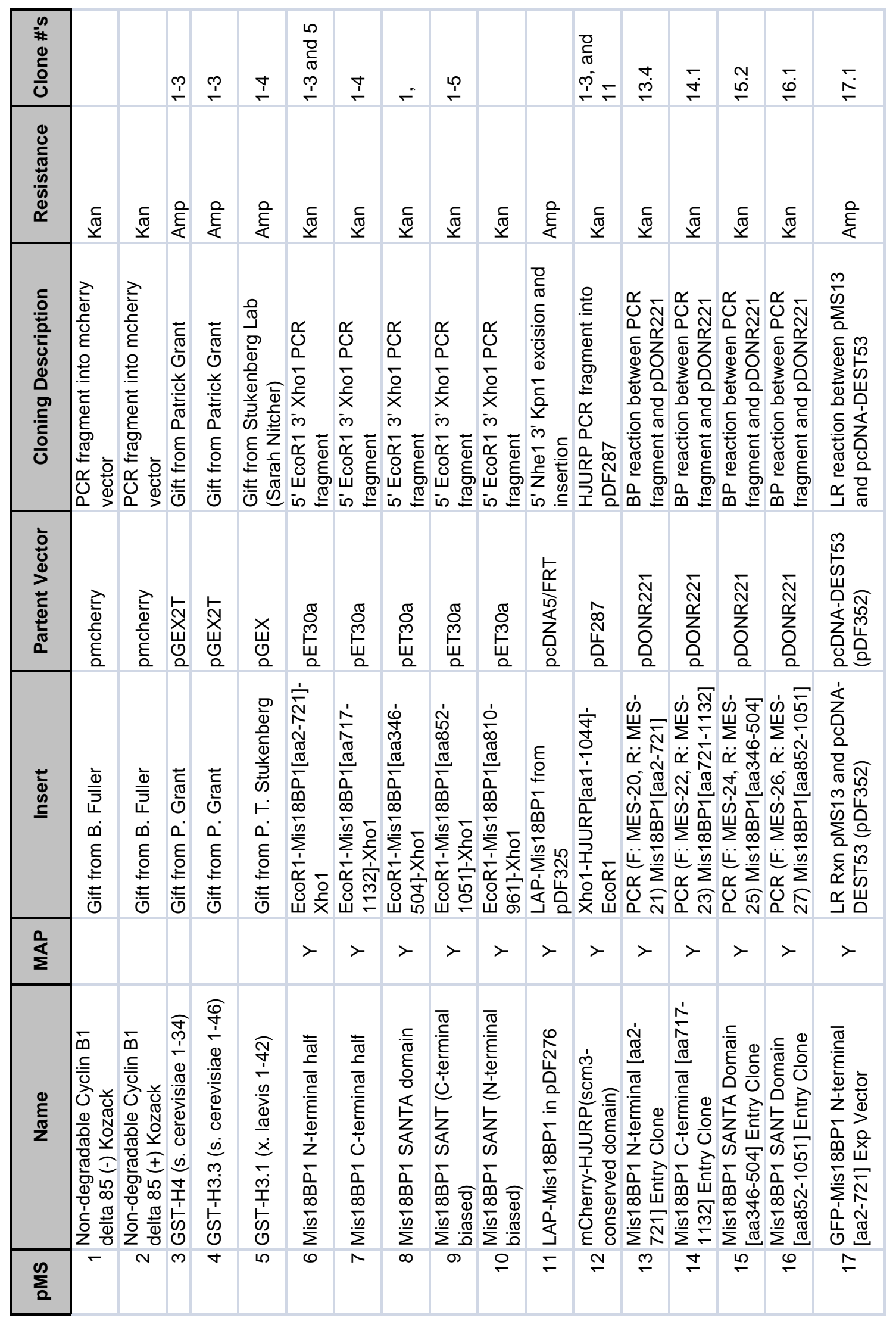




\begin{tabular}{|c|c|c|c|c|c|c|c|c|c|c|c|c|c|c|}
\hline 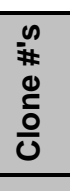 & 孞 & 吕 & $\stackrel{m}{\stackrel{n}{े}}$ & 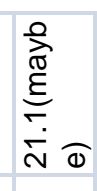 & $\overrightarrow{\mathrm{N}}$ & $\stackrel{\vec{D}}{\sim}$ & $\stackrel{\vec{I}}{\stackrel{I}{\mid}}$ & $\stackrel{\vec{\rho}}{\sim}$ & $\stackrel{-1}{\stackrel{N}{N}}$ & $\stackrel{\vec{\sim}}{\sim}$ & $\stackrel{\infty}{\infty}$ & 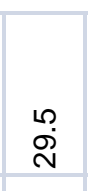 & تُ. & $\vec{m}$ \\
\hline 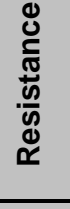 & 亮 & 案 & 突 & 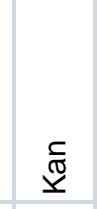 & $\begin{array}{l}\text { ్ָ } \\
\underline{x}\end{array}$ & 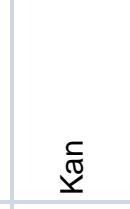 & 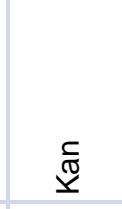 & $\begin{array}{l}\text { ๘్ } \\
\check{\Upsilon}\end{array}$ & 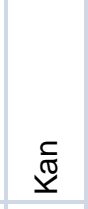 & Ф్ & 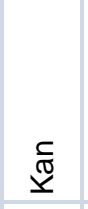 & 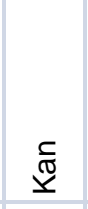 & छे & 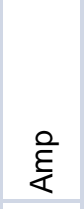 \\
\hline 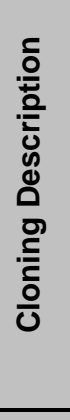 & 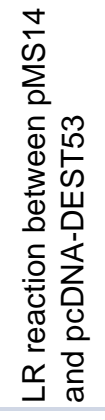 & 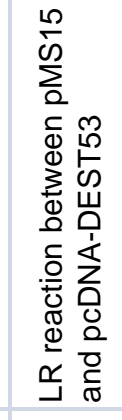 & 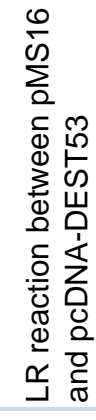 & 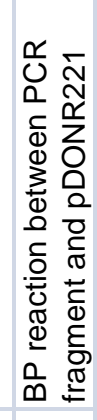 & 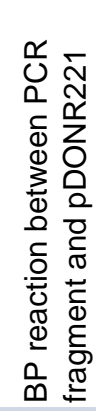 & 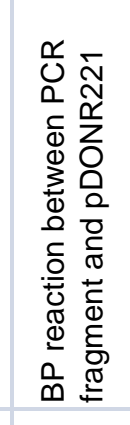 & 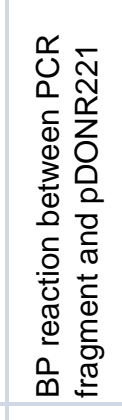 & 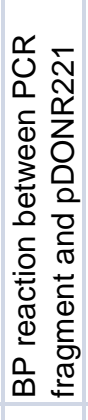 & 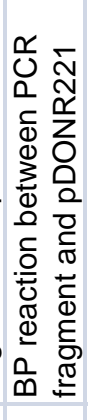 & 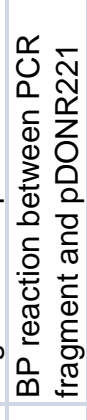 & 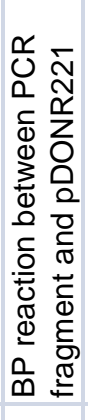 & 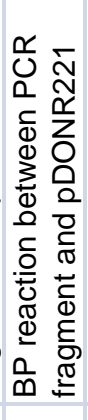 & & \\
\hline 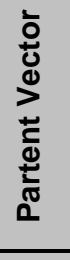 & 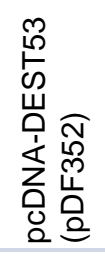 & 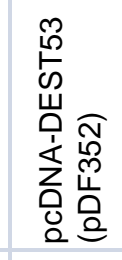 & 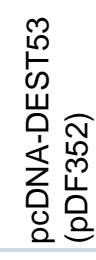 & 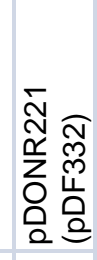 & 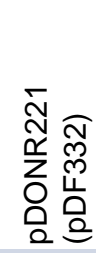 & 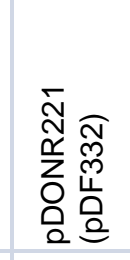 & 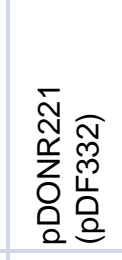 & 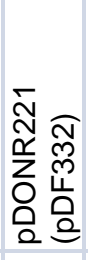 & 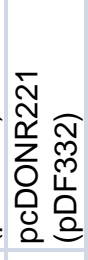 & & $\begin{array}{ll}0 \\
0 \\
0\end{array}$ & 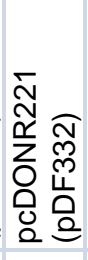 & 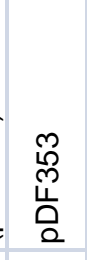 & 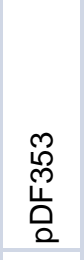 \\
\hline $\begin{array}{l}\text { 嗃 } \\
\stackrel{\omega}{\underline{n}}\end{array}$ & 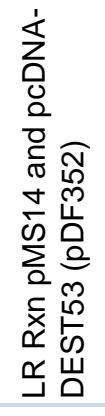 & 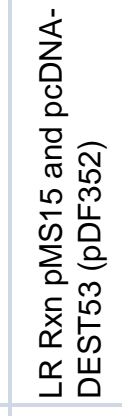 & 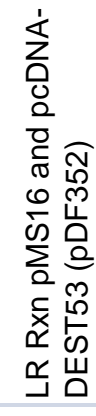 & 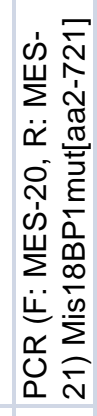 & 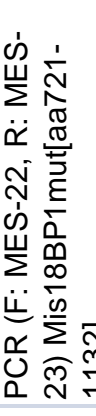 & 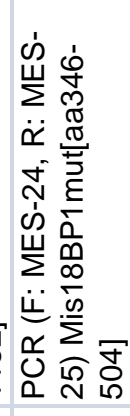 & 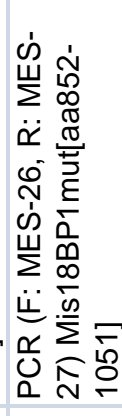 & 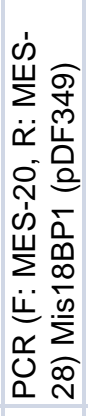 & 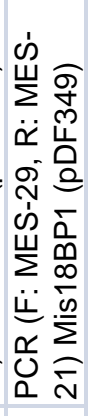 & 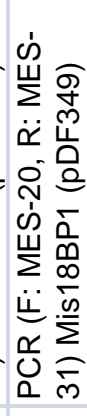 & 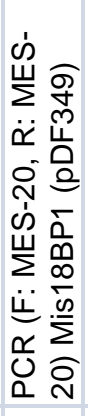 & 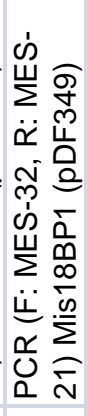 & 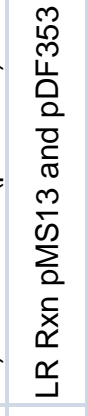 & 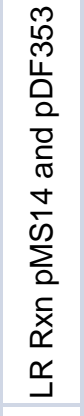 \\
\hline$\frac{Q}{\Sigma}$ & $>$ & $>$ & $\succ$ & $>$ & $>$ & $>$ & $>$ & $>$ & $>$ & $>$ & $>$ & $>$ & $>$ & $>$ \\
\hline$\frac{\sum_{\tilde{\sigma}}^{\frac{\pi}{2}}}{2}$ & 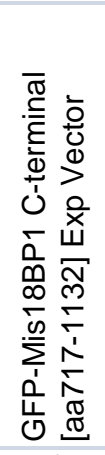 & 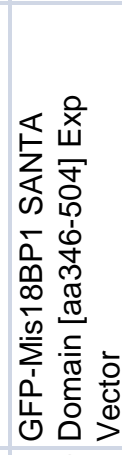 & 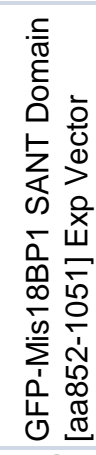 & 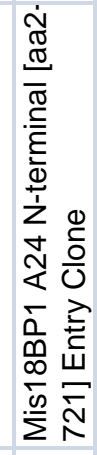 & 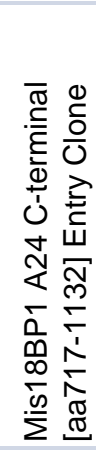 & 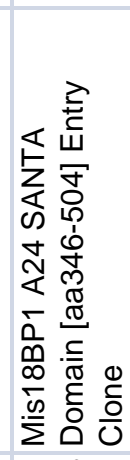 & 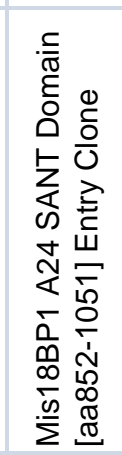 & 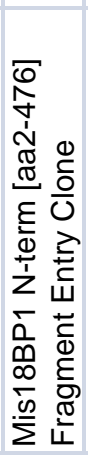 & 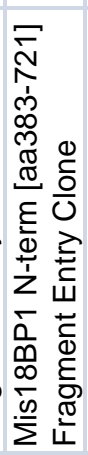 & 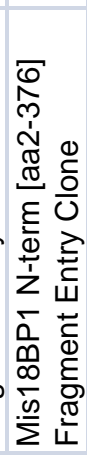 & 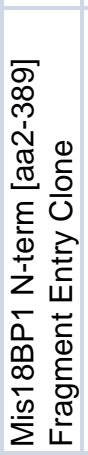 & 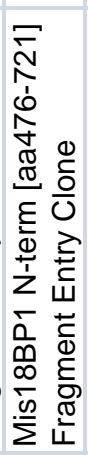 & 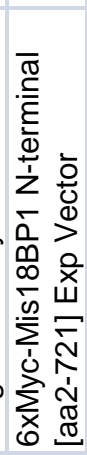 & 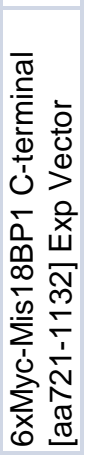 \\
\hline$\sum$ & $\stackrel{\infty}{\rightarrow-1}$ & 9 & 尺े & $\vec{N}$ & $\approx$ & $\mathscr{N}$ & $\stackrel{J}{\sim}$ & $\stackrel{\mathscr{N}}{N}$ & 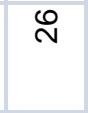 & $\bar{\sim}$ & $\stackrel{\infty}{N}$ & I & 户े & $\vec{m}$ \\
\hline
\end{tabular}




\begin{tabular}{|c|c|c|c|c|c|c|c|c|c|c|c|c|c|c|c|c|c|}
\hline 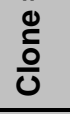 & స్ల & 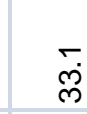 & & $\underset{m}{\stackrel{f}{f}}$ & 岕 & 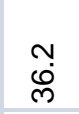 & $\stackrel{\vec{n}}{\stackrel{m}{m}}$ & $\underset{\substack{N \\
\infty}}{\infty}$ & 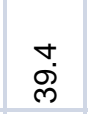 & $\stackrel{m}{q}$ & $\begin{array}{l}\vec{j} \\
\vec{\nabla}\end{array}$ & & & & & & \\
\hline 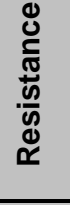 & 灵 & छे & & 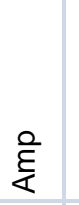 & 享 & 宅 & 灵 & 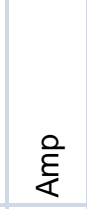 & 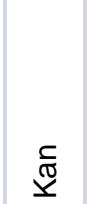 & 產 & 亮 & छे & छे & छे & 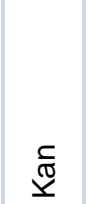 & 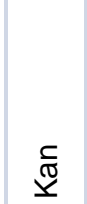 & 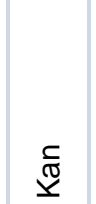 \\
\hline 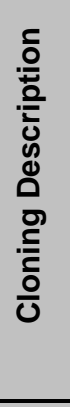 & & & & & & & & & 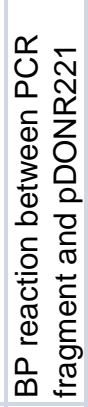 & & & & & & & & \\
\hline 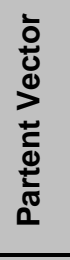 & 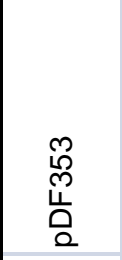 & 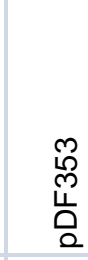 & & 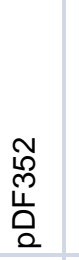 & 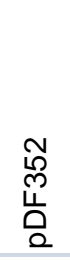 & 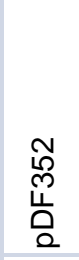 & 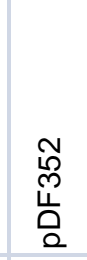 & 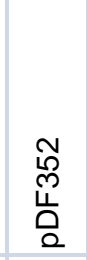 & 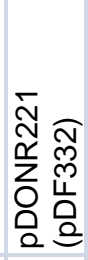 & 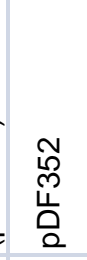 & $\begin{array}{l}\text { P } \\
\stackrel{0}{0} \\
\stackrel{1}{0} \\
\alpha\end{array}$ & 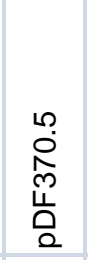 & 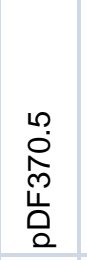 & 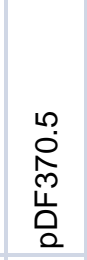 & 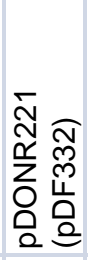 & 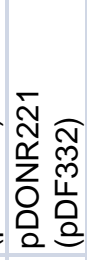 & 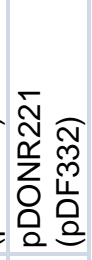 \\
\hline 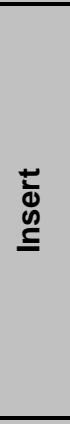 & 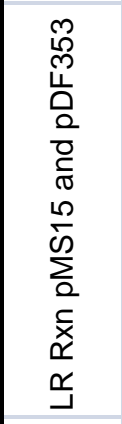 & 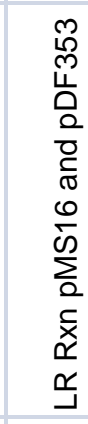 & & 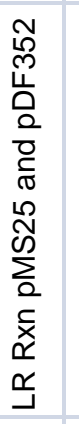 & 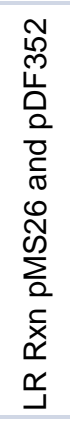 & 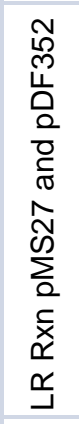 & 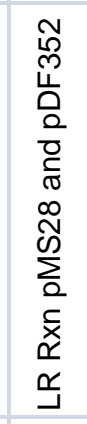 & 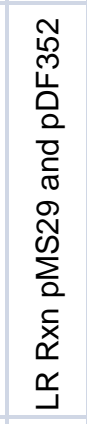 & 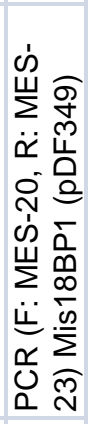 & 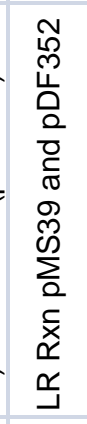 & 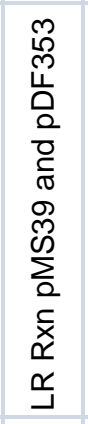 & 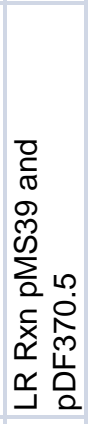 & 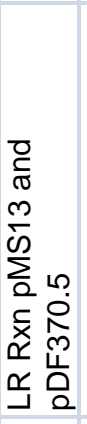 & 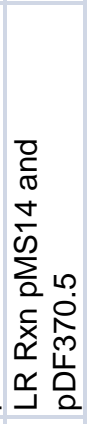 & 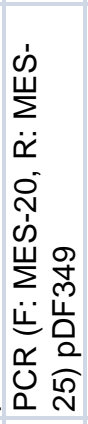 & 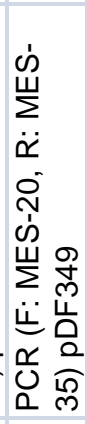 & 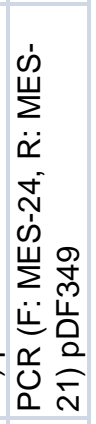 \\
\hline$\frac{Q}{\Sigma}$ & $>$ & $>$ & & $>$ & $>$ & $>$ & $>$ & $>$ & $>$ & $>$ & $>$ & $>$ & $>$ & $>$ & $>$ & $>$ & $>$ \\
\hline 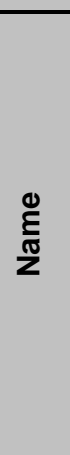 & 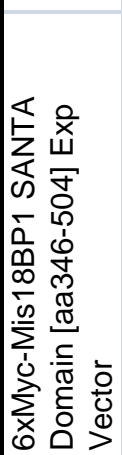 & 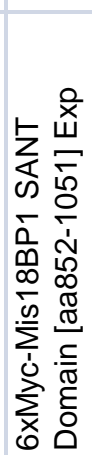 & & 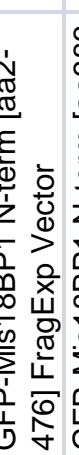 & 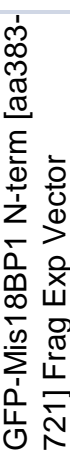 & 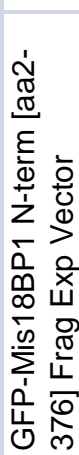 & 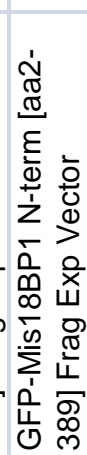 & 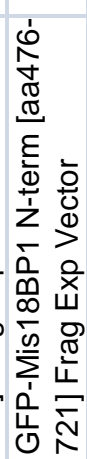 & 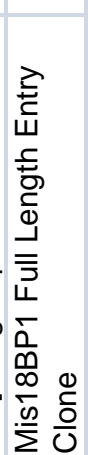 & 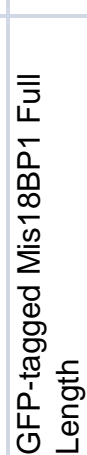 & 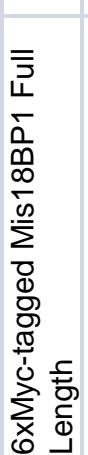 & 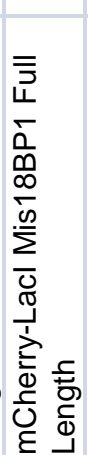 & 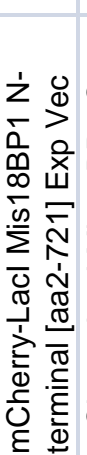 & 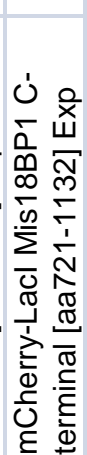 & 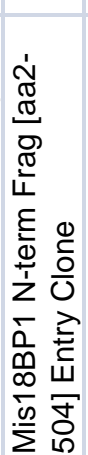 & 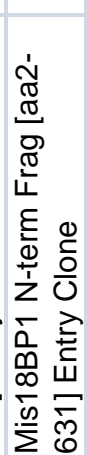 & 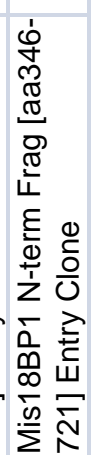 \\
\hline$\sum_{a}^{\infty}$ & $\tilde{N}$ & m & & 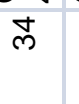 & $\mathscr{m}$ & $\ddot{m}$ & $\hat{m}$ & $\stackrel{\infty}{m}$ & 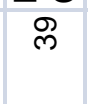 & q & $\vec{\gamma}$ & F & q & 寸 & 过 & o & $\hat{\sigma}$ \\
\hline
\end{tabular}




\begin{tabular}{|c|c|c|c|c|c|c|c|c|c|c|c|c|c|c|c|}
\hline 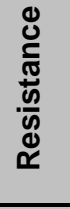 & 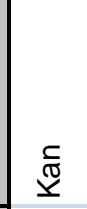 & 胥 & 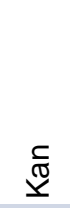 & 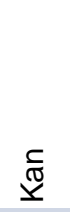 & 育 & 亮 & छे & 亮 & 亮 & हे & 亮 & 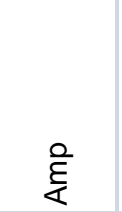 & 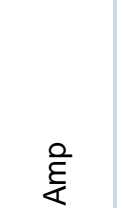 & छे & \\
\hline 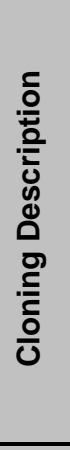 & & & & & & & & & & & & 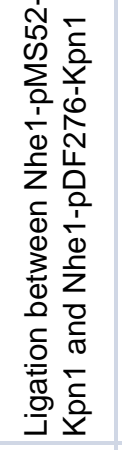 & 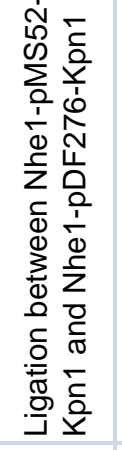 & 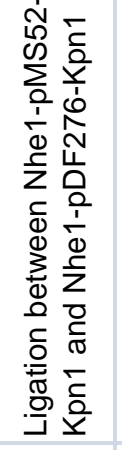 & \\
\hline 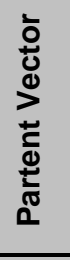 & 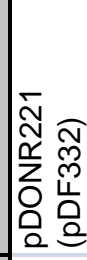 & & & & 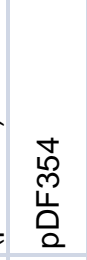 & 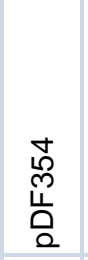 & 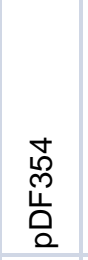 & 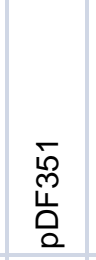 & $\begin{array}{l}\overrightarrow{0} \\
\stackrel{0}{0} \\
\stackrel{1}{0}\end{array}$ & 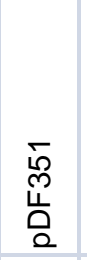 & $\begin{array}{l}\stackrel{ }{0} \\
\stackrel{0}{1} \\
\stackrel{0}{\alpha}\end{array}$ & 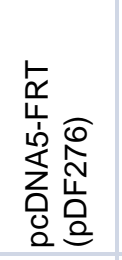 & 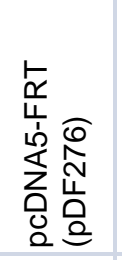 & 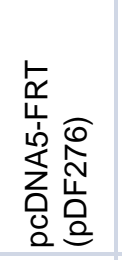 & \\
\hline 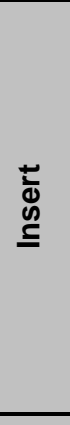 & 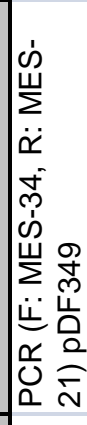 & 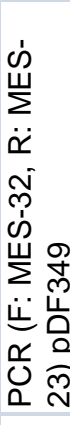 & 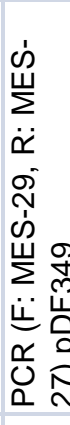 & 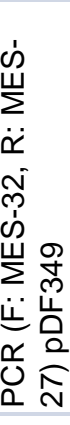 & 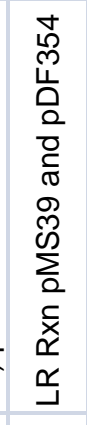 & 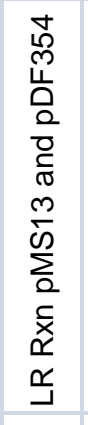 & 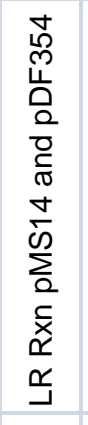 & 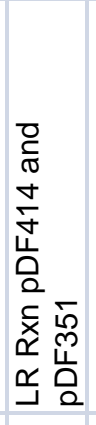 & 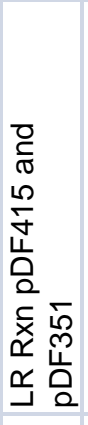 & 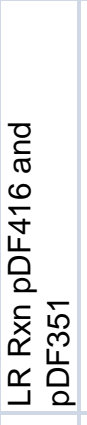 & 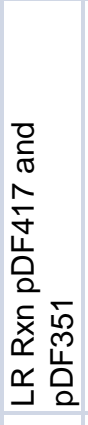 & 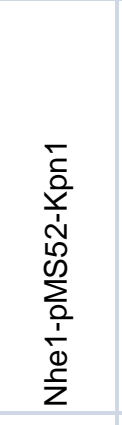 & 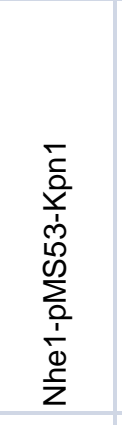 & 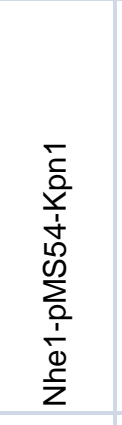 & \\
\hline$\frac{Q}{\Sigma}$ & $>$ & $>$ & $>$ & $>$ & $>$ & $>$ & $>$ & $>$ & $>$ & $>$ & $>$ & $>$ & $>$ & $>$ & $>$ \\
\hline $\begin{array}{l}\stackrel{0}{\tilde{E}} \\
\text { Iू }\end{array}$ & 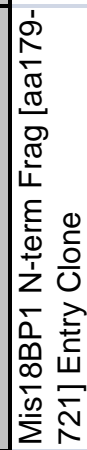 & 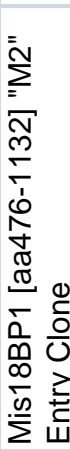 & 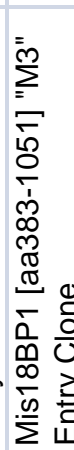 & 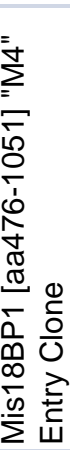 & 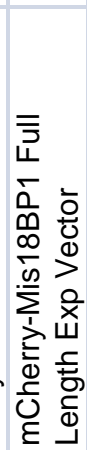 & 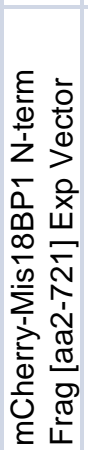 & 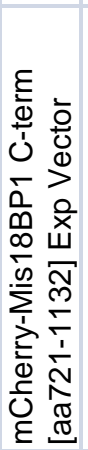 & 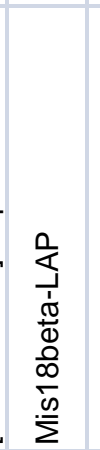 & $\begin{array}{l}\frac{0}{5} \\
\dot{1} \\
\dot{0} \\
\frac{1}{\alpha} \\
\frac{1}{\alpha}\end{array}$ & $\begin{array}{l}\frac{0}{0} \\
\leq \\
0 \\
0 \\
0 \\
\frac{0}{L} \\
\frac{0}{\alpha}\end{array}$ & 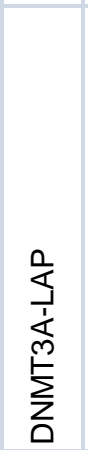 & 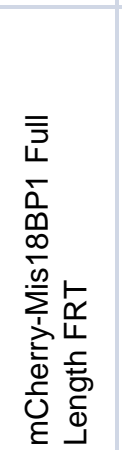 & 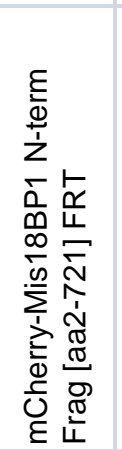 & 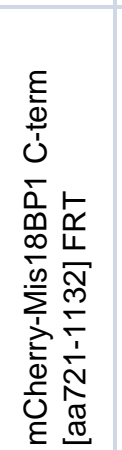 & 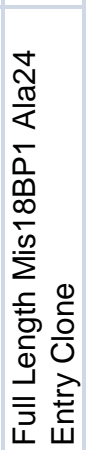 \\
\hline$\sum_{2}^{\infty}$ & $\stackrel{\infty}{\sim}$ & o & 우 & ฝે & กิ & గి & เे & 只 & மீ & in & $\stackrel{\infty}{\llcorner}$ & คి & 8 & $\overrightarrow{6}$ & $\widetilde{ธ}$ \\
\hline
\end{tabular}




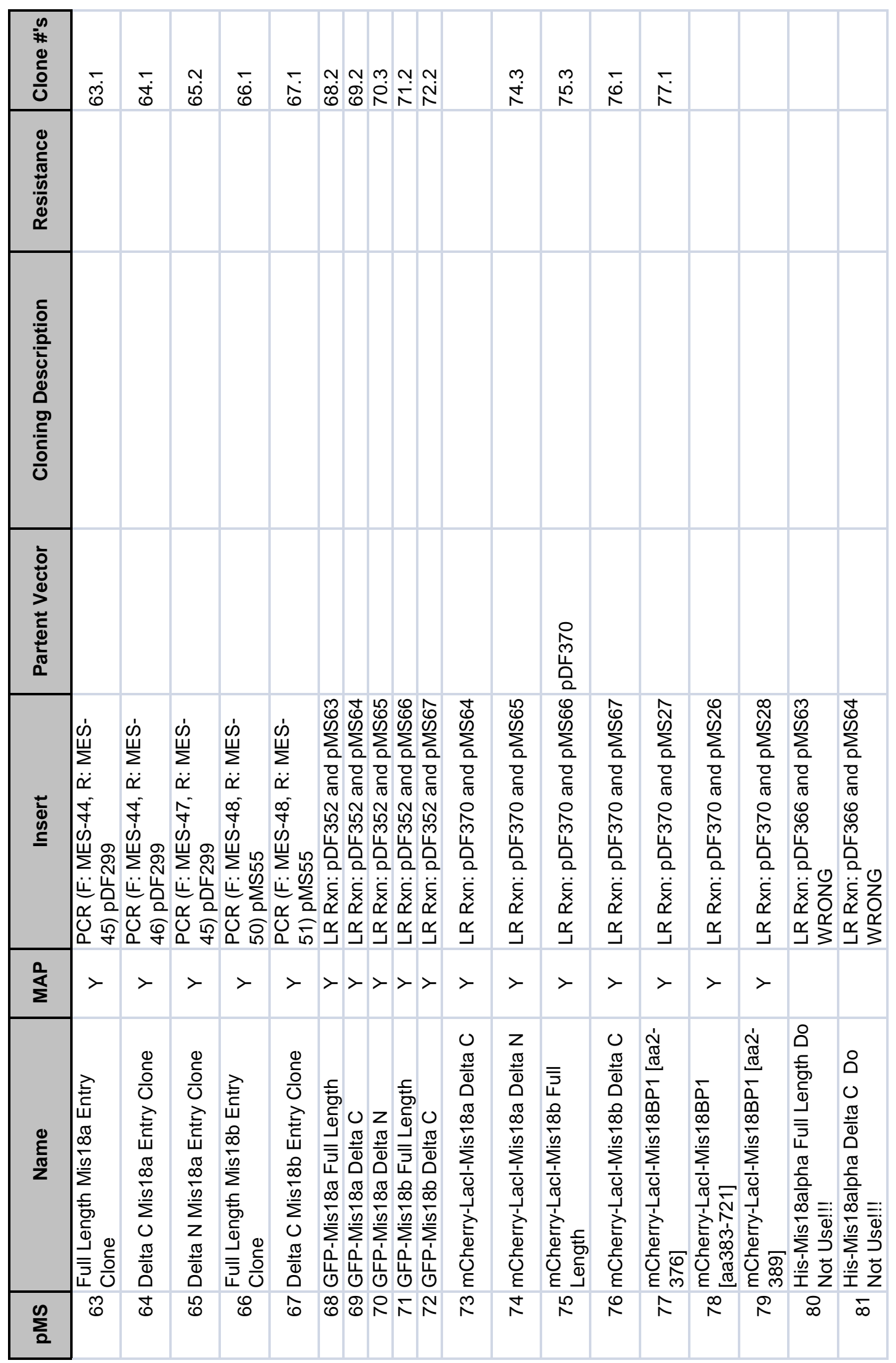




\begin{tabular}{|c|c|c|c|c|c|c|c|c|c|c|c|c|c|c|c|c|}
\hline $\begin{array}{l}0 \\
\# \\
0 \\
\vdots \\
\\
\end{array}$ & & $\underset{\infty}{\infty}$ & $\underset{\infty}{+}$ & 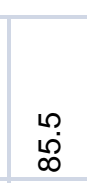 & & & & $\vec{\sigma}$ & & & 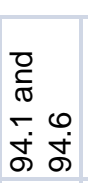 & 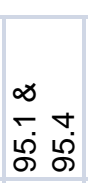 & 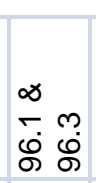 & \begin{tabular}{l}
$\infty$ \\
\multirow{2}{*}{$\tilde{a}$} \\
$\tilde{\sigma}$
\end{tabular} & $\begin{array}{ll}\infty & \\
7 & 1 \\
\infty & 0 \\
\infty & \infty\end{array}$ & $\begin{array}{l}\infty \\
-1 \\
\dot{8} \\
\dot{S} \\
\end{array}$ \\
\hline 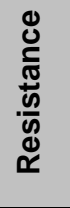 & & $\sum_{<}^{0}$ & $\sum_{i}^{0}$ & & $\sum_{<}^{0} \sum_{i}^{0} \sum_{<}^{0}$ & $\sum_{i}^{0}$ & $\sum_{<}^{0}$ & z & & $\sum_{<}^{0}$ & $\frac{z}{2}$ & $\sum_{i}^{0}$ & $\sum_{i}^{0}$ & $\sum_{<}^{0}$ & $\frac{z}{3}$ & $\sum_{<}^{0}$ \\
\hline 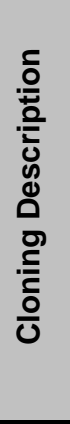 & & & & & & & & & & & 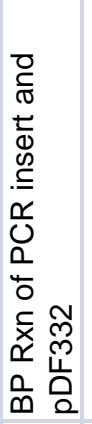 & & & & 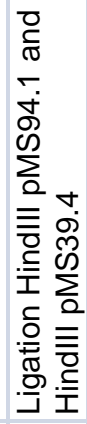 & \\
\hline 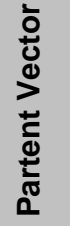 & & & & & & & & & & & & & & & & \\
\hline 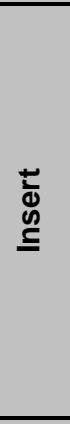 & 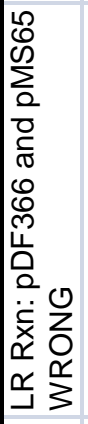 & 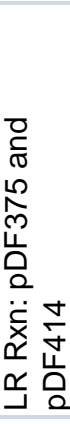 & 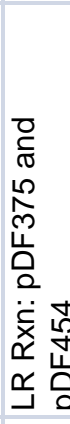 & 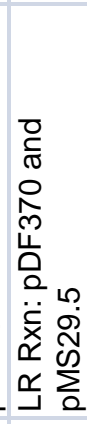 & 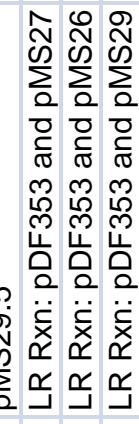 & 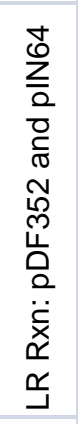 & 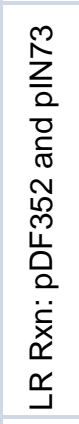 & 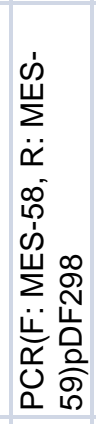 & 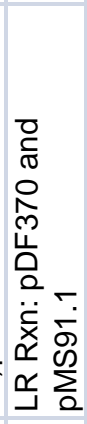 & & 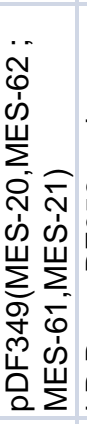 & 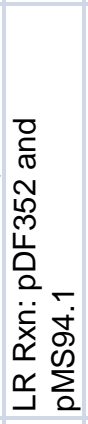 & 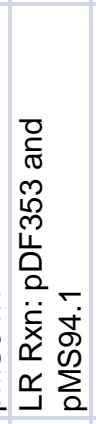 & 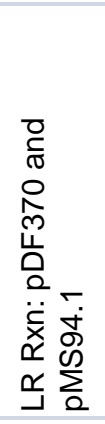 & 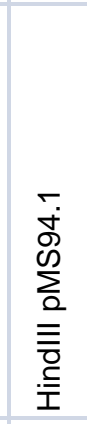 & 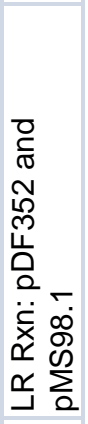 \\
\hline$\frac{0}{\Sigma}$ & & $>$ & $>$ & $>$ & $>>>$ & $>$ & $>$ & $>$ & $>$ & & $>$ & $>$ & $>$ & $>$ & $>$ & $>$ \\
\hline 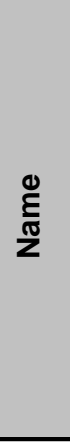 & 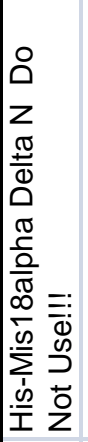 & 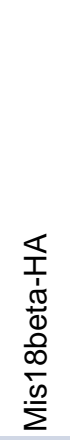 & 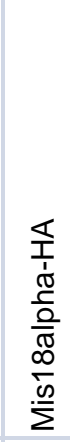 & 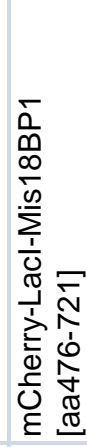 & 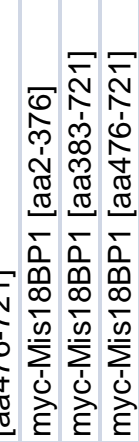 & 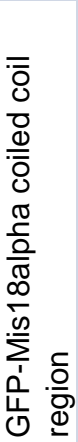 & 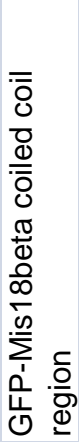 & 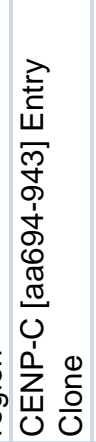 & 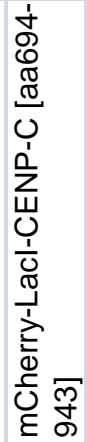 & 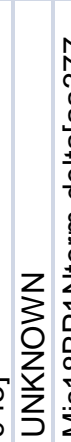 & 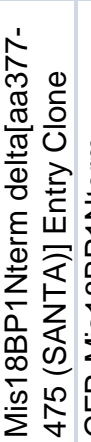 & 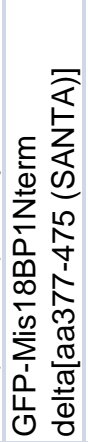 & 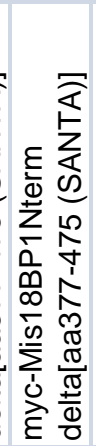 & 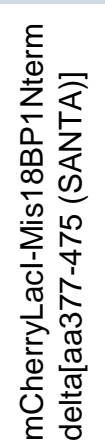 & 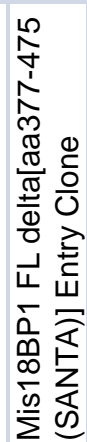 & 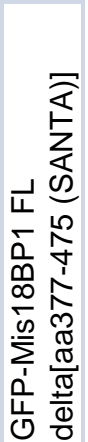 \\
\hline$\sum_{2}^{n}$ & $\tilde{\infty}$ & $\infty$ & 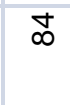 & $\stackrel{\infty}{\infty}$ & 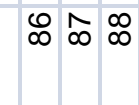 & @ & 8 & बे & $\tilde{\sigma}$ & ஜூ & मे & ஜூ & 8 & ลิ & $\infty$ & 8 \\
\hline
\end{tabular}




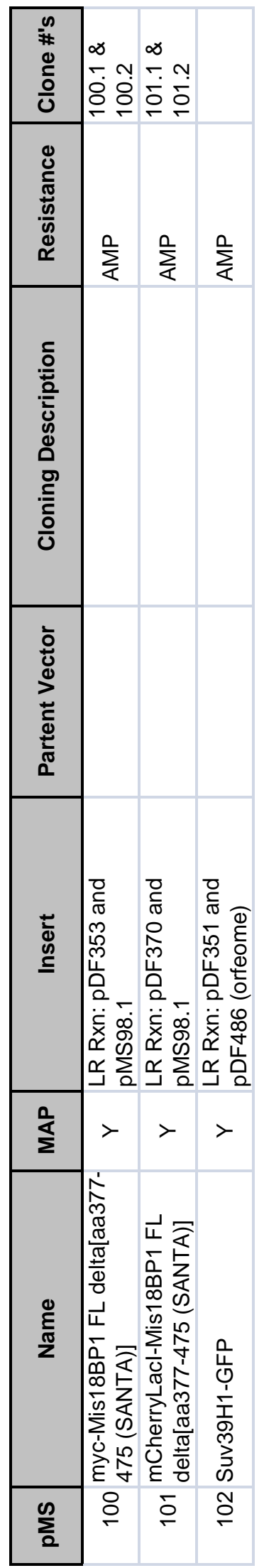

